\title{
A PILOT INVESTIGATION OF MILD HYPOTHERMIA IN NEONATES RECEIVING EXTRACORPOREAL MEMBRANE OXYGENATION (ECMO)
}

Thesis submitted for the degree of

Doctor of Medicine

At the University of Leicester

by

\author{
Marie Horan \\ MBChB (Liverpool) \\ MRCP (UK) \\ $\mathrm{MRCPCH}$
}

February 2007 
UMI Number: U491533

All rights reserved

\section{INFORMATION TO ALL USERS}

The quality of this reproduction is dependent upon the quality of the copy submitted.

In the unlikely event that the author did not send a complete manuscript and there are missing pages, these will be noted. Also, if material had to be removed, a note will indicate the deletion.

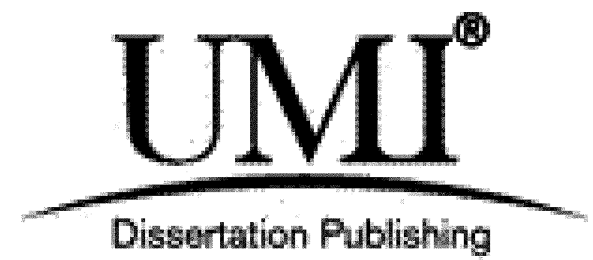

UMI U491533

Published by ProQuest LLC 2013. Copyright in the Dissertation held by the Author.

Microform Edition (c) ProQuest LLC.

All rights reserved. This work is protected against unauthorized copying under Title 17, United States Code.

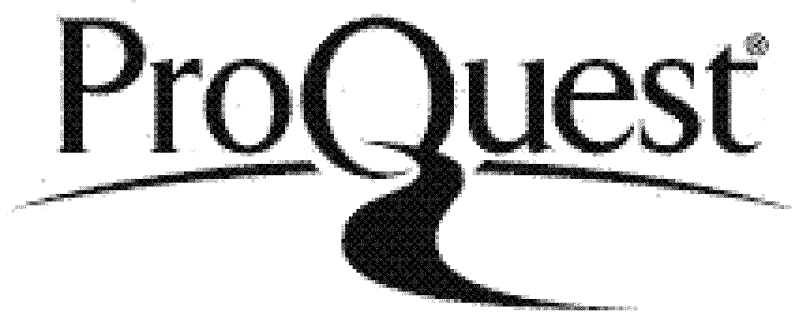

ProQuest LLC

789 East Eisenhower Parkway

P.O. Box 1346

Ann Arbor, MI 48106-1346 
To my parents, without whose vision and dedication this thesis would not have been written 


\section{PREFACE}

The subject of this thesis is the feasibility of applying mild hypothermia during neonatal extracorporeal membrane oxygenation (ECMO). ECMO is an invasive form of life support used in patients with severe but potentially reversible respiratory or cardiac failure.

ECMO is usually considered when conventional treatment has failed, and patients are at very high risk of dying $(80 \%$ to $100 \%)$. Therefore neonates who require ECMO are by definition critically ill and at high risk of hypoxic-ischaemic cerebral injury. We know from the results of the UK ECMO trial that ECMO offers the best chance of survival in such a group of infants. However, the four year follow up data of patients in the neonatal ECMO trial showed that significant numbers of both ECMO and conventionally treated infants had neurological impairment. For ECMO survivors, it is unclear whether this is related to the severity of their initial illness or ECMO itself. Certainly during cannulation for ECMO, there are changes in the cerebral circulation, which may contribute to the risk of later problems. It is plausible, therefore, that protecting the brain in the immediate hours following cannulation for ECMO may improve the neurological outcome in survivors of neonatal ECMO.

From the results of animal data, mild hypothermia appears to be a promising means of neuroprotection following hypoxic-ischaemic cerebral injury. Translating these promising experimental results into clinical practice poses a number of challenges not least the potential adverse effects of mild hypothermia. The documented adverse effects of hypothermia include immune suppression, coagulation dysfunction and cardiac 
arrhythmias. In applying mild hypothermia during ECMO, one must consider the adverse effects not only in the patient but also within the extracorporeal circuit. Patients receiving ECMO are routinely systemically heparinised which might compound the potential increased risk of haemorrhage during cooling. Also, the increased blood viscosity caused by cooling might interfere with the functioning of the membrane oxygenator or cause an embolus in the patient.

It was clear that a definite answer regarding the neuroprotective effect of mild hypothermia would require a randomised controlled trial. It seemed inappropriate to embark on such a trial without first carrying out a study to demonstrate that the approach was feasible.

The aim of this thesis therefore is to investigate the feasibility of using mild hypothermia in neonates receiving ECMO. The infants were whole body cooled by adjustment of the temperature of the water bath in the extracorporeal circuit. During cooling and rewarming, the infants were carefully assessed clinically and biologically. In particular coagulation and immunological markers were measured, the QTc interval monitored continuously using a digital ECG monitor and the amplitude integrated electroencephalogram recorded using a cerebral function monitor. 


\section{TABLE OF CONTENTS}

iii-iv) Preface

Chapter 1: An Overview of Neonatal ECMO: The Need for

Neuroprotection and Mild Hỵpothermia as a Neural Rescue Therapy. P 1-69

Introduction

1.1 Aims of Thesis

1.2 History of ECMO

1.3 Indications for ECMO

1.4 Selection Criteria for ECM0

1.5 The Outcome of Neonatal ECMO

1.6 Why the Need for Neuroprotection?

1.7 The Background for Neuroprotection 25

1.8 Mechanism of Delayed Cell Death 29

1.9 The Concept of the "Therapeutic Window for Neuroprotection" 37

$\begin{array}{ll}1.10 \text { Neural Rescue Therapies } & 37\end{array}$

1.11 Mild Hypothermia as a Neuroprotective Strategy 53

1.12 The Challenges of Applying Mild Hypothermia in a Clinical Setting 57

1.13 Adverse Effects of Mild Hypothermia 63

1.14 The Accuracy of Laboratory Measurements During Cooling 69

\section{Chapter 1: Figures and Tables}

Figure 1 Neonatal Circuit Diagram

Table 1 Contraindications to Neonatal ECMO $\quad 19$

Table 1.1 Overall Outcome of Neonatal ECMO 20

Table 1.2 Neonatal Respiratory Outcome by Diagnosis 20

Chapter 2: Ethics. P 70-72

Issues of Parental Consent

2.1 Independent Data Monitoring Committee

Chapter 3: Methodological Aspects. P 73-88

Design of the Study $\quad 73$

3.1 Methods $\quad 73$

$\begin{array}{ll}3.2 \text { Illustrations of ECMO Circuit } & 77\end{array}$

$\begin{array}{ll}3.3 \text { Study Group } & 80\end{array}$

3.4 Hypothermic Treatment $\quad 81$

3.5 The Heat Exchanger and Water Heater 83

3.6 Measurement of the Core Temperature $\quad 85$

3.7 Monitoring and Blood Sample Collection $\quad 87$ 
Chapter 4: Demographic Data and Core Temperature Control. P 89 -95

$\begin{array}{lr}\text { Demographic Data } & 89\end{array}$

4.1 Core Temperature 90

4.2 Tables and Figures $\quad 91$

Chapter 4: Figures and Tables

Table 1 Demographic Data $\quad 91$

Table 2 Progress on ECMO and Complications $\quad 92$

Figure1 Rectal Temperature of Study Groups 93

Chapter 5: The Effect of Temperature on the QTe Interval. P 96-116

$\begin{array}{ll}\text { Introduction } & 96\end{array}$

5.1 Methods 97

5.2 Problems on Obtaining Continuous QTc Measurement 99

5.3 QT Measurement Using a 24 Hour Digital ECG Holter Recorder 102

$\begin{array}{ll}\text { 5.4 The Digital 24 Hour ECG Holter } & 104\end{array}$

5.5 Twelve lead Electrocardiogram Validation 105

$\begin{array}{ll}\text { 5.6 Statistical Analysis } & 105\end{array}$

$\begin{array}{ll}5.7 \text { Results } & 106\end{array}$

$\begin{array}{ll}5.8 \text { Discussion } & 108\end{array}$

$\begin{array}{ll}5.9 \text { Conclusions } & 111\end{array}$

$\begin{array}{ll}5.10 \text { Figures and Tables } & 113\end{array}$

\section{Chapter 5: Figures and Tables}

Figure 1 Bland Altman Plot Comparing QT Interval Measured by Both 113 Standard 12 Lead ECG and Digital Monitor

$\begin{array}{ll}\text { Table } 1 \text { Demographic Data } & 114\end{array}$

Table 2 Automated QT and QTc Interval During Cooling and Rewarming 115

Figure 2 Scatter Plots for QTc Measured Using a Digital Monitor 116

Chapter 6: The Effects of Mild Hypothermia on Coagulation P118 - 134

$\begin{array}{ll}\text { Introduction } & 118\end{array}$

6.1 Challenges of Measuring Coagulation During Hypothermia 119

$\begin{array}{ll}\text { 6.2 Molecular Markers of Coagulation } & 121\end{array}$

6.3 The Activated Clotting Time 122

$\begin{array}{ll}6.4 \text { The Hemochron ACT Monitor } & 125\end{array}$ 
$\begin{array}{ll}6.5 \text { Methods } & 126\end{array}$

$\begin{array}{lr}6.6 \text { Results } & 128\end{array}$

$\begin{array}{lr}6.7 \text { Discussion } & 129\end{array}$

$\begin{array}{lr}6.8 \text { Conclusions } & 130\end{array}$

6.9 Figures and Tables 132

Chapter 6: Figures and Tables

Figure 1 Heparin Requirements for Study Groups 132

Figure 2 Heparin Requirements for Group $5 \quad 132$

$\begin{array}{lr}\text { Figure } 3 \text { Molecular Markers of Coagulation } & 133\end{array}$

Figure 4 Coagulation Parameters for Study Groups 134

Chapter 7: Cardiovascular Changes During Mild Hypothermia and Rewarming P 136 - 145

$\begin{array}{ll}\text { Introduction } & 136\end{array}$

$\begin{array}{ll}7.1 \text { Methods } & 139\end{array}$

$\begin{array}{ll}7.2 \text { Statistical Analysis } & 140\end{array}$

$\begin{array}{ll}7.3 \text { Results } & 140\end{array}$

$\begin{array}{ll}7.4 \text { Discussion } & 141\end{array}$

$\begin{array}{ll}7.5 \text { Conclusions } & 142\end{array}$

7.6 Figures and Tables 143

\section{Chapter 7: Figures and Tables}

Table 1 Cardiovascular Changes During Cooling and Rewarming 143 Table 2 Rectal Temperature and Cardiovascular Data During Mild 144 Hypothermia

$\begin{array}{ll}\text { Figure } 1 \text { Persistent Tachycardia During Cooling } & 145\end{array}$

Chapter 8: Immunology and Mild Hypothermia P 146 - 153

$\begin{array}{lr}\text { Introduction } & 146\end{array}$

8.1 Cytokines IL-6 and IL-8 147

$\begin{array}{ll}\text { 8.2 Complement C3a } & 148\end{array}$

$\begin{array}{lr}8.3 \text { Methods } & 149\end{array}$

$\begin{array}{lr}8.4 \text { Results } & 150\end{array}$

$\begin{array}{ll}\text { 8.5 Discussion } & 151\end{array}$

$\begin{array}{ll}\text { 8.6 Conclusions } & 151\end{array}$

8.7 Figures and Tables 152 


\section{Chapter 8: Figures and Tables}

Figure 1 Cytokines IL-6 and IL-8 for Study Groups 152

Figure 2 Complement C3a for Study Groups 153

Figure 3 C-Reactive protein for Study Groups 153

Chapter 9: The Influence of Temperature on the Amplitude Integrated Electroencephalogram During Neonatal ECMO P 154 - 176

Introduction 154

9.1 Methods 155

9.2 Statistical Analysis 163

9.3 Results 163

9.4 Discussion 166

9.5 Conclusions 170

9.6 Figures and Tables 171

\section{Chapter 9: Figures and Tables}

Figure 1 Measurement of aEEG 160

Figure 2 Classification of aEEG Traces 161

Figure 3 Measurement of Effect of Mild Hypothermia 162

Table 1 Demographic Data 171

Figure 4 Median Core Temperature for Study Groups 172

Table 2 Summary of aEEG Traces 173

Table 3 Summary of aEEG Voltage During the Last 6 hours of Cooling 174 and the first 6 Hours of Rewarming

Table 4 Paired Samples Test of aEEG Voltage during the Last 6 hours of 175

Cooling and the first 6 Hours of Rewarming

Figure 5 Changes in aEEG with Acute Neurological Complications 176

Chapter 10: Summary and Conclusions P 177 - 181

10.1 Introduction 177

10.2 Summary 177

10.3 Conclusions 179

10.4 Future Direction of Research 179

10.5 Contribution to Clinical Knowledge of Hypothermia 181

Acknowledgements 182

Appendix 1: Publications Arising from the Thesis 183

Statistical Methods $\quad 184$

$\begin{array}{ll}\text { Bibliography } & 185\end{array}$ 


\section{CHAPTER 1}

\section{AN OVERVIEW OF NEONATAL ECMO : THE NEED FOR \\ NEUROPROTECTION AND MILD HYPOTHERMIA AS A NEURAL \\ RESCUE THERAPY}

\section{INTRODUCTION}

Extracorporeal membrane oxygenation (ECMO) is an invasive form of life support used in intensive care for neonatal, paediatric and adult patients with severe cardiac and/or respiratory failure. The technique uses a modified cardiac bypass circuit and involves insertion of a wide bore cannula into a central vein (usually the right internal jugular vein) and the right common carotid artery (veno-arterial ECMO) or a double lumen venous cannula (veno-venous ECMO). Blood is drained by gravity from the venous cannula and propelled forward around the circuit by a roller pump. Following oxygenation by a silicone membrane oxygenator, the blood is warmed by a countercurrent heat exchanger before re-entering the patient via the return cannula. (see figure1)

Infants are referred for ECMO because of hypoxia despite maximum conventional therapy. Hence neonates receiving extracorporeal membrane oxygenation (ECMO) represent a group at high risk of brain injury.

The UK ECMO trial ${ }^{1}$ showed the benefit of ECMO compared to conventional treatment in such critically ill neonates in terms of both survival and neurodevelopmental outcome. However, the four year follow up data of infants in the 
study showed $50 \%$ of ECMO survivors and $63 \%$ of conventionally treated survivors had a degree of neurological impairment. ${ }^{2}$ For ECMO survivors, it is unclear whether this is related to the severity of their initial illness or ECMO itself. Certainly during cannulation there are changes in the cerebral circulation, which may contribute to the risk of later problems. It is plausible, therefore, that protecting the brain in the immediate hours following cannulation for ECMO may improve the neurological outcome in survivors of neonatal ECMO.

Mild hypothermia appears to be a promising means of neuroprotection. Evidence from studies in animals have shown that a reduction in the body temperature by $3^{\circ} \mathrm{C}$ or $4^{0} \mathrm{C}$ after an hypoxic ischaemic insult preserves cerebral energy metabolism, reduces cytotoxic oedema, and improves histological and behavioural outcome..$^{3-6}$ Translating these promising experimental results into clinical practice poses a number of challenges not least the concern of the potential adverse effects of cooling. Documented complications of mild hypothermia include cardiac effects ${ }^{7-9}$, immune suppression $^{10-11}$ and coagulation dysfunction. ${ }^{12-15}$ With particular regard to applying mild hypothermia during ECMO, consideration must also be given to theoretical effects of cooling both on the patient and the extracorporeal circuit.

Patients receiving ECMO are routinely systemically heparinised which might compound the potential increased risk of haemorrhage during cooling. Also, the increased blood viscosity caused by cooling might interfere with the functioning of the membrane oxygenator or cause an embolus in the patient. It was clear that a definite answer regarding the neuroprotective effect of mild hypothermia would require a randomised controlled trial. It seemed inappropriate to 
embark on such a trial without first carrying out a study to demonstrate that the approach was safe. The aim of this pilot study was to investigate the feasibility of using mild hypothermia in neonates receiving ECMO.

In this chapter the aims of the thesis are stated. I explore the history, indications for and neurological outcomes of neonatal ECMO. I will discuss the aetiology of cerebral injury in ECMO treated neonates. A literature review of the evidence for neuroprotective strategies (in particular mild hypothermia) is presented and the potential adverse effects of cooling discussed. Finally, the accuracy of laboratory measurements during cooling is discussed. 
Figure 1 Schematic Drawing of a Neonatal ECMO Circuit

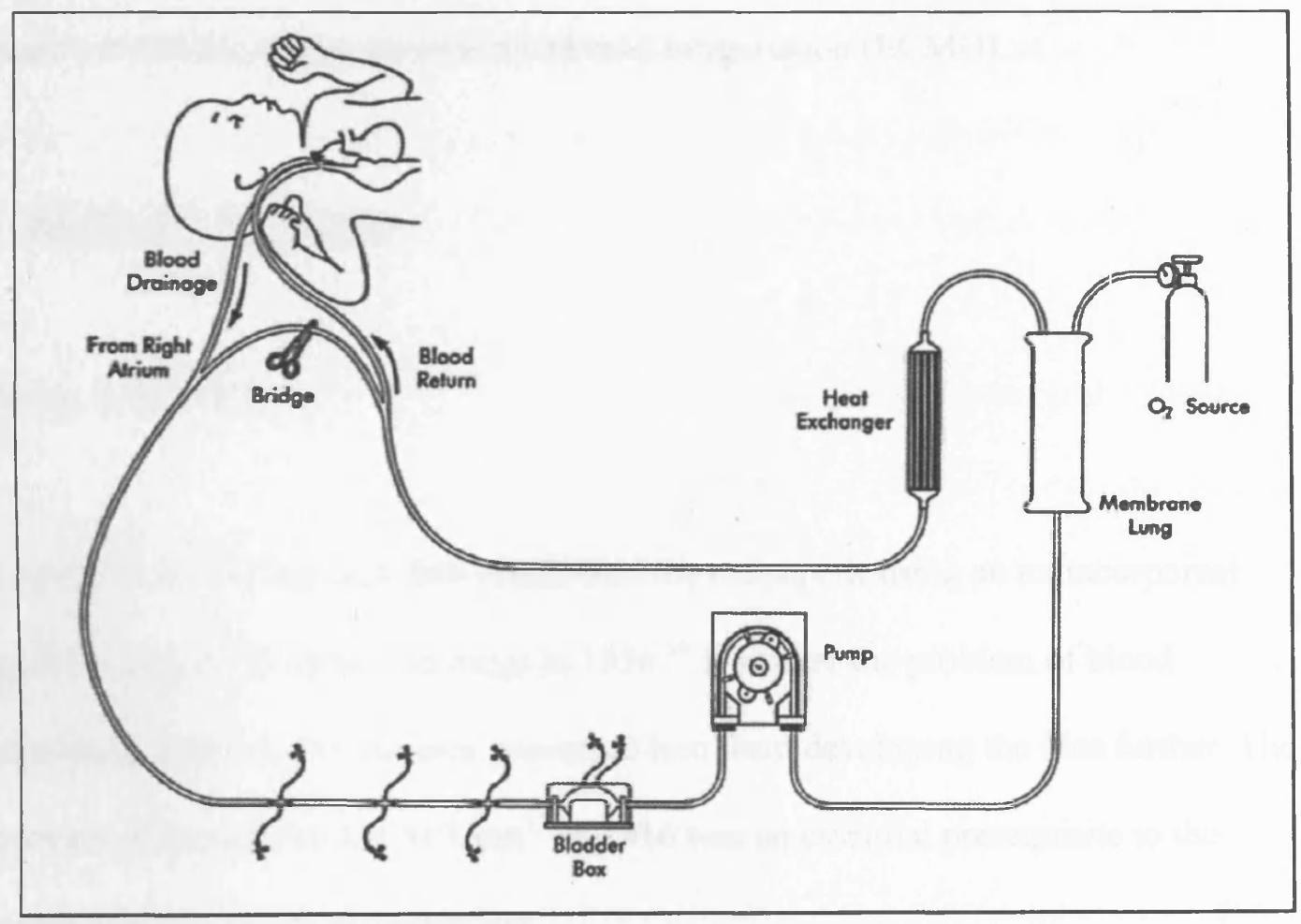




\subsection{AIMS OF THE THESIS}

The aim of the thesis is to investigate the feasibility of applying mild hypothermia to neonates receiving extracorporeal membrane oxygenation (ECMO).

\subsection{HISTORY OF ECMO}

\section{History of ECMO}

Benjamin Ward Richardson first considered the concept of using an extracorporeal circuit to support the heart and lungs in $1856 .{ }^{16}$ However the problem of blood coagulating within a few minutes prevented him from developing the idea further. The discovery of heparin by Jay $\mathrm{M}^{\mathrm{c}} \mathrm{Lean}^{17}$ in 1916 was an essential prerequisite to the success of extracorporeal circulation.

Open-heart surgery is widely regarded as one of the most important developments in twentieth century medicine. The era began in 1952 when Lewis carried out the first successful closure of an atrial septal defect under direct vision. ${ }^{18}$ Since this milestone, there has been a rapid evolution in the methods of diagnosis and correction of cardiac lesions. The cornerstone to the expansion in this field has been extracorporeal circulation. This technique has allowed surgeons to empty the heart of blood, stop its beat as necessary, open any desired chamber and operate safely in an unhurried manner.

The idea of coupling extracorporeal circulation and oxygenation originated with Gibbon. In 1932, following the tragic death of a gravid woman patient with a pulmonary embolus, he began work on a pump oxygenator. ${ }^{19}$ Gibbon was the first to 
establish the feasibility of artificially supported circulation during temporary occlusion of the pulmonary artery. ${ }^{20}$ Over the next twenty years, Gibbon refined the technique of cardiopulmonary bypass. The first successful application of a heart-lung machine came in 1953 when Gibbon used a pump oxygenator to close an atrial secundum defect. ${ }^{21}$ However with five failures out of the six patients he operated on using the heart-lung machine Gibbon became discouraged and abandoned open-heart surgery.

Various groups continued to investigate the clinical use of cardiopulmonary bypass with a lack of reported success. Therefore, other modes of circulatory support during cardiac surgery were developed including cross-circulation. The principle of crosscirculation was to use venous and arterial cannulae to connect the donor patient's circulation to the recipient patient undergoing open heart surgery. A single motor pump was used to control the reciprocal exchange between the donor and the patient. In 1954, cross-circulation was successfully used for the closure of a ventricular septal defect in a 12-month-old infant. ${ }^{22}$ From March 1954 to July 1955, 28 out of 45 patients survived this technique. ${ }^{23}$ In 1955 DeWall and Lillehei reported the successful use of a simple disposable bubble oxygenator used during the closure of ventricular septal defects in seven patients. ${ }^{24}$ Also in 1955, Kirklin and Wood described their experimental results using a Gibbontype pump oxygenator. ${ }^{25-26}$

However, these heart-lung machines could only give life support for short periods of time with lethal consequences when used for more than a few hours. The toxic manifestations included diffuse capillary leakage, poor perfusion, acidosis, heamolysis, thrombocytopenia, coagulopathy and progressive organ failure. This toxicity was traced to the artificial lung and specifically to the direct interface between 
blood and gas. Gross foaming, macro- and microbubbles and heamolysis occurred if the procedure was continued long enough. Prolonged life support using ECMO was not possible until major improvements in oxygenator design had occurred. Progress came in 1957, when Kammermeyer synthesised silicone rubber. ${ }^{27}$ This material was strong enough to withstand considerable hydrostatic pressure yet permeable to gas exchange. The membrane oxygenators developed by Kolobow, ${ }^{28}$ Kloff, ${ }^{29}$ Bramson $^{30}$ and others were the first that separated the blood gas phases in the device with a silicone membrane. This resulted in less red cell haemolysis and manageable platelet consumption. In the late 1960 's following the development of the membrane artificial lungs, problems such as vascular access, bleeding, heparin management and air embolism were gradually resolved using animal experiments. ${ }^{31-33}$ In $1972 \mathrm{Hill}_{\mathrm{e}} \mathrm{al}^{34}$ reported the first successful use of ECMO in an adult patient with severe respiratory failure. This patient was a 24 four-year-old male who had sustained transection of the thoracic aorta and multiple fractures following a motor cycle accident. His respiratory function deteriorated and he was maintained on venoarterial extracorporeal life support for 75 hours. Reports of several other successful cases soon followed. ${ }^{35-36}$

In 1975, the American National Institute of Health sponsored a multicentre randomised trial of extracorporeal membrane oxygenation in adult ECMO. ${ }^{37}$ The survival in both the control and the ECMO groups was less than 10 and the study was stopped early with only 92 out of the planned 300 patients having entered the study. As a result of this study, clinical research on ECMO in adult patients in the USA essentially stopped in 1979.

Interest was maintained in the use of ECMO for neonates with respiratory failure. In 1976, Bartlett et $\mathrm{al}^{38}$ reported the first successful neonatal ECMO patient. This was 
followed by other successful cases. ${ }^{39-40}$ In 1982, Bartlett at al published a series of 45 cases where ECMO was used for newborn respiratory failure, which gave encouraging results. ${ }^{41}$ This experience was followed by a prospective controlled randomised trial using the "play the winner" technique. ${ }^{42}$ Twelve patients were recruited into the study. Although producing a statistically significant result the study was heavily criticised, as there was only one control patient, who died. However, the 11 neonates treated with ECMO survived.

By 1986, there were eighteen ECMO centres in the USA. O'Rourke et $\mathrm{al}^{43}$ in 1989 attempted a further prospective randomised study of ECMO in neonates with persistent pulmonary hypertension of the newborn. The results were statistically significant but again, the study design was controversial in that only four deaths were allowed in either arm of the study groups. Also, the control group were not aware that they were in a study which added to the contention surrounding the results of the study.

By the late 1980's neonatal ECMO was standard practice in North America however, there was some resistance to the concept of ECMO from neonatologists in the UK. Before ECMO was formally developed in the UK, a fully randomised control trial was organised. ${ }^{1}$ The results of the UK collaborative ECMO trial showed benefit for ECMO in all patient groups studied. ${ }^{1,44}$ The study also recommended that ECMO should be delivered in a small number of specialised centres with appropriately trained staff.

Despite dwindling interest in adult ECMO, Gattinoni in Milan continued with clinical extracorporeal life support research for adult respiratory failure. He developed the use of veno-venous access to achieve carbon dioxide removal and eliminate the need for high-pressure ventilation and high fractional inspired oxygen. In 1986, Gattinoni 
reported 52\% survival in adult patients using venovenous ECMO. ${ }^{45}$ These results were corroborated by a number of other European centres. ${ }^{46-47}$

\section{Progress to the present day}

The Extracorporeal Life Organisation (ELSO) was established in the USA during the 1980 's as a means of exchanging information on ECMO and producing standardised treatment protocols. ELSO is a consortium of health care professionals who apply and study extracorporeal support of cardiac and pulmonary failure. The development and maintenance of a data registry (ELSO register) is one of the central activities of the Life Support Organisation. The registry was established in 1976 and currently approximately 120 member institutions of the Extracorporeal Life Support Organisation (ELSO) contribute to the ELSO register. The use and refinement of techniques of ECMO have continued to expand and progress through the 1990's to the present day. ${ }^{48}$

\section{Alternative treatment modalities for neonatal pulmonary hypertension}

The development of other treatment modalities such as nitric oxide to treat pulmonary hypertension has expanded the options for conventional therapy in critically ill neonates.

In vivo, nitric oxide is generated enzymatically in the vascular endothelium by one of several nitric oxide synthases from the precursor L-arginine. ${ }^{49}$ Nitric oxide then activates guanylate cyclase by binding to its heme-component, leading to the production of cyclic guanosine 3', 5-monophosphate (GMP). ${ }^{50}$ It is thought that 
cyclic GMP relaxes vascular smooth muscle through the phosphorylation of potassium channels which secondarily inhibits the activation -induced elevation of the cytosolic calcium concentration. This leads to a reduction in the force of contraction of vascular smooth muscle. Nitric oxide is thought to be a major regulator of vascular tone and plays an important role in the regulation of cerebral and coronary blood flow and also participates in vascular haemostasis through its inhibition of platelet aggregation and adhesion.

Studies in animal models showed that inhaled nitric oxide sustained selective pulmonary vasodilatation and at doses of $40-80$ ppm was able to reverse pulmonary hypertension induced by hypoxia. ${ }^{51-53}$ The first reported studies in near -term neonates with pulmonary hypertension by Roberts et $\mathrm{al}^{54}$ and Kinsella et al ${ }^{55}$ showed improvement in oxygenation following administration of inhaled nitric oxide.

Subsequently, the NINOS study showed that very few infants who did not respond to 20 parts per million ( $\mathrm{ppm}$ ) of inhaled nitric oxide responded at higher doses up to $80 \mathrm{ppm}^{56}$

In a systematic Cochrane review of inhaled nitric oxide for respiratory failure for term or near term infants, Finer et $\mathrm{al}^{57}$ found a reduction in the need for ECMO. However, results from the NINOS study, ${ }^{58}$ which separately evaluated patients with congenital diaphragmatic hernia, showed that inhaled nitric oxide did not reduce the need for ECMO or death. Indeed there was a suggestion that the outcome was slightly worsened. One problem related to the "success" of inhaled nitric oxide is that some infants have a slight response to nitric oxide and avoid ECMO but remain ventilated for longer and risk complications such as bronchopulmonary dysplasia. Similarly, infants may possibly be referred later for ECMO by which time they may be out of the accepted treatment criteria which includes less than 10 days ventilation. Also, the 
long term neurodevelopmental and pulmonary function of neonates surviving following inhaled nitric oxide are not known. However, from the results of the data for infants enrolled in the NINOS trial, inhaled nitric oxide was not associated with an increase in neurodevelopment, behavioural or medical abnormalities at age 2 years. ${ }^{59}$ More recently studies of phosphodiesterase -5 inhibitors, in particular sildenafil, have been shown to be specific pulmonary vasodilators. ${ }^{60}$ Phosphdiesterase- 5 inhibitors act by preventing the breakdown of cyclic GMP and may act synergistically with nitric oxide which, as previously mentioned acts by increasing cyclic GMP. A report in a neonatal animal model showed that intravenous sildenafil was at least as effective as inhaled nitric oxide and also increased the cardiac output by $30 \% .^{61}$ In a study in adult patients with severe pulmonary hypertension, Michelakis et $\mathrm{al}^{62}$ also demonstrated that oral sildenafil was as effective as inhaled nitric oxide in reducing pulmonary hypertension. Similar to the findings of Shekerdemian et al, ${ }^{61}$ sildenafil increased the cardiac output. The use of phosphodiesterase- 5 inhibitors for the treatment of neonatal pulmonary hypertension is still in the initial stages of investigation. However, it may prove to be an alternative to inhaled nitric oxide or a useful adjunct. It is unclear whether these newer therapies will further reduce the need for ECMO.

\subsection{INDICATIONS FOR ECMO}

In theory, ECMO can be offered to any patient with potentially reversible pulmonary, cardiac or cardiopulmonary failure. In practice, one has to define a group of patients who are sick enough to justify extracorporeal life support, whilst excluding those 
whose outcome is inevitably hopeless. In general the principles for selecting a potential ECMO candidate are the same for any age group:

1. Does the patient have a potentially reversible pulmonary, cardiac or cardiopulmonary condition?

2. Are the neurological status and function of other organs consistent with a reasonable outcome?

3. Is even limited heparinisation contraindicated?

4. Is there anything to be gained by further pursuit of conventional treatment?

\section{Indications for Neonatal ECMO}

The indications for neonatal ECMO have been relatively standardised (see table 1). The selection criteria are designed to define infants sick enough to require invasive life support, without expecting unacceptable morbidity. ${ }^{63}$ In practice, ECMO is therefore reserved for those patients in whom there remains a possibility of satisfactory outcome despite a failure to respond to maximal conventional therapy. ${ }^{64}$

Each neonate referred as a potential ECMO candidate will therefore be assessed using the standardised criteria outlined below. Although the criteria are useful in defining neonates who may benefit from ECMO they are by no means a substitute for careful clinical assessment. It is essential that neonates referred for ECMO are considered on a individual basis. 


\subsection{SELECTION CRITERIA FOR NEONATAL ECMO}

\section{The Pre-ECMO Evaluation}

\section{i. Reversible Pathology}

ECMO itself will not heal but is an intensive care support, which allows time for lung recovery and avoidance of continuing barotrauma. ECMO will not benefit patients who have irreversible lung disease or a lethal cardiac condition. Similarly, the presence of a major congenital malformation, major chromosomal anomaly or untreatable non-pulmonary condition incompatible with a reasonable quality of life precludes ECMO treatment.

\section{ii. Neurological Status}

Infants who have suffered a severe hypoxic injury and who have associated severe hypoxic-ischaemic encephalopathy or established brain damage are excluded from receiving ECMO. The clinical evaluation prior to ECMO must include cranial ultrasound scan which although a poor method at assessing term babies is currently the best possible method for assessing babies in extremis. The cranial ultrasound scan looks specifically for lesions involving the brain parenchyma, which may be associated with poor neurological outcome. Additionally a cranial ultrasound is required to detect haemorrhage in the lateral ventricles extending beyond the subependymal or choroidal regions (greater than grade 1). Such a significant 
intraventricular bleed may extend massively after even limited heparinisation and is a contraindication to ECMO.

The presence of a grade 1 haemorrhage is a relative contraindication to ECMO and requires assessment of each individual case.

\section{iii. Gestational Age less than 34 weeks}

The requirement for systemic heparinisation during ECMO means that inevitably patients are at risk of intracranial haemorrhage. This risk is considered unacceptably high in premature infants. ${ }^{65}$ Hence gestational age $<34$ weeks is a contraindication for ECMO.

\section{iv. Birthweight greater than $2 \mathrm{Kg}$}

The restriction of birthweight is mostly related to the technical issue of the size of the available ECMO cannulae. Smaller cannulae are now being manufactured which are thinner walled with improved blood flow characteristics.

\section{v. Less than 10-14 days of mechanical ventilation}

Infants ventilated for more than 10 days with high pressure and high fractional inspired oxygen are at risk of developing bronchopulmonary dysplasia. This will eventually reach a level that delays lung recovery beyond the realistic time limits of ECMO perfusion (although these are not well defined). In clinical terms this means 
that neonates ventilated at high pressures for more than 10-14 days are excluded on the expectation of irreversible pulmonary disease.

\section{vi. Coagulopathy or uncontrolled bleeding}

Coagulopathy or bleeding is a relative contraindication to ECMO. The approach depends on the severity of the problem.

Pulmonary haemorrhage can often be controlled quite easily on ECMO. However a patient with severe diffuse intravascular coagulation (DIC) may not be suitable for ECMO due to the risk of haemorrhage with heparinisation. Each case should be considered on an individual basis. In general a cautious approach is advised in patients with a persistent elevation of the INR $>2.5$, which is not correctable by the administration of vitamin $\mathrm{K}$, or the infusion of fresh frozen plasma and cryoprecipitate.

\section{vii. The severity of illness}

Clearly, ECMO is a highly invasive specialised therapy which is not without potentially serious complications. Hence ECMO is reserved for the most severely ill patients for whom conventional treatment has failed.

In an attempt to quantify the severity of illness in neonatal intensive care a number of scoring systems have been devised. The oxygenation index (OI index) is a marker of substantial risk of death or disability. The OI index is calculated by the following

formula: $\quad$ Oxygenation Index $=\underline{\mathrm{MAP} \times \mathrm{FiO}_{2}} \underline{\underline{(\%)}}$

$$
\text { Post -Ductal } \mathrm{PaO}_{2}(\mathrm{mmHg})
$$


MAP $=$ Mean airway pressure in $\mathrm{cmH}_{2} \mathrm{O}$

$\mathrm{FiO}_{2}=$ Fractional inspired oxygen

$\mathrm{PaO}_{2}=$ Arterial oxygen concentration in $\mathrm{mmHg}$

Control studies have shown that an OI index of greater than 40 predicts a mortality of $80 \%$ for infants treated with conventional ventilation. The UK ECMO trial ${ }^{1}$ utilised an $\mathrm{OI}$ index of greater than 40 for at least 3 hours to qualify infants for randomisation. The mortality in the control infants was $61 \%$ compared to $29 \%$ for the infants who received ECMO. Thus the OI appears to be a valid marker of risk of death in such infants, although it did not predict a mortality of $80 \%$ as originally suggested. ${ }^{66}$ A more physiological measure of effective ventilation is the alveolar arterial oxygen difference. This is obtained by subtracting the arterial $\mathrm{PO}_{2}$ from the so-called "ideal" alveolar $\mathrm{PO}_{2}$. The latter is the $\mathrm{PO}_{2}$ which the lung would have if there were no ventilation-perfusion inequality and it was exchanging gas at the same respiratory exchange ratio as the real lung. It is derived from the alveolar gas equation ${ }^{67}$

$$
\begin{aligned}
& \text { Alveolar-arterial oxygen difference }=\mathrm{PaO}_{2}-\mathrm{FiO}_{2} \times(\mathrm{Patm}-\mathrm{Pwv})-\left(\mathrm{PaCO}_{2} / \mathrm{R}\right) \\
& \text { Patm }=\text { Barometric pressure }(760 \mathrm{mmHg}) \\
& \mathrm{Pwv}=\text { Pressure of water vapour }(47 \mathrm{mmHg}) \\
& \mathrm{PaO}_{2}=\text { Partial pressure of arterial oxygen } \\
& \mathrm{PaCO}_{2}=\text { Partial pressure of arterial carbon dioxide } \\
& \mathrm{R}=\text { Respiratory quotient }
\end{aligned}
$$

Values of $610 \mathrm{mmHg}$ or more, for more than 8 hours, have defined a $79 \%$ mortality in a retrospective study. ${ }^{68}$ 
The problem of carbon dioxide retention may be an indication for extracorporeal support. Attempts have been made to standardise such problems using numerical formulae. Bohn et $\mathrm{al}^{69}$ used a formula to define a population of patients with pulmonary hypoplasia who would prove irretrievable. The ventilatory index (VI) is similar to the oxygenation index but is calculated by multiplying the mean airway pressure by the respiratory rate and correlating the results with the partial pressure of arterial carbon dioxide $\left(\mathrm{PaCO}_{2}\right)$.

Congenital diaphragmatic hernia $(\mathrm{CDH})$ is the condition which remains difficult in terms of decision making for neonatal ECMO. These patients are notoriously labile and the value of predictive indices can vary greatly with time. The difficulty in managing $\mathrm{CDH}$ is due to interaction of three components in the aetiology which are the source of patient instability.

1. Pulmonary hypertension and persistent foetal circulation

2. Pulmonary hypoplasia secondary to lung compression in utero

3. The compression of existing lung tissue by hernial contents.

There are no predictors of outcome which reliably distinguish patients with $\mathrm{CDH}$ who have an inevitably fatal degree of pulmonary hypoplasia. The difficulty in managing these patients is reflected in the higher morbidity when compared with other neonatal ECMO patients.

The selection criteria for ECMO described above, define a group of mature infants who are a relatively small sub-population of neonatal intensive care patients. The most common causes of severe cardiopulmonary failure in this group are meconium aspiration syndrome, persistent pulmonary hypertension of the newborn (PPHN), 
congenital diaphragmatic hernia $(\mathrm{CDH})$, septicaemia in particular Group B streptococcus and respiratory distress syndrome. In general, they all have underlying pulmonary hypertension. The pulmonary artery pressure is increased sufficiently to cause right to left shunting across the foramen ovale and hypoxia. Oxygen is a prominent pulmonary vasodilator. Therefore, by using ECMO to oxygenate the patient this cycle can be broken and the pulmonary artery pressure reduced. 
Table 1 Contraindications to Neonatal ECMO

Absolute
- Gestational age $<34$ weeks
- Greater than Grade 1 intracranial
haemorrhage
- Severe irreversible brain injury
- Lethal malformations or lethal congenital
- Comomalies
- Significant non-treatable congenital cardiac
disease
Relative
- $10-14$ days of mechanical ventilation
- Uncontrolled bleeding or coagulopathy
- Parade 1 intracranial haemorrage




\subsection{THE OUTCOME OF NEONATAL ECMO}

The survival figures for neonatal ECMO are shown in tables 1.1 and 1.2

Table 1.1 Overall Outcome for Neonatal ECMO

\begin{tabular}{|c|c|c|c|c|}
\hline \multirow[t]{2}{*}{ Group } & \multicolumn{2}{|c|}{ Glenfield $1989-2001$} & \multicolumn{2}{|c|}{ ELSO registry July 2001} \\
\hline & Number & Survival (\%) & Number & Survival (\%) \\
\hline $\begin{array}{l}\text { Neonatal } \\
\text { Respiratory }\end{array}$ & 248 & 81 & 16488 & 78 \\
\hline $\begin{array}{l}\text { Neonatal } \\
\text { Cardiac }\end{array}$ & 14 & 57 & 1505 & 39 \\
\hline $\begin{array}{l}\text { Neonatal } \\
\text { ECPR }\end{array}$ & 1 & 100 & 62 & 40 \\
\hline $\begin{array}{l}\text { Total } \\
\text { (ECPR) Ext }\end{array}$ & $\begin{array}{l}263 \\
\text { oreal card }\end{array}$ & $\begin{array}{l}80 \\
\text { ary resuscitati }\end{array}$ & 18055 & 75 \\
\hline Survival $=s$ & to dische & nsfer & & \\
\hline
\end{tabular}

Table 1.2 Neonatal Respiratory ECMO Outcome by Diagnosis

\begin{tabular}{|c|c|c|c|c|}
\hline \multirow{2}{*}{$\begin{array}{l}\text { Neonatal Cases } \\
\text { Diagnosis } \\
\end{array}$} & \multicolumn{2}{|c|}{ Glenfield 1989 - 2001} & \multicolumn{2}{|c|}{ ELSO registry July 2001} \\
\hline & Number & Survived (\%) & Number & Survived (\%) \\
\hline Congenital diaphragmatic hernia & 45 & 60 & 3699 & 54 \\
\hline Meconium aspiration syndrome & 101 & 95 & 5831 & 94 \\
\hline Persistent pulmonary hypertension & 41 & 76 & 2382 & 79 \\
\hline Respiratory distress syndrome & 22 & 91 & 1316 & 84 \\
\hline Sepsis & 25 & 68 & 2223 & 75 \\
\hline Pneumonia & 2 & 100 & 215 & 59 \\
\hline Other & 20 & 60 & 930 & 66 \\
\hline Totals & 256 & 80 & 16596 & 78 \\
\hline Survived = Survived to discharge & & & & \\
\hline
\end{tabular}




\section{Mortality}

The UK ECMO trial $^{1}$ showed improved survival for neonatal ECMO compared to conventional treatment. The most recent data from the ELSO registry reflect a more modest survival rate than previously reported. ${ }^{70}$ This is likely to be due to the introduction of newer treatment modalities such as nitric oxide which have reduced the need for ECMO except in the more severe cases. In addition, there has been an increase in the proportion of infants in the Registry with congenital diaphragmatic hernia, who have the worst outcome.

Predictors of mortality for ECMO includes low birth weight, primary diagnosis, need for cardiopulmonary resuscitation (CPR) prior to ECMO, and severe bleeding complications on ECMO. A mortality of greater than $50 \%$ is reported for neonates with congenital diaphragmatic hernia or total anomalous pulmonary venous drainage. ${ }^{71}$ Cardiopulmonary resuscitation $(\mathrm{CPR})$ prior to ECMO has a reported increased mortality rate of $(40 \%)$ compared to infants who did not require CPR $(20 \%) .{ }^{72}$ Severe bleeding is a complication in about half the reported ECMO deaths but whether bleeding is the direct cause of death is unclear. Certainly, severe bleeding may require decannulation from ECMO at a stage earlier than the patient can survive on conventional treatment. In some instances, intractable bleeding may be an important factor for withdrawal of intensive care support.

\section{The Neurological outcome of ECMO}

Infants who require ECMO are a group of infants at high risk of brain injury. 
Neurocognitive impairment is the most prevalent handicapping condition reported for neonatal ECMO survivors. In a prospective cohort study of 103 five year old children treated with ECMO as neonates, Glass et al, ${ }^{73}$ reported the mean full scale IQs of ECMO treated children were within the normal range but as a group were significantly lower compared to normal controls. The control group consisted of 37 healthy children recruited from a local paediatric practice. A "healthy" child was defined as one who did not require admission to a special care baby unit during the neonatal period. Children treated with ECMO had an increased risk relative to the control children for academic difficulties at school age $(49 \%$ vs $22 \% \mathrm{p}<0.01)$ and a higher rate of behavioural problems reported by parents $(42 \%$ vs $16 \% \mathrm{p}=0.01)$. In the four year follow up data of infants in the UK ECMO trial, Bennett at $\mathrm{al}^{2}$ found that in assessing cognitive ability, both ECMO and conventionally treated survivors had similar scores. However, the scores for verbal, reasoning, and spatial abilities all fell below the population norms. Children were noted to have particular difficulties undertaking spatial tasks such as pattern construction and copying. Neuromotor impairment is well documented in ECMO survivors but most affected children have mild cerebral palsy with reasonable function. ${ }^{73-75}$ From estimates across a number of studies, the occurrence of severe cerebral palsy in ECMO treated neonates is less than $5 \% .{ }^{73-75}$ In the UK ECMO trial only one patient had severe disability at age one year. ${ }^{45}$ By age 4 years, the follow up study done by Bennett et $\mathrm{al}^{2}$ classified three children (all ECMO treated) as severely disabled (3\%). One severely disabled child had spastic quadriplegia and was fully dependent. A second child had hypertonia, ataxia and severe motor delay. In addition, the child suffered pituitary dysfunction and feeding was supplemented by gastrostomy. The third child was hypotonic with generalised developmental delay and solely gastrostomy fed. Two 
other children in the ECMO group required anticonvulsants and had more than six seizures in six months.

Sensorineural hearing loss has been documented in both ECMO and non - ECMO infants with similar underlying conditions (persistent pulmonary hypertension and chronic lung disease). Combining data for 313 children reported from five ECMO centres, the overall rate of auditory deficit (unilateral and bilateral) was approximately $10 \%$, with a range of $4-21 \% .^{74-78}$ The rate of bilateral hearing loss requiring amplification was $5 \%$ in the cohort study reported by Glass et al. ${ }^{73}$ In the study reported by Bennett at al, ${ }^{2} 11(12 \%)$ children had significant sensorineural hearing loss with similar proportions in both ECMO and conventionally treated survivors. Three of the children were aided and had a hearing threshold above $60 \mathrm{~dB}$. There was a tendency for thresholds to be worse toward the higher sound frequencies. Two of the three children had normal hearing during the first year of life but substantial hearing loss became apparent by 30 and 36 months respectively. Cheung et al $^{79}$ also reported progressive hearing loss in neonatal ECMO survivors. Factors which have been associated with sensorineural hearing loss in non-ECMO patients include: asphyxia, hyperventilation, hypocapnia and prolonged diuretic usage. There is no evidence to date that the ECMO procedure increases the risk for hearing impairment. This leaves us to assume those pre-ECMO factors such as hypoxia, hyperventilation and hypotension may have some contribution to later sensorineural hearing loss. The overall findings from a number of follow up studies from ECMO survivors confirm that only a minority suffers severe disability. ${ }^{73-75}$ At the present time, ECMO offers the best chance of survival for such critically ill neonates and the benefit of ECMO outweighs the risk for the majority of eligible infants. ${ }^{1-2}$ 
This said, there are a significant number of ECMO graduates who suffer mild to moderate disability. The future goal for those involved in the care of neonatal ECMO patients is to improve the neurological outcome for at least some of these survivors. Promising neural rescue therapies such as mild hypothermia may be one strategy in gaining improved neurological outcome.

\subsection{WHY THE NEED FOR NEUROPROTECTION?}

From the ECMO survivors it is not clear whether the neurological morbidity is due to ECMO itself or the severity of illness. A higher mortality has been reported in patients with congenital diaphragmatic hernia ${ }^{80}$ and bronchopulmonary dysphasia. ${ }^{81-82}$ In addition, pre-ECMO factors such as cardiopulmonary resuscitation, ${ }^{72}$ prolonged hypotension and hyperventilation are thought to contribute to the neurological problems.

Possible mechanisms of brain injury associated with the ECMO procedure include: (1) cannulation of the right common carotid artery, right internal jugular, or both resulting in changes in the cerebral blood flow; (2) the need for systemic heparinisation, which increases the risk of brain haemorrhage; (3) air or thrombic emboli, which may occur during bypass; (4) reperfusion injury to neuronal structures at the onset of bypass and following cerebral ischaemia.

It is plausible that protecting the brain in the immediate hours following cannulation for ECMO may improve the neurological morbidity in at least some of the ECMO survivors. 


\subsection{BACKGROUND FOR NEUROPROTECTION}

\section{The Concept of Neuroprotection}

\section{i. Magnetic Resonance Spectroscopy}

Magnetic resonance spectroscopy (MRS), using ${ }^{31} \mathrm{P}$ MRS can be used for the detection and quantification of intracellular $\mathrm{pH}(\mathrm{pHi})$ and the cerebral concentration of adenosine trinucleotide phosphate (ATP), phosphocreatine ( $\mathrm{PCr}$ ) and inorganic phosphate $(\mathrm{Pi})$. When ATP generation is impaired, energy flux is maintained by the breakdown of PCr whilst Pi concentration is increased. Decrease in the $\mathrm{PCr} / \mathrm{Pi}$ ratio is a valuable indicator of impaired cerebral metabolism even in the presence of normal or near normal concentration of ATP. ${ }^{31} \mathrm{P}$ MRS gives a non-invasive measurement of cerebral oxidative metabolism. In addition, proton magnetic resonance ( ${ }^{1} \mathrm{H}$ MRS) allows measurement of the lactate concentration in the brain tissue and n-acetyl aspartate which is confined to neurons and therefore may give a measurement of neuronal loss. The practical issues of MRS mean that most of the data available has been obtained from term rather than preterm infants.

\section{ii. Delayed Secondary Energy Failure}

Interest in neuroprotection was increased by the finding that although some cells die immediately following hypoxic-ischaemic injury, many more cells die hours or days later. ${ }^{83}$ In 1984, using ${ }^{31} \mathrm{P}$ MRS, Hope et $\mathrm{al}^{84}$ showed delayed cell death in birth asphyxiated neonates. Compared to normal controls, in the asphyxiated neonates the 
$\mathrm{PCr} / \mathrm{Pi}$ concentration was normal on day one of life. Thereafter, the $\mathrm{PCr} / \mathrm{Pi}$ ratio decreased signficantly in the asphyxiated infants by the second to the ninth day. In addition, the greater the decline in the $\mathrm{PCr} / \mathrm{Pi}$ ratio, the worse the neurodevelopment outcome. Increased cerebral lactate was demonstrated by Hanrahan et $\mathrm{al}^{85}$ using proton magnetic resonance spectroscopy $\left({ }^{1} \mathrm{H}\right.$ MRS $)$ to measure the lactate/creatine ratio. Delayed disruptions of cerebral energy metabolism are associated with a normal or increase in $\mathrm{pHi}$ in contrast to acute hypoxic-ischaemia when the $\mathrm{pHi}$ characteristically falls.

These findings gave rise to the concept of "secondary energy failure". Following a hypoxic ischaemic insult the cerebral cells display biphasic cellular damage. The $\mathrm{PCr} / \mathrm{Pi}$ ratio falls immediately after the insult and then returns to normal. The delayed decline in $\mathrm{PCr} / \mathrm{Pi}$ ratio then begins about 8-12 hours after birth asphyxia and continues for hours or days later. It is not contingent on continued hypoxia or intracellular acidosis. These findings have been confirmed and further quantified by other workers. $^{86-87}$

The clinical signficance of delayed energy failure is that the greater the decline in the $\mathrm{PCr} / \mathrm{Pi}$ ratio, the worse the developmental outcome at one and four years. Infants with secondary energy failure show reduced head growth velocity, implying abnormal brain development. ${ }^{88-89}$

\section{iii. Hypoxic-ischaemic Brain Injury in Immature Animals}

Studies in animal models have found similar biphasic changes in cerebral energy metabolism following global ischaemia in piglets and focal stroke in rat pups. Lorek et $\mathrm{al}^{90}$ used phosphorus magnetic resonance during their investigation of hypoxic 
ischaemia in newborn piglets. During hypoxic-ischaemia there was a decrease in the phosphocreatine concentration [PCr]/ inorganic orthophosphate concentration $[\mathrm{Pi}]$ and nucleotide triphosphate concentration $[\mathrm{NTP}] /$ exchangeable phosphate pool concentration $[\mathrm{EPP}]$ ratio. On reperfusion and reoxygenation both ratios returned to the baseline. Over the next 48 hours continuous monitoring showed that the $\mathrm{PCr} / \mathrm{Pi}$ ratio again decreased in spite of normal arterial $\mathrm{PO}_{2}$ and mean arterial blood pressure. $\mathrm{NTP} / \mathrm{EPP}$ ratio also decreased but to a lesser extent. Intracellular $\mathrm{pH}$ remained unchanged. In rat pups, Palmer et $\mathrm{al}^{91}$ demonstrated the phosphocreatine $(\mathrm{PCr})$ and adenosinetriphosphate (ATP) levels decreased to 14 and $26 \%$ of the controls at the end of hypoxia-ischaemia. During the first hour of recovery, the mean PCr was replenished to within $90 \%$ of baseline whereas the mean ATP was incompletely restored to $68-81 \%$ of the control $(\mathrm{p}<0.05)$. Yager et $\mathrm{al}^{92}$ also showed decrease and failure of recovery of phosphocreatine (PCr) and adenosine triphosphate (ATP) following focal stroke in rat pups. In both models the more severe the hypoxicischaemia the greater the magnitude of the secondary energy failure and the worse the histological outcome.

Studies of foetal sheep have provided further evidence of the biphasic changes in cerebral metabolism following hypoxic-ischaemia. Williams et $\mathrm{al}^{93}$ used electroencephalography (EEG) quantified with real-time spectral analysis to demonstrate changes during and after hypoxic-ischaemia. Similar to the experiments in piglets and rat pups, there was a dose response relationship between the length of time of ischaemia and the period of EEG depression. In sheep subjected to 10 or 20 minutes ischaemia, EEG activity was depressed and then progressively recovered and mild selective neuronal loss was seen on histology. After 30 or 40 minutes of ischaemia, there was a sequence of prolonged EEG depression followed by 
epileptiform activity, and then loss of EEG intensity. This sequence was associated with laminar necrosis of the cortex on histological assessment and an increase in the concentrations of excitatory amino acids and nitric oxide metabolites in the CFS. ${ }^{94}$

\section{iv. Issues Regarding the Concept of Delayed Secondary Energy Failure}

The concept of delayed secondary energy failure has provided a vehicle to highlight the progressive nature of cerebral injury caused by hypoxic-ischaemia. However, more recent experiments would suggest that post-hypoxic changes in the brain are more complex. ${ }^{95}$

First, experiments by Thornton et $\mathrm{al}^{96}$ using diffusion-weighted magnetic resonance (DWMR) have shown that the changes in brain tissue following hypoxic-ischaemia are not uniform. Magnetic resonance measurement of the apparent diffusion coefficient (ADC) of brain tissue water gives an index of the restriction of water and thus of membrane integrity. The changes in ADC following ischaemia closely paralleled the changes in $\mathrm{PCr} / \mathrm{Pi}$ ratio during delayed secondary energy failure. However the changes in ADC are not uniform throughout the brain. The abnormal diffusion begins laterally and then progresses to involve the more central regions. This implies that a global change in $\mathrm{PCr} / \mathrm{Pi}$ ratio does not reflect the complexity of regional differences in tissue injury.

Second, magnetic resonance imaging of infants who develop secondary energy failure after hypoxic-ischaemia have shown heterogeneous damage throughout the brain. Damage to the basal ganglia and deep brain structures seem to predict a worse neurological outcome. ${ }^{97}$ Also, magnetic resonance imaging has also shown regional differences in cerebral metabolite concentrations; for example lactate is reported to be 
higher in the thalamus than the occipital region. ${ }^{98}$ Again, these regional changes must be considered with the concept of delayed energy failure.

Third, it is now clear that following hypoxic-ischaemia the cerebral metabolic changes can be detected for much longer periods than was previously known. Using magnetic resonance, Hanrahan et $\mathrm{al}^{86}$ demonstrated a persistent increase in cerebral lactate for many months in infants who developed neurodevelopment impairment. The mechanism for this extreme prolongation of secondary energy failure is unknown but again highlights the complexity of the metabolic changes following hypoxicischaemia.

Despite these complexities, delayed cerebral energy failure is a valuable concept. This value is reflected in experiments using neural rescue therapies. Treatments, which reduce the delayed secondary energy failure and cell death, show improvement in histological outcome. However, treatments, which do not reduce cell death also have no effect on the delayed secondary energy failure. (SEE LATER SECTION)

\subsection{MECHANISM OF DELAYED CELL DEATH}

\section{Apoptosis in Hypoxic-ischaemic Cerebral Injury}

The mechanism of delayed cell death following hypoxic-ischaemic cerebral injury is unclear. However, in the brains of infants dying after birth asphyxia cells can be detected with features of apoptosis. ${ }^{99}$ This has led to the hypothesis that inappropriate activation of the apoptotic programme accounts for at least part of the delayed cell death seen after transient hypoxia-ischaemia. 
Further evidence has been reported to support this hypothesis. Features of apoptosis have been observed following hypoxic-ischaemic injury in piglets, ${ }^{100}$ and both adult $^{101}$ and immature rats. ${ }^{102-103}$

\section{Apoptosis and Necrosis}

Necrosis is in general, a form of cell death that rapidly occurs due to an overwhelming insult and is typically associated with changes in the calcium and sodium ion homeostasis. ${ }^{104}$ In contrast, apoptosis is a delayed form of cell death that results from a less severe insult and is associated with activation of a "genetic programme". The early morphological changes of necrosis result from disruption of the plasma membrane ionic pump and include cellular and organelle swelling, especially the mitochondria. ${ }^{105}$ Organelles become disrupted and finally there is cell lysis, with spillage of the cytoplasmic contents into the extracellular space. The cell debris is removed by phagocytosis which results in an inflammatory response. ${ }^{104}$ Apoptotic cells undergo a very different process, which commences with shrinkage of the cytoplasm and condensation of the nuclear material into "clumps". 106-107 The nuclear membrane then folds around the fragments of DNA and the plasma membrane invaginates resulting in cytoplasmic vacuolation. In the final stages, the cell separates into multiple discrete apoptotic bodies. These bodies are phagocytosed, mainly by neighbouring healthy cells with little or no inflammatory response. Apoptosis is an active cellular process that requires specific biochemical pathways, energy consumption and sometimes gene transcription.

Apoptosis is distinguished from necrosis by electron microscopy. However, electron

microscopy has a limited application in quantification studies of apoptosis. ${ }^{108}$ DNA 
fragmentation during cell death is another means of distinguishing necrosis from apoptosis. In necrosis, DNA undergoes random fragmentation following histone proteolysis whereas during apoptosis, the histones remain intact hence the DNA is cleaved into multimers of $180-240$ base pairs. ${ }^{109}$

This difference in DNA fragmentation has been used in an attempt to distinguish necrotic from apoptotic cells. Using gel electrophoresis, genomic DNA from necrotic cells exhibits a single vertical band on electrophoresis ("smear pattern"). In contrast, in apoptosis, genomic DNA exhibits a "ladder pattern" on electrophoresis. However, due to potential technical difficulties, especially with DNA resolution it is generally difficult to differentiate these two patterns.

In situ DNA fragmentation can be detected by labelling of the terminal deoxynucleotidyl transferase-mediated niick end labelling (TUNEL). This involves binding of dUTP tagged with a chromagen or fluorophore to the 3 ' $-\mathrm{OH}$ ends of single or double-stranded DNA. ${ }^{110}$ Although this method is quite sensitive for detection of in situ DNA fragmentation, it cannot differentiate necrotic and apoptotic cells.

During the early stages of apoptosis, phosphatidyl-serine is translocated from the inner surface to the outer surface of the plasma membrane. ${ }^{111}$ The calcium-dependent phospholipid binding protein, annexin $\mathrm{V}$, can bind to phosphatidylserine after its translocation to the external surface of the cell. ${ }^{111}$ The use of fluorophore-tagged annexin and a membrane excluded dye, such as propidium iodide provides a method of detecting apoptotic cells. Given the limitations of the methods available, quantitative apoptotic studies are best performed using electron microscopy in conjunction with methods such as TUNEL and Annexin V to quantify apoptotic cells. $^{108}$ 


\section{Apoptosis in the Multicellular Organism}

In normal circumstances, cells will undergo programmed cell death (apoptosis) unless they receive "survival signals" from neighbouring cells. ${ }^{112}$ Experiments reported by Raff showed that cells in culture would universally undergo apoptosis unless either they were maintained at high density or the culture medium was artificially supplemented with appropriate trophic factors. ${ }^{112}$ This suggested that proteins, normally growth factors such as insulin -like growth factor -1 (IGF-1) or members of the neurotrophin family are secreted in an autocrine or paracrine manner, and are essential to prevent execution of the apoptotic death programme.

The death of cells deprived of signals from their neighbours provides a mechanism for both the control of normal morphogenesis and the prevention of cancers: a cell population, which grows beyond its "correct" size or location, will run out of trophic factors and die. An example is the formation of digits from the primitive limb buds. Brain cells which fail to make proper synaptic connections die by apoptosis and as many as $50 \%$ of cells in some parts the brain die in this way during normal cerebral development. $^{113}$

\section{Apoptosis in Disease}

In addition to being a primary mechanism for regulating normal development, apoptosis is involved in the pathogenesis of many diseases. A number of disorders have been identified that involve excess apoptotic cell death. These include AIDS, where lymphocyte death is triggered by HIV; neurodegenerative disorders, such as 
spinal muscular atrophy and Parkinson's disease; and hypoxic-ischaemic cerebral damage. $^{114}$

\section{Triggers for Apoptosis}

Apoptosis is triggered by a number of physiological and pathological stimuli: trophic factors such as IGF-1bind to tyrosine kinase receptors and loss of these factors probably cause apoptosis by removing phosphorylation signals. ${ }^{112}$ Apoptotic death is often preceded by the induction and activation of a variety of genes including immediate-early genes (IEG's), Bcl-2 (and related proteins) and the p53 gene.

Immediate early genes (IEGs), c-fos, fos B, c-jun, jun-D, krox-20, krox-24, NGFI-B Nurr 77/TIS1, and NGFI-C are oncogenes and related genes that produce nuclear proteins functioning as transcriptional activators of other genes. The transcriptional activity of IEGs is regulated by phosphorylation by a number of protein kinases. Following hypoxic-ischaemic brain injury, rapid induction and transcriptional activation of IEGs occur in response to increased intracellular calcium, excitatory amino-acid exposure, electrical depolarisation and oxygen free radical exposure. ${ }^{115}$ 117

The induced IEGs may activate a number of genes encoding structural proteins, enzymes, ion channels or neurotransmitters resulting in long-lasting changes in neuronal structure and function. ${ }^{118}$ It is unclear whether these changes in IEG expression promote or inhibit apoptosis in neurons. It is most likely that IEGs both inhibit and promote apoptosis depending on their interaction with other proteins. 
Under basal conditions, the p53 gene exists in low concentrations within cells and has a half-life of $\sim 5-20$ minutes. ${ }^{119-120}$ In response to DNA damage, cellular levels of p53 increase (mainly by translation) and the gene is translocated to the nucleus where it transcriptionally activates a number of target genes. ${ }^{121}$ The target genes include, bax, IGF-BP-3, p2 ${ }^{\text {WAF1/Cip1 }}$, GADD45, cyclin G and mdm2. These activated proteins serve a number of functions including the induction of apoptosis, cell cycle regulation, DNA repair and autoregulation of $\mathrm{p} 53$.

The bcl-2 family is a group of proteins that regulate cell death. Bcl-2 and bcl-xl are complex proteins localised to the membranes of the endoplasmic reticulum, nuclear envelope and the outer mitochondrial membrane. ${ }^{122-124}$ The bcl-2 channel is selective for potassium while bcl-xl channel conducts sodium. ${ }^{125-126}$ Both bcl-2 and bcl-xl regulate the mitochondrial permeability transition pore (PT) and the release of proteins from the mitochondrion eg cytochrome $c$, which are critical to the activation of cellular proteases. ${ }^{127}$

The mitochondrial PT pore is a large $(\sim 2.9 \mathrm{~nm})$ cyclosporin - sensitive channel present in the inner mitochondrial membrane and opens during the process of apoptotic cell death. A high concentration of calcium ions and reactive oxidant species (ROS) in the mitochondria triggers opening of the pore and allows oxygen free radicals to enter the cytoplasm. While transient opening of the PT pore may be involved in calcium homeostasis or free radical release, sustained opening of the PT pore causes uncoupling of respiratory enzymes, failure of ATP generation and the release of specific apoptosis-initiating proteins such as cytochrome-c into the cytoplasm. 
$\mathrm{Bcl}-2$ and bcl-xl regulate the opening of the PT pore, preventing the release of free radicals and inhibiting apoptosis. The exact mechanism of how bcl-2 acts on the PT pore is unclear.

Bax is a member of the bcl-2 family which promotes apoptosis. The Bax channel is mainly chloride selective and lies within the mitochondrial membrane. Bax binds with other members of the bcl-2 family leading to loss of mitochondrial membrane potential, cytochrome-c release and caspase activation.

Cells in the immune system undergo apoptosis when activated by a cell surface receptor, Fas/Apo-1 ${ }^{127}$, also, tumour necrosis factor- alpha (TNF- $\alpha$ ) activates sphingomyelinase, leading to an increase in cytosolic concentration of ceramide, a potent inducer of apoptosis. ${ }^{128}$

\section{Mechanism of Apoptosis}

The intracellular mechanism of apoptosis has not been fully established. Since death is the default pathway of all cells, the apoptotic cascade pathway is permanently ready to act and in some cases can proceed in the absence of de novo messenger RNA or protein synthesis. The release of apoptogenic proteins such as cytochrome $\mathrm{c}$ into the cytoplasm, triggers a cascade leading to apoptotic execution. Cytochrome $\mathrm{c}$ forms a complex with the protein Apaf-1 which in turn forms a complex with caspase 9 . The formation of an Apaf-1/caspase -9 complex activates caspase-3, which then triggers apoptotic execution, by activating downstream caspases and endonucleases. The process is dependent on cytochrome $\mathrm{c}$ and ATP, although there is some evidence that apoptosis can proceed in the absence of cytosolic translocation of cytochrome C. ${ }^{129-130}$ 


\section{Mechanism of Apoptosis Following Hypoxic-ischaemic Injury}

The mechanism by which transient hypoxic-ischaemia induces apoptosis is unknown. There are possibly several overlapping pathways by which hypoxic-ischaemia can induce apoptosis including, DNA damage, increased intracellular calcium concentration or other forms of intracellular disorganisation. Agents of hypoxicischaemic damage such as glutamate can induce apoptosis. Glutamate is an excitatory amino acid released in high quantities at the nerve endings during hypoxia. It is now known that the mitochondrion is central to glutamate-induced cell death. ${ }^{131}$ In the early stages of glutamate-induced apoptosis, calcineurin, a calcium-calmodulinregulated phosphatase is activated within the cytoplasm by glutamate. This allows calcineurin to dephosphorylate the protein bad which is translocated to the mitochondrial membrane to dimerise with either bcl-xl or bcl-2, a process that initiates apoptosis. ${ }^{132}$ It is this dimerization that induces the release of cytochrome c from the mitochondria and, along with Apaf-1 leads to caspase activation. ${ }^{133}$ Alternatively, glutamate can activate bax in a p53-mediated process. The translocation of bax to the mitochondrial membrane and its destabilising effect are important factors that influence mitochondrial function during apoptosis. Once bax translocates to the mitochondrial membrane, it crosslinks as a homodimer leading to loss of mitochondrial membrane potential, cytochrome $\mathrm{c}$ release and caspase activation. 


\subsection{THE CONCEPT OF THE “THERAPEUTIC WINDOW” FOR NEUROPROTECTION}

The finding of delayed cell death following hypoxic -ischaemia has given rise to the concept of a "therapeutic window" of opportunity for neuroprotection. The proposed hypothesis being that applying a neuroprotection strategy in the latent period following the hypoxic-ischaemic insult may prevent delayed cell death and reduce the cerebral damage.

This has led to a huge body of research investigating both the mechanisms of delayed cell death and potential neural rescue therapies. From the results of animal studies, mild hypothermia appears to be a promising means of neuroprotection. ${ }^{3-4}$ However, before discussing the evidence for mild hypothermia as a neuroprotective strategy, I will first discuss some of the other experimental neural rescue therapies.

\subsection{NEURAL RESCUE THERAPIES}

\section{Excitatory Amino Acid Antagonists}

Normal brain function requires a balance between excitatory and inhibitory neurotransmitter activity. The amino acid glutamate is one of the major excitatory neurotransmitters ${ }^{134}$ and there is evidence that glutamate plays an important role in the mechanism of hypoxic-ischaemic injury. Firstly, glutamate is directly toxic to mature

neurones in culture. ${ }^{135}$ Secondly, neurons in culture within hippocampal slices die on exposure to anoxia (nitrogen), but their death can be prevented or attenuated by the presence of magnesium, which blocks glutamate receptors, or by specific glutamate 
receptor antagonists. ${ }^{136}$ Thirdly, direct injection of glutamate or glutamate agonists into specific regions of the brain produces neuronal injury identical to that seen after hypoxic-ischaemic injury. ${ }^{137-138}$

In a study reported by Szatkowski et al ${ }^{139}$ two distinct phases of glutamate release were noted to produce neuronal death. During hypoxic -ischaemia, glutamate is released by reversed operation of glutamate uptake carriers, activating the N-methylD-aspartate (NMDA) receptors, displacing magnesium ions that block the ion channel, and allowing calcium influx, thus increasing the intracellular concentration of calcium. Glutamate which is released following hypoxic-ischaemia, activates excessive influx of calcium through potentiated NMDA-receptor channels. This leads to a cascade of damaging biochemical events. Activation of the amino-3-hydroxy-5methyl-4-isoxazole-propionic acid (AMPA) receptors contributes to the intracellular accumulation of calcium via sodium influx and membrane depolarisation. In terms of pharmacological agents which may offer neuroprotection, there are two potential approaches to inhibit the neurotoxic effect of glutamate. One approach would be to prevent the release of glutamate from the axon terminal into the postsynaptic cleft which occurs during hypoxic-ischaemia. Agents such as baclofen which prevent the release of glutamate from the neuronal ending, have not been investigated as potential neuroprotective agents. The second approach would prevent the excessive stimulation of the cell receptors on the surface of the neurone by glutamate, which promotes neuronal death. Agents which antagonise the NMDA receptors, AMPA receptors or the calcium ion channels involved in their functioning have received most attention. The compounds studied include Dizocilpine (MK-801), phencycline, dextromorphan amongst others. ${ }^{140}$ 
NMDA and AMPA receptor antagonists have been shown to reduce neuronal death both in adult and immature animal models even when applied after the insult. McDonald et al $^{141}$ showed that systemic administration of MK -801 prevented tissue necrosis produced by the direct injection of NMDA into the striatum of 7-day old rat pups. Dizocilpine (MK-801) has been shown to protect against hypoxic-ischaemic cerebral damage in immature rats, even when administered up to one hour following the hypoxic-ischaemic insult. ${ }^{142-144}$ In foetal sheep, Dizocilpine (MK-801) given 6 hours after severe hypoxic-ischaemia was seen to abolish seizure activity and provided a moderate degree of neuroprotection, particularly in the hippocampus and lateral cortex. ${ }^{144}$

The therapeutic benefit of some drugs has not been universal, depending in part on the type of model under investigation. ${ }^{140}$ In addition, most of the studies have focused on areas of brain rich in glutamate receptors e.g. hippocampus. It remains unclear whether NMDA antagonists will offer neuroprotective benefit in the areas of the brain with few or no glutamate receptors. An example of this differential neuroprotective effect was shown in a study of focal ischaemia in rodents reported by McAuley. ${ }^{145}$ In this model of focal ischaemia, NMDA antagonists typically halved the area of damage, but the protection was maximal in the cortex and minimal in the striatum. This difference may be due to differing numbers of glutamate receptors or perhaps differing degrees of hypoxic insult.

Phase III clinical trials of NMDA receptor antagonists as a treatment for stroke in adults were commenced in the 1990 's, ${ }^{146}$ but few have shown a clear neuroprotective benefit. Therefore enthusiasm for NMDA receptor antagonists as a neural rescue therapy has to a certain extent diminished. However, investigation into the expressive 
profile of the postischaemic NMDA receptor subtypes may give insight into new strategies.

\section{Magnesium}

Magnesium is known to have a role in the gating of $N$-methyl-D-aspartate (NMDA) receptors and this has led to interest in its potential as a neural rescue therapy following hypoxic-ischaemia. However, studies in newborn animals have given conflicting results with regard to the neuroprotective effect of magnesium sulphate. In a study reported by Nakajima et $\mathrm{al}^{147}$ magnesium sulphate was found to inhibit the dopamine surge during anoxia in rat pups which was thought to be at least partially neuroprotective. In a study of 7 day -old rat pups, magnesium sulphate given 15 minutes after intracerebral injection of NMDA resulted in reduced cerebral injury. ${ }^{148}$ Similarly, in 5 -day old mice, reduced cerebral damage was reported when magnesium sulphate was given before or 10 minutes after an injection of ibotenate, a glutamate antagonist, into the brain. ${ }^{149}$ However, magnesium sulphate given after transient hypoxia-ischaemia failed to prevent delayed cerebral energy failure in newborn piglets. ${ }^{150}$ In a further study in newborn piglets, Greenwood et al ${ }^{151}$ reported that following hypoxic -ischaemia there was no difference in the severity of cerebral damage between piglets treated with magnesium sulphate and the placebo group. Similarly, magnesium sulphate was not found to be neuroprotective in foetal lambs following hypoxia and/or ischaemia. ${ }^{152}$ In clinical practice, a retrospective epidemiological study by Nelson et al ${ }^{153}$ suggested that antenatal exposure to magnesium reduced the incidence of cerebral palsy at age three years. Randomised controlled double-blind trials were established to investigate 
this hypothesis but one has been discontinued after interim analysis showed that magnesium sulphate given to mothers in preterm labour before 34 weeks was associated with increase in foetal deaths. ${ }^{154}$ However, interim results from other trials have not shown any difference in the mortality rates between the placebo and treatment groups. ${ }^{155}$ An international network, headed by Levene et al ${ }^{156}$ was involved in a randomised clinical trial to investigate whether magnesium sulphate is neuroprotective after birth asphyxia. However, due to a problem with the dosage of magnesium sulphate the trial has been discontinued. At the present time, there is insufficient data to recommend magnesium sulphate as a neural rescue therapy.

\section{Nitric Oxide Synthase Inhibitors}

As discussed in section 1.7, the mechanisms underlying delayed cerebral energy failure are unclear, but some evidence suggests that excessive production of nitric oxide may be involved. ${ }^{157-159}$ Nitric oxide production increases after hypoxicischaemia, ${ }^{94,160}$ studies have shown that nitric oxide can act as a potent inhibitor of mitochondrial function ${ }^{161-163}$ and can trigger both necrotic and apoptotic cell death by different mechanisms. ${ }^{164}$ In addition, excessive nitric oxide produced following hypoxic -ischemia can rapidly react with superoxide radicals to form peroxynitrite. Peroxynitrite and other related reactive nitrogen species (RNS) produce nitration of lipids, DNA and proteins, thereby irreversibly modifying brain cell constituents. ${ }^{165}$ Nitric oxide synthase (NOS) enzymes involved in nitric oxide production exist in three different isoforms: the constitutive endothelial NOS (eNOS), neuronal NOS (nNOS) and the inducible NOS (iNOS). All NOS isoforms are upregulated after hypoxia-ischemia but in different time profiles. ${ }^{166}$ 
A number of authors have considered the potential neuroprotective effect of reducing nitric oxide production using nitric oxide synthase inhibitors but with varying results. In a study of fetal sheep subjected to transient ischaemia and then treated with $N^{\mathrm{G}}$ nitro-L-arginine (L-NNA) (a nitric oxide synthase inhibitor), Marks et al reported worsening cerebral injury. This may have been as a consequence of disruption of cerebral haemodynamics. ${ }^{167}$ However, studies of knock out mice, genetically lacking neuronal nitric oxide synthases, showed these mice to be less vulnerable to hypoxiaischaemic injury. ${ }^{168}$ More recently, Peeters-Scholte et al ${ }^{169}$ reported neuroprotection by selective nitric oxide synthase inhibition 24 hours after perinatal hypoxicischaemia in newborn piglets. In this study, simultaneous and selective inhibition of neuronal and inducible nitric oxide synthase by 2 - iminobiotin resulted in $90 \%$ reduction in vasogenic oedema, a $90 \%$ improvement in the cerebral energy state and a $60-80 \%$ reduction in apoptosis-related neuronal cell death.

The exact mechanism by which inhibition of nitric oxide synthase causes neuroprotection is not certain. A study of 14 day old rat pups reported by Blumberg et $\mathrm{al}^{170}$ found that nitric oxide synthesis was not involved in delayed cerebral energy metabolism. Therefore, the neuroprotective effects of selective NOS inhibitors is likely to be mediated by other mechanisms such as the reduction of reactive nitrogen species (RNS). ${ }^{165,169}$

\section{Oxygen Free Radical Inhibitors and Scavengers}

Although not fully understood, it appears that most of the tissue injury caused by hypoxic -ischaemic cerebral injury occurs following completion of the insult during 
the reperfusion period. A damaging cascade of reactions is then set in motion, including the release of excitatory amino acids, and the generation of free-radicals. Important tissue free radicals include superoxide anion $\left(\mathrm{O}^{2-}\right)$, hydroxyl radical $(\mathrm{OH})$, nitric oxide and other reactive oxygen species, including hydrogen peroxide $\left(\mathrm{H}_{2} \mathrm{O}_{2}\right)$ and peroxynitrite. Under normal conditions, these toxic species are produced by cellular metabolism and neutralised primarily by the combined action of three enzymes: superoxide dismutase (SOD), glutathione peroxidase (GPx), and catalase (Cat). During the reperfusion period, an overproduction of reactive oxygen species overwhelms the cellular anti-oxidant capacity. These free radicals may then attack cellular components, causing peroxidation of membrane lipids and damage to DNA and protein, resulting in neuronal injury and cell death. In addition, activated microglia and macrophages, appearing in the brain 24 hours after the initial injury, generate free radicals and contribute to delayed neuronal death. Furthermore, during cerebral hypoxia-ischaemia, mitochondrial oxidative phosphorylation is impaired, causing adenosine troposphere (ATP) degeneration and accumulation of hypoxanthine. ${ }^{171}$ Hypoxanthine is metabolised by the enzyme xanthine oxidase to xanthine and uric acid in reactions which produce super oxide and hydrogen peroxide. Allopurinol, an inhibitor of the enzyme xanthine oxidase, blocks the synthesis of xanthine from hypoxanthine and, therefore prevents the formation of free radical superoxide. ${ }^{172-173}$ It is not known whether allopurinol inhibits circulating xanthine oxidase or endogenous xanthine oxidase in the brain, or both. ${ }^{173}$

In experimental animal models, administration of allopurinol to rat pups thirty minutes before inducing focal hypoxic-ischaemia reduced the severity of the extent of the neuropathological lesion and lessened cerebral oedema in the treated group compared to the control group. ${ }^{174}$ Similarly, pre-treatment with allopurinol preserved 
cerebral energy metabolism of the 7-day postnatal rat during hypoxia-ischaemia. ${ }^{175}$ Oxypurinol, the active metabolite of allopurinol, also administered pre - insult to immature rats has also been shown to reduce brain injury in the immature rat. ${ }^{176}$ Clinically, a 7-day course of enteral allopurinol $(20 \mathrm{mg} / \mathrm{kg})$ given after birth to 400 infants between 24 and 32 weeks' gestation, did not change the incidence of periventricular leukomalacia. ${ }^{177}$ In a study of 22 asphyxiated newborns, intravenous allopurinol in a dose of $40 \mathrm{mg} / \mathrm{Kg}$ given 4 hours after birth resulted in a decrease mortality (2/11 versus $6 / 11$ in the control group), and showed a beneficial effect on free radical formation, cerebral blood flow, and electrical brain activity, without toxic side effects. ${ }^{178}$ To date there have been no published results of a multicentre trial investigating the value of allopurinol in reducing postasphyxial brain injury.

\section{Free Radical Elimination}

Superoxide dismutase (SOD) and catalase are intracellular enzymes that contribute to the cell's ability to detoxify super oxide and hydrogen peroxide, respectively. Superoxide dismutase and catalase are large molecules that do not easily penetrate cell membranes or the blood-brain barrier. They cannot be pharmacologically effective without gaining access to the intracellular sites of free radical generation but endothelial cell and blood-brain barrier penetration of SOD can be achieved by encapsulation with phospholipid liposomes.

Both animal experimental models and clinical investigation of the neuroprotective potential of exogenously administered antioxidant enzymes have been reported. Imaizumi et al ${ }^{179}$ used liposome-entrapped SOD to reduce infarct volume following middle cerebral artery occlusion in rats. 
Conjugation of SOD to polyethylene glycol (PEG-SOD) blocks renal clearance, increases its circulating half-life, endothelial cell penetration, and oxidant resistance. PEG-SOD and PEG catalase pre-treatment has been shown to reduce infarct volume following middle cerebral artery occlusion in adult rats. ${ }^{180}$ Superoxide and hydrogen peroxide are considered weakly reactive oxygen species and are not considered capable of causing significant tissue injury alone. Rather, they react with transition metals such as iron to form more reactive species like the hydroxyl radical. The newborn infant may be particularly susceptible to free radical injury because of a relative deficiency in brain superoxide dismutase ${ }^{181}$ and the relative inability to sequester iron. ${ }^{182}$ At the present time there are no clinical trials of SOD in asphyxiated neonates.

\section{Antioxidants: Free Radical Scavengers}

\section{Vitamin E}

Vitamin E or alpha - tocopherol is a membrane bound, chain breaking antioxidant. Once lipid peroxidation is initiated, peroxyl radicals react with vitamin E instead of an adjacent fatty acid, thus terminating the process. ${ }^{183}$

Vitamin $\mathrm{E}$ is the principal antioxidant in the lipid enviromnent. In adult animals deprived of vitamin $\mathrm{E}$ in their diet, Van der Worp et al ${ }^{184}$ reported a worse neurological outcome following cerebral ischaemia. In a rat model of focal ischaemia, the administration of vitamin $\mathrm{E}$ reduced the infarct volume by $81 \% .{ }^{185} \mathrm{In}$ vitro studies have shown that vitamin $\mathrm{E}$ is necessary for the survival of cultured 
neurones from fetal brain. ${ }^{186}$ Also, vitamin E prevents apoptosis in cortical neurones during hypoxia and reperfusion. ${ }^{187}$

In the clinical setting, vitamin $\mathrm{E}$ has been used in an attempt to prevent intraventricular haemorrhage in preterm infants. One of the problems of using vitamin $\mathrm{E}$ as a neural rescue therapy is that it is lipid soluble and for an effective dose to reach the brain or circulation, vitamin E needs to be given for some days before the ischaemic insult. $^{173}$

\section{Lazeroids}

The lazeroids are nonglucocorticoid, 21 -aminosteroids with potential neuroprotective properties. Tirilazad mesylate is a member of this class of compounds which prevents iron-dependent lipid peroxidation by scavenging peroxyl radicals. In focal models of hypoxic - ischeamia, tirilazad mesylate (U-74006F) has been shown to prevent lipid peroxidation and significantly improve the recovery of calcium homeostasis, ${ }^{188}$ prevent postischemic cerebral oedema ${ }^{189}$ and reduce neuropathological injury in 7 day old rat pups. ${ }^{190}$

Lazeroids have shown promise particularly in the treatment of acute spinal cord injury. ${ }^{191}$ However, there have as yet been no randomised controlled trial of the use of lazeroids for either acute spinal injury or neonatal hypoxic-ischaemic cerebral injury.

\section{Antiapoptotic Agents}

As discussed in section 1.7, apoptosis is a highly regulated mechanism of cell death with a characteristic morphology. Apoptosis appears to account for at least part of the 
mechanism of delayed cell death following hypoxic-ischaemic cerebral injury. After initiation of the apoptotic program, the time after which a cell becomes irreversibly committed to cell death can vary from a few minutes to several weeks. ${ }^{192}$ The decision to commit is affected by a delicate balance between pro- and anti apoptotic forces within the cell.

The proto-oncogene bcl-2, has been shown to prevent cell death triggered by free radicals, glucose deprivation, excess glutamate, and growth factor deprivation. The prevention of neuronal death induced by such diverse insults suggests that bcl-2 intervenes at a physiological step common to both apoptotic and necrotic neuronal death pathways, perhaps by the neutralisation of reactive oxygen species. ${ }^{193-194} \mathrm{Bcl}-2$ has demonstrated to be neuroprotective following hypoxia-ischaemia. In transgenic mice that over express bcl-2, the size of the infarct caused by ischaemia is significantly smaller. ${ }^{195}$ The use of viral vectors such as herpes simplex virus (HSV) allows the over expression of exogenous genes in post mitotic cells such as neurons. ${ }^{196}$ The over expression of bcl-2 using herpes simplex viral vectors has shown to limit neuronal death when administered prior to or following focal cerebral ischaemia. $^{193,196}$

Antiapoptotic agents are still in the experimental stages of investigation and are not yet clinically proven. Much of the current work is focusing the importance of apoptosis and necrosis in perinatal brain injury and determining to what degree apoptosis is a final common pathway for neural cell death. 


\section{Vitamin C}

Vitamin $\mathrm{C}$ or ascorbic acid is believed to act as a neuromodulator that facilitates the release of neurotransmitters and inhibits neurotransmitter binding to NMDA. ${ }^{173}$ Vitamin $\mathrm{C}$ is one of the principal antioxidant systems in the fetus, and enzymatic antioxidant systems do not develop until late in the third trimester. ${ }^{197}$ Plasma vitamin $\mathrm{C}$ is maintained at high level in fetal blood and declines rapidly after birth, perhaps as a result of increased oxidant production, increasing the risk of oxidant injury. Vitamin $\mathrm{C}$ levels in the brain are approximately 10 - fold those in the plasma; they are well maintained during global ischaemia and there is a transient decrease during ischemia-reperfusion. ${ }^{173}$ In animal models, vitamin $\mathrm{C}$ has been shown to have a neuroprotective effect in the adult rat hippocampus following kainate induced neurotoxicity. ${ }^{198}$ Also, in a neonatal rabbit model, administration of vitamin $\mathrm{C}$ to the dam resulted in an increase in cellular viability in the cortex and hippocampus. ${ }^{199}$

The optimal dose of vitamin $\mathrm{C}$ for neuroprotection is not yet established. In in vitro studies high dose vitamin $\mathrm{C}$ has been shown to cause death in cortical neuronal cells. ${ }^{200}$ Vitamin $\mathrm{C}$ administered to high - risk premature infants has been shown to be safe. ${ }^{201}$ However, there is no further clinical data at present to confirm vitamin $\mathrm{C}$ as more than a potential neural rescue therapy.

\section{Poly(ADP-ribose) Polymerase}

Poly(ADP-ribose) polymerase (PARP) plays a physiological role in the repair of strand breaks in DNA. ${ }^{202}$ It requires nicotinamide adenine dinucleotide $\left(\mathrm{NAD}^{+}\right)$as a 
cofactor, and excessive PARP activity, can lead to cell death through depletion of energy stores. Administration of a PARP inhibitor led to a significant reduction in infarct volume following focal ischaemia in rats $^{203}$ and a genetic deletion of PARP in mice attenuated tissue damage following a similar injury. ${ }^{204}$

\section{Growth Factors}

Many factors are important for normal growth and maturation of the brain. A number of growth factors have been shown to be induced following brain injury, including transforming growth factor $-\beta_{1}$, basic fibroblast growth factor, nerve growth factor (NGF) and insulin-like growth factor (IGF-1). Many of these growth factors have neuroprotective activity and may prove to be useful as a neural rescue therapies. For example, in a neonatal rat model of hypoxia-ischaemia, intracerebroventricular administration of nerve growth factor (NGF) decreased significantly the amount of damage in both the striatum and cortex ipsilateral to the carotid ligation (10\% volume loss versus $35 \%$ in the control group). ${ }^{205}$ Further studies have shown that NGF markedly protects against hypoxic-ischaemic cerebral injury at post-natal day $7,90 \%$ when given prior to the insult and 50\% when given after the insult. In contrast, NGF has a less neuroprotective effect when administered on post-natal day 21 , suggesting that NGF may have a different effect on the immature compared with the adult brain. $^{206-207}$

Insulin-like growth factor (IGF-1) has also been shown to have a neuroprotective effect. In rats and fetal sheep, central administration of IGF-1 after transient hypoxicischaemia reduced both neuronal loss and the incidence of cortical infarction in a dose-dependent manner. The neuroprotective effects of IGF -1 have been shown to be 
particularly strong in the striatum. ${ }^{207-209}$ The neuroprotective effects of IGF-1 seems to be selectively related to cholinergic and certain populations of GABAergic neurons. ${ }^{210}$

\section{Calcium Channel Blockers}

Experimental evidence supports a major role for increased intracellular calcium levels in the induction of neuronal damage during cerebral ischaemia. ${ }^{172-173,197,211}$ Increased cytosolic calcium activates lipases, proteases and endonucleases, which attack the structural component of the cell; the activation of phospholipases produce a breakdown in the phospholipid components of the plasma and subcellular membranes. Calcium channel blockers inhibit the intracellular entry of calcium during ischaemia and may augment cerebral blood flow during ischaemia and during the reperfusion period, therefore maintaining neuronal viability. ${ }^{172-173,183,212-213}$

\section{Calcium Channel Blocker - Flunarizine}

In an animal model of focal ischaemia, flunarizine has been shown to be neuroprotective in 7 and 21-day-old rats if given prior to but not after the hypoxicischaemic insult. ${ }^{214-215}$ In adult gerbils, subjected to global ischaemia, flunarizine at $0.5 \mathrm{mg} / \mathrm{Kg}$ decreased neuronal loss and improved morbidity, but flunarizine at 1.0 $\mathrm{mg} / \mathrm{Kg}$ did not. ${ }^{216}$ Clinically, flunarizine has been investigated as a neuroprotective agent in adult stroke. In a large multicentre trial, flunarazine was given within 6 or 24 hours after acute hypoxic-ischemia in the distribution of the middle cerebral artery. The results of the study showed no improvement in neurological or functional outcome in the patients treated with flunarazine versus the control group. ${ }^{217}$ 


\section{Calcium Channel Blocker - Nimodipine}

In adult rats subjected to partial reversible focal neocortical ischaemia, nimodipine given by intra - arterial injection before and during the insult has been shown to decrease the infarct volume. ${ }^{218}$ However, nimodipine at a dose of $10 \mathrm{mg} / \mathrm{Kg}$ did not protect against neuronal loss in adult gerbils subjected to transient bilateral carotid artery occlusion. ${ }^{219}$ In newborn piglets with mild hypoxic - ischaemia, nimodipine caused a reduction of regional cerebral blood flow, blood pressure and cardiac output. $^{220}$ Thus it remains to be clarified as to whether nomodipine is efficacious in preventing or reducing the extent of perinatal hypoxic-ischaemic damage in experimental animals.

Clinical trials of nimodipine in adult stroke patients showed decreased mortality and improved neurological prognosis, including improvement of memory scores. ${ }^{221-222}$

\section{Calcium Channel Blocker - Nicardipin}

Levene et $\mathrm{al}^{223}$ reported four term infants with severe asphyxia. The medication caused significant hypotension; all four patients died.

\section{Iron Chelators}

Hydrogen peroxide $\left(\mathrm{H}_{2} \mathrm{O}_{2}\right)$ is an oxygen free radical produced by tissue metabolism and as a by-product of superoxide anion metabolism. During hypoxic-ischaemia, particularly in the immature brain, $\mathrm{H}_{2} \mathrm{O}_{2}$ may cause damage either directly, or perhaps more importantly, through its conversion in the presence of ferrous ions to the highly 
reactive hydroxyl $(\mathrm{OH})$, the Fenton reaction. Production of hydroxyl $(\mathrm{OH})$ ion via the Fenton reaction may then initiate a chain of further damaging reactions to which the immature brain is particularly susceptible, such as lipid peroxidation, and protein and DNA oxidation.

Iron chelators such as deferoxamine have been shown in some systems to inhibit lipid peroxidation and hydroxyl radical production via the Fenton reaction and to reduce hypoxic-ischaemic and reperfusion associated brain injury. In a study using a neonatal mouse model of hypoxic-ischaemia, Sarco et al reported a significant decrease in cerebral injury in mice treated with deferoxamine ten minutes after the hypoxicischaemic insult. $^{224}$ In a separate study reported by Palmer et al, deferoxamine given post-treatment reduced ischaemic brain injury in neonatal rats. ${ }^{225}$

At the present time only experimental data exists for iron chelators as a neuroprotective strategy following hypoxic-ischaemic cerebral injury. It has not yet been proven as a clinically effective agent.

\section{Monosialogangliosides}

Monosialogangliosides $\left(\mathrm{GM}_{1}\right)$ are found in high concentrations in the brain and are important constituents of cellular membranes. ${ }^{173.226}$ In studies of fetal sheep, treated with $\mathrm{GM}_{1}$ pre- and post hypoxic-ischaemic brain damage, reduction in neuronal

injury was seen in the cerebral cortex, hippocampus and striatum. ${ }^{227-228}$ A randomised controlled trial of post insult $\mathrm{GM}_{1}$ in adult stroke patients showed improved neurological outcome after 4 months with no apparent adverse drug effects. $^{229}$ 
The exact neuroprotective mechanism of $\mathrm{GM}_{1}$ following cerebral hypoxic-ischaemic injury is unknown. It is possible that $\mathrm{GM}_{1}$ is incorporated into cellular membranes results in a stabilisation of membrane integrity and function. ${ }^{226}$ In addition, $\mathrm{GM}_{1}$ ganglioside inhibits ischaemic release of amino acid neurotransmitters from rat cortex. ${ }^{230}$ Clinical trials of monosialogangliosides following neonatal hypoxicischaemic cerebral injury are still awaited.

\subsection{MILD HYPOTHERMIA AS A NEUROPROTECTIVE STRATEGY}

A number of studies in immature animals have shown that a reduction of the body temperature by $3^{0} \mathrm{C}$ or $4^{0} \mathrm{C}$ after hypoxic-ischaemic cerebral injury has a number of neuroprotective effects including:

1. preservation of cerebral energy metabolism

2. reduction in cytotoxic oedema

3. improved histological and behavioural outcome.

In 1995, Thoresen et $\mathrm{al}^{3}$ reported amelioration of delayed cerebral energy failure in newborn piglets when mild hypothermia was applied following severe transient hypoxia-ischaemia. In this study, six piglets underwent temporary occlusion of the common carotid arteries and hypoxia. Resuscitation was started when cerebral phopshocreatine $[\mathrm{PCR}]] /[$ inorganic phosphate $[\mathrm{Pi}]$ ratio as determined by phosphorous magnetic resonance spectroscopy had fallen to almost zero and the nucleotide troposphere [NTP]/exchangeable phosphate pool [EEP] had fallen below about $30 \%$ of baseline. The piglets were immediately whole body cooled to $35^{\circ} \mathrm{C}$ for 12 hours and then rewarmed to normothermia $\left(38.5^{\circ} \mathrm{C}\right)$. Spectroscopy results over the next 64 hours were compared with previously established data from 12 piglets 
similarly subjected to transient cerebral hypoxia-ischemia, but maintained normothermic, and six sham operated controls. The mean severity of the primary insult (judged by the time integral of depletion of $([\mathrm{NTP}] /[\mathrm{EPP}])$ was similar in the hypothermic and normothermic groups. In the normothermic group, $[\mathrm{PCR}] /[\mathrm{Pi}]$ and $[\mathrm{NTP}] /[\mathrm{EPP}]$ recovered from the acute insult and then fell again. In the hypothermic group, a large reduction in delayed cerebral energy was demonstrated and no fall in either $[\mathrm{PCR}] /[\mathrm{Pi}]$ or $[\mathrm{NTP}] /[\mathrm{EPP}]$ occurred up to 64 hours after the hypoxicischaemic insult.

In immature rats a number of studies have shown benefit of cooling following an hypoxic-ischaemic insult. In a study of 21 day old rat pups, Sirimanne et $\mathrm{al}^{5}$ showed improved histological outcome in rats maintained at $22^{\circ} \mathrm{C}$ for 72 hours compared with rats held at an environmental temperature of $34^{\circ} \mathrm{C}$. In an experiment using 7 day old rat pups reported by Thorensen et $\mathrm{al}^{231}$, hypoxic -ischaemia was induced by ligation of the left carotid artery followed by 120 minutes of normothermic exposure to $8 \%$ oxygen. The rat pups were then randomised either to three hours of hypothermia $\left(32.5^{0} \mathrm{C}\right)$ or normothermia. The animals treated with hypothermia had significantly less brain damage than normothermic animals. Interestingly, in a similar study in seven day old rat pups, Yager et $\mathrm{al}^{232}$ showed a neuroprotective effect if cooling was applied during but not immediately after hypoxic-ischaemia. One explanation for the difference in findings may be that all the animals in the Yager study had a rectal probe insitu and were restrained. This may have caused increased stress and modulated the neuroprotective effect.

In terms of behavioural outcome, in a study of seven-day old rat pups, Bona at $\mathrm{al}^{233}$ reported four tests of sensorimotor function which would allow the long term evaluation of neurological function following hypoxic-ischaemic injury. The 
neurological assessment done five - six weeks after the insult showed significant neurological impairment in the hypoxic - ischaemic animals compared with controls. Colbourne et $\mathrm{al}^{6}$ described a neuroprotective effect in gerbils cooled following a five minute occlusion of the cerebral artery. Following ischaemia, the gerbils were randomised to three treatment groups. One hour post-ischaemia, group 1 were cooled to $32^{\circ} \mathrm{C}$ for 24 hours, in group 2 cooling to $32^{\circ} \mathrm{C}$ was initiated 4 hours post-ischaemia and continued for 24 hours. A final group was cooled to $34^{\circ} \mathrm{C}$ one hour postischaemia and continued for 24 hours. A control group was maintained at $37^{\circ} \mathrm{C}$ following ischaemia.

Six months following the experiments, the surviving gerbils were assessed both using learned behaviour testing and the brains examined histologically. Compared to the control groups, gerbils cooled to $32^{\circ} \mathrm{C}$ one hour post ischaemia had a significant reduction in loss of CA1 neurons and a less impaired learning in an open field and T-

maze. Animals treated with mild hypothermia $\left(34^{0} \mathrm{C}\right)$ showed neuroprotective benefit but the effect was less than in the moderate hypothermia group.

\section{How does Hypothermia Work?}

The exact mechanism by which hypothermia induces neuroprotection is unclear. However, there are several potential mechanisms which may contribute to some degree to the neuroprotective effect of cooling. Hypothermia applied during hypoxic-ischaemia is thought to be protective by preventing the decline in high energy phosphates which seem to initiate both apoptotic and necrotic cell death. ${ }^{234-235}$ 
The mechanism by which cooling after hypoxic-ischaemic injury prevents cell death is less clear. Hypothermia prevents the delayed decline in phosphocreatine and adenosine triphosphate, as well as the simultaneous increase in cerebral lactate concentration, seen 8-12 hours after hypoxic-ischaemia in newborn piglets. However, it is not clear whether the preservation of cellular energy metabolism following hypoxic-ischaemic injury is the main mechanism by which hypothermia operates or whether it represents an indicator of cellular protection mediated by other pathways. $^{236}$

An additional effect of hypothermia is a reduction in extracellular glutamate. Hypoxic-ischaemic injury results in high concentrations of glutamate in the synaptic cleft. Glutamate is neurocytotoxic and induces excitotoxic neuronal death. In both newborn piglets ${ }^{161}$ and adult rats ${ }^{237}$ hypothermia reduces the delayed increase in extracellular glutamate seen after hypoxic-ischaemia. Also, mild hypothermia has been shown in vitro to decrease the impairment in glutamate reuptake which is an important factor in cellular injury. ${ }^{238}$ Hypothermia also reduces the nitric oxide production which itself is neurocytotoxic and contributes to neuronal death. ${ }^{161.239}$ Hypothermia may also increase catecholamine secretions ${ }^{240}$ and since stress has shown an improved neuropathological outcome in immature rats sympathetic stimulation may be another factor in neuroprotective effect of hypothermia. ${ }^{241}$ There is a suggestion from the results of experiments on newborn piglets that hypothermia may specifically inhibit apoptosis. ${ }^{242}$ However, in cell culture studies mild hypothermia delays rather than prevents apoptosis. ${ }^{243}$ Edwards et al $^{236}$ hypothesised that hypothermia delays commitment to apoptosis for long enough to enable endogenous protective mechanisms, including the production of growth factors, to be induced. ${ }^{244-245}$ 
Studies of adult animals have suggested other mechanisms through which hypothermia may act including: suppression of free radical action; prevention of ischaemia induced protein kinase $\mathrm{C}$ inhibition; or activation of transcription factors. ${ }^{246}$ It is not clear whether these mechanisms are important in the immature brain. It seems likely that hypothermia has a neuroprotective effect via several mechanism of damage, which are activated by hypoxia-ischaemia.

\subsection{THE CHALLENGES OF APPLYING MILD HYPOTHERMIA IN A}

\section{CLINICAL SETTING}

Translating these promising experimental findings to successful application in human subjects poses a number of challenges including:

i.The timing of hypothermia following hypoxic-ischaemic injury?

ii..The optimal length of cooling to achieve neuroprotection?

iii.The optimal cooling temperature to achieve neuroprotection?

iv. Whole body cooling versus local head cooling

v.The potential adverse effects of cooling

\section{i. Timing of Applying Mild Hypothermia Following Hypoxic-Ischaemic Cerebral Injury}

In a clinical setting the practicalities of firstly identifying neonates who have suffered significant hypoxic-ischaemic cerebral injury will inevitably mean a delay of perhaps several hours before applying mild hypothermia. This has prompted suggestions that 
the delay in applying a neural rescue therapy may result in the loss of any potential neuroprotective effect.

However, there is some evidence that the pathological postasphyxial process may not be confined to the period immediately after injury. In proton magnetic resonance $\left(\mathrm{H}^{1} \mathrm{MRS}\right)$ studies reported by Hanrahan et al, ${ }^{86}$ eighteen neonates with birth asphyxia were studied. Ten neonates with mild asphyxia had normal development at one year and no signal of cerebral lactate was detected. Eight neonates suffering birth asphyxia had abnormal development at one year and cerebral lactate was detected in 7 of the 8 infants greater than one month after birth.

In a study in 14 day old rat pups, Taylor et $\mathrm{al}^{247}$ showed improved neurological outcome in rat pups cooled between 6-12 hours after an hypoxic-ischaemic insult. In this experiment using an established neonatal model of focal hypoxic-ischaemia, rat pups were either kept normothermic or cooled to $30^{\circ} \mathrm{C}$ for 6 hours commencing at either $0,2,4$, or 6 hours after the end of hypoxia. Twenty four hours after hypoxicischaemia, cerebral energy metabolism was measured by phosphorous magnetic resonance spectroscopy, and at 5 days cerebral infarction size was measured. Shamoperated animals were used as controls. In normothermic animals the ratio of phosphocreatine/inorganic phosphate $(\mathrm{PCr} / \mathrm{Pi})$ had fallen markedly 24 hours following hypoxic-ischaemia. In contrast, animals cooled between 6 and 12 hours displayed high $\mathrm{PCr} / \mathrm{Pi}$ ratios similar to those in control animals. Similarly, after 5 days, infarct area was significantly reduced only in animals cooled between 6 and 12 hours after injury. These finding suggest that cellular events occurring 6 to 12 hours after hypoxic-ischaemia are susceptible to modulation by hypothermia. This has implications for the practical application of hypothermia as a neural rescue therapy in 
birth asphyxia and suggests that there is adequate time for diagnosis before treatment is started, without loosing the neuroprotective effect of the therapy.

\section{ii. The Optimal Length of the Cooling Period}

The results of animal experiments clearly show a neuroprotective effect of cooling, however systematic data on the optimal duration of cooling is incomplete. Protection has been seen in studies of immature animals with cooling periods varying between 3 and 72 hours ${ }^{4,231}$ A longer cooling period was shown to be beneficial in 14 day old rat pups cooled for up to 6 hours following hypoxia-ischaemia. ${ }^{247}$

Although the possibility of adverse effects of cooling are greater with prolonged cooling, intuitively most researchers feel that a longer cooling time must be more beneficial in terms of optimal neuroprotective effect. Also the evidence of

postasphyxial changes evident months after the hypoxic-ischaemic insult ${ }^{86}$ lends more support to the theory that cooling times of perhaps longer than 72 hours may be beneficial.

Further experimental work is needed in this particular area of research before a definitive answer is known.

\section{iii. Whole Body Systemic Cooling Versus Selective Head Cooling}

It is not clear from the available experimental evidence whether systemic whole body cooling or selective local head cooling is better.

Proponents of selective head cooling argue that using this technique, neuroprotection can be achieved but with fewer systemic adverse effects. 
The counterclaim of advocates of whole body cooling is that by this method, the deep brain structures, in particular the basal ganglia may be more adequately cooled. Magnetic resonance imaging (MRI) studies have shown that infants with basal ganglia injury have worse neurological impairment than those with cortical damage alone. $^{97}$

The question remains whether local head cooling alone can achieve adequate deep brain cooling without at least some measure of systemic hypothermia. There is some evidence from animal experiments that head cooling alone may be successful, but in some cases temperature gradients were significant and quite extreme surface cooling was required ${ }^{4,231}$

In a study comparing brain temperature during local head cooling or whole body cooling in newborn piglets, Laptook et $\mathrm{al}^{248}$ showed that head cooling resulted in an increase in the temperature gradient across the brain with the deep brain warmer than the superficial brain. To achieve modest reductions in brain temperature $\left(\sim 2-3^{0} \mathrm{C}\right)$ of deep cortical and thalamic structures, the surface of the brain had to be cooled by almost $10^{\circ} \mathrm{C}$. Whereas body cooling to a rectal temperature of $34^{\circ} \mathrm{C}$ did not change the temperature gradient between the dura and deep brain. Given the extent of head surface hypothermia necessary to cool the deep brain structures, Laptook et $\mathrm{al}^{248}$ suggested that a mild reduction in body temperature would facilitate some cooling of the deeper brain. Consistent with these findings, is the heat sink model applied to a 3dimensional model of the infant head to examine temperature distributions in response to surface cooling to $10^{\circ} \mathrm{C} .{ }^{249}$ The model demonstrated that surface cooling of the head reduces brain temperature only when core body temperature was lowered. Interestingly, in the study reported by Laptook et $\mathrm{al}^{248}$ when hypoxia was superimposed during head cooling, there was an elevation in brain temperature at all 
sites, with the largest increases, $\sim 4^{0} \mathrm{C}$, noted for the superficial brain. In contrast, hypoxia superimposed during whole body cooling did not change the brain temperature. This finding has possible implications for applying cooling in the clinical setting since factors such as hypoxia may result in an increase in the brain temperature and may modify the effect of hypothermia as a neural rescue therapy.

In contrast, Thoresen et $\mathrm{al}^{250}$ demonstrated that selective head cooling could be achieved without significantly cooling the rest of the body. In this experiment, nine newborn piglets were anaesthetised and brain temperatures measured at the surface and in the superficial and deep brain matter. The piglets underwent a 45-minute hypoxic-ischaemic insult. To assess the impact of the hypoxic insult on the effectiveness of selective head cooling, four piglets (group A) were cooled both before and after the insult, and four (group B), only after the insult. One piglet followed the same cooling pattern as group A but the rectal temperature was allowed to fall lower before warming. Selective head cooling was achieved using a cooling cap. During selective head cooling, it was possible to achieve a lower deep brain than rectal temperature in all animals before and after hypoxia. However, it was essential to use an overhead heater in combination with head cooling, to create a substantial rectal to deep brain temperature gradient, since head cooling alone led to total body cooling.

Ultimately, the question of mode of cooling will be difficult to resolve until measurement of the deep brain temperature can be achieved in clinical practice. Noninvasive measurement of deep brain temperature can be achieved using magnetic resonance techniques ${ }^{251,252}$, but these are too cumbersome for routine, repeated clinical use. Measurements based on the zero-flux method have been attempted but 
not validated. ${ }^{253,254}$ A portable non-invasive deep brain thermometer based on microwave technology is underdevelopment but is not yet available. ${ }^{255}$ In conclusion, the possibility of higher brain temperature during hypoxia must be considered when planning trials of cooling as a neuroprotective strategy.

\section{Recent Clinical Trials of Mild Hypothermia}

There are to date two published trials of mild hypothermia following neonatal hypoxic-ischaemic encephalopathy.

In the CoolCap Study, (Gluckman et $\mathrm{al}^{256}$ ) neonates with hypoxic-ischaemic encephalopathy were randomised to either conventional care or mild hypothermia by local head cooling. The patients were randomised on the basis of a number of study inclusion criteria including amplitude integrated electroencephalographic (aEEG) changes. In the conventionally treated group, $73 / 110(63 \%)$ compared to $59 / 108$ $(55 \%)$ of cooled patients died or suffered severe disability by 18 months (OR 0.61 ; 95\% CI 0.34-1.09 $\mathrm{p}=0.1$ ). Head cooling was found to be beneficial in infants with moderate aEEG changes $(n=172$ OR $0.42 ; 95 \%$ CI $0.22-0.8 \mathrm{p}=0.009)$. However, hypothermia had no effect in infants with the most severe aEEG changes.

In another study by Shankeran et $\mathrm{al}^{257}$, neonates were randomised with hypoxicischaemic encephalopathy were randomised to either conventional treatment or whole body hypothermia. Death or severe disability occurred in 45 of 102 patients (44\%) in the hypothermia group and 64 of 103 patients (62\%) in the control group (risk ratio $0.72 ; 95 \% \mathrm{CI} 0.54-0.95 ; \mathrm{p}=0.01)$. Twenty four $(24 \%)$ of the infants in the hypothermia group and $38(37 \%)$ in the conventionally treated group died (risk ratio $0.68 ; 95 \% \mathrm{CI} 0.44-1.05 ; \mathrm{p}=0.08)$. The rate of cerebral palsy was 15 of $77(19 \%)$ in 
the hypothermia group versus 19 of $64(30 \%)$ patients in the control group (risk ratio $0.68 ; 95 \%$ CI $0.38-1.22 ; \mathrm{p}=0.2)$

\subsection{ADVERSE EFFECTS OF MILD HYPOTHERMIA}

Applying mild hypothermia to neonates receiving ECMO has potential adverse effects both for the patient and on the functioning of the ECMO circuit as detailed below:

\section{Potential Adverse Effects in the Patient}

\section{i. Coagulation System Effects}

Hypothermia-induced coagulation abnormalities include reversible platelet sequestration and dysfunction, enhanced fibrinolytic activity and slowing of enzymatic activity required for clotting. ${ }^{258}$

Temperature affects the prothrombin time (PT), thrombin time (TT), and partial thromboplastin time (PTT). At $34^{\circ} \mathrm{C}$, PT and PTT increase by $9 \%$ compared to $37^{0} \mathrm{C}^{13,259}$

Platelet function appears to be adversely affected by mild hypothermia. In a study of baboons, surface cooling to $32^{\circ} \mathrm{C}$ resulted in a significant increase in the bleeding time due to inhibition of thromboxane B2 production. ${ }^{14}$ Platelet dysfunction was reversed by rewarming to normal temperature $\left(37^{0} \mathrm{C}\right)$. Reversible inhibition of platelet activation by hypothermia in vivo and in vitro was also demonstrated by Michelson et al. ${ }^{260}$ In this experiment, platelet activation was studied in normal volunteers by whole blood flow cytometric analysis of modulation of platelet surface GMP-140 and the 
glycoprotein (GP) Ib-IX complex in: a) shed blood emerging from a standardised in vivo bleeding time wound; b) peripheral blood activated in vitro with either thrombin or the stable thromboxane analogue (TX) $\mathrm{A}_{2}$ analogue U46619. Platelets in the peripheral whole blood were activated at temperatures between $22^{\circ} \mathrm{C}$ and $37^{\circ} \mathrm{C}$. Platelet aggregation was studied in shed blood by flow cytometry and the peripheral blood by aggregometry. Generation of $\mathrm{TXB}_{2}$ (the stable metabolite of thromboxane) was determined by radioimmunoassay. In vitro and in vivo, hypothermia $\left(22^{\circ} \mathrm{C}-\right.$ $32^{\circ} \mathrm{C}$ ) inhibited platelet activation as shown by the inhibition of both thrombin and thromboxane (TX) $A_{2}$ analogue (U46619) induced upregulation of GMP-140, downregulation of the GPIb-IX complex, reduction in both platelet aggregate formation and thromboxane $\mathrm{B}_{2}$ generation. These inhibitory effects of hypothermia were all completely reversed by rewarming the blood to $37^{\circ} \mathrm{C}$.

In a study of hypothermic pigs $\left(30^{\circ} \mathrm{C}\right)$, Oung et $\mathrm{al}^{15}$ found that the bleeding time was nearly doubled, however, rewarming deaths were attributed to hyperaggregation of platelets and resultant massive haemorrhage. ${ }^{261}$

Mild to moderate hypothermia has been implicated in increased perioperative bleeding. In a study reported by Schmied et $\mathrm{al}^{262}$ patients were randomly assigned to normothermia or mild hypothermia during elective hip arthroplasty. A reduction of just $1.6^{\circ} \mathrm{C}$ core temperature increased blood loss by $500 \mathrm{mls}(30 \%)$ and significantly augmented allogeneic transfusion requirements. In a series of trauma patients reported by Ferrara et al ${ }^{263}$ eighty percent of nonsurviving trauma patients were hypothermic compared to $36 \%$ of survivors. Non-survivors were more severely hypothermic $\left(31.0^{\circ} \mathrm{C} \pm 1.0^{\circ} \mathrm{C}\right.$ vs. $\left.34.0^{\circ} \mathrm{C} \pm 0.5^{\circ} \mathrm{C}\right)$ and developed clinically significant bleeding despite adequate blood component replacement when compared to survivors. 
The fibrinolytic system is involved in the regulation of blood clot formation, with the conversion of plasminogen to plasmin at the core of the fibrinolytic mechanism.

Preliminary data suggests that fibrinolysis remains normal during mild hypothermia but is significantly increased during hyperthermia, suggesting that hypothermiainduced coagulopathy does not result from excessive clot lysis. The corresponding effects of thermal disturbances on plasminogen activator have yet to be determined, but thromboelastographic data suggest that hypothermia impairs clot formation rather than facilitating clot degeneration. ${ }^{264}$

\section{ii. Circulatory Effects}

The circulatory effects of hypothermia include increased peripheral vascular resistance and cardiac afterload. During cooling, there is a shift of fluid from the intravascular space causing a mild increase in blood viscosity and haematosis. ${ }^{265-266}$ Chen et $\mathrm{al}^{267}$ reported a $4-6 \%$ increase in blood viscosity for each $1^{0} \mathrm{C}$ in temperature reduction.

\section{iii. Cardiac Effects}

Hypothermia may be associated with myocardial ischaemia. In a prospective, randomised study, Frank et $\mathrm{al}^{268}$ demonstrated that high risk patients assigned to only a $1.3^{\circ} \mathrm{C}$ core temperature reduction were three times as likely to experience adverse myocardial outcomes.

The mechanism by which mild hypothermia triggers myocardial events remains unclear. Cold-induced hypertension in the elderly is associated with a three fold 
increase in plasma norepinephrine concentrations, ${ }^{269}$ which may increase cardiac irritability and lead to the development of ventricular arrhythmias.

Hypothermia also causes hypertension in elderly patients and those at high risk for cardiac complications. ${ }^{240}$ In a study reported by Green et a ${ }^{270}$ hypothermia of $34^{0} \mathrm{C}-$ $35^{\circ} \mathrm{C}$ markedly decreased porcine left ventricular contraction. Mild hypothermia has also been shown to reduce neonatal cardiac output (by $39 \%)^{271}$ and impair diastolic relaxation. Also, the efficacy of cardiovascular medications may be impaired during mild hypothermia. Riishede et $\mathrm{al}^{272}$ reported that the chronotropic effect of dobutamine on the contraction velocity of isolated perfused and spontaneously beating rabbit hearts was substantially reduced at $32^{\circ} \mathrm{C}$.

Widening of the QT interval during deep accidental or induced hypothermia is well described and may be a precursor of cardiac arrhythmias including the potentially lethal torsade de pointes. ${ }^{273-274}$

Of the few human studies that have investigated the cardiac effects of prolonged mild hypothermia none have reported cardiac arrhythmias ${ }^{275-277}$ However, mild hypothermia may cause conduction disturbances and ventricular irritability by potentiating other drugs such as bupivacaine ${ }^{7}$ or by causing hypokalaemia. ${ }^{278}$ Mild hypothermia shifts the potassium intracellularly and predisposes to hypokalaemia with related complications in postsurgical patients. ${ }^{278}$ Also, the toxicity of potassium administration appears to be potentiated by hypothermia. Sprung et $\mathrm{al}^{279}$ observed that the rat cardiac toxicity of intravenously administered potassium progressively increased with falling temperature. At $31{ }^{\circ} \mathrm{C}$, toxicity was $5 \%$ higher than at $37^{\circ} \mathrm{C}$. Aggressive potassium replacement during hypothermia can furthermore result in hyperkalaemia on rewarming. ${ }^{280}$ 


\section{iv. Immunological Effects}

A further potential adverse effect of mild hypothermia is immune suppression resulting in an increased risk of infection during cooling. There is evidence that mild hypothermia reduces T-cell mediated antibody production ${ }^{281-282}$ and impairs chemotaxis and phagocytic engulfment of bacteria by polymorphonuclear leukocytes. $^{11}$

Also, mild hypothermia has been implicated in facilitating the development of perioperative wound infections. ${ }^{283}$ In the postoperative period, mild hypothermia is thought to have a twofold effect. Firstly, hypothermia triggers thermoregulatory vasoconstriction $^{284}$ which decreases skin oxygen delivery. Second, mild hypothermia impairs various immune functions, including leucocyte moblility and phagocytosis. ${ }^{11}$ In a study of guinea pigs, Sheffield et $\mathrm{al}^{10}$ reported a decrease in the resistance to dermal infection in the animals subjected to mild hypothermia $\left(36^{\circ} \mathrm{C}\right)$ compared to the normothermic animals $\left(39^{\circ} \mathrm{C}\right)$.

\section{v. Neurological Effects}

Hypothermia is known to reduce intra-cranial pressure which is generally an advantage in head injured patients. With each degree of temperature reduction, cerebral blood flow (CBF) and cerebral metabolic rate of oxygen $\left(\mathrm{CMRO}_{2}\right)$ decrease approximately $5 \%{ }^{285}$ During deep hypothermia in monkeys, Steen et $\mathrm{al}^{286}$ reported that cerebral blood flow (CBF) distribution was inhomogeneous and failed to return to baseline on rewarming. Steen et al, ${ }^{287}$ further observed cardiovascular collapse with severe hypoxia and acidosis on rewarming similarly cooled dogs. Although these 
findings were at lower temperatures, it is possible that prolonged mild hypothermia may have at least some influence on the cerebral blood flow.

\section{vi. Metabolic Effects}

Decreasing the body temperature lowers the metabolic rate by approximately $5 \%-7 \%$ per ${ }^{0} \mathrm{C}^{265}$ Hypothermia also shifts the haemoglobin-oxygen dissociation curve to the left, thus reducing tissue oxygen availability. This may contribute to an uncompensated metabolic acidosis which is commonly observed with hypothermia. ${ }^{278}$

\section{Potential Adverse Effects on the ECMO Circuit Function}

With regard to applying prolonged mild hypothermia during ECMO the effects of cooling on the coagulation system may potentially affect the ECMO circuit function in a number of ways.

Firstly, ECMO patients are routinely heparinised with the potential for bleeding a recognised complication of ECMO. Hence, any disturbance of coagulation caused by mild hypothermia may increase the risk of bleeding.

Also, increased blood viscosity during cooling may increase the tendency to form blood clots within the circuit. Blood clots may lodge within the membrane oxygenator with the potential to cause failure of the oxygenator. In addition, blood clots could potentially enter the return cannula causing embolic phenomena in the patient.

Clearly, mild hypothermia has the potential to adversely affect a number of systems however, the neuroprotective benefit of cooling following hypoxic-ischaemic cerebral injury may outweigh the adverse effects of prolonged mild hypothermia. Neonates 
receiving ECMO represent a group at high risk of hypoxic ischaemic brain injury. Clearly this situation is not entirely analogous to perinatal asphyxia but it does seem plausible that protecting the brain immediately following cannulation for ECMO may prevent further cerebral injury. However, in view of these potential adverse effects a pilot study to assess the feasibilty of applying mild hypothermia during neonatal ECMO was necessary before considering a randomised controlled trial to assess the neuroprotective effect of cooling.

\subsection{THE ACCURACY OF LABORATORY MEASUREMENTS DURING COOLING}

One of the most difficult challenges in obtaining meaningful data during this study was the fact that routine laboratory investigations were performed at $37^{\circ} \mathrm{C}$. A particular example of this problem is the measurement of coagulation during cooling. Routine laboratory coagulation profiles were measured at $37^{\circ} \mathrm{C}$ yet during cooling the steady state temperature was below this. The concern was that any effect of cooling on the particular measurement may be masked on rewarming the blood.

In attempt to gain a more accurate picture of the effect of cooling, I used a number of laboratory investigations which were independent of temperature. These specialised laboratory tests are explained in more detail in the following chapters.

In addition, a number of other physiological parameters were measured including amplitude intergrated electroencephalography (aEEG) and the QTc interval using digital electrocardiography (ECG). These were used to gain additional information on the systemic effects of mild hypothermia. 


\section{CHAPTER 2: ETHICS}

\section{ISSUES OF PARENTAL CONSENT}

The majority of infants referred for ECMO treatment are transferred from hospitals outside the Leicester region. The retrieval team has only limited time at the referral centre since the infants are often in extremis and require urgent transfer to the ECMO centre. The parents are not permitted to accompany the infant during the transfer for reasons of safety and limited space in the ambulance or helicopter. Hence information must given within a restricted time. Once the parents arrive at the ECMO centre, more detailed discussions can then take place.

Written consent is required both for the transfer from the referral centre and ECMO treatment. The parents are therefore given a considerable amount of new information about their child's condition, the concept of ECMO and its complications. This information is given at a time when the parents are under a great deal of emotional stress. Usually the mother has just recently given birth and may be recovering from an anaesthetic. Often the retrievals take place during the night so that the parents are approached for consent when they have had little or no sleep. In spite of these difficult circumstances the majority of parents are required to make a relatively speedy decision due the critical condition of their child.

The research was approved by the Leicester Health Authority Ethical Committee. Ethical approval was given for the first stage of the study (cooling for 24 hours) on the basis that I did not need to seek written consent. The feeling was that giving the parents information about the hypothermia study at the time of the retrieval would be too burdensome. Also there was a question as to whether informed consent for clinical 
trials can be obtained under such stressful conditions. ${ }^{288-289}$ Instead, ethical approval was given for cooling to be considered as a modification of ECMO management. However I was required to give the parents an information sheet about the study and discuss any queries regarding the work.

By the time of the second stage of the study (five infants cooled for 48 hours), the climate had changed particularly regarding issues for obtaining consent. The Leicester Health Authority Ethical Committee gave approval for the second stage of the study but written consent was required prior to cooling.

In practice, my preferred approach was to endeavour to inform the parents about the research prior to initiating cooling. To achieve this aim I attended the majority of retrievals of the infants included in the study. Whilst at the referring hospital, I spent the time available talking to the parents discussing both ECMO and the hypothermia study. I gave the information sheet to the parents at this time. Although not seeking written consent I did give the parents an opportunity to decline to be included in the study. I also emphasised that they could still withdraw from the study once they had had time to read and absorb the information on the sheet. I followed up this initial interview by further explanation of the study and discussion with the parents after the infant was stabilised on ECMO.

In the few cases where I was not able to attend the referring hospital, the Consultant in charge of the retrieval spoke briefly to the parents about the research and ascertained whether they had a major objection to being included in the study. The Consultant also reiterated that I would explain the research in more detail once the parents had arrived at the Glenfield Hospital. I then attempted to speak to the parents about the study at the earliest opportunity, which in the majority of cases was on day one of the admission. 
For the patients in group 5 (48 hour cooling), I attended all the retrievals and gained written consent for the study from the parents prior to commencing cooling. I also gave the parents an information sheet about the research and updated them about the research on a regular basis during the study period.

Only one family out of 32 families approached refused consent for the study. No parents requested that their child be withdrawn from the study once it was commenced.

\subsection{INDEPENDENT DATA MONITORING COMMITTEE}

Given that we did not need written consent for the first stage of the study, an Independent Data Monitoring Committee was established as a separate safeguard. I reported to the Committee on a case by case basis. Ultimately the Committee had the authority to insist that the research be discontinued should there have been problems related to cooling at any stage. Although written consent was sought for stage two, the Data Monitoring Committee was still in place and I reported to them on a case by case basis as in stage one.

Due to the number of deaths in group 4, the Data Monitoring Committee was consulted and the study suspended for approximately six weeks. After a thorough review of the clinical and biological data, the Committee decreed that the deaths were not related to cooling and the study was completed as planned. 


\section{CHAPTER 3}

\section{METHODOLOGICAL ASPECTS}

\section{DESIGN OF THE STUDY}

The study design was that of a non-randomised, prospective pilot study. In the absence of data concerning hypothermia during ECMO a stepwise approach to both temperature reduction and length of cooling time was applied. This allowed careful scrutiny of both the infants and function of the ECMO circuit.

Since this was a pilot study, a power calculation of sample size was not performed. The results of the study were to be used to contribute to the power calculation for any subsequent randomised controlled trial.

The patients were not randomised to a particular temperature group but instead joined the groups in order. The next reduction in temperature only occurred after the successful completion of the previous stage.

\subsection{METHODS}

The study included twenty five neonates referred for ECMO at the Glenfield Hospital, Leicester between $1^{\text {st }}$ January 2000 and August 2001. 


\section{Entry Criteria}

The criteria for entry to the study were:

1. neonates greater than 33 weeks gestation, less than twenty eight days of age, birth weight between $2-5 \mathrm{Kg}$, suffering from severe but potentially reversible respiratory failure (defined as oxygenation index $\geq 440$, or arterial partial pressure of carbon dioxide $\left(\mathrm{PaCO}_{2}\right)$ greater than $12 \mathrm{kPa}$ for at least three hours).

2. less than ten days of high pressure ventilation

3. no contraindication for ECMO support (e.g. ventricular haemorrhage extending beyond the subependymal or choroidal region, presence of a major congenital or chromosomal anomaly, or severe encephalopathy).

Apart from the period of hypothermia, management of the ECMO circuit and patients was identical to the standard management policy. ${ }^{290}$

\section{ECMO Cannulation}

On admission to the Paediatric Intensive Care Unit, infants were assessed immediately by the consultant cardiothoracic surgeon in charge of ECMO. Either venoarterial (VA) or venovenous (VV) bypass was selected according to the infant's needs and surgeon's preference.

Patients were cannulated via the right internal jugular vein for drainage and the right common carotid for return in VA bypass, and the right internal jugular vein was cannulated with double-lumen cannula (12 or $15 \mathrm{Fr}$, Origen ${ }^{\circledR}$, Chalice Medical, Worksop, UK) for VV bypass. In the case of VA bypass, both vessels were ligated 
peripherally, and in the case of VV bypass semi-Seldinger technique was used for cannulation to allow patency of the right internal jugular vein.

\section{ECMO Circuit}

A standard ECMO circuit was used consisting of $1 / 4$ inch polyvinylchloride Tygon S-

65-HL tubing (Norton Performance Plastics, Acton, $\mathrm{OH}$ ), $0.8 \mathrm{~m}^{2}$ spiral wound silicone - membrane oxygenator (Model 0800) with in-line counter-current heat exchanger and collapsible assist reservoir (Avecor Cardiovascular, Plymouth, MN). A roller pump (Stockert; Sorin Biomedical; Saluggia, Italy) with Seabrook bladder box servo controller was used. The circuit was flushed with carbon dioxide and then filled with approximately $500 \mathrm{mls}$ of Ringers lactate solution and albumin. The clear prime was then displaced by one unit of citrated packed red cells to which calcium and heparin had been added. The $\mathrm{pH}$ was adjusted with sodium bicarbonate before use.

\section{Management of ECMO}

The ECMO circuit was managed for twenty four hours a day by specialist nurses trained in ECMO and capable of performing surveillance and emergency repairs to the circuit. As soon as the patients were stabilised on ECMO, ventilator settings were reduced gradually to "rest settings", i.e. peak pressure of $20 \mathrm{cmH}_{2} \mathrm{O}$, positive end expiratory pressure of $10 \mathrm{cmH}_{2} \mathrm{O}$, low rate (10 breaths/min) and low $\mathrm{FiO}_{2}$ of $30 \%$. Patients were sedated with morphine and midazolam but not paralysed. The activated clotting time (ACT) was monitored hourly using a Hemochron 401 analyser (International Technidyne Inc., Edison, NJ) and maintained between 160 and 200 
seconds by adjusting heparin dosage during ECMO. Blood flow rate and sweep gas flow through the oxygenator was adjusted to keep $\mathrm{PaO}_{2}$ 6-8 $\mathrm{kPa}$ or $\mathrm{SaO}_{2}$ (oxygen saturation in arterial blood) $>85 \%$ and $\mathrm{PaCO}_{2} 4-6 \mathrm{kPa}$ in VV bypass, $\mathrm{PvO}_{2}$ (partial pressure of oxygen in venous blood) $4-6 \mathrm{kPa}$ or $\mathrm{SvO}_{2}$ (oxygen saturation in venous blood) $>65 \%$ and $\mathrm{PvCO}_{2}$ (partial pressure of carbon dioxide in venous blood) $6-8 \mathrm{kPa}$ in VA bypass. Oxygenator function was checked by measuring pre and post oygenator blood gases. Haemoglobin concentrations were maintained above $14 \mathrm{~g} / \mathrm{dl}$ to maximise the oxygen carrying capacity and platelet counts were kept above $100,000 / \mathrm{mm}^{3}$ by transfusion. Prothrombin time, activated partial thromboplastin time and fibrinogen values were monitored and corrected by administering fresh frozen plasma and cryoprecipitate. Albumin values were maintained above $3.5 \mathrm{~g} / \mathrm{dl}$ by administering human albumin solution. Patients were diuresed to their dry weight. Patients were weaned from ECMO and decannulated when chest radiograph appearance and lung compliance improved, and adequate gas exchange without excessive ventilation (peak pressure $<30 \mathrm{cmH}_{2} \mathrm{O}, \mathrm{FiO}_{2}<60 \%$ ) could be demonstrated during a "trial off" ECMO. 


\subsection{THE ECMO CIRCUIT : INFANT RECEIVING VENO-VENOUS ECMO}

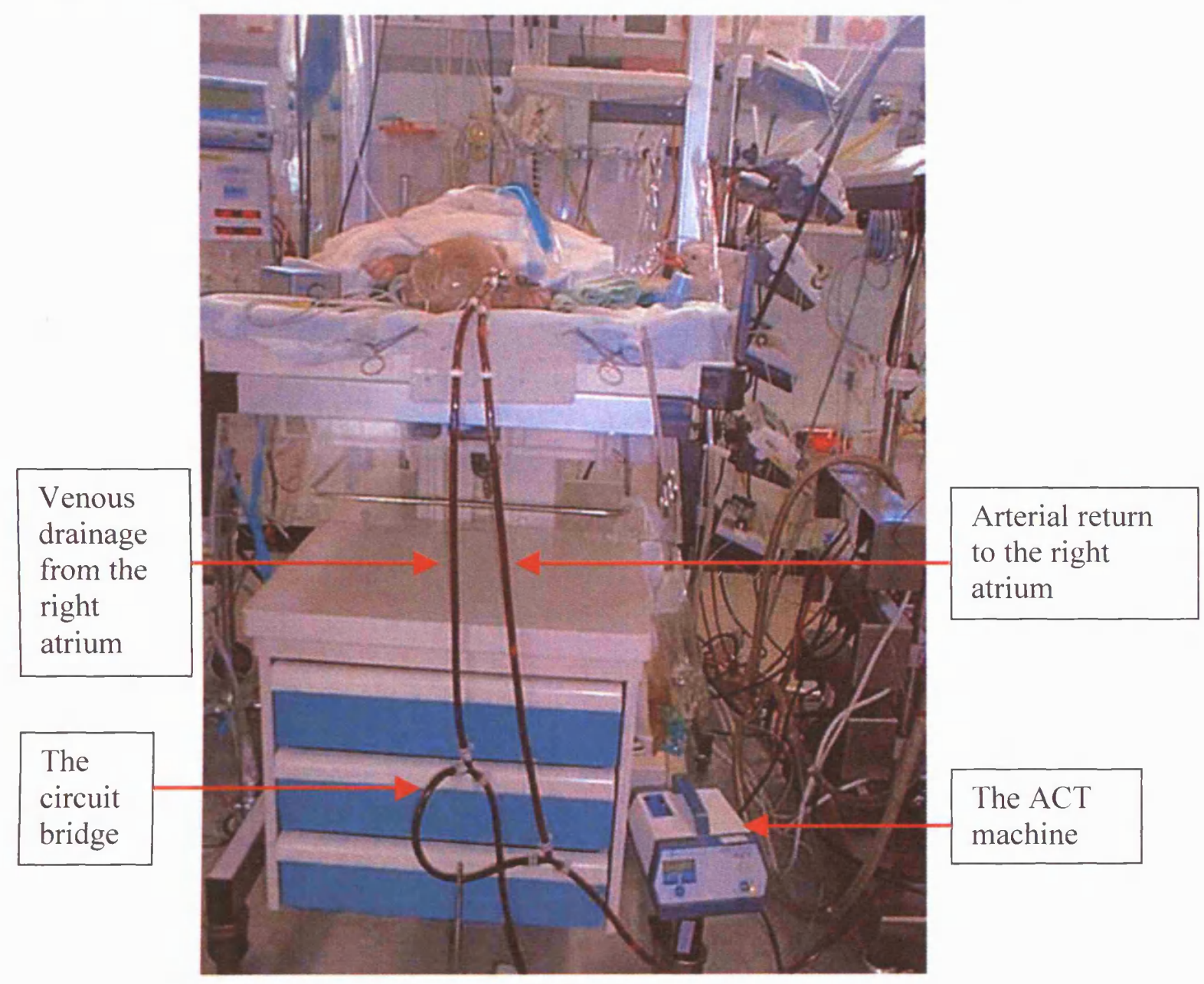

OVERVIEW OF NEONATAL ECMO

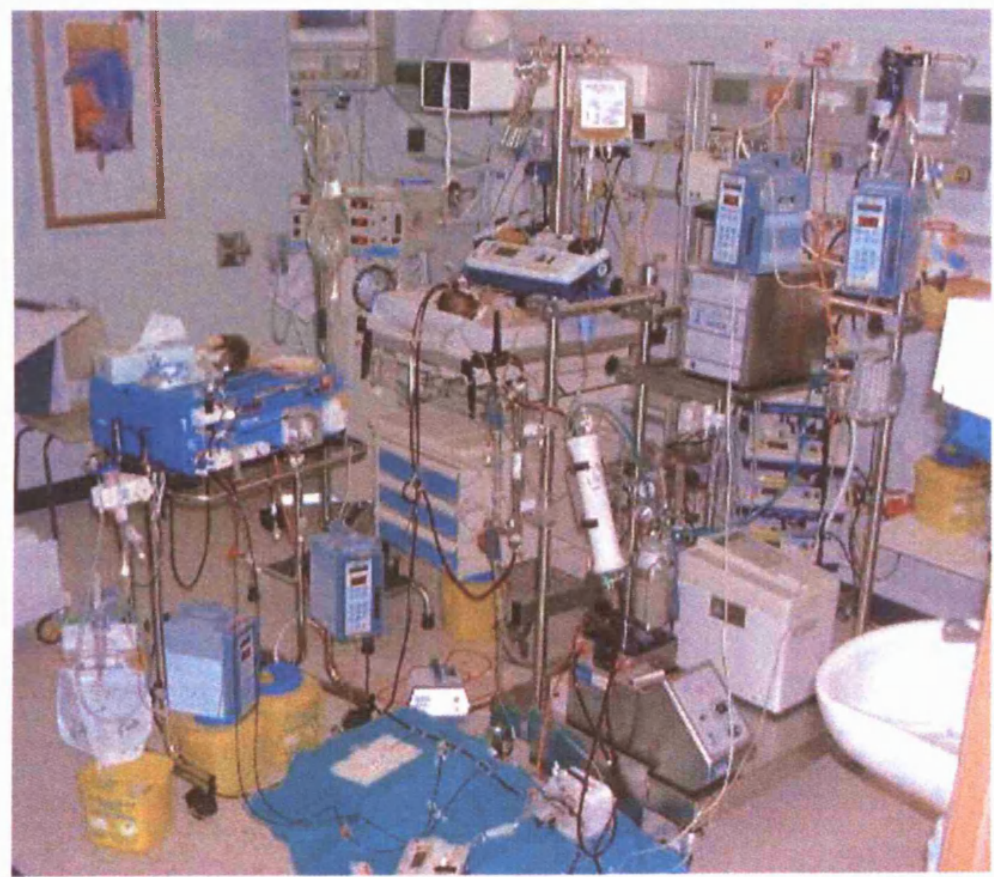


THE NEONATAL MEMBRANE OXYGENATOR

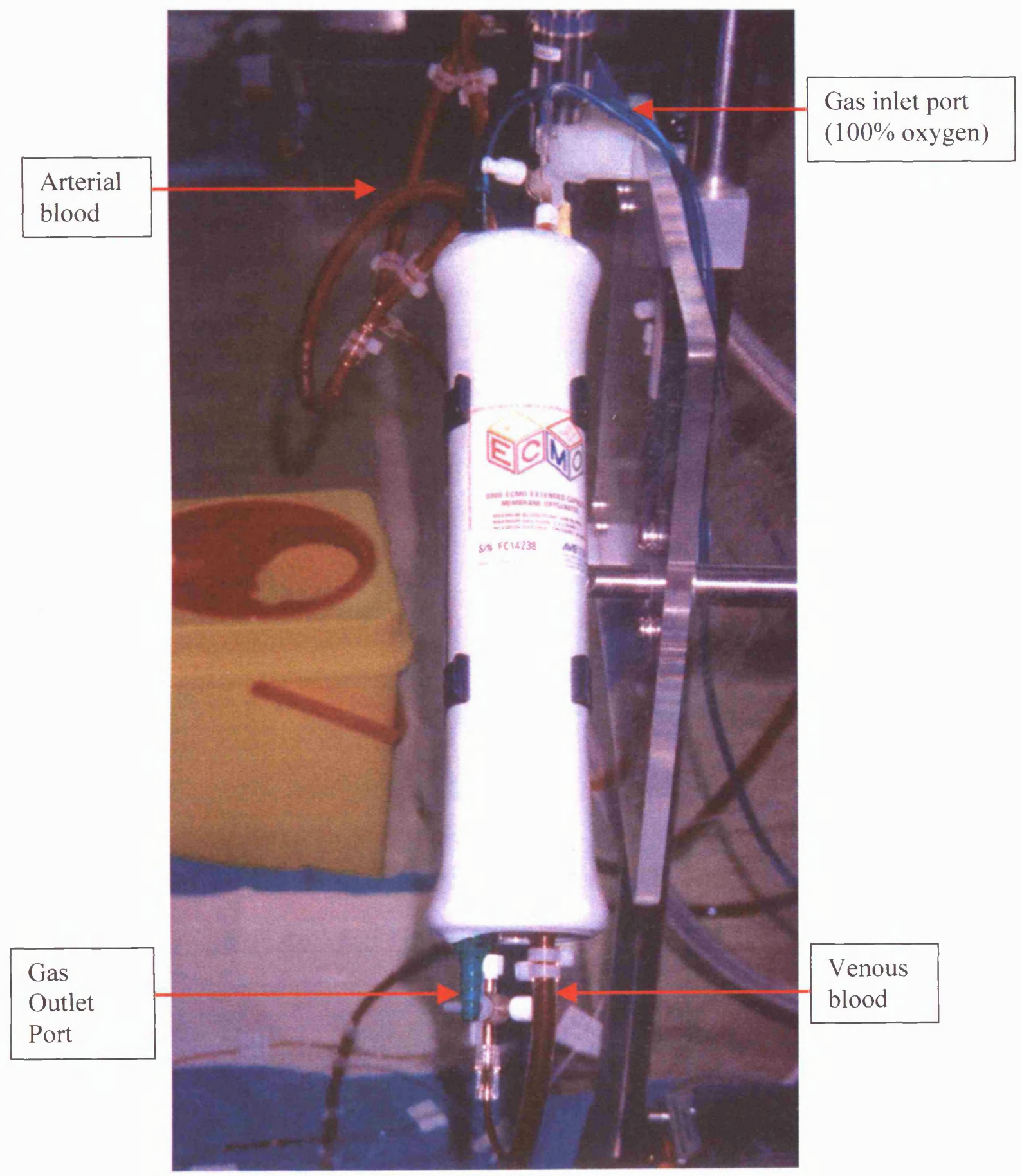




\section{THE STOCKERT ROLLER PUMP}

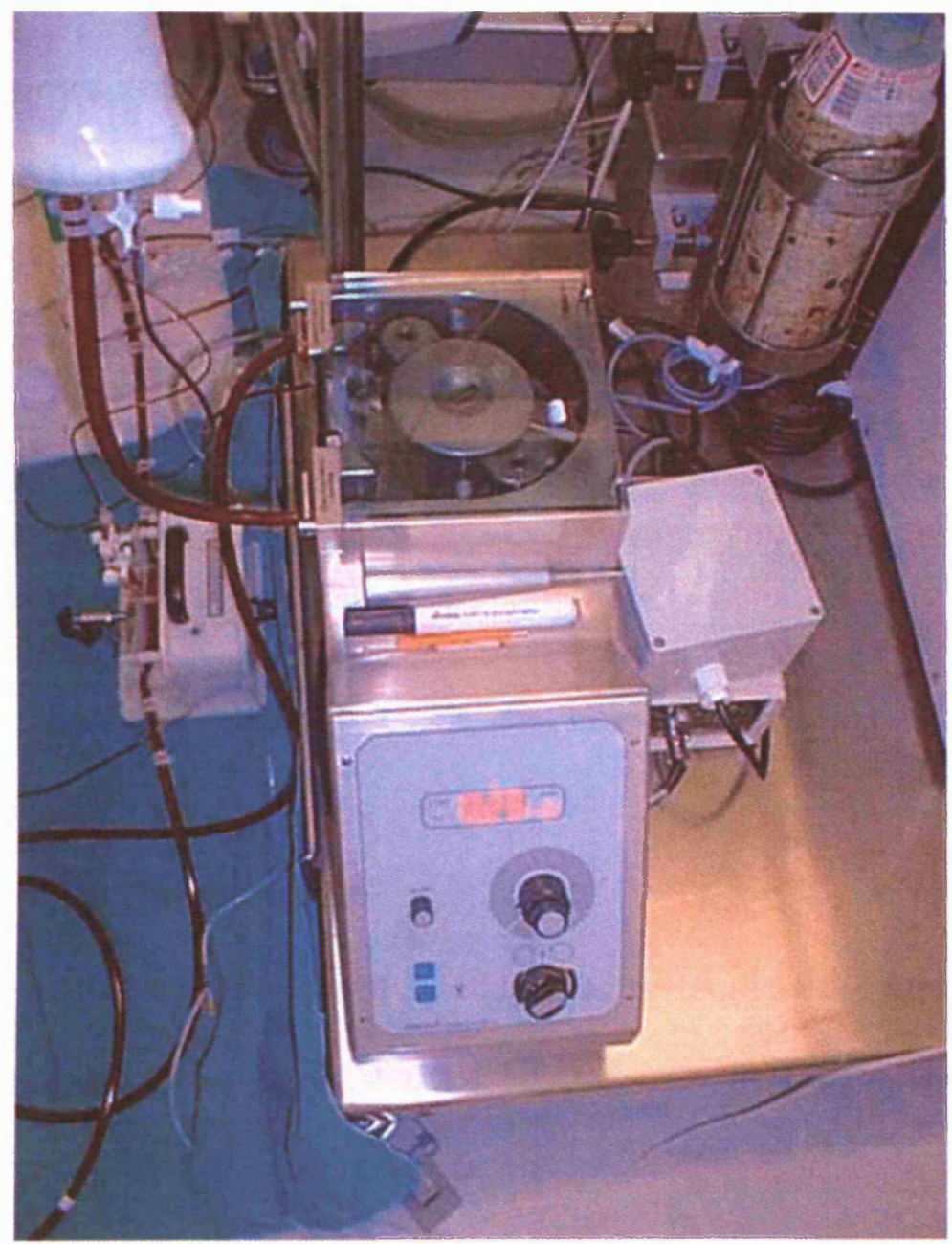

DOUBLE LUMEN NEONATAL ECMO CANNULA

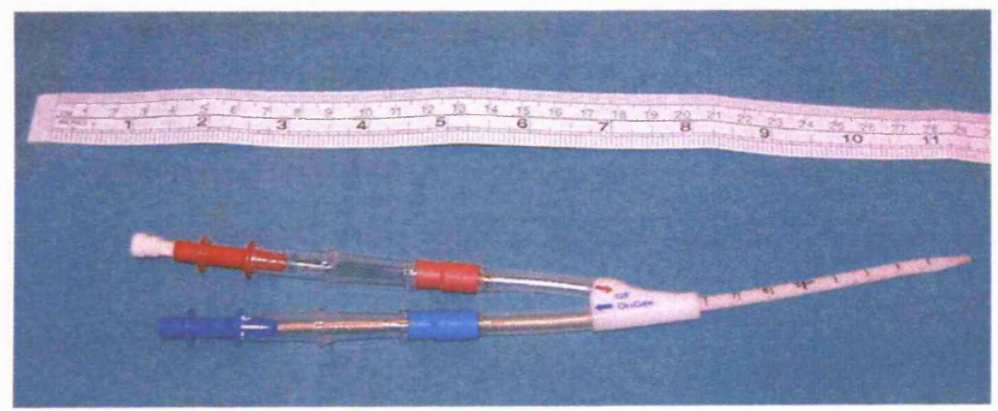




\subsection{STUDY GROUP}

Twenty five consecutive neonates (five groups $\mathrm{N}=5$ ) referred with severe respiratory failure for ECMO at the Glenfield Hospital, Leicester were enrolled in the study between January 2000 and August 2001. The infants were studied during the first five days of their ECMO run.

\section{Stage1: January 2000-January 2001}

Group 1: Five neonates maintained at $37^{\circ} \mathrm{C}$ core temperature throughout the first five days of ECMO (control group)

Group 2: Five neonates maintained at $36^{\circ} \mathrm{C}$ core temperature for the first 24 hours of $\mathrm{ECMO}$, then rewarmed to $37^{\circ} \mathrm{C}$ for the next four days

Group 3: Five neonates maintained at $35^{\circ} \mathrm{C}$ core temperature for the first 24 hours of ECMO, then rewarmed to $37^{0} \mathrm{Cfor}$ the next four days.

Group 4: Five neonates maintained at $34^{0} \mathrm{C}$ core temperature for the first 24 hours of ECMO, then rewarmed to $37^{0} \mathrm{C}$ for the next four days.

\section{Stage 2: June 2001 - August 2001}

Group 5: Five neonates maintained at $34^{0} \mathrm{C}$ core temperature for the first 48 hours of ECMO, then rewarmed to $37^{\circ} \mathrm{C}$ for the next three days. 


\subsection{HYPOTHERMIC TREATMENT}

Systemic hypothermia was commenced immediately following cannulation for

ECMO. The temperature of the blood within the extracorporeal circuit and hence the patient's core temperature can be controlled by adjustment of the water heater (Cincinnati SubZero heater, Cincinnati, OH) and the heat exchanger (Avecor Cardiovascular, Plymouth, MN) in the extracorporeal circuit. (see Figure 3.5) The heat exchanger consists of a narrow tubular device in which the blood flows through the inside of stainless steel conduits whilst warm water flows in a countercurrent manner on the outside.

The water heater temperature is typically set at $1^{0} \mathrm{C}$ above the desired body temperature in anticipation that heat is lost from the blood during perfusion through the tubing connected to the patient.

The water heater setting was adjusted to maintain the desired rectal temperature for the first 24 or 48 hours of ECMO.

Heat exchanger failure is rare, occurring in only $1 \%$ of neonatal extracorporeal life support cases. ${ }^{291}$ The most common complication is leak of water from the connectors at the top and bottom of the heat exchange device.

\section{Rewarming}

At the end of the cooling period, the patients were rewarmed slowly at a rate of no more than $0.5^{\circ} \mathrm{C}$ per hour. Rewarming was achieved by adjustment of the water bath temperature and the use of an overhead radiant heater. 
The rationale for rewarming slowly was because of concerns of disturbance in the potassium balance if patients were rewarmed rapidly. Hypokalaemia has been described during hypothermia ${ }^{280}$ and is thought to result from intracellular potassium redistribution. ${ }^{279}$ In adult rats, Sprung et al ${ }^{279}$ showed a significant decrease in the potassium concentration during cooling to $30^{\circ} \mathrm{C}$. There was spontaneous normalisation of serum potassium during rewarming to normothemia. In a second experiment, hypokalaemia during hypothermia was corrected with potassium chloride. In these animals, the serum potassium was significantly higher in the normothermic rats and ventricular ectopics and atrio-ventricular blocks were present during the period of rewarming. It may be that during emergence from hypothermia, recovery of the mechanism that physiologically counteracts hyperkalaemia (energy dependent ionic pump) may lag behind the actual rise in body temperature. My theoretical concern was that by rapidly rewarming the patients, the slow potassium transport mechanism might be overburdened resulting in hyperkalaemia due to endogenous shifts of potassium load. 


\subsection{THE HEAT EXCHANGER}

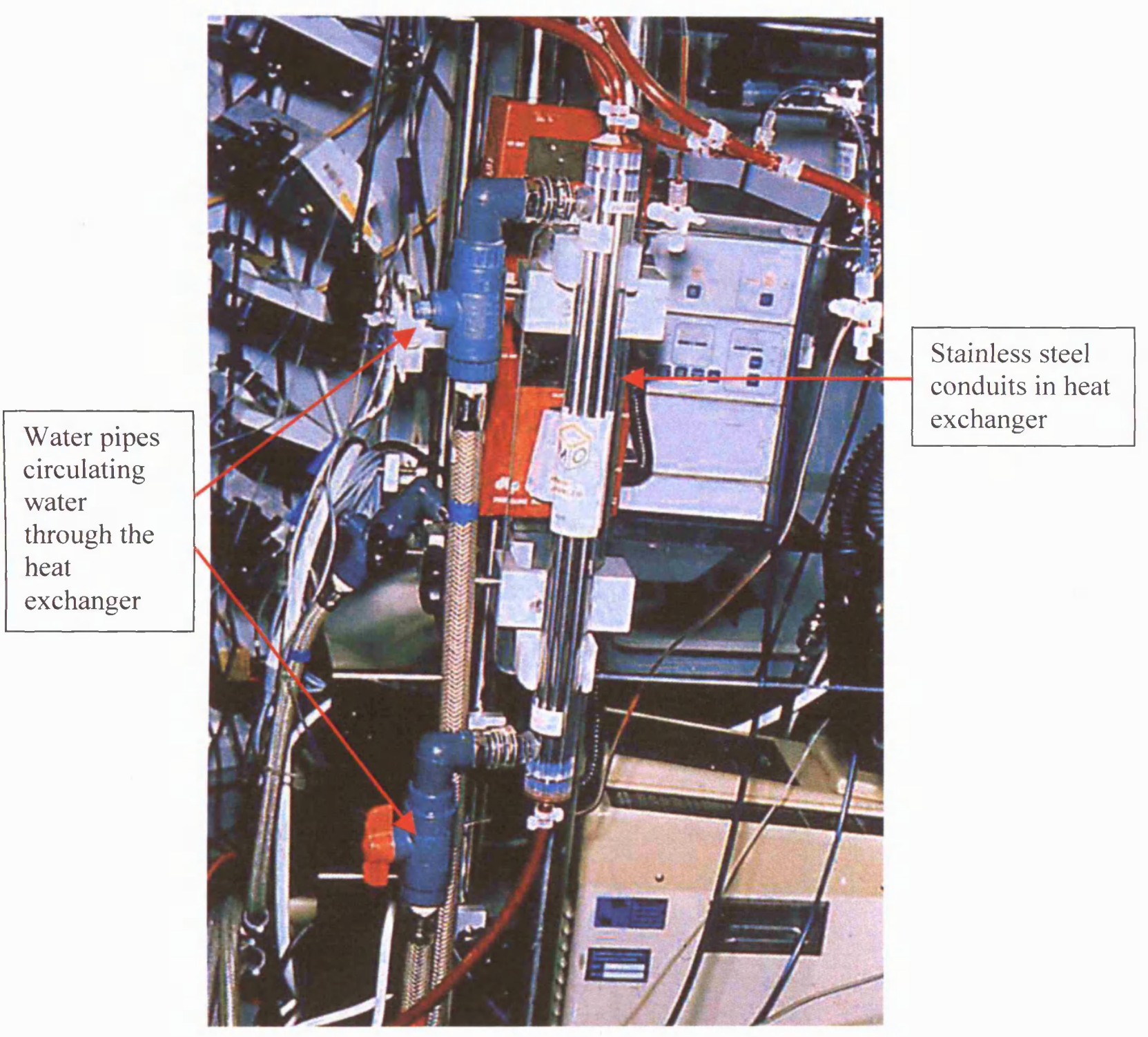


THE CINCINNATI SUB-ZERO WATER HEATER

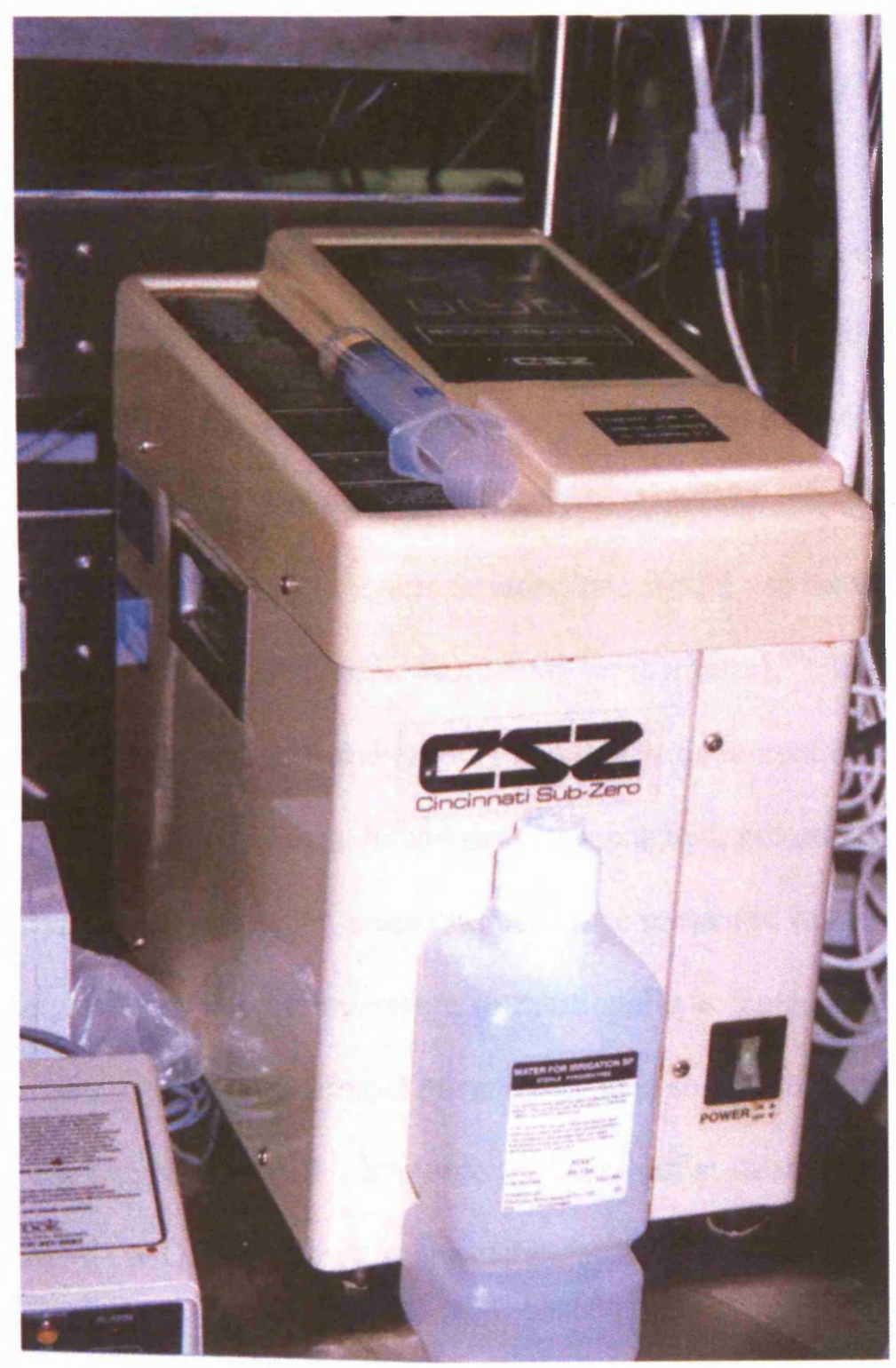




\subsection{MEASUREMENT OF THE CORE TEMPERATURE}

The rectal temperature was continuously monitored and taken to represent the baby's core temperature.

In this study, accurate measurement of the core temperature was essential. However, in choosing the method of core temperature measurement, consideration was given to the practical implications of temperature measurement on ECMO in addition to ensuring a precise repeatable measure of core temperature.

Ideally, for clinical decisions on temperature, one would use the temperature of the blood perfusing the hypothalamus (core body temperature). ${ }^{292}$ However, this site is not accessible so oral, rectal and axillary and tympanic temperatures have been used particularly in awake patients. In addition, oesophageal, pulmonary artery (PA), nasopharyngeal, and bladder temperatures can be measured in anaesthetised patients. Pulmonary arterial blood temperature is traditionally accepted as the reference measurement of core body temperature; it has been shown to be only $0.18^{0} \mathrm{C}$ lower than the temperature in the high internal jugular vein at steady state. ${ }^{293}$ In the context of neonatal ECMO, pulmonary artery catheters are not routinely sited therefore pulmonary artery temperature measurement was not feasible for this study. Nasopharyngeal temperature monitoring was not possible in view of the risk of haemorrhage in a heparinised patient. The axillary temperature has been repeatedly shown to be the least accurate measurement of the core temperature and not suitable for this study.

Aural infrared thermometers have gained widespread use particularly in paediatric practice since they are less invasive and give a rapid, convenient temperature measurement. However, from the published data there is some doubt over the 
accuracy of aural temperature measurement compared to that measured by other methods including rectal ${ }^{294}$ and pulmonary artery thermometers. ${ }^{295}$ In a systematic review reported by Craig et $\mathrm{al}^{296}$ comparing ear thermometry with rectal thermometry, the mean difference between the ear and rectal temperature measurements were small. However, the wide limits of agreement imply that the ear temperature was not a good approximation of rectal temperature, even when the ear thermometer was used in rectal mode. "Rectal mode" means that some tympanic thermometers have built-in off sets that attempt to estimate the core, oral or rectal temperature from the actual tympanic temperature hence they can be set to measure in "rectal mode". Other studies have reported close agreement between ear and rectal temperature measurements in infants. ${ }^{297}$ However, in this study comparing temperatures obtained from 140 infants and toddlers, it was not clear whether the tympanic thermometer was in core, "rectal equivalent" or "oral equivalent" mode which makes interpretation of the results more difficult. As well as the concerns over its accuracy, another disadvantage in using tympanic thermometry for this study, was that only point measurements rather than continuous temperature measurement would have been obtained.

Tympanic membrane thermocouple measurements have been used in adult studies as an accurate reference for tympanic thermometers. ${ }^{298}$ There are no validated studies of the use of tympanic membrane thermocouples in neonatal intensive care and they are not available for routine clinical use.

The urinary bladder temperature has been reported as a reliable method of core temperature measurement in adult patients. ${ }^{299-300}$ Once again, there are no validated studies of the use of urinary bladder temperature in neonates. Also, there is some evidence that the urinary bladder temperature may be affected by a decrease urine 
volume due to an increase in the production of antidiuretic hormone that accompanies hypotension, hypovolaemia and hypoxia. ${ }^{301}$ This situation may arise when the patient is first cannulated and stabilised during the first hours of ECMO. In addition, ECMO patients are diuresed to their dry weight which may further affect the accuracy of the urinary bladder temperature.

For many years, the rectal temperature has been used in clinical practice as the "gold standard" for core temperature measurement. Although during rapid rewarming from deep hypothermia on cardiac bypass, there was reported to be a lag in the change in rectal temperature, ${ }^{302}$ the rectal temperature has been shown to predict accurately the core temperature measured at the pulmonary artery. ${ }^{303}$ There is long standing clinical experience of measuring rectal temperatures in neonates. Clinical decisions are guided by rectal thermometry ${ }^{304-305}$ and clinicians regularly use the rectal site to check a temperature measured elsewhere. ${ }^{306}$

Using continuous rectal thermometry in this study meant that the core temperature could be measured accurately without disrupting routine patient care. In addition, it was possible to ensure that the patients were maintained at a steady temperature state once the target temperature was reached and the rate of cooling and rewarming could be observe.

\subsection{MONITORING AND BLOOD SAMPLE COLLECTION}

Rectal and abdominal skin temperatures were monitored continuously. Arterial blood pressure, ECG, respiratory rate and pulse oxymetry were shown continuously on the monitor screen (Hewlett Packard HP Component Monitoring System Model 66S) according to routine practice in the ECMO centre. 
Routine samples including blood gas, full blood count, electrolytes, liver function profile, coagulation studies and C-reactive protein were drawn prior to ECMO cannulation ( $\mathrm{t}=0$ hours). In groups $1-4$, following ECMO cannulation further routine samples were drawn from the arterial line at timed intervals of 2, 12, 24, 36, 48, 72, 96 and 120 hours. In group 5 (48 hour cooling), samples were drawn at the same time points except at 60 instead of 36 hours.

Blood samples for assays not available in the routine laboratory were also drawn at the same time intervals. These were centrifuged at $3000 \mathrm{RPM}$ at $4^{0} \mathrm{C}$ for 15 minutes and stored at $-70^{\circ} \mathrm{C}$ until further analysis.

Oxygenator resistance was calculated at 2 hours, 12 hours and thereafter 24 hourly using the following formula: -

(Pre -oxygenator pressure minus post-oxygenator pressure $(\mathrm{mmHg}))$

Blood flow rate $(\mathrm{ml} / \mathrm{min})$

Heparin, colloid and platelet requirements were recorded 12 to 24 hourly. Investigations for sepsis were conducted and repeated if clinically indicated. In addition the QTc interval was measured using both standard 12 lead ECG's and a digital 24 hour Holter monitor. Finally the amplitude-integrated electroencephalogram was measured continuously using a cerebral function monitor for the first 120 hours of ECMO. 


\section{CHAPTER 4}

\section{DEMOGRAPHIC DATA AND CORE TEMPERATURE CONTROL}

\section{DEMOGRAPHIC DATA}

Thirty one patients were recruited but six were excluded from the study for the following reasons; two patients required surgery within the study period which resulted in re-cooling to $35.5^{\circ} \mathrm{C}$ for greater than three hours; one infant was excluded due to an error in following the study protocol where blood sampling was discontinued following decannulation from ECMO at 72 hours; two patients developed a sinus tachycardia of greater than 180 beats per minute and a clinical decision was made to cool the patients at 72 hours of ECMO in an attempt to reduce the heart rate. Finally, in one patient, ECMO support was withdrawn after 12 hours because of an inoperable cardiac lesion.

The clinical characteristics of the twenty five infants included in the study are summarised in table 1. No significant difference was found between the five groups in terms of pre-ECMO demographic data. All patients had severe respiratory failure unresponsive to conventional therapy. The primary diagnoses are shown in table 1. Although there were two patients with rare diagnoses, the disease pattern reflected those normally seen at our institution.

In those patients not surviving to discharge (table 2) the cause of death was as follows. In group 2, intensive care was withdrawn in patient 9 due to inoperable total anomalous pulmonary venous drainage (TAPVD). In group 4, patient 16 had an inborn error of metabolism (myophosphorylase deficiency), patient 17 suffered a large posterior fossa cerebral haemorrhage on day 11 of ECMO and patient 20 developed 
multiorgan failure secondary to presumed pertussis sepsis and died on day 8 of ECMO.

The overall survival rate to discharge was $84 \%$, which corresponds to the expected ECMO survival rate for our institution. We reviewed the three deaths in group four, with the assistance of the data monitoring committee, before continuing with the study. We could find no clinical or statistical evidence to suggest that the deaths were related to cooling.

Details of ECMO related complications are shown in table 2. The majority of infants $(80 \%)$ were managed using veno-venous ECMO. All patients with congenital diaphragmatic hernia $(\mathrm{CDH})$ were repaired on ECMO following stabilisation. No significant difference was found between groups for length of time on ECMO (Kruskal-Wallis chi-squared $=3.1, \mathrm{df}=4, \mathrm{p}=0.5$ ). No circuit was exchanged during the five day study period. However, patient 16 (group 4) was converted from venovenous to veno-arterial ECMO due to cardiovascular instability on day 2 of ECMO.

\subsection{CORE TEMPERATURE}

There was no significant difference in the temperature between groups prior to starting cooling. Cooling was initiated immediately following cannulation for ECMO. Using the extracorporeal circuit accurate control of the core temperature was possible (as illustrated in figure 1).

Cooling was achieved in groups $2-5$ by a median of 4 hours (range 2-7 hours). At the end of cooling the patients were rewarmed to $37^{\circ} \mathrm{C}$ at a rate of $0.5^{0} \mathrm{C}$ per hour. In group 5, the median time to achieve rewarming was 64 hours from commencing ECMO (range 55-69 hours). 


\subsection{TABLES AND FIGURES}

Table 1 Summary of Demographic Data for Study Groups ( $\mathrm{n}=5$ per group) Median values (Range)

\begin{tabular}{|c|c|c|c|c|c|c|c|}
\hline & $\begin{array}{l}\text { Group } 1 \\
\left(37^{0} \mathrm{C}\right)\end{array}$ & $\begin{array}{l}\text { Group } 2 \\
\left(36^{0} \mathrm{C} \text { for }\right. \\
24 \text { hours) }\end{array}$ & $\begin{array}{l}\text { Group } 3 \\
\left(35^{\circ} \text { Cfor }\right. \\
24 \text { hours }) \\
\end{array}$ & $\begin{array}{l}\text { Group } 4 \\
\left(34^{0} \text { Cfor }\right. \\
24 \text { hours }) \\
\end{array}$ & $\begin{array}{l}\text { Group } 5 \\
\left(34^{0} \mathrm{C} \text { for }\right. \\
48 \text { hours })\end{array}$ & Chi-squared* & $\mathrm{p}$ \\
\hline $\begin{array}{l}\text { Gestation in } \\
\text { Weeks }\end{array}$ & $\begin{array}{c}40 \\
(33-40)\end{array}$ & $\begin{array}{c}40 \\
(38-40)\end{array}$ & $\begin{array}{c}40 \\
(38-41)\end{array}$ & $\begin{array}{c}38 \\
(37-40)\end{array}$ & $\begin{array}{l}40 \\
(39-41)\end{array}$ & 5.5 & 0.2 \\
\hline $\begin{array}{l}\text { Age at ECMO } \\
\text { In hours }\end{array}$ & $\begin{array}{c}24 \\
(22-37)\end{array}$ & $\begin{array}{c}30 \\
(25-432)\end{array}$ & $\begin{array}{c}24 \\
(16-94)\end{array}$ & $\begin{array}{c}50 \\
(6-384)\end{array}$ & $\begin{array}{c}22 \\
(12-26)\end{array}$ & 8.2 & 0.1 \\
\hline $\begin{array}{l}\text { Birth Weight } \\
\quad(\mathrm{Kg})\end{array}$ & $\begin{array}{c}2.6 \\
(2.4-3.7)\end{array}$ & $\begin{array}{c}3.7 \\
(3.2-4.3)\end{array}$ & $\begin{array}{c}3.4 \\
(2.8-4.7)\end{array}$ & $\begin{array}{c}3.7 \\
(2.7-4.4)\end{array}$ & $\begin{array}{c}3.4 \\
(3.1-4.3)\end{array}$ & 4.2 & 0.4 \\
\hline Males & 4 & 5 & 3 & 3 & 4 & & \\
\hline $\begin{array}{l}\text { Apgar Score } \\
1 \text { minute }\end{array}$ & $\begin{array}{c}7 \\
(5-8)\end{array}$ & $\begin{array}{c}6 \\
(1-9)\end{array}$ & $\begin{array}{c}7 \\
(4-10)\end{array}$ & $\begin{array}{c}9 \\
(6-10)\end{array}$ & $\begin{array}{c}5 \\
(5-6)\end{array}$ & 6.2 & 0.2 \\
\hline $\begin{array}{l}\text { Apgar Score } \\
5 \text { minutes }\end{array}$ & $\begin{array}{c}9 \\
(7-10)\end{array}$ & $\begin{array}{c}8 \\
(5-10)\end{array}$ & $\begin{array}{c}9 \\
(8-10)\end{array}$ & $\begin{array}{l}10 \\
(9-10)\end{array}$ & $\begin{array}{c}8 \\
(8-9)\end{array}$ & 5.0 & 0.3 \\
\hline Ph Pre-ECMO & $\begin{array}{c}7.2 \\
(7.0-7.5)\end{array}$ & $\begin{array}{c}7.3 \\
(7.0-7.4)\end{array}$ & $\begin{array}{c}7.3 \\
(7.0-7.7)\end{array}$ & $\begin{array}{c}7.3 \\
(7.0-7.3)\end{array}$ & $\begin{array}{c}7.2 \\
(7.0-7.4)\end{array}$ & 0.76 & 0.8 \\
\hline $\begin{array}{l}\text { Oxygenation } \\
\text { Index }\end{array}$ & $\begin{array}{c}50 \\
(35-70)\end{array}$ & $\begin{array}{c}48 \\
(27-89)\end{array}$ & $\begin{array}{c}40 \\
(28-90)\end{array}$ & $\begin{array}{c}31 \\
(20-52)\end{array}$ & $\begin{array}{c}40 \\
(31-55)\end{array}$ & 3.2 & 0.5 \\
\hline \multicolumn{8}{|c|}{$\underline{\text { Primary Diagnoses }}$} \\
\hline $\begin{array}{l}\text { MAS/PPHN } \\
\text { Sepsis } \\
\text { RDS }\end{array}$ & $\begin{array}{l}3 \\
1 \\
1\end{array}$ & 3 & $\begin{array}{l}4 \\
1\end{array}$ & $\begin{array}{l}2 \\
1\end{array}$ & $\begin{array}{l}4 \\
1\end{array}$ & & \\
\hline $\begin{array}{l}\text { Congenital } \\
\text { Diaphragmatic } \\
\text { Hernia (CDH) } \\
\text { TAPVD } \dagger \\
\text { Inborn Error of } \\
\text { Metabolism }+\end{array}$ & & 1 & & 1 & & & \\
\hline
\end{tabular}

*Comparison of five groups by Kruskal-Wallis test $(\mathrm{df}=4)$

$\uparrow$ Total anomalous pulmonary venous drainage

† Myophosphorylase deficiency. (Possible Infantile $\mathrm{M}^{\mathrm{C}}$ Ardles type disorder) 
Table 2 Progress on ECMO and Complications Reported during the Study

\begin{tabular}{|c|c|c|c|c|c|}
\hline $\begin{array}{l}\text { Number of } \\
\text { Patients }\end{array}$ & $\begin{array}{r}\text { Group } 1 \\
\left(37^{0} \mathrm{C}\right)\end{array}$ & $\begin{array}{l}\text { Group } 2 \\
\left(36^{0} \text { Cfor }\right. \\
24 \text { hours })\end{array}$ & $\begin{array}{l}\text { Group } 3 \\
\left(35^{\circ} \mathrm{C} \text { for }\right. \\
24 \text { hours })\end{array}$ & $\begin{array}{l}\text { Group } 4 \\
\left(34^{0} \text { Cfor }\right. \\
24 \text { hours })\end{array}$ & $\begin{array}{l}\text { Group } 5 \\
\left(34^{0} \mathrm{C} \text { for }\right. \\
48 \text { hours })\end{array}$ \\
\hline $\begin{array}{l}\text { Venovenous } \\
\text { Cannulation }\end{array}$ & 4 & 3 & 5 & 3 & 5 \\
\hline $\begin{array}{l}\text { Median (range) time } \\
\text { on ECMO in hours }\end{array}$ & $\begin{array}{c}80 \\
(68-176)\end{array}$ & $\begin{array}{c}128 \\
(60-451)\end{array}$ & $\begin{array}{c}70 \\
(43-122)\end{array}$ & $\begin{array}{c}97 \\
(60-218)\end{array}$ & $\begin{array}{c}96 \\
(62-164)\end{array}$ \\
\hline Number of Deaths & 0 & 1 & 0 & 3 & 0 \\
\hline Bleeding & 0 & $1^{*}$ & 0 & 0 & 0 \\
\hline $\begin{array}{l}\text { Oxygenator } \\
\text { Failure }\end{array}$ & 0 & 0 & 0 & 0 & 0 \\
\hline Clots in circuit $\uparrow$ & 5 & 5 & 1 & 3 & 3 \\
\hline $\begin{array}{l}\text { Definite } \\
\text { Seizures }\end{array}$ & 0 & 1 & 0 & 1 & 0 \\
\hline $\begin{array}{l}\text { Possible } \\
\text { Seizures }\end{array}$ & 0 & 1 & 0 & 1 & $2 \%$ \\
\hline
\end{tabular}

* Bleeding around cannula site secondary to heparin bolus given too early during cannulation. Bleeding resolved by hour four of ECMO.

$\uparrow$ All reported clots were small and mainly in the ECMO circuit bladder.

\$ One episode of abnormal movement in both patients within first 12 hours of ECMO. Phenobarbitone stopped after 24 hours treatment. 
Figure 1 Rectal Temperature of Study Groups

Group 1 (Control Group) $37^{\circ} \mathrm{C}$

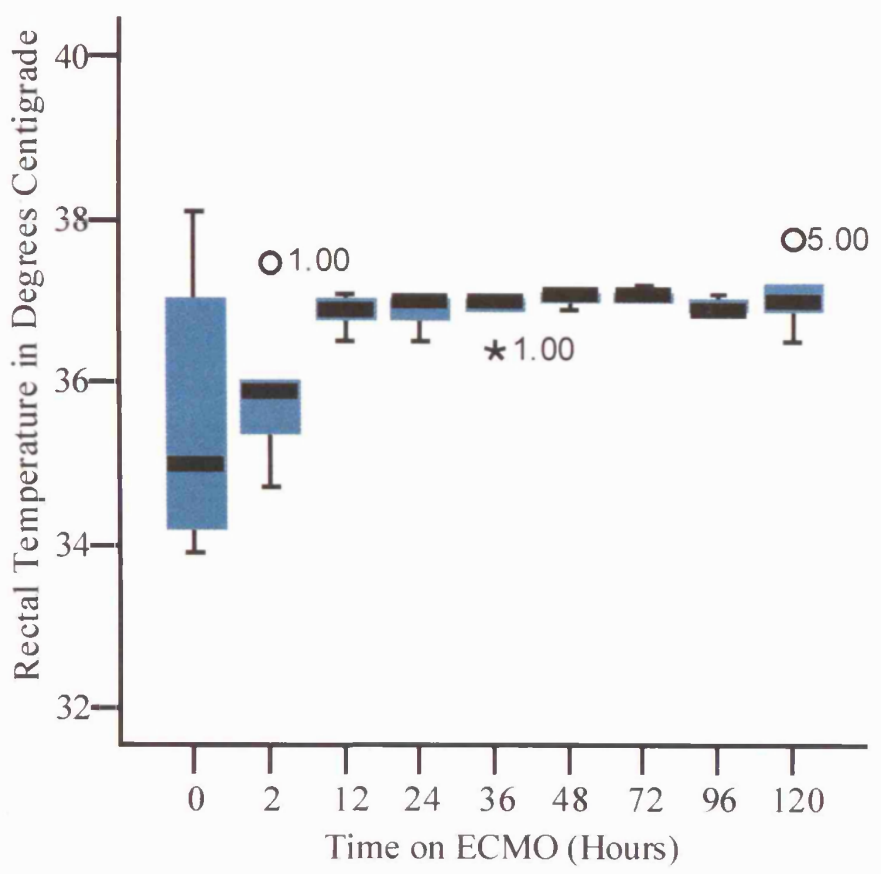

Group 2: Cooled to $36^{\circ} \mathrm{C}$ for 24 hours

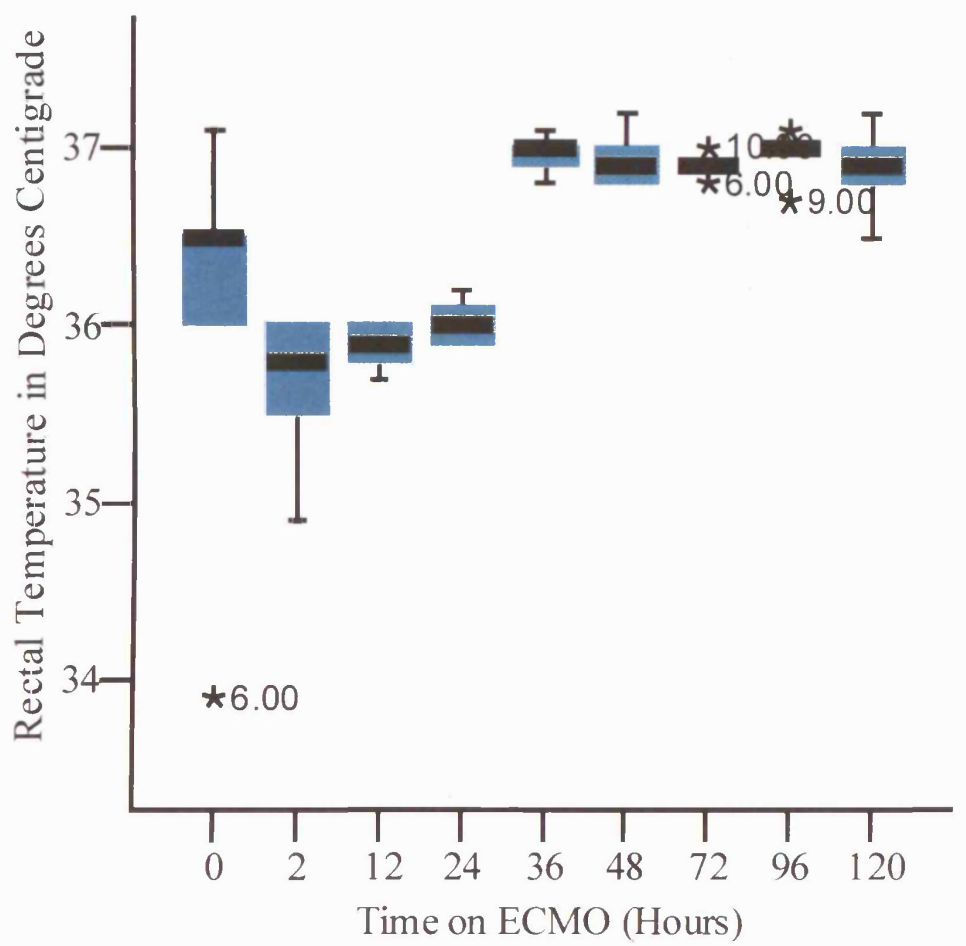



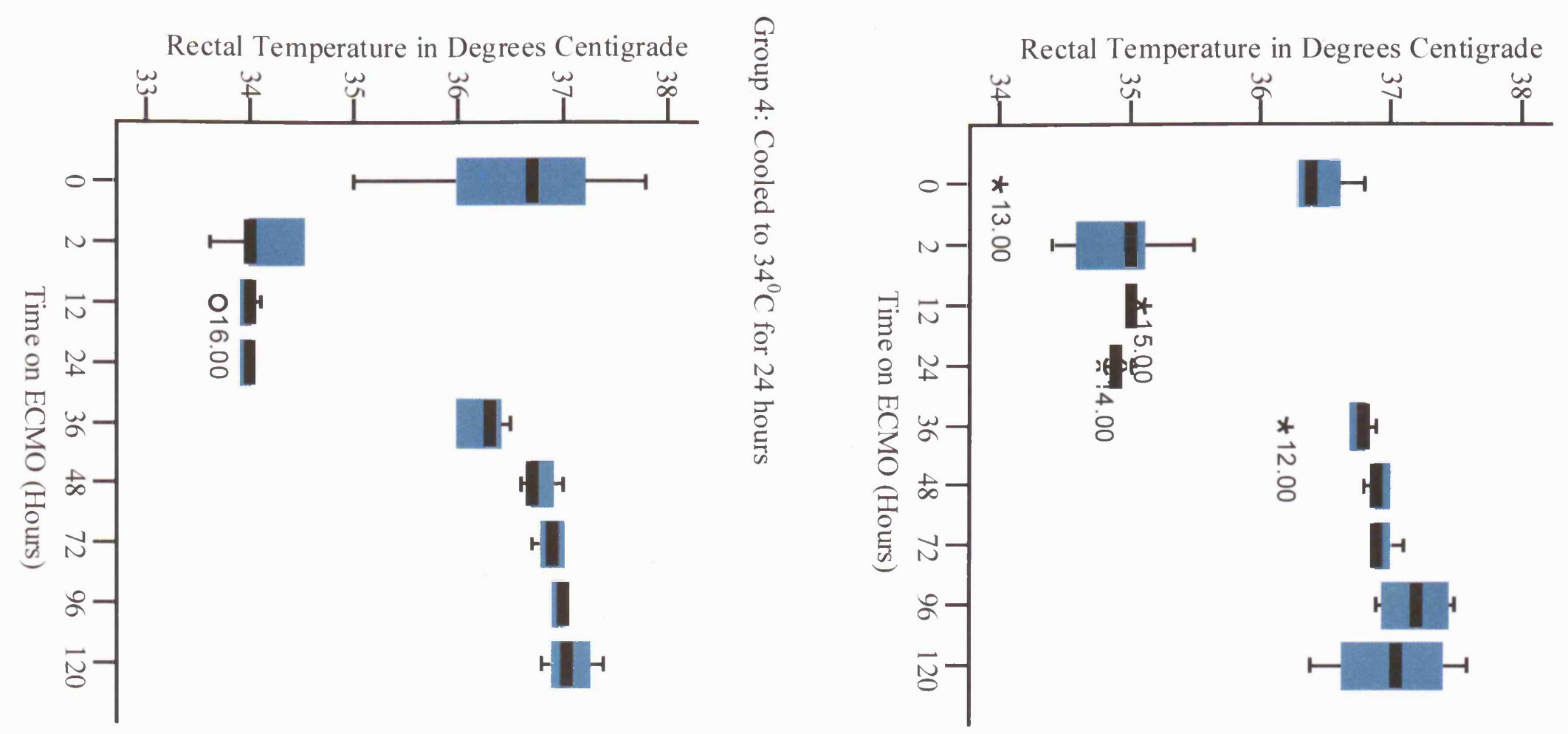
Group 5: Cooled to $34^{0} \mathrm{C}$ for 48 hours

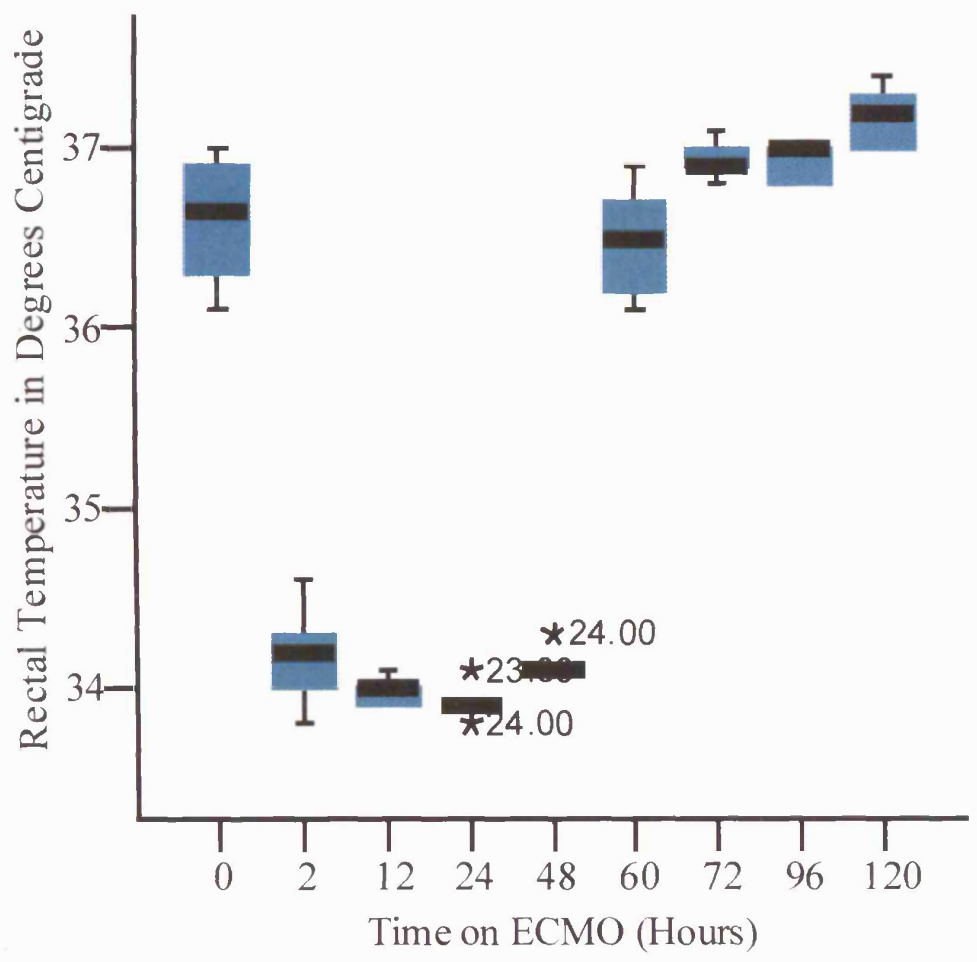

Figure 1: Illustrates the rectal temperature for each group during the study period. For each group the median and interquartile range is displayed for rectal temperature at time points $0-120$ hours. Using the extracorporeal circuit, accurate temperature control was possible during cooling and rewarming. 


\section{CHAPTER 5}

\section{THE EFFECT OF TEMPERATURE ON THE QTC INTERVAL}

\section{INTRODUCTION}

In adults, hypothermia is associated with a number of well documented changes on the electrocardiogram including sinus bradycardia, widening of the PR interval, Osborn J waves and prolongation of the QT interval, ${ }^{273,307}$ which may be a precursor to ventricular arrhythmias including the potentially lethal torsade de pointes. Previous authors have reported prolongation of the QT interval during moderate accidental hypothermia and induced severe progressive hypothermia in adult patients. ${ }^{274}$ However, few human studies have documented the effects of prolonged mild hypothermia on the QT interval. In a study of three poikilothermic adults, Mackenzie et $\mathrm{al}^{276}$ reported widening of the QT interval during 72 hours of steady state mild hypothermia (rectal temperature $33.9^{\circ} \mathrm{C}$ ) and atrioventricular conduction abnormalities on the ECG.

Recent interest in mild hypothermia as a neuroprotective strategy following hypoxicischaemic cerebral injury and ECMO therapy has raised the question of the potential adverse cardiovascular effects of mild cooling including cardiac arrhythmias. ${ }^{275}$ No systematic study has been published, but Gunn et $\mathrm{al}^{277}$ reported a prolonged QT interval of 570 milliseconds in one infant cooled to a rectal temperature of $34.4^{0} \mathrm{C}$ as a treatment for perinatal asphyxia.

As part of the pilot study I investigated the effect of mild induced hypothermia on the QTc interval over a range of temperatures from $34^{\circ} \mathrm{C}$ to $37^{\circ} \mathrm{C}$. I measured the QT and 
QTc interval using a 24 hour digital monitor and a standard 12 lead ECG's for validation of the automated values.

This part of the pilot study addressed the questions:

1) What is the relationship between rectal temperature and QTc?

a) at steady state and b) during changes in the rectal temperature induced by rewarming

2) Are any changes related to alterations in blood chemistry or clinical variables?

\subsection{METHODS}

The study group included the twenty five patients described in chapters 3 and 4 . Due to technical difficulties in obtaining the twenty four hour ECG recordings, two additional patients were recruited at the completion of stage one i.e. on completion of group $4\left(34^{0} \mathrm{C}\right.$ for 24 hours). These additional patients were maintained at $37^{\circ} \mathrm{C}$ for the first five days of their ECMO run and included as part of the control group. The entry criteria and ECMO management described in chapter 3 was identical for these two patients.

\section{Study Group}

Twenty seven neonates (group $1 \mathrm{~N}=7$ groups $2-5 \mathrm{~N}=5$ ) referred with severe respiratory failure for ECMO at the Glenfield Hospital, Leicester were enrolled in the study between $1^{\text {st }}$ January 2001 - August 2002 . The infants were studied during the first five days of their ECMO run. 
Group 1: Seven neonates maintained at $37^{\circ} \mathrm{C}$ core temperature throughout the first five days of ECMO (control group)

Group 2: Five neonates maintained at $36^{\circ} \mathrm{C}$ core temperature for the first 24 hours of $\mathrm{ECMO}$, then rewarmed to $37^{0} \mathrm{C}$ for the next four days

Group 3: Five neonates maintained at $35^{\circ} \mathrm{C}$ core temperature for the first 24 hours of ECMO, then rewarmed to $37^{\circ} \mathrm{C}$ for the next four days.

Group 4: Five neonates maintained at $34^{\circ} \mathrm{C}$ core temperature for the first 24 hours of ECMO, then rewarmed to $37^{0} \mathrm{C}$ for the next four days.

Group 5: Five neonates maintained at $34^{\circ} \mathrm{C}$ core temperature for the first 48 hours of ECMO, then rewarmed to $37^{\circ} \mathrm{C}$ for the next three days.

\section{Hypothermic Treatment}

The infants were cooled and rewarmed by adjusting the temperature of the water bath in the extracorporeal circuit as described in chapter 3.

\section{Monitoring and Blood Sample Collection}

Rectal and abdominal skin temperatures were monitored continuously. Arterial blood pressure, ECG, respiratory rate and pulse oxymetry were shown continuously on the monitor screen (Hewlett Packard HP Component Monitoring System Model 66S) 
according to routine practice.

Routine electrolyte samples (including calcium and magnesium), were drawn prior to ECMO cannulation ( $\mathrm{t}=$ =hours). In groups $1-4$, following ECMO cannulation further routine samples were drawn from the arterial line at timed intervals of $2,12,24,36$, 48, 72, 96 and 120 hours. In group 5 (48 hour cooling), samples were drawn at the same time points except at 60 instead of 36 hours.

\subsection{PROBLEMS IN OBTAINING CONTINUOUS QTC MEASUREMENT}

The directive given at the outset of the study was to measure the QTc interval continuously during cooling and rewarming. Clearly, standard 12 lead ECG's would not have given the continuous QTc measurement required. Since there was no existing QTc monitoring available on the PICU at the Glenfield Hospital, I explored various methods of achieving this aim. Experimentation with various methods of continuous QTc measurement took place during a four month period prior to commencement of the pilot study.

I initially explored the feasibility of downloading the ECG trace from the Hewlett Packard bedside monitor used to record the patient vital signs whilst on PICU at the Glenfield Hospital. I envisaged storing the data in a digitised form on a computer which could be later analysed.

This proved difficult for a number of reasons. Firstly, only two of the existing monitors on the PICU at the Glenfield Hospital had the appropriate interface to allow data to be extracted. This would have limited the amount of data I could have obtained since it would not have been practically possible to reserve these bed spaces for study use. I enquired about the cost of adding data interfaces to other monitors on 
the PICU, but the cost was prohibitive in terms of the funding available for the study. I did attempt to obtain a signal between the monitor interface and a laptop computer but despite the assistance of the Hewlett Packard Technical Team, I was unable to establish communication between the monitor and the computer.

Having abandoned this method of recording the QTc interval I explored the possibility of using a twenty hour Holter monitor to continuously record the QTc interval. I first attempted to record the ECG using a three electrode, two channel Holter monitor. The ECG was recorded onto a standard cassette tape which was then analysed by an experienced ECG technician using a Pathfinder analyser (Reynolds Medical). This proved a very unreliable method of obtaining an ECG trace of sufficient quality to analyse. The main problem was of electromagnetic interference both from the array monitors and infusion pumps surrounding the patient and the overhead radiant heater. Also, movement of the patient during physiotherapy or routine patient cares caused further disturbance of the recording.

Another difficulty with this recorder was that despite ensuring that both new batteries and a new cassette tape were inserted at the start of each recording, the recorder had a tendency to slow down. The consequence of slowing of the tape was that the ECG was recorded at a slower rate which suggested that the patient was very bradycardic when in reality their heart rate was normal. This resulted in an unreliable QT/QTc analysis.

I had several discussions with the manufacturers (Reynold Medical, Hertford UK) on ways to reduce the electrical interference on the tape. Firstly I tried using a specially adapted monitor which had much longer ECG leads allowing the monitor to be positioned further away from the patient. This gave some improvement but there was still intermittent interference which made analysis of sections of the recording 
impossible. After further discussions with the Technical Department at Reynolds Medical, I tried wrapping the ECG leads with silver foil to act as an insulator. The combination of longer ECG leads wrapped in foil with the monitor placed on a plastic table at the end of the cot seemed to give a better quality recording but this was still not completely reliable. However, at the time of commencement of the study, this was the best solution for obtaining continuous recording of the QTc.

By chance, just at the start of recruitment for the study, the ECG department at the Glenfield Hospital was in the process of acquiring digital 24 hour recorders to replace the older cassette recorders. I used the digital recorder on patient 4 (group 1) as an experiment to see if there was an improvement in quality and reliability of the recording. I found that using the digital recorder, there was no electrical interference and it was possible to obtain and analyse a continuous QTc recording. Another advantage of the digital recorder was that one could visualise and optimise the ECG trace prior to commencing the recording. Due to the improved quality and reliability of the recording, I acquired a digital recorder for the remainder of the patients recruited to the study. As a separate safeguard, I continued to record standard 12 lead ECG's as means of validating the accuracy of the digital QTc measurements. In view of the difficulties in obtaining a reliable method of continuous QTe recording, I recruited two additional patients. These patients were recruited after completion of stage 1 of the Pilot Study and included in the control group. 


\subsection{QT MEASUREMENT USING TWENTY FOUR HOUR DIGITAL ECG HOLTER RECORDING}

The ECG recordings were obtained using a three electrode, two channel Lifecard CF recorder (Reynolds Medical, Hertford UK). The electrodes were placed in standard positions recommended by the manufacturer. Prior to commencing the recording, the ECG trace was visualised on both channels of the monitor to ensure a good quality recording. The ECG was digitised and stored on a 30 MByte Flashcard (24 hour capacity) (Reynolds Medical, Hertford UK). Every twenty four hours, the Flashcard was changed, ECG electrodes replaced, a new battery inserted into the Lifecard monitor and the ECG trace optimised before continuing the recording.

The recordings were commenced within four hours following cannulation for ECMO and measured continuously for the first five days of the ECMO run.

Two experienced ECG technicians blinded to the patient temperature groups performed the QTc/QT analysis using a Pathfinder 700 analyser, software package version 8 (Reynolds Medical, Hertford UK).

The analysis in Pathfinder 700 is a true beat to beat measurement of QT dynamics. The QT interval was measured from the start of the Q to the end of $\mathrm{T}$ and the interval from the $Q$ to the apex of the $T$ wave (QTa) are measured for each beat.

The QRS complex was detected for each beat and using this as a starting point, the Q wave was detected by analysis of the slope of the signal. The end of the T-wave was detected by first detecting the peak of the T-wave. There must be only one apex in the T-wave. The end of the T-wave was then detected by analysis of the slope of the signal beyond the apex of $\mathrm{T}$. 


\section{Data Quality Criteria for Automated QT Analysis}

The beats used for QT analysis were selected as follows:

1. only beats classified by the pathfinder as " normal"

2. beats with an RR interval of $>2.5 \mathrm{~s}$ were excluded

3 . beats with a heart rate $<30 \mathrm{bpm}$ or $>160 \mathrm{bpm}$ were excluded

4. QT measurement was not performed on beats classified as aberrant, artefact or rate excluded as above. 


\subsection{THE DIGITAL ECG MONITOR AND FLASHCARD}

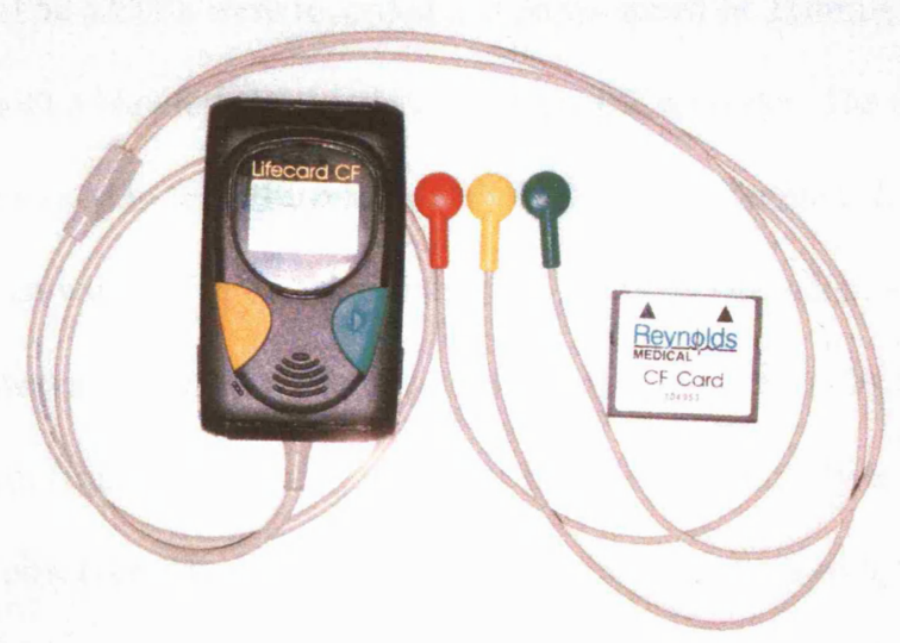

Standard Positions for ECG Electrodes

$\begin{array}{ll}\begin{array}{l}\text { Yellow } \\ \text { Red }\end{array} & \begin{array}{l}\mathrm{CH} 1 \text { - } \\ \mathrm{CH} 1+\end{array} \\ \begin{array}{l}\text { GREEN } \\ \text { Red }\end{array} & \begin{array}{l}\mathrm{CH} 2 \text { - } \\ \mathrm{CH} 2+\end{array} \\ \begin{array}{ll}\text { Yellow } \\ \text { GreEN }\end{array} & \mathrm{CH} 3 \text { - } \\ & \mathrm{CH}+\end{array}$

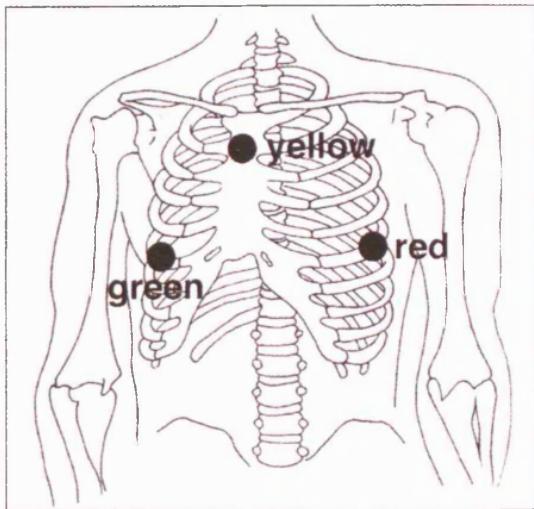




\subsection{TWELVE LEAD ELECTROCARDIOGRAM VALIDATION}

Twelve lead surface electrocardiograms (ECG's) were recorded daily for the first five days of ECMO. The ECG's were recorded at a paper speed of $25 \mathrm{~mm} / \mathrm{s}$ (amplitude $1 \mathrm{mV}=10 \mathrm{~mm}$ ) with a Hewlett- Packard Pagewriter 200i recorder. The QT intervals were usually measured in lead II from the start of the QRS complex to the end of the T wave. The mean value of three consecutive QT intervals was taken and the corrected QT interval (QTc) calculated by dividing the QT interval by the square root of the RR interval (Bazett's formula). All recordings were manually analysed using calipers by one observer. The interindividual accuracy was checked by duplicate measurement. Intra-observer accuracy of QT measurement was assessed in $50 \%$ of the recordings by two experienced observers blinded to the patient groups.

\subsection{STATISTICAL ANALYSIS}

Pre-ECMO clinical data was compared using the Kruskal-Wallis test.

The relation between temperature and QTc over the first 48 hours of the study period and during rewarming, was analysed using linear regression (Stata V.7 Statacorps, Tx USA). Differences in the QTc between individuals were accounted for using indicator variables. Potential covariates were assessed by univariate logistic or linear regression analysis.

The QTc values obtained using both manual and digital methods were compared and intraobserver reproducibility calculated using the Bland - Altman method. The mean difference and the limits of agreement for manual and automated values calculated as \pm 2 standard deviations are given in figure 1 . 


\subsection{RESULTS}

\section{Subjects}

The clinical characteristics of the twenty seven infants included in the study are summarised in table 1 . No significant difference was found between the five groups.

\section{Digital twenty Four Hour Monitoring}

Twenty four hour recordings were obtained in 25 of 27 patients (92\%).

There were no problems of electromagnetic interference from the ECMO equipment and nearby infusion pumps adversely affecting the quality of the digital recordings. Twenty of twenty five patients $(80 \%)$ completed $\geq 100$ hours of recording with 120 hours of recording obtained in 12 patients. Details of the automated QT and QTc interval measured in the first 24 or 48 hours of ECMO are shown in table 2. The scatter plots for digitally measured QTc against temperature for the 24 and 48 hour cooling groups are shown in figure 2.

During the first 24 hours of ECMO, the mean $\left(95^{\text {th }}\right.$ centile) values for the QTc interval at Group $137^{\circ} \mathrm{C}=431(506)$ milliseconds (ms); Group $236^{\circ} \mathrm{C}=459(521) \mathrm{ms}$; Group $335^{\circ} \mathrm{C}=445(516) \mathrm{ms}$; Group $434^{\circ} \mathrm{C}=465(531) \mathrm{ms}$; Group $534^{0} \mathrm{C}$ for 48 hours $=$ 466(521) ms.

Regression analysis of the QTc interval showed that there was an effect of temperature but the intra-individual variance of the QTc interval was large compared with the inter-group variance. Regression coefficient -3.12 milliseconds $(\mathrm{ms})^{-1}{ }^{0} \mathrm{C}^{-1}$ $(95 \% \mathrm{CI}-6.172--0.845) \mathrm{R}$-squared value $0.39(\mathrm{p}=0.04)$ 
There was no evidence that prolonging cooling from 24 to 48 hours affected the QTc interval.

\section{Lead Manual ECG Analysis}

Standard 12 lead ECG recordings were obtained daily for the first five days of ECMO in all patients. There were a total of 141 ECG traces of which 8 were excluded because the T waves were too flat to allow accurate QT measurement. Calculation of the intra-observer agreement of hand measured QT intervals showed a mean difference of 9 milliseconds ( $\mathrm{SD} \pm 0.03$ ) between values for compared $\mathrm{ECG}$ traces. Comparing the digital 24 hour and 12 lead ECG measurements, the mean difference between the two methods was -13.3 milliseconds and the limits of agreement +106.0 milliseconds and -132.7 milliseconds respectively (figure 1).

\section{The Effect of Temperature Change on the QTc Interval}

Overall, there was no significant relation between QTc and rectal temperatures during the rewarming period. However, some individuals showed a significant increase in the QTc during rewarming.

\section{Clinical Cardiovascular Complications}

The were no major problems of cardiovascular instability during the cooling and rewarming period. Five patients had a heart rate of $\leq 85$ beats/minute for at least one 
hour during cooling but remained normotensive. There were no episodes of cardiac arrhythmias in any of the infants during the study.

\section{Covariates}

The electrolyte values including sodium, potassium, calcium and magnesium were within the normal ranges for all patients during the cooling and rewarming period. Using univariate analysis, no relationship was found between QTc and electrolytes, heart rate and blood pressure. None of the patients were prescribed drugs such as cisapride. Inotropes were weaned and stopped within two hours of commencing ECMO although in two patients, inotropes were recommenced on day 2 of ECMO due to hypotension but stopped by day 3 ECMO.

\subsection{DISCUSSION}

The QT interval is a measure of ventricular depolarisation and repolarisation. Delayed repolarisation as indicated by prolongation of the QT interval can precipitate ventricular tachycardia including the potentially lethal torsade de pointes. In adults, hypothermia increases the QTc interval and is associated with supraventricular and ventricular arrhythmias. ${ }^{308-310}$ However, no study has systematically examined the effect of temperature on QT in infants.

Recent interest in mild hypothermia as a neuroprotective strategy for infants suffering perinatal hypoxia-ischaemia ${ }^{275}$ has made it more pressing to define the potential cardiovascular adverse effects of cooling, particularly following the report of one 
infant with a rectal temperature of $34.4^{0} \mathrm{C}$ had a prolonged QT interval of 570 milliseconds. $^{277}$

ECMO provides a good opportunity to study the effects of hypothermia, both because temperature is easily controlled, and because some patients undergoing ECMO will have suffered hypoxia-ischaemia. Indeed trials of cooling during ECMO to reduce neural injury are under consideration.

\section{Normal QTe values in Neonates}

Several studies have been conducted to determine normal limits for the paediatric ECG at $37^{\circ} \mathrm{C}^{311-313}$ with 440 milliseconds commonly used as the criterion for the upper limit of normal for the QTc interval, although on the basis of a small number of ECG recordings, Rijnbeek et $\mathrm{al}^{312}$ suggested an upper limit of normal for the QTc interval was 450 milliseconds. Comparing my values for digital QTc at different temperatures I found (as illustrated in table 2) that the median QTc interval for all temperature groups at $37^{\circ} \mathrm{C}$ were within the previously reported normal limits for QTc. However, during cooling and rewarming the QTc interval values in groups 2, 4 and 5 were wider than the reported upper limit of normal.

\section{Agreement Between Digital Monitor and 12 Lead ECG's}

Previous authors have used ambulatory electrocardiography (AECG) for measurement of the QT interval and the algorithms have been well validated. ${ }^{314-315}$ Also, with the improvement in monitor technology, the American College of Cardiology/ American Heart Association Task Force recently published guidelines for the use of AECG. ${ }^{316}$ 
However since there is (to my knowledge) no published data on the QT and QTc values in neonates measured by digital Holter monitoring, manual measurement of the QTc interval from standard 12 lead ECG's were used for validation of the digital values.

In my study, I observed that the digital QT and QTc values were slightly longer than the 12 lead ECG values. These differing values were possibly due to the fact that the 12 lead QT interval was a point value compared to the automated QT which was the average value over the previous hour. Conceivably, the hand measured method may have been more prone to observer error although the intraobserver mean difference for manual measurement was small. In addition, subtle differences in the alogorithms used to measure the QT interval may account for some of the difference in values. The Bland-Altman plots comparing the manually and automated measured QTc interval show reasonable agreement between the two methods and digital QTc values are valid to give a trend of the change of QTc interval with temperature.

\section{Mechanism of Prolongation of the QT Interval}

Overall, QTc interval changed slightly with temperature but the intra-individual variance was large, which suggests that factors other than temperature were influential on the QTc interval. This raises the question of the mechanism of QT widening during hypothermia and the potential contributing factors including bradycardia, conduction delay, and serum electrolyte levels.

Hypothermia causes atrioventricular conduction delay. Delayed atrioventricular conduction will result in bradycardia which in turn is known to increase the length of the QT interval. In my study, the QTc interval increased as the heart rate decreased. 
Evidence from animal experiments have shown that moderate hypothermia causes marked diastolic calcium loading resulting in impaired relaxation and decreased contractility. ${ }^{317}$ In guinea-pig ventricular myocytes prolongation of the QT interval during deep hypothermia was due to lengthening of the ventricular action potential. ${ }^{318}$ Such changes in the ventricular action potential may cause widening of the QTc interval.

I did not observe a consistent prolongation of the QT interval in patients cooled to the same temperature which may suggest differing conductive disturbance. Osborn $\mathrm{J}$ waves were not present on any of the electrocardiograms during cooling or rewarming. The presence of $\mathrm{J}$ waves are thought to be related to the severity of the temperature insult and the amplitude of the $\mathrm{J}$ wave notch a marker for phase 2 reentry in individuals inclined to develop various forms of early repolarisation syndrome. ${ }^{319}$ It is tempting to speculate that the absence of $\mathrm{J}$ waves in my study suggests no clinically relevant conduction disturbance occurred during mild hypothermia and hence a low risk of cardiac arrhythmias.

Electrolyte disturbance during cooling or rewarming may alter the cardiac muscle cell membrane potential and thus influence the QTc interval and possibly increase the risk of ventricular arrhythmias. I could find no relationship between the QTc interval and electrolytes. During ECMO the serum potassium, calcium and magnesium levels are kept within the normal ranges by intravenous supplementation.

\subsection{CONCLUSIONS}

QTc showed significant variability in individuals, and only a small proportion of this can be explained by the rectal temperature. During the period of rewarming, a few 
individuals showed a strong relationship between QTc and temperature change, but overall there was no significant relationship.

I have shown that the digital monitor can be used to measure QTc interval in neonates however, further validation studies are required to ascertain the normal limits for QTc values.

Despite widening of the QTc interval during mild hypothermia, the absence of major cardiovascular complications including arrhythmias will be reassuring to other researchers involved in cooling studies of neonates following hypoxic ischaemic injury. 


\subsection{FIGURES AND TABLES}

Figure 1 Bland Altman Plot Comparing the QTc Interval Measured by both Standard 12 lead ECG and Digital Monitoring

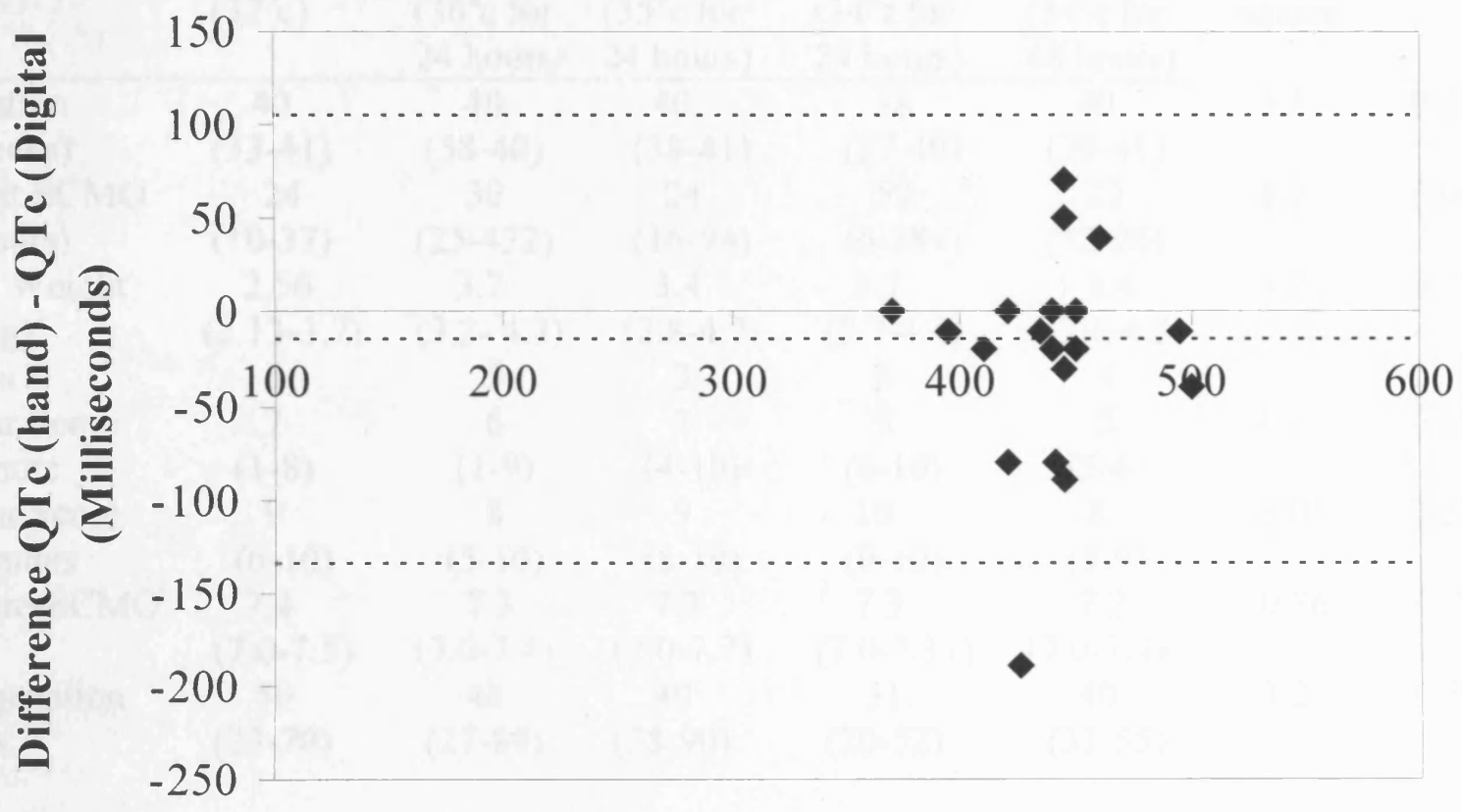

Mean QTe (Milliseconds) 
Table 1 Summary of Demographic Data for Study Groups

$(\mathrm{N}=7$ group $1 \mathrm{~N}=5$ per groups 2-5) Median values (Range)

\begin{tabular}{|c|c|c|c|c|c|c|c|}
\hline & $\begin{array}{l}\text { Group } 1 \\
\left(37^{\circ} \mathrm{c}\right)\end{array}$ & $\begin{array}{l}\text { Group } 2 \\
\left(36^{0} \mathrm{c} \text { for }\right. \\
24 \text { hours) }\end{array}$ & $\begin{array}{l}\text { Group } 3 \\
\left(35^{\circ} \mathrm{c} \text { for }\right. \\
24 \text { hours) }\end{array}$ & $\begin{array}{l}\text { Group } 4 \\
\left(34^{0} \mathrm{c} \text { for }\right. \\
24 \text { hours) }\end{array}$ & $\begin{array}{l}\text { Group } 5 \\
\text { ( } 34^{0} \mathrm{c} \text { for } \\
48 \text { hours) }\end{array}$ & $\begin{array}{l}\text { Chi- } \\
\text { square }\end{array}$ & P-value \\
\hline $\begin{array}{r}\text { Gestation } \\
\text { (weeks) }\end{array}$ & $\begin{array}{c}40 \\
(33-41)\end{array}$ & $\begin{array}{c}40 \\
(38-40)\end{array}$ & $\begin{array}{c}40 \\
(38-41)\end{array}$ & $\begin{array}{c}38 \\
(37-40)\end{array}$ & $\begin{array}{c}40 \\
(39-41)\end{array}$ & 5.5 & 0.238 \\
\hline $\begin{array}{l}\text { Age at ECMO } \\
\text { (hours) }\end{array}$ & $\begin{array}{c}24 \\
(10-37)\end{array}$ & $\begin{array}{c}30 \\
(25-432)\end{array}$ & $\begin{array}{c}24 \\
(16-94)\end{array}$ & $\begin{array}{c}50 \\
(6-384)\end{array}$ & $\begin{array}{c}22 \\
(12-26)\end{array}$ & 8.2 & 0.083 \\
\hline $\begin{array}{l}\text { Birth Weight } \\
(\mathrm{Kg})\end{array}$ & $\begin{array}{c}2.56 \\
(2.13-3.7)\end{array}$ & $\begin{array}{c}3.7 \\
(3.2-4.3)\end{array}$ & $\begin{array}{c}3.4 \\
(2.8-4.7)\end{array}$ & $\begin{array}{c}3.7 \\
(2.7-4.4)\end{array}$ & $\begin{array}{c}3.4 \\
(3.1-4.3)\end{array}$ & 4.2 & 0.378 \\
\hline Males & 5 & 5 & 3 & 3 & 4 & & \\
\hline $\begin{array}{l}\text { Apgar Score } \\
1 \text { minute }\end{array}$ & $\begin{array}{c}7 \\
(1-8)\end{array}$ & $\begin{array}{c}6 \\
(1-9)\end{array}$ & $\begin{array}{c}7 \\
(4-10)\end{array}$ & $\begin{array}{c}9 \\
(6-10)\end{array}$ & $\begin{array}{c}5 \\
(5-6)\end{array}$ & 6.2 & 0.183 \\
\hline $\begin{array}{l}\text { Apgar Score } \\
5 \text { minutes }\end{array}$ & $\begin{array}{c}9 \\
(6-10)\end{array}$ & $\begin{array}{c}8 \\
(5-10)\end{array}$ & $\begin{array}{c}9 \\
(8-10)\end{array}$ & $\begin{array}{l}10 \\
(9-10)\end{array}$ & $\begin{array}{c}8 \\
(8-9)\end{array}$ & 5.0 & 0.290 \\
\hline pH Pre-ECMO & $\begin{array}{c}7.4 \\
(7.0-7.5)\end{array}$ & $\begin{array}{c}7.3 \\
(7.0-7.4)\end{array}$ & $\begin{array}{c}7.3 \\
(7.0-7.7)\end{array}$ & $\begin{array}{c}7.3 \\
(7.0-7.31)\end{array}$ & $\begin{array}{c}7.2 \\
(7.0-7.4)\end{array}$ & 0.76 & 0.763 \\
\hline $\begin{array}{l}\text { Oxygenation } \\
\text { Index }\end{array}$ & $\begin{array}{c}50 \\
(23-70)\end{array}$ & $\begin{array}{c}48 \\
(27-89)\end{array}$ & $\begin{array}{c}40 \\
(28-90)\end{array}$ & $\begin{array}{c}31 \\
(20-52)\end{array}$ & $\begin{array}{c}40 \\
(31-55)\end{array}$ & 3.2 & 0.516 \\
\hline \multicolumn{8}{|c|}{ Primary Diagnoses } \\
\hline MAS/PPHN & 4 & 3 & 4 & 2 & 4 & & \\
\hline Sepsis & 2 & & & 1 & 1 & & \\
\hline $\begin{array}{l}\text { RDS } \\
\text { Congenital }\end{array}$ & 1 & & 1 & & & & \\
\hline $\begin{array}{l}\text { Diaphragmatic } \\
\text { Hernia (CDH) }\end{array}$ & & 1 & & 1 & & & \\
\hline $\begin{array}{l}\text { TAPVD * } \\
\text { Inborn Error of }\end{array}$ & & 1 & & & & & \\
\hline Metabolism & & & & 1 & & & \\
\hline
\end{tabular}

* Total anomalous pulmonary venous drainage

$\sim$ Myophosphorylase deficiency. (Possible Infantile $\mathrm{M}^{\mathrm{C}}$ Ardles type disorder) 
Table 2 Automated QT and QTc Interval During Cooling and Rewarming

\begin{tabular}{|c|c|c|c|c|c|}
\hline & $\begin{array}{l}\text { Group } 1 \\
37^{0} \mathrm{C} \text { for five } \\
\text { days }\end{array}$ & $\begin{array}{l}\text { Group } 2 \\
36^{0} \mathrm{C} \text { for } 24 \\
\text { hours } \\
\end{array}$ & $\begin{array}{l}\text { Group } 3 \\
35^{0} \mathrm{C} \text { for } 24 \\
\text { hours }\end{array}$ & $\begin{array}{l}\text { Group } 4 \\
34^{0} \mathrm{C} \text { for } 24 \\
\text { hours }\end{array}$ & $\begin{array}{l}\text { Group } 5 \\
34^{0} \mathrm{C} \text { for } 48 \\
\text { hours }\end{array}$ \\
\hline $\begin{array}{l}\text { Median QT (range) } \\
\text { milliseconds (ms) } \\
\text { for first } 24 \text { hours } \\
\text { ECMO }\end{array}$ & $\begin{array}{c}289 \\
(248-349)\end{array}$ & $\begin{array}{c}326 \\
(263-395)\end{array}$ & $\begin{array}{c}335 \\
(260-425)\end{array}$ & $\begin{array}{c}328 \\
(208-444)\end{array}$ & $\begin{array}{c}349 \\
(273-408)\end{array}$ \\
\hline $\begin{array}{l}\text { Median QTc (range) } \\
\text { ms for first } 24 \text { hours } \\
\text { ECMO }\end{array}$ & $\begin{array}{c}430 \\
(360-530)\end{array}$ & $\begin{array}{c}454 \\
(400-536)\end{array}$ & $\begin{array}{c}439 \\
(355-534)\end{array}$ & $\begin{array}{c}478 \\
(315-539)\end{array}$ & $\begin{array}{c}465 \\
(385-534)\end{array}$ \\
\hline $\begin{array}{l}\text { Median QT (range) } \\
\text { ms for first } 48 \text { hours } \\
\text { ECMO }\end{array}$ & Not Cooled & Rewarming & Rewarming & Rewarming & $\begin{array}{c}344 \\
(263-411)\end{array}$ \\
\hline $\begin{array}{l}\text { Median QTc (range) } \\
\text { ms for first } 48 \text { hours } \\
\text { ECMO }\end{array}$ & Not Cooled & Rewarming & Rewarming & Rewarming & $\begin{array}{c}466 \\
(385-534)\end{array}$ \\
\hline $\begin{array}{l}\text { Median QTc (range) } \\
\text { ms during } \\
\text { rewarming* }\end{array}$ & $\begin{array}{c}428 \\
(367-519)\end{array}$ & $\begin{array}{c}458 \\
(397-527)\end{array}$ & $\begin{array}{c}425 \\
(351-525)\end{array}$ & $\begin{array}{c}456 \\
(379-529)\end{array}$ & $\begin{array}{c}467 \\
(380-539)\end{array}$ \\
\hline $\begin{array}{l}\text { Median QTc (range) } \\
\text { ms when rewarmed } \dagger \\
\text { to } 37^{0} \mathrm{C}\end{array}$ & $\begin{array}{c}448 \\
(351-530)\end{array}$ & $\begin{array}{c}438 \\
(391-516)\end{array}$ & $\begin{array}{c}419 \\
(343-486)\end{array}$ & $\begin{array}{c}441 \\
(383-545)\end{array}$ & $\begin{array}{c}442 \\
(377-518)\end{array}$ \\
\hline
\end{tabular}

All values shown measured in milliseconds

*Denotes hours 25 - 49 of ECMO (except group 5 hours 49-72 ECMO)

†Denotes hours 49-72 of ECMO (except group 5 hours 73 -97 ECMO) 
Figure 2 Scatter Plots for Digital QTc for 24 hour Cooling Groups

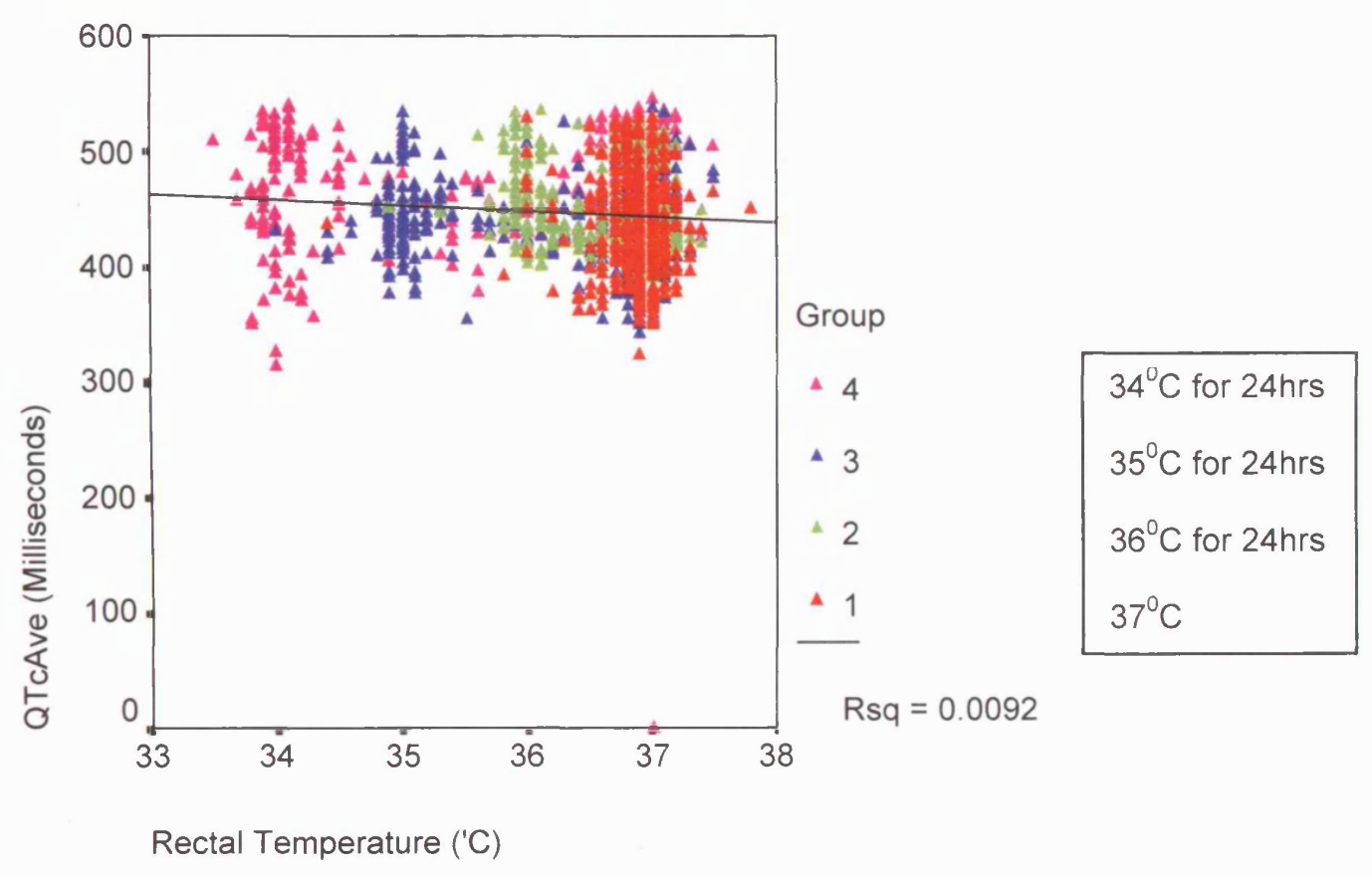

Figure 2 Scatter plot showing the digitally measured QTc in milliseconds for each individual patient in the 24 hour temperature groups. The graph displays the values of QTc for the control group and also during the cooling period (24 hours) and when rewarmed to $37^{\circ} \mathrm{C}$. 
Figure 3 Digital QTc for Group 5 (48 hr Cooling)

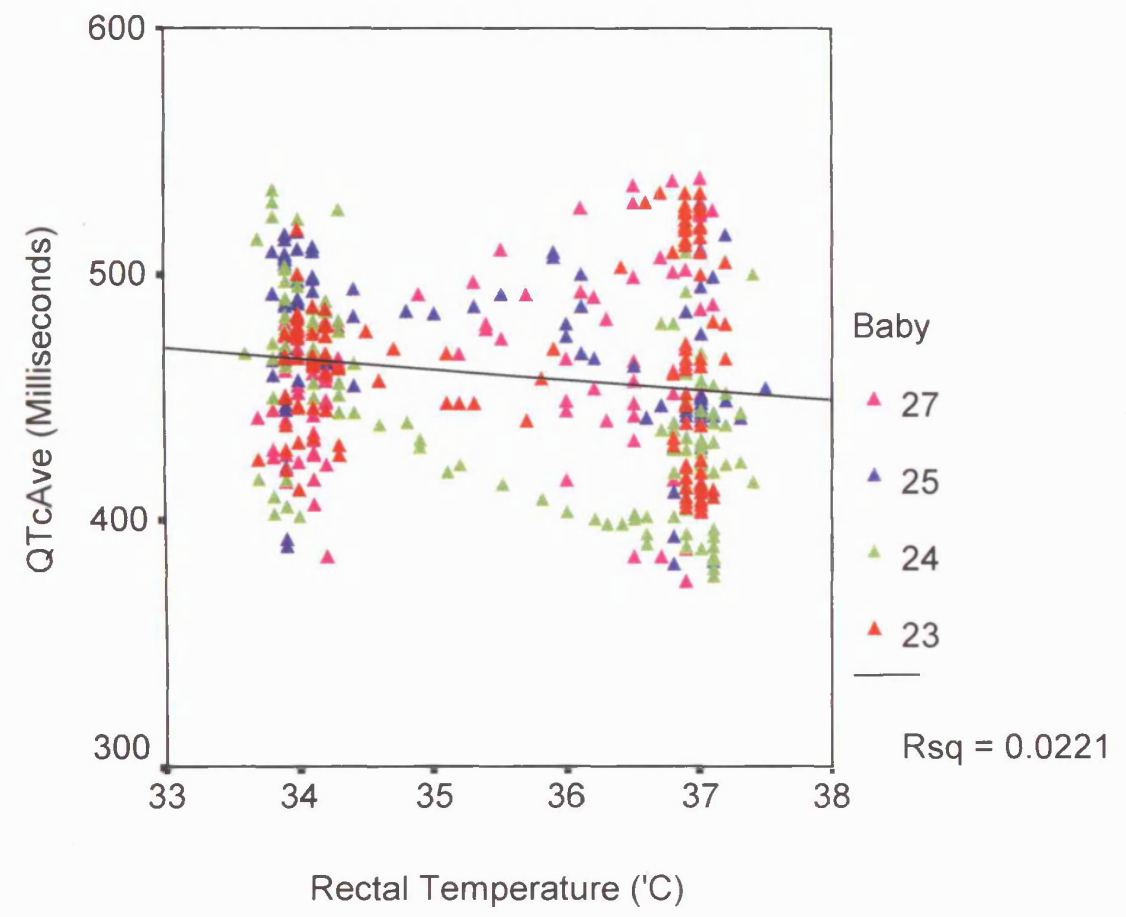

Figure 3 Scatter plot showing the digitally measured QTc in milliseconds for each individual patient in the 48 hour temperature groups. The graph displays the values of QTe for the patients in group 5 during cooling to $34^{\circ} \mathrm{C}$ for 48 hours and rewarming to $37^{\circ} \mathrm{C}$. 


\section{CHAPTER 6}

\section{THE EFFECT OF MILD HYPOTHERMIA ON COAGULATION}

\section{INTRODUCTION}

Coagulation dysfunction is a well described complication of hypothermia and a potential problem when cooling neonates on ECMO. The exact mechanism of hypothermic coagulopathy is not completely understood. Factors such as slowing of the enzymes involved in the coagulation cascade ${ }^{13}$ and decreased platelet function ${ }^{320}$ and numbers ${ }^{14}$ have been shown to be involved at least in part in the aetiology of hypothermic coagulopathy both in vivo and in vitro. In addition, the effect of temperature on clotting factor activation has been investigated as a factor contributing to the coagulopathy. A study by Reed et al, ${ }^{259}$ showed that hypothermia produced a kinetic slowing of clotting factor activity despite a normal level of the clotting factors. In a further study by Johnston et al, ${ }^{12}$ hypothermia at a temperature below $33^{\circ} \mathrm{C}$ was shown to produce a functional equivalence of a clotting factor deficiency, despite the presence of normal clotting factor levels.

In the context of applying hypothermia during ECMO, there are a number of factors which may contribute to an increased risk of coagulation problems both on the patient and the extracorporeal circuit. Patients receiving ECMO are routinely heparinised which might compound the possible increased risk of haemorrhage during cooling. Also the presence of metabolic acidosis, alterations in circulating calcium and $\mathrm{pH}$ values may contribute to dysfunction of haemostatic mechanisms. In addition, during cardiopulmonary bypass, haemodilution, consumption, and activation of the 
coagulation systems following exposure of blood to the circuit tubing increase the risk of bleeding. ${ }^{321-323}$

Also, the increased blood viscosity caused by cooling might interfere with the functioning of the membrane oxygenator or cause an embolus in the patient. Clearly, an important aim of the pilot study was to establish whether mild hypothermia caused major coagulation dysfunction or bleeding problems. One problem in achieving this aim was the concomitant factors such as metabolic acidosis, haemodilution and heparin made the assessment of the isolated contribution of hypothermia difficult. Furthermore, standardised laboratory coagulation testing is universally performed at the normothermic temperature of $37^{\circ} \mathrm{C}$ and therefore does not reflect the in vivo environment in which the hypothermic patients coagulation system must function.

\subsection{CHALLENGES OF MEASURING COAGULATION DURING HYPOTHERMIA}

\section{Measurement of Coagulation at "Real Temperature"}

As mentioned above, one of the challenges of obtaining meaningful coagulation data in cooled patients is that standardised laboratory coagulation studies are performed at $37^{\circ} \mathrm{C}$. The concern in designing the study was that coagulation measured at $37^{\circ} \mathrm{C}$ would not acurrately reflect the coagulation measured at the temperature of the blood in the patient i.e."real temperature". There is experimental evidence that when coagulation parameters are measured at "real temperature" during cooling there is a significant difference compared to the normothermic values. ${ }^{324,325}$ Data from 
Shimokawa et al ${ }^{325}$ showed that coagulation measured conventionally at $37^{0} \mathrm{C}$ overestimated the haemostatic function in neurosurgical patients during "mild hypothermia". Further work by Shimokawa et $\mathrm{al}^{324}$ in anaesthetised rabbits measured standard coagulation both at $37^{\circ} \mathrm{C}, 34^{\circ} \mathrm{C}$ and $30^{\circ} \mathrm{C}$. In addition, haemostatic function was tested at $37^{\circ} \mathrm{C}$ and $30^{\circ} \mathrm{C}$ using a sonoclot analyser and thromboelastography (TEG). The activated partial thromboplastin time was significantly higher during hypothermia to $30^{\circ} \mathrm{C}$ compared to $37^{\circ} \mathrm{C}$. The platelet count was substantially decreased in the hypothermic group. Similarly, the sonoclot and TEG experiments showed prolonged coagulation and clot forming times at $30^{\circ} \mathrm{C}$.

Similarly, Douning et $\mathrm{al}^{326}$ used a thromboelastograph modified with a thermostat to perform the test at the patient's body temperature. In this study of 45 patients undergoing liver transplantation, there was significant prolongation of the coagulation time and decreased clot formation rate when the patient's body temperature was less than $37^{\circ} \mathrm{C} .^{326}$

\section{Consideration of Temperature in Coagulation Tests for the Pilot Study}

The laboratory at the Glenfield Hospital were unable to measure the routine coagulation studies including INR, prothrombin time (PT), APTT and fibrinogen at temperatures other than the standard temperature $37^{\circ} \mathrm{C}$. In view of this, I investigated other methods of obtaining additional coagulation data which was either temperature independent or measured at the real temperature of the patient. 


\subsection{MOLECULAR MARKERS OF COAGULATION}

\section{Plasmin-o2-plasmin complex (PAP)}

Fibrinolysis is the process by which fibrin formed in the circulation is enzymatically degraded into soluble products. The removal of polymerized fibrin from the vascular system is important for maintaining the haemostatic balance. The key enzyme of the fibrinolytic system is plasmin which is formed from plasminogen. The main action of plasmin is to digest fibrin in thrombus to fibrin degradation products. Plasmin is effectively bound in the plasma by its inhibitor $\alpha 2$-antiplasmin, forming the plasmin/ $\alpha 2$-antiplasmin complex (PAP). The plasma PAP concentration is thus a measure of the current activity of the fibrinolytic system. An increase in PAP concentrations have been reported in patients with disseminated intravascular coagulation (DIC).

\section{Thrombin/antithrombin III complex}

The conversion of prothrombin into active thrombin is a key event in the coagulation cascade. In addition to converting fibrinogen to fibrin, thrombin acts on the platelets (to cause irreversible aggregation); it also alters the factor VIII molecule (accelerating the earlier stages of the cascade) and activates factor XIII (ensuring the stability of forming fibrin). Thrombin is inhibited by antithrombin III: this results in an inactive proteinase/inhibitor complex. The clinical significance of the determination of thrombin/antithrombin III (TAT) complex is in the diagnosis of thrombotic events in patients predisposed to thrombosis and disseminated intravascular coagulation (DIC) 


\section{Antithrombin III}

Antithrombin III is the plasmatic inhibitor of thrombin and activated Factor $\mathrm{X}$ which forms an irreversible inactive complex with these enzymes. Acquired antithrombin III deficiency occurs due to consumption following major surgery or due to DIC.

My aim was to use the molecular markers of coagulation to detect signs of activation of the coagulation system. In particular, these markers would allow for comparison between the groups which was independent of the temperature at the time that the blood sample was taken.

\subsection{THE ACTIVATED CLOTTING TIME}

Heparin is administered continuously in the ECMO circuit to avoid clotting anywhere inside the tubing surface. Heparin will prolong the clotting time of whole blood by blocking the clot formation at various sites in the normal coagulation system, notably that of prothrombin to thrombin and fibrinogen to fibrin.

By inhibiting the final clot, blood can freely circulate within the ECMO circuit. Excessive heparin administration can inhibit the clotting cascade such that the patient may haemorrhage. The site of haemorrhage will vary with the individual patient but include pulmonary, gastric, cerebral, ECMO incision and any other surgical sites. It is important to maintain the clotting time at a level that will avoid thrombosis formation in any portion of the ECMO circuit, while not creating bleeding complications for the patient. 
The activated clotting time (ACT) test, first described by Hattersley in $1966,{ }^{327}$ is a rapid bedside test for clotting. For this study, the ACT was measured during ECMO using the Hemochron machine (International Technidyne, Inc).

\section{ACT Measurement Using Hemochron}

Paediatric tubes were used which contained ground glass as the activator, a small magnet and internal plastic ridge. $0.5 \mathrm{ml}$ of whole blood was aspirated from the ECMO circuit. The hinged cap was flipped open, the activator powder gently tapped to the bottom of the tube, the blood sample injected into the bottom of the tube and the cap closed. The timer was started on the machine and the tube quickly agitated whilst holding the tube upright. After 10 seconds, the tube was inserted into the receptacle in the hemochron and rotated clockwise until the green "dectector" light was illuminated. The tube was rotated another complete turn to ensure that the magnet moved freely. The timer stopped when clot stabilisation advanced sufficiently to trap the cylindrical magnet against the plastic ridge. The blood was warmed to $37^{\circ} \mathrm{C}$ during the analysis.

The normal range for an ACT in a non-heparinised patient is $90-150$ seconds. The desired value is based on the clinical state of the patient and the bleeding risk, but is usually in the range of 180-240 seconds.

\section{Measuring ACT During Mild Hypothermia}

In an attempt to overcome the problems of measuring coagulation at the "real" temperature of the patient, I investigated the possibility of modifying the hemochron 
machine to measure the ACT over a range of temperatures. I liased with the Engineering department at the University of Leicester. Unfortunately, it was not possible to modify the temperature control within the Hemochron machine. This was mainly due to technical difficulties in obtaining a thermistor which could maintain a steady cooled state. 


\subsection{THE HEMOCHRON ACT MONITOR}

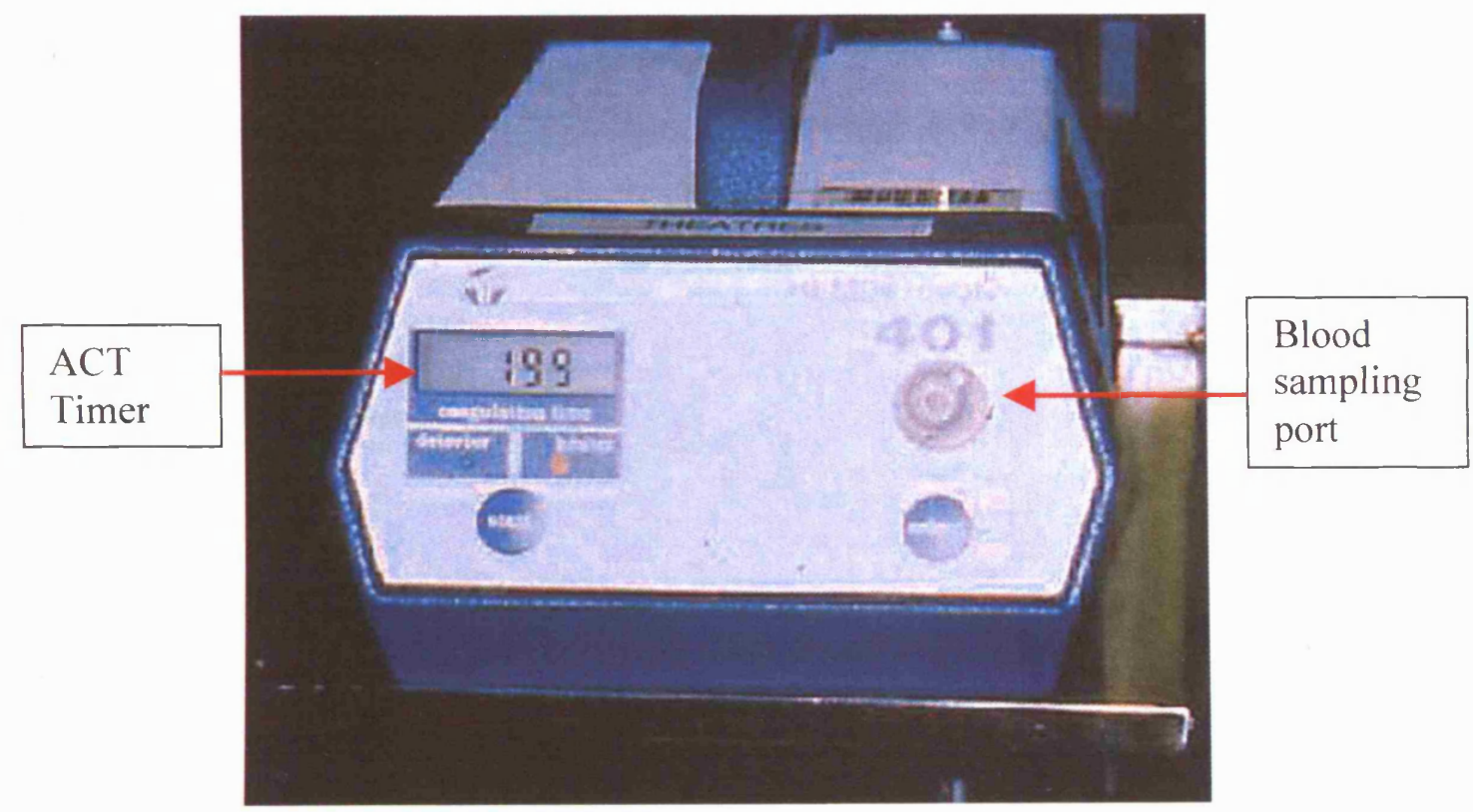




\subsection{METHODS}

\section{Study Groups}

The study included the twenty five patients described in chapters 3 and 4 . The entry criteria and ECMO management are described in chapter 3 . The study groups were identical to those described in chapter 3.

\section{Hypothermic Treatment}

The infants were cooled and rewarmed by adjusting the temperature of the water bath in the extracorporeal circuit as described in chapter 3.

\section{Monitoring and Blood Sample Collection}

Rectal and abdominal skin temperature were monitored continuously. Arterial blood pressure, ECG, respiratory rate and pulse oximetry were shown continuously on the monitor screen (Hewlett Packard HP Component Monitoring System Model 66S) according to our routine practice. Routine coagulation samples including INR, APTT, fibrinogen, prothrombin time and D-dimer were drawn prior to ECMO cannulation $(\mathrm{t}=0$ hours). In groups $1-4$, following ECMO cannulation further routine coagulation samples were drawn from the arterial line at timed intervals of $2,12,24,36,48,72$, 96 and 120 hours. In group 5 (48 hour cooling), samples were drawn at the same time points except at 60 instead of 36 hours. In all samples, to prevent heparin contamination, $2 \mathrm{ml}$ of blood was first drawn from the arterial line before obtaining 
the study sample. The unused "dead space" blood was then returned to the patient via the arterial line.

In addition at the same time intervals, blood was drawn for more specialised tests of coagulation including molecular markers of coagulation, thrombin-antithrombin III (TAT), plasmin- $\alpha 2$ plasminogen (PAP) and antithrombin III. These were centrifuged at $3000 \mathrm{RPM}$ at $4^{0} \mathrm{C}$ for 15 minutes and stored at $-70^{\circ} \mathrm{C}$ until further analysis.

The activated clotting time was measured at least hourly or more often as clinically indicated. Finally the heparin and platelet requirements were recorded 2, 12 and 24 hourly.

\section{Measurement of Molecular Markers of Coagulation}

Thrombin/antithrombin III (TAT) and plasmin- $\alpha 2$ plasminogen (PAP) (Enzygnost ${ }^{\circledR}$ Dade Behring, Marburg, Germany) were analysed by ELISA as per the manufacturer's instructions. The lowest sensitivities of the assays were $2 \mu \mathrm{g} / \mathrm{L}$ and $50 \mu \mathrm{g} / \mathrm{L}$ respectively.

Antithrombin III (AT III) (APTEC® Sigma diagnostics, Dorset UK) was analysed by turbidimetric immunoassay (lowest senility $20 \mathrm{mg} / \mathrm{dl}$ ).

All the assays were performed by Ms Rachel Hodge, (Department of Child Health, Leicester University) who has considerable expertise and experience in ELISA methodology. 


\section{6 $\underline{\text { RESULTS }}$}

There were no major problems of bleeding during the study. One patient in group 2 had bleeding around the cannula site for several hours post ECMO cannulation. This was related to heparin given inadvertently early during cannulation and resolved after correction with protamine. Patient 22 in group 5 had a marked coagulopathy with deranged activated clotting times (ACT) between 400 to 700 seconds from the time of ECMO cannulation. The coagulopathy gradually resolved and ACT decreased to 187 seconds by 12 hours of ECMO following colloid infusions, a bolus of vitamin $\mathrm{K}$ and suspension of the heparin infusion. Cooling was continued for 48 hours and the patient rewarmed without further problems of coagulopathy, deranged ACTs or bleeding.

In all other patients, activated clotting time was initially prolonged but corrected to between 160 and 200 seconds by adjusting the heparin dosage according to the unit protocol.

The median (range) total requirements for platelet transfusion for the initial five days were: $109.3(71.6-121.9) \mathrm{ml}$ in group $1 ; 78.8(76.3-85.1) \mathrm{ml}$ in group $2 ; 60.7(46-73.7)$ $\mathrm{ml}$ in group $3 ; 88.6(71-132) \mathrm{ml}$ in group $4 ; 69.3(55.9-80.2) \mathrm{ml}$ in group 5 . The mean (SD) total requirements for heparin for the initial five days were: $358(234)$ units $/ \mathrm{kg}$ in group $1 ; 388(230)$ units/kg in group $2 ; 295(174)$ units/kg in group $3 ; 288$ (199) units $/ \mathrm{kg}$ in group $4 ; 330(227)$ units/kg in group 5. A scatter plot showing heparin requirements for the study groups are shown in figure 1 . However, all patients in group $5\left(34^{0} \mathrm{C}\right.$ for 48 hours $)$ showed a consistent rise then fall in heparin requirement during the cooling and rewarming period $(0-60$ hours) (see figure 2$)$. The significance of this observation is not clear. 
Thrombin-antithrombim III and plasmin- $\alpha 2$ plasminogen showed no particular trend with changing temperature (see Figure 3).

Also no trend with temperature was noted for each group with regard to INR, Ddimer, APTT and prothrombin time (see Figure 4).

\subsection{DISCUSSION}

None of the patients had major bleeding problems during cooling or rewarming but there were several observations which require further comment.

Laboratory investigation of coagulation and the activated clotting time (ACT) used at the cotside to monitor heparin levels were measured at $37^{\circ} \mathrm{C}$. Laboratory investigation of coagulation and the activated clotting time (ACT) used at the cotside to monitor heparin levels were measured at $37^{\circ} \mathrm{C}$. As mentioned in the introduction to this chapter, a recent report of the influence of induced hypothermia on haemostatic function in rabbits found that conventional coagulation measurement conducted at

$37^{\circ} \mathrm{C}$ failed to detect hypothermic-induced degradations in haemostatic perfomance. ${ }^{324}$ In addition, the ACT was prolonged when measured at real temperature $\left(30^{\circ} \mathrm{C}\right) .{ }^{324}$ Measuring the activated clotting time at $34^{\circ} \mathrm{C}$ may give a more accurate assessment of heparin requirement during the cooling period. A potentially interesting finding factor in this study was the potential additive effect of heparin on coagulation during cooling. No previous studies have examined the additive effect of heparin on coagulation during cooling. However it is tempting to speculate that clotting might be further prolonged in heparinised blood during cooling. The consistent rise and fall of heparin seen in group 5 may possibly suggest that less heparin may be required during 
cooling. Further investigation will be required to assist with accurate heparin management during cooling on ECMO.

Previous studies have demonstrated lowering of platelet levels and impaired platelet aggregation during and after cardiopulmonary bypass. ${ }^{328}$ There were no differences in platelet requirements between groups during my study. Similarly, Boldt et $\mathrm{al}^{328}$ reported an increase in thrombin/antithrombin III complex levels in hypothermic patients suggesting activation of the coagulation system. In my study there was a fall in the thrombin/antithrombin III level during the first 48 hours of ECMO.

These differences in findings may be explained by the use of mild hypothermia in my investigation compared to deep hypothermia $\left(22-32^{\circ} \mathrm{C}\right)$ in the reported studies. Further investigations are required of the effect of mild hypothermia on platelet function and activation of coagulation in larger studies to clarify this finding. The markedly deranged ACTs in patient 2 group 5, were probably due to the use of a new hollow fibre oxygenator rather than an effect of temperature. This was almost the first experience of using this type of oxygenator at the Leicester ECMO Centre and the routine heparin protocol was used. This resulted in overheparinisation and the ACT management when using this oxygenator is under review. Consideration was given to rewarm the infant, but after discussion with the ECMO director, cooling was maintained for 48 hours without clinical sign of haemorrhage or further derangement of the ACTs.

\subsection{CONCLUSIONS}

In this pilot study, there were no major problems with bleeding in infants receiving ECMO and cooled to $34^{\circ} \mathrm{C}$. None of the coagulation parameters measured showed any change with temperature. However, more work is needed in the area of measuring 
the coagulation factors at "real temperature" which may alter the heparin management of infants who are cooled during ECMO. 


\subsection{FIGURES AND TABLES}

Figure 1 Heparin Requirements for Study Groups

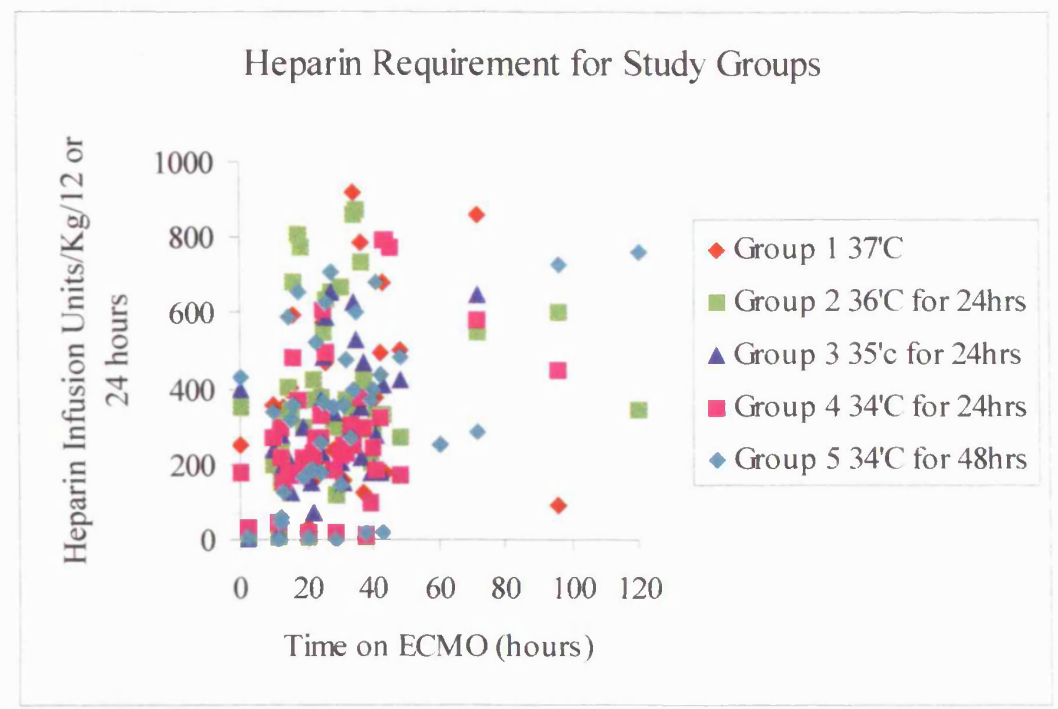

Figure 1 Scatter plot showing the heparin requirements for each individual patient in the five temperature groups. The graphs display the values heparin infused for the control group also during the cooling period (24 or 48 hours) and when rewarmed to $37^{\circ} \mathrm{C}$.

Figure 2 Heparin Requirement for Group 5

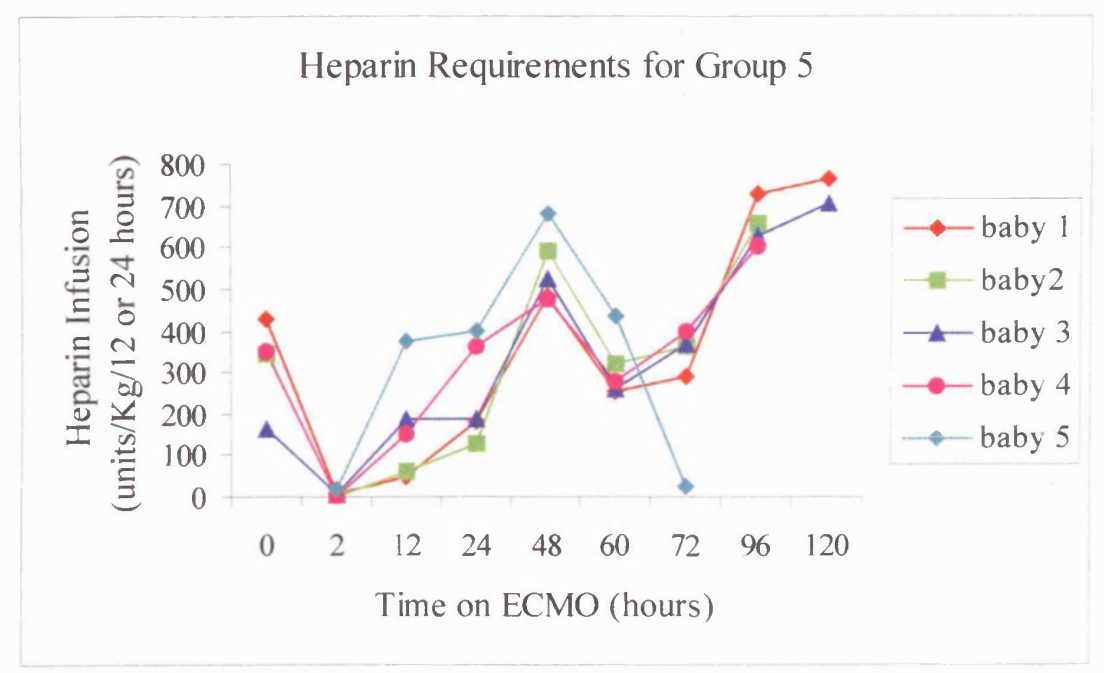

Figure 2 Graph showing the total amount of heparin infused for each patient in group 5 for the time points shown during the study period.

** Baby 5 was decannulated from ECMO by 72 hours 
Figure 3 Molecular Markers of Coagulation

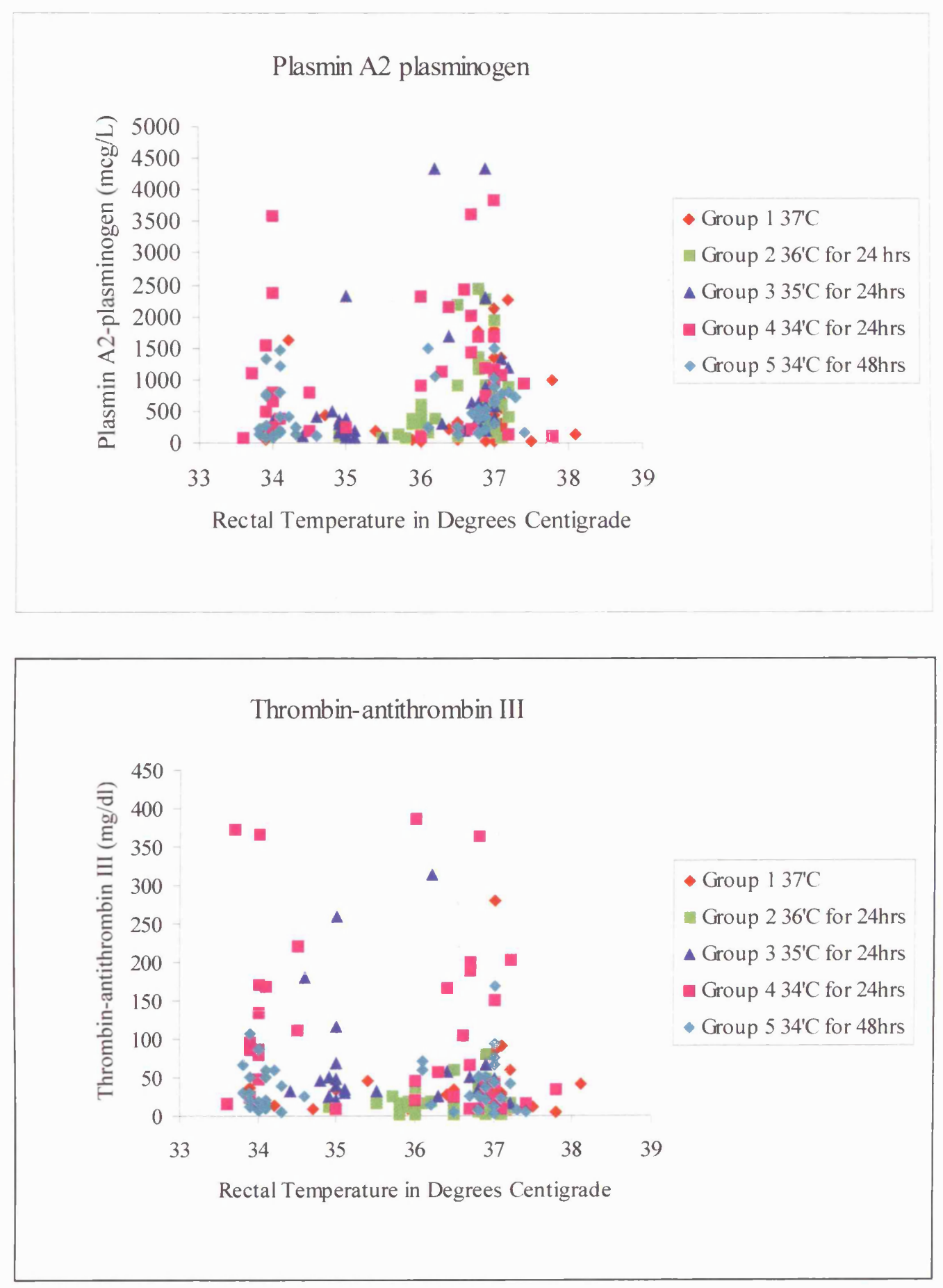

Figure 3. Scatter plots showing plasmin- $\alpha 2$ plasminogen (PAP) and thrombinantithrombin III (TAT) measurements for each individual patient in the five temperature groups. The graphs display the values of PAP and TAT for the control group also during the cooling period ( 24 or 48 hours) and when rewarmed to $37^{\circ} \mathrm{C}$. 
Figure 4 Coagulation Parameters for Study Groups
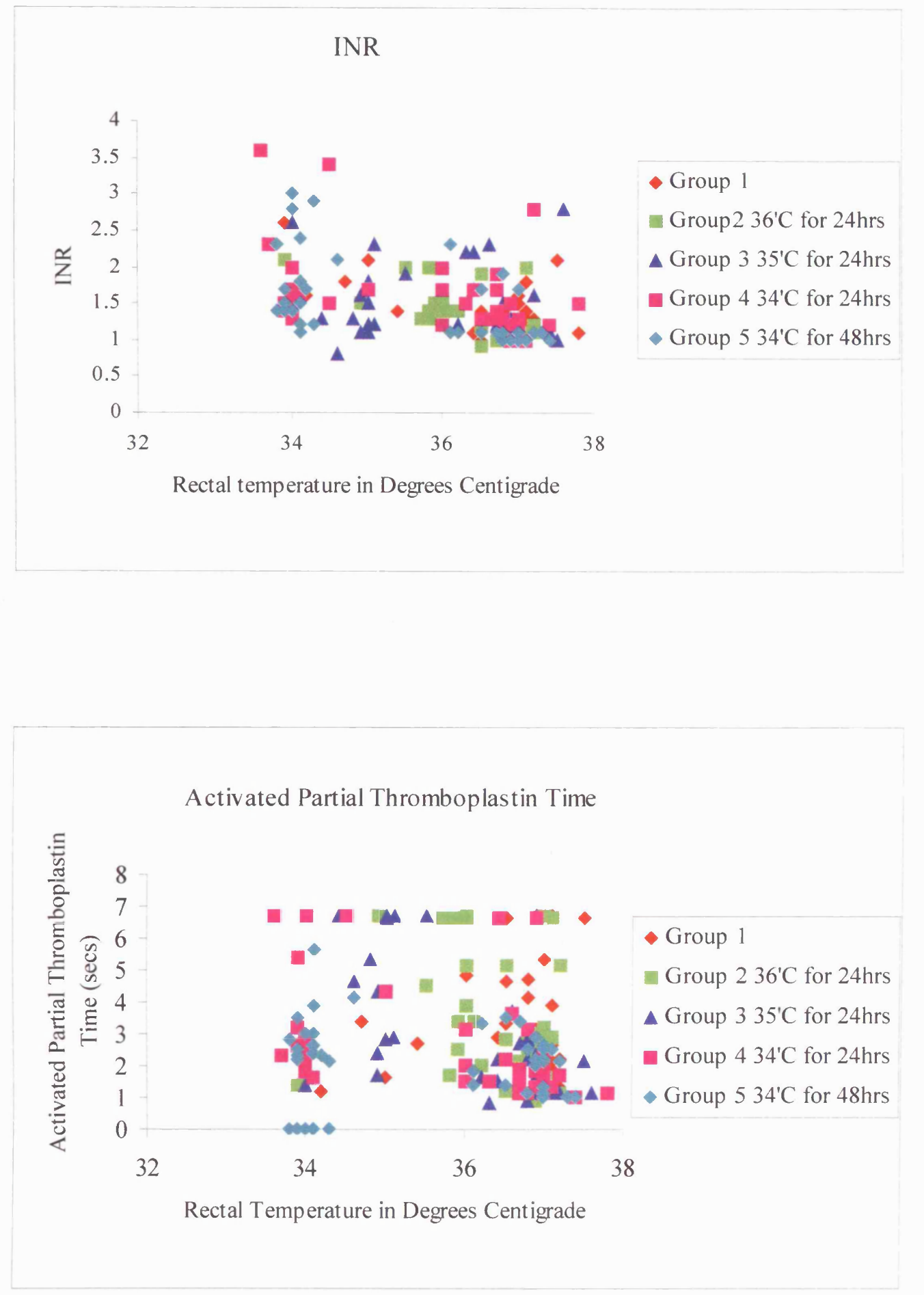
Figure 4 Coagulation Parameters for Study Groups

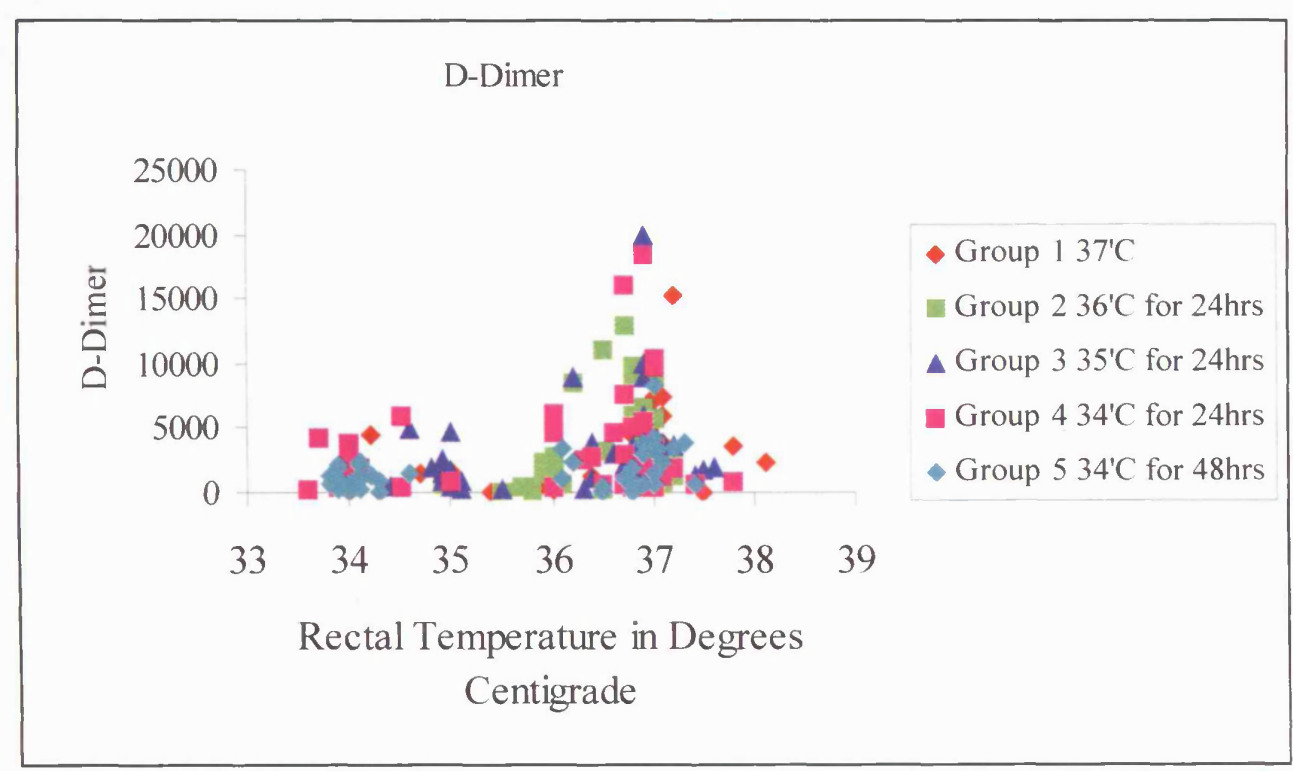

Figure 4 Scatter plots showing INR, activated partial thromboplastin time and DDimer measurements for each individual patient in the five temperature groups. The graphs display the values of INR, activated partial thromboplastin time and D-Dimer for the control group also during the cooling period ( 24 or 48 hours) and when rewarmed to $37^{\circ} \mathrm{C}$. 


\section{CHAPTER 7}

\section{CARDIOVASCULAR CHANGES DURING MILD HYPOTHERMIA AND REWARMING}

\section{INTRODUCTION}

The potential adverse effects of applying mild hypothermia in clinical practice include cardiovascular complications.

In humans, most of the documented effects on the cardiovascular system are during accidental hypothermia ${ }^{329}$ and deep hypothermia during cardiopulmonary bypass or neurosurgery. Mild hypothermia causes a sympathetic driven response with an initial tachycardia, peripheral vasoconstriction and a consequent increase in cardiac output and blood pressure. ${ }^{330}$ In vitro cooling of pig myocardium to $32^{\circ} \mathrm{C}$ causes a prolongation of contraction, and an increase in contractile force by almost $40 \%$. ${ }^{331}$ During moderate hypothermia, progressive bradycardia develops due to slowing of spontaneous depolarization of the pacemaker cells. There is a resultant reduction in cardiac output which may be balanced by an increased systemic vascular resistance consequent on autonomic reflex response and catecholamine release. The increased systemic vascular resistance may be perpetuated by increased viscosity and local vasomotor responses. ${ }^{332}$ Reuler et $\mathrm{al}^{333}$ described increased resistance in renal arteries in animals but vasodilatation in the splanchic vasculature. ${ }^{333}$

Repolarisation abnormalities occur as seen by the appearance of Osborn J waves on the ECG. ${ }^{334}$ Osborn J waves are however not pathognomonic of hypothermia and are also seen in conditions such as cardiac ischaemia and subarachnoid haemorrhage. Also during accidental hypothermia the ECG can show increasingly broad complex QRS complexes, indicating slowing of myocardial conduction. There may be 
associated ST elevation or depression and T wave inversion which can be related to increasing acidosis or ischaemia. ${ }^{334}$ At a cellular level, there is prolongation of the action potential duration due to delayed activation of the repolarising potassium current, slowed inactivation of the sodium current and delayed inactivation of the inward calcium current. ${ }^{335}$ There is prolongation of systole and delay in conduction may be reflected by an increase in the PR interval. ${ }^{336,337}$ Prolongation of the QT interval has also been described in moderate and deep hypothermia. ${ }^{338}$ Hypothermia slows the atrial pacemaker and intracardiac conduction. Consequently, hypothermia to less than approximately $35.5^{\circ} \mathrm{C}$ is associated with mild but sustained bradycardia. ${ }^{256,339-340}$ In deep hypothermia $\left(28^{\circ} \mathrm{C}\right)$, the heart rate falls to $30-40$ beats per minute..$^{341-342}$ Bradycardia may become extreme with rates of 10 beats per minute described at $20^{\circ} \mathrm{C}$. Systemic vascular resistance falls as catecholamine release is blunted and cardiac output decreases correspondingly. At temperatures less than $24^{\circ} \mathrm{C}$ there is a high risk of asystole. Gilbert et $\mathrm{al}^{343}$ described a case of an adult who fell into an icy gully. Her core temperature was $13.7^{\circ} \mathrm{C}$ and she was asystolic but successfully resuscitated over a period of 9 hours using an extracorporeal circuit.

Ischaemia, increased adrenergic activity and electrolyte disturbance predispose to cardiac irritability and in moderated hypothermia this frequently results in arrhythmias, commonly atrial flutter or fibrillation but also ventricular tachyarrhythmias. ${ }^{329}$ Ventricular fibrillation is more common below $27^{0} \mathrm{C}$ and may be due to the development of a temperature gradient between the cooler endocardium and subendocardial conducting tissue. The relatively warmer myocardium facilitates conduction through the myocardium at the expense of the normal conducting pathway which may explain why sudden changes in electrolytes and acid base affect the tissues differently and predispose to the development of ventricular fibrillation. ${ }^{329}$ 
Although there is considerable information about the cardiovascular effects of accidental hypothermia particularly in adults and deep hypothermia during cardiopulmonary bypass, there is limited data on the cardiovascular changes in humans during prolonged induced mild hypothermia. Experimentally, systemic cooling increases the release of catecholamines, peripheral vasoconstriction and systemic arterial pressure. Thoresen et $\mathrm{al}^{275}$ reported a series of nine infants with hypoxic-ischaemic encephalopathy cooled by either local head cooling $(N=6$ target temperature 34 to $35^{\circ} \mathrm{C}$ ) or systemic cooling $\left(\mathrm{N}=3\right.$ target temperature 33 to $\left.34^{\circ} \mathrm{C}\right)$ for a median of 72 hours. The mean arterial blood pressure increased by a median of $10 \mathrm{mmHg}$ during cooling and fell by a median of $8 \mathrm{mmHg}$ on rewarming. Heart rate decreased by a median of 34 beats/minute on cooling and increased by a median of 32 beats/minute on rewarming. No cardiac arrhythmias were reported. In a pilot study of whole body hypothermia in ten neonates, Azzopardi et al ${ }^{344}$ reported no cardiac arrhythmia or significant hypotension during cooling and rewarming.

No previous studies have investigated the cardiovascular changes during mild hypothermia in neonates receiving ECMO. Therefore as part of the pilot study, I systematically documented the cardiovascular changes during mild cooling and rewarming on ECMO. 


\subsection{METHODS}

\section{Study Group}

The study included the twenty five patients described in chapters 3 and 4 . The entry criteria and ECMO management are described in chapter 3 . The study groups were identical to those described in chapter 3 .

\section{Hypothermic Treatment}

The infants were cooled and rewarmed by adjusting the temperature of the water bath in the extracorporeal circuit as described in chapter 3.

\section{Cardiovascular and Temperature Monitoring}

Rectal and abdominal skin temperature were monitored continuously. Arterial blood pressure, ECG, respiratory rate and pulse oximetry were shown continuously on the monitor screen (Hewlett Packard HP Component Monitoring System Model 66S) according to our routine practice. The heart rate, arterial blood pressure and rectal temperature were recorded hourly by the ECMO Specialist.

The total colloid requirements for each study group were recorded. 


\subsection{STATISTICAL ANALYSIS}

Comparison between groups were made using the Kruskal-Wallis test. P value at 0.005 level was considered statistically significant.

\subsection{RESULTS}

The details of the heart rate and blood pressure values for all groups before and during cooling are shown in tables 1 and 2 . There was no significant difference between groups in terms of the heart rate or blood pressure prior to cooling. The mean arterial blood pressure increased following the commencement of ECMO but this increase was not statistically significant. Inotropes were weaned and stopped within two hours of commencing ECMO. In patient 16 (group 4) and patient 14 (group 3), inotropes were recommenced on day 2 of ECMO due to hypotension. The mean (SD) colloid requirements for the study groups were: 84 (75) $\mathrm{ml}$ in group $1 ; 84$ (78) $\mathrm{ml}$ in group 2; $70(58) \mathrm{ml}$ in group $3 ; 117(101) \mathrm{ml}$ in group $4 ; 69(51) \mathrm{ml}$ in group 5. There was no significant difference between the five groups (Kruskal-Wallis chi-squared 2.08, $\mathrm{df}=4$, $\mathrm{p}=0.72)$.

Five patients had a heart rate $\leq 85$ beats/minute for at least one hour during cooling but remained normotensive. There were no episodes of cardiac arrhythmia.

Two patients (numbers16 and 20 in group 4) were tachycardic on admission to the PICU and remained tachycardic throughout cooling and rewarming. Both patients remained in extremis during the initial 12 hours of ECMO with persistent metabolic acidosis and clinical concern of sepsis. In a third patient (No 25 in group 5) the heart rate was observed to decrease to below 95 beats/minute for 18 of the first 29 hours of 
cooling to $34^{\circ} \mathrm{C}$. From hour 30 to the end of cooling at 48 hours on ECMO, there was an increase in the heart rate from 115 to 151 beats/minute (see figure 1). The heart rate was 170 beats/minute at the time of decannulation from ECMO at 62 hours. The infant deteriorated 12 hours post ECMO decannulation with signs of sepsis including an increasing oxygen requirement and a raised C-reactive protein of 138mg/L.

\subsection{DISCUSSION}

Overall there were no major problems of cardiovascular instability during cooling or rewarming. In all groups we observed an increase in the mean arterial blood pressure (MABP) once the patients were stabilised on ECMO. All patients remained normotensive throughout cooling but two patients (cooled for 24 hours) required inotrope support on day 2 of ECMO for hypotension.

Previous cooling studies have reported an increase in the MABP on cooling with a decrease in MABP during rewarming. ${ }^{275,344}$ These were studies performed in infants not on ECMO. During ECMO blood pressure is aggressively managed and hence we cannot be certain that the changes in MABP we observed really represent the effects of cooling. However, we did not use significantly larger quantities of colloid in the babies cooled to $34^{0} \mathrm{C}$ for 24 or 48 hours during either cooling or rewarming. A sinus bradycardia was noted during cooling in the majority of patients in groups 3 to 5 . The heart rate increased on rewarming and there were no episodes of cardiac arrhythmias. These findings are similar to the heart rate changes reported in previous studies of cooling in neonates with hypoxic-ischaemic encephalopathy. ${ }^{275.344}$ In group $2\left(36^{\circ} \mathrm{C}\right.$ for 24 hours $)$ the median heart rate during cooling was lower than that reported by Gunn et al. ${ }^{277}$ Although, there was an increase in the heart rate with 
rewarming, the median heart rate in group 2 remained between $100-121 \mathrm{~b} / \mathrm{min}$ to 96 hours. This may be explained by the effect of sedation in that four of the infants in this group remained on ECMO for more than 100 hours and were maintained on both midazolam and morphine infusions throughout this time.

One interesting and potentially important observation, was of persistent tachycardia during cooling in two infants in group 4 and one in group 5. The two infants in group 4 subsequently died and the patient in group 5 showed clinical signs of sepsis with raised serial C-reactive protein measurements.

The numbers are too small to allow statistical analysis of heart rate and outcome. However, I would suggest that during future studies of mild hypothermia, patients with a persistent tachycardia should be carefully monitored for an underlying cause particularly sepsis.

\subsection{CONCLUSIONS}

There were no major cardiac complications during cooling. In particular there were no episodes of cardiac arrhythmias. 


\subsection{FIGURES AND TABLES}

Table 1 Cardiovascular Data During Cooling and Rewarming.

Values are medians (ranges)

\begin{tabular}{|c|c|c|c|c|c|}
\hline & $\begin{array}{l}\text { Group } 1 \\
\left(37^{0} \mathrm{C}\right)\end{array}$ & $\begin{array}{l}\text { Group } 2 \\
\left(36^{0} \mathrm{C} \text { for }\right. \\
24 \text { hours }) \\
\end{array}$ & $\begin{array}{l}\text { Group } 3 \\
\left(35^{\circ} \mathrm{C} \text { for }\right. \\
24 \text { hours }) \\
\end{array}$ & $\begin{array}{l}\text { Group } 4 \\
\left(34^{0} \mathrm{C} \text { for }\right. \\
24 \text { hours }) \\
\end{array}$ & $\begin{array}{l}\text { Group } 5 \\
\left(34^{0} \mathrm{C} \text { for }\right. \\
48 \text { hours }) \\
\end{array}$ \\
\hline $\begin{array}{l}\text { Heart rate prior to } \\
\text { cooling } \\
\text { (beats/minute) }\end{array}$ & $\begin{array}{c}165 \\
(150-176)\end{array}$ & $\begin{array}{c}170 \\
(132-188)\end{array}$ & $\begin{array}{c}149 \\
(130-214)\end{array}$ & $\begin{array}{c}165 \\
(130-201)\end{array}$ & $\begin{array}{c}168 \\
(126-178)\end{array}$ \\
\hline $\begin{array}{l}\text { Heart rate during } \\
\text { cooling } \\
\text { (beats/minute) }\end{array}$ & $\begin{array}{c}136^{*} \\
(85-200)\end{array}$ & $\begin{array}{l}129 \\
(78-188)\end{array}$ & $\begin{array}{l}103 \\
(68-214)\end{array}$ & $\begin{array}{l}127 \\
(74-201)\end{array}$ & $\begin{array}{c}108 \\
(82-187)\end{array}$ \\
\hline $\begin{array}{l}\text { Heart rate when } \\
\text { rewarmed } \\
\text { (beats/minute) }\end{array}$ & $\begin{array}{l}134 \\
(99-180)\end{array}$ & $\begin{array}{l}120 \\
(82-171)\end{array}$ & $\begin{array}{l}124 \\
(98-188)\end{array}$ & $\begin{array}{c}149 \\
(94-204)\end{array}$ & $\begin{array}{c}132 \\
(99-181)\end{array}$ \\
\hline $\begin{array}{l}\text { MABP prior to } \\
\text { cooling (mmHg) }\end{array}$ & $\begin{array}{c}44 \\
(34-49)\end{array}$ & $\begin{array}{c}36 \\
(25-80)\end{array}$ & $\begin{array}{c}50 \\
(28-70)\end{array}$ & $\begin{array}{c}37 \\
(28-49)\end{array}$ & $\begin{array}{c}42 \\
(35-45)\end{array}$ \\
\hline $\begin{array}{l}\text { MABP during cooling } \\
(\mathrm{mmHg})\end{array}$ & $\begin{array}{c}52 * \\
(34-83)\end{array}$ & $\begin{array}{c}56 \\
(36-90)\end{array}$ & $\begin{array}{c}54 \\
(36-82)\end{array}$ & $\begin{array}{c}51 \\
(27-77)\end{array}$ & $\begin{array}{c}50 \\
(33-87)\end{array}$ \\
\hline $\begin{array}{l}\text { MABP when } \\
\text { rewarmed (mmHg) } \\
\text { Fall in heart rate } \\
\text { during Cooling } \\
\text { (beats/minute) }\end{array}$ & $\begin{array}{c}52 \\
(38-75) \\
\text { Not Cooled }\end{array}$ & $\begin{array}{c}59 \\
(36-81) \\
46 \\
(3-57)\end{array}$ & $\begin{array}{c}53 \\
(35-86) \\
41 \\
(21-105)\end{array}$ & $\begin{array}{c}53 \\
(39-76) \\
46 \\
(21-50)\end{array}$ & $\begin{array}{c}52 \\
(38-73) \\
41 \\
(11-74)\end{array}$ \\
\hline
\end{tabular}

*Denotes patients not cooled.

$\mathrm{MABP}=$ Mean arterial blood pressure 
Table 2 Rectal Temperature and Cardiovascular Data During Cooling and Rewarming

\begin{tabular}{|c|c|c|c|c|c|c|}
\hline Infant & $\begin{array}{l}\text { Median (range) } \\
\text { Rectal } \\
\text { Temperature } \\
\text { during Cooling } \\
\quad\left({ }^{\circ} \mathrm{C}\right)\end{array}$ & $\begin{array}{l}\text { Minimal } \\
\text { Rectal } \\
\text { Temperature } \\
\text { during } \\
\text { Cooling } \\
\quad\left({ }^{\circ} \mathrm{C}\right) \\
\end{array}$ & $\begin{array}{l}\text { Number } \\
\text { of Hours } \\
\text { below } \\
\text { target } \\
\text { Temp }\end{array}$ & $\begin{array}{l}\begin{array}{l}\text { Median } \\
\text { (range) }\end{array} \\
\text { MABP } \\
\text { During } \\
\text { Cooling } \\
\text { (mmHg) }\end{array}$ & $\begin{array}{l}\text { Median } \\
\text { (range) Heart } \\
\text { rate during } \\
\text { cooling } \\
\text { (beats/minute) }\end{array}$ & $\begin{array}{l}\text { Minimum Heart } \\
\text { rate during } \\
\text { cooling } \\
\text { (beats/minute) }\end{array}$ \\
\hline \multicolumn{7}{|c|}{$\begin{array}{l}\text { Group } 2 \\
\text { ( } 36^{\circ} \mathrm{C} \text { for } \\
24 \text { hours) }\end{array}$} \\
\hline 6 & $36.0(33.9-36.4)$ & 33.9 & 4 & $57(40-75)$ & $136(94-188)$ & 94 \\
\hline 7 & $36.0(35.5-36.5)$ & 35.5 & 2 & $51(36-60)$ & $117(93-184)$ & 93 \\
\hline 8 & $36.0(35.5-37.1)$ & 35.5 & 1 & $55(47-70)$ & $136(122-170)$ & 122 \\
\hline 9 & $36.0(35.7-36.2)$ & 35.7 & 1 & $55(37-79)$ & $135(121-179)$ & 121 \\
\hline 10 & $36.0(35.7-36.5)$ & 35.7 & 1 & $68(50-90)$ & $105(78-176)$ & 78 \\
\hline \multicolumn{7}{|c|}{$\begin{array}{l}\text { Group } 3 \\
\left(35^{\circ} \mathrm{C} \text { for }\right. \\
24 \text { hours })\end{array}$} \\
\hline 11 & $35.0(34.4-36.3)$ & 34.4 & 2 & $56(48-76)$ & $97 \quad(90-130)$ & 90 \\
\hline 12 & $35.0(34.0-36.8)$ & 34.0 & 1 & $58(41-74)$ & $80 \quad(68-156)$ & 68 \\
\hline 13 & $35.0(34.0-35.3)$ & 34.0 & 1 & $44(36-55)$ & $140(102-167)$ & 102 \\
\hline 14 & $35.0(34.4-36.6)$ & 34.4 & 2 & $53(39-82)$ & $99(86-214)$ & 86 \\
\hline 15 & $35.0(34.9-36.4)$ & 34.9 & 0 & $58(42-82)$ & $114(94-192)$ & 94 \\
\hline \multicolumn{7}{|c|}{$\begin{array}{l}\text { Group } 4 \\
\left(34^{0} \mathrm{C} \text { for }\right. \\
24 \text { hours) }\end{array}$} \\
\hline 16 & $34.0(33.5-37.2)$ & 33.5 & 2 & $52(27-70)$ & $150(122-201)$ & 122 \\
\hline 17 & $33.9(33.0-35.0)$ & 33.0 & 2 & $55(35-67)$ & $108(95-145)$ & 95 \\
\hline 18 & $34.0(33.8-37.8)$ & 33.8 & 0 & $55(46-77)$ & $82 \quad(74-165)$ & 74 \\
\hline 19 & $34.0(33.7-36.7)$ & 33.7 & 1 & $59(40-70)$ & $123(104-139)$ & 104 \\
\hline 20 & $34.1(33.8-36.0)$ & 33.8 & 0 & $39(29-41)$ & $157(148-198)$ & 148 \\
\hline \multicolumn{7}{|c|}{$\begin{array}{l}\text { Group } 5 \\
\left(34^{0} \mathrm{C} \text { for }\right. \\
\mathbf{4 8} \text { hours) }\end{array}$} \\
\hline 21 & $34.0(33.7-34.0)$ & 33.7 & 1 & $49(35-87)$ & $113(98-178)$ & 98 \\
\hline 22 & $34.0(33.6-36.5)$ & 33.7 & 2 & $51(33-60)$ & $108(85-178)$ & 85 \\
\hline 23 & $33.9(33.8-37.0)$ & 33.8 & 0 & $58(35-86)$ & $115(93-151)$ & 93 \\
\hline 24 & $34.0(33.4-34.3)$ & 33.4 & 6 & $51(39-83)$ & $95 \quad(82-168)$ & 82 \\
\hline 25 & $34.1(33.7-36.8)$ & 33.7 & 1 & $43(37-49)$ & $102(84-155)$ & 84 \\
\hline
\end{tabular}


Figure 1 Persistent Tachycardia During Cooling and Rewarming

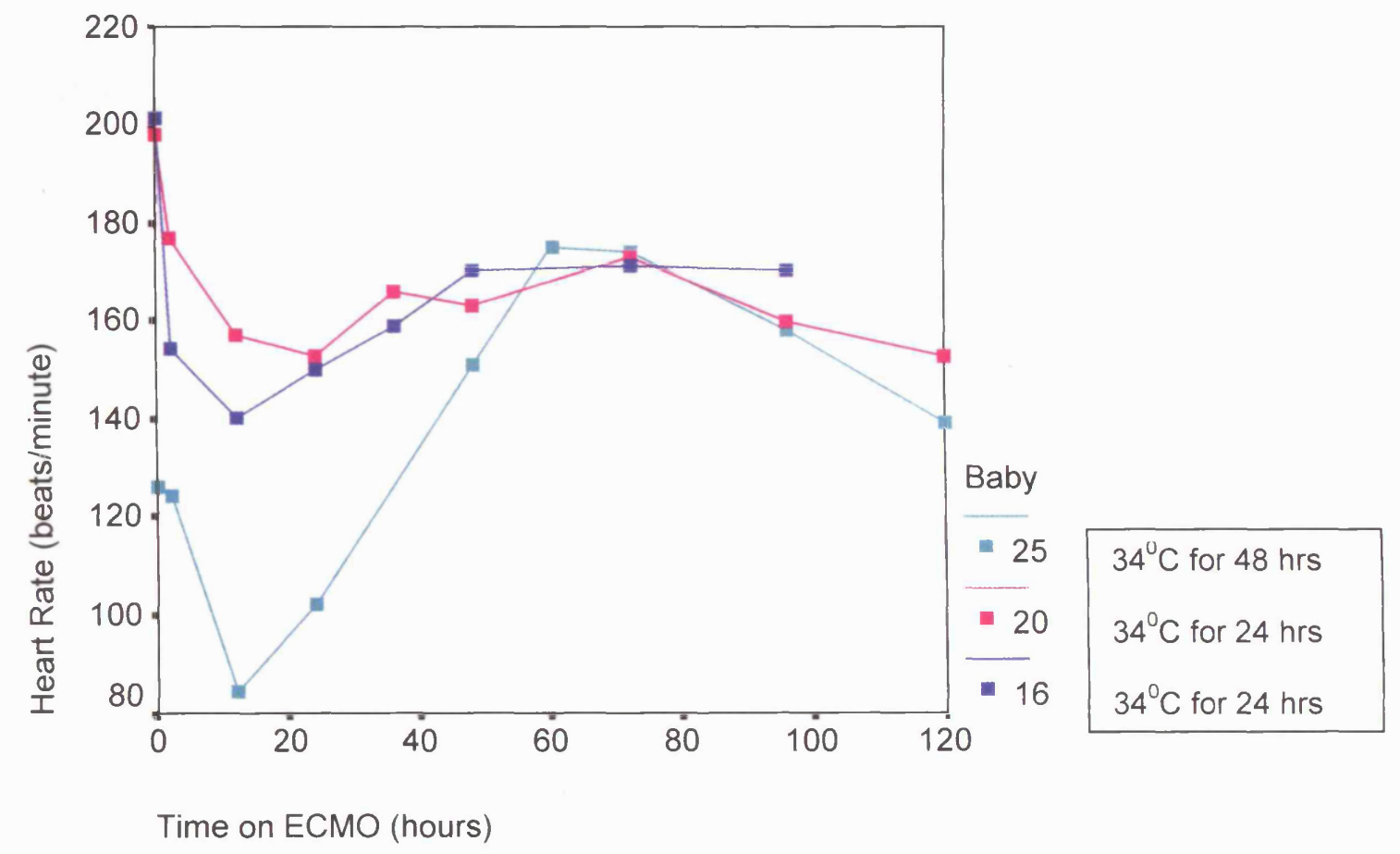

Figure 1 The graph shows the heart rate in beats per minute for baby 25(group 5), and babies 16 and 20 (both in group 4). Each data point represents the heart rate at the time points measured. All three infants had a persistent tachycardia despite cooling to $34^{0} \mathrm{C}$. 


\section{CHAPTER 8}

\section{IMMUNOLOGY AND MILD HYPOTHERMIA}

\section{INTRODUCTION}

In the majority of cases, neonates require ECMO support for reversible cardiopulmonary diseases. Acute severe lung injury and subsequent acute respiratory failure are commonly caused by disorders such as meconium aspiration, sepsis e.g. group B streptococcus and pneumonia. The pulmonary injury is often exacerbated by oxygen toxicity and barotrauma due to mechanical ventilation. ${ }^{345}$ The acute lung injury is thought to be mediated by proinflammatory cytokines such as TNF-alpha,

IL-1 and IL-6. Others such as IL-8 (a potent chemoattractant) may also have a role. ${ }^{346}$ Indeed many of these markers of inflammation have been shown to be increased in blood from infants with respiratory failure who are candidates for neonatal ECMO. ${ }^{347}$ The inflammatory response may be further activated when the infants are placed on ECMO when the infant's blood contacts with the artificial surfaces of the ECMO circuit. $^{348}$

Documented complications of mild hypothermia include immune suppression. The concern therefore of applying mild hypothermia during ECMO is that the immune competence will be affected resulting in increased susceptibility to sepsis.

Measurement of immune function at reduced body temperature during ECMO is problematic since:

1) the presence of the extracorporeal circuit will dilute, and may modulate immune mediators

2) tests of function using cell culture restore the cells to $37^{\circ} \mathrm{C}$ and hence may mask the effects of cooling 
3) investigation of differences in cell populations (lymphocyte phenotypes) will be affected by cells imported in the priming of the circuit with transfused blood. Although there are means of overcoming a number of these problems, the solutions themselves pose significant practical difficulties. For instance, all white cells could be removed from blood given to the patient by filtering all blood products prior to use. However, initiation of ECMO or the need for transfusion is usually performed as an emergency and hence would allow no time for preparation of the blood to be used. Therefore during the pilot study I sought evidence of change in the infants immune competence induced as a result of cooling by:

1) close monitoring of all patients for evidence of infection. Monitoring was done using both routine laboratory tests of blood cultures and C-reactive protein (CRP), chest $\mathrm{X}$ ray and clinical examination.

2) assessing complement activation

3) monitoring inflammatory mediators both pre and during cooling.

\subsection{CYTOKINES IL-6 AND IL-8}

IL-8 is a chemoattractant specific for neutrophils and not monocytes. It is also involved in the inflammatory response and has been shown to cause neutrophilia, neutrophil trapping in the lungs and capillary leak resembling the pathophysiology of the adult respiratory distress syndrome. It is also believed that IL-8 is involved in the regulation of neutrophil transendothelium migration suggesting a significant controlling mechanism for neutrophil-mediated tissue injury.

IL-6 is a cytokine produced by many different cells including monocytes, macrophages, fibroblasts, endothelial cells, mast cells, $\mathrm{T}$ cells and many tumour cell 
lines. The IL (interleukin) -6-type cytokines IL-6, IL-11, LIF (leukaemia inhibitory factor), OSM (oncostatin M), ciliary neurotrophic factor, cardiothrophin-1 and cardiotrophin-like cytokine are important family mediators involved in the regulation of the acute phase response to injury and infection. Besides their functions in inflammation and the immune response, these cytokines play also a crucial role in haematopoiesis, liver and neuronal regeneration, embryonal development and fertility. Dysregulation of IL-6 type cytokine signalling contributes to the onset and maintenance of several diseases, such as rheumatoid arthritis, inflammatory bowel disease, osteoporosis and various types of cancer. IL-6 -type cytokines exert their action via the signal transducers gp (glycoprotein) 130, LIF receptor and OSM receptor leading to the activation of the JAK/STAT (Janus kinase/signal transducer and activator of transcription) and MAPK (mitogen-activated protein kinase) cascades.

\subsection{COMPLEMENT C3a}

Complement is a series of nine plasma proteins which become bound to antigenantibody complexes in a specific sequence (cascade). Binding, or fixation, of complement results in either the lysis or engulfment by phagocytes of the antigen, and can occur by two major routes - the classical and the alternative pathways.

$\mathrm{C} 3 \mathrm{a}$ is produced when $\mathrm{C} 3$ is split by $\mathrm{C} 3$ convertase. $\mathrm{C} 3 \mathrm{a}$ (anaphylatoxin I) is chemotactic for neutrophils and liberates histamine from mast cells. Receptors for this complement component are present on monocytes, neutrophils, mast cells, basophils and eosinophils. 


\subsection{METHODS}

\section{Study Group}

The study included the twenty five patients described in chapters 3 and 4 . The entry criteria and ECMO management are described in chapter 3 . The study groups were identical to those described in chapter 3.

\section{Hypothermic Treatment}

The infants were cooled and rewarmed by adjusting the temperature of the water bath in the extracorporeal circuit as described in chapter 3.

\section{Monitoring and Blood Sample Collection}

Rectal and abdominal skin temperature were monitored continuously. Arterial blood pressure, ECG, respiratory rate and pulse oxymetry were shown continuously on the monitor screen (Hewlett Packard HP Component Monitoring System Model 66S) according to our routine practice. $\mathrm{C}$ reactive protein (CRP) samples were drawn prior to ECMO cannulation ( $\mathrm{t}=0$ hours). In groups $1-4$, following ECMO cannulation further routine CRP samples were drawn from the arterial line at timed intervals of 2 , $12,24,36,48,72,96$ and 120 hours. In group 5 (48 hour cooling), blood samples for CRP were drawn at the same time points except at 60 instead of 36 hours. In addition at the same time intervals, blood was drawn for more specialist tests of immunology: cytokines IL-6, IL-8 and complement C3a. These were centrifuged at 
$3000 \mathrm{RPM}$ at $4^{\circ} \mathrm{C}$ for 15 minutes and stored at $-70^{\circ} \mathrm{C}$ until further analysis.

Investigations for sepsis were conducted and repeated if clinically indicated.

\section{Measurement of IL-6, IL-8 and Complement C3a}

Cytokines IL-6 and IL-8 (Quantikine ${ }^{\circledR}$ R\&D Systems, Inc. MN, USA) and complement C3a (Quidel ${ }^{\circledR}$ CA USA) were analysed by ELISA as per the manufacturer's instructions. The lowest sensitivities of the assays were $6 \mathrm{pg} / \mathrm{ml}$, $10 \mathrm{pg} / \mathrm{ml}$ and $67 \mathrm{ng} / \mathrm{ml}$ respectively. All the assays were performed by Ms Rachel Hodge, (Department of Child Health, Leicester University) who has considerable expertise and experience in ELISA methodology.

\subsection{RESULTS}

\section{Cytokines and Complement}

Cytokines IL-6 and IL-8 followed a similar pattern with the level rising immediately following cannulation and decreasing gradually over the course of the 120 hour study period. In group 5, the rise in the mean IL- 6 and IL-8 level was greater and remained higher than the other groups throughout the study period (see figures 1and 2). There was no impact of cooling on the complement level C3a (see figure 3). 


\section{C-Reactive Protein (CRP)}

In all groups, there was a rise in the CRP following cannulation for ECMO. There was no suppression of CRP with cooling (see Fig 4).

\subsection{DISCUSSION}

Data from patients exposed to more profound hypothermia suggest that immune function is impaired at these low temperatures but no data are available in relation to mild hypothermia. ${ }^{349}$

In normothermic studies of blood activation during ECMO, Plotz et $\mathrm{al}^{329}$ reported a rise in C3a levels on initiation of ECMO. The initial activation pattern subsided by 24 hours. In a study of neutrophil and cytokine activation with neonatal ECMO, Fortenberry et $\mathrm{al}^{347}$ found that the serum levels of IL-6, were significantly elevated prior to ECMO. The IL-6 levels remained elevated for the first 12 hours of ECMO but did not increase further. IL-8 levels were elevated pre-ECMO but became significantly elevated 15 minutes after initiation of ECMO. The IL-8 levels remained elevated for the first 24 hours of ECMO and then gradually decreased. During mild hypothermia, I observed similar patterns of activation of IL-6, IL-8 and C3a. Also, there was no significant impact of mild hypothermia on C-reactive protein.

\subsection{CONCLUSIONS}

There was no significant impact on those parts of the immune system that could be measured comparing cooled infants to those receiving normothermic ECMO. 


\subsection{FIGURES AND TABLES}

Figure 1 Cytokines IL6 and IL8 for Study Groups
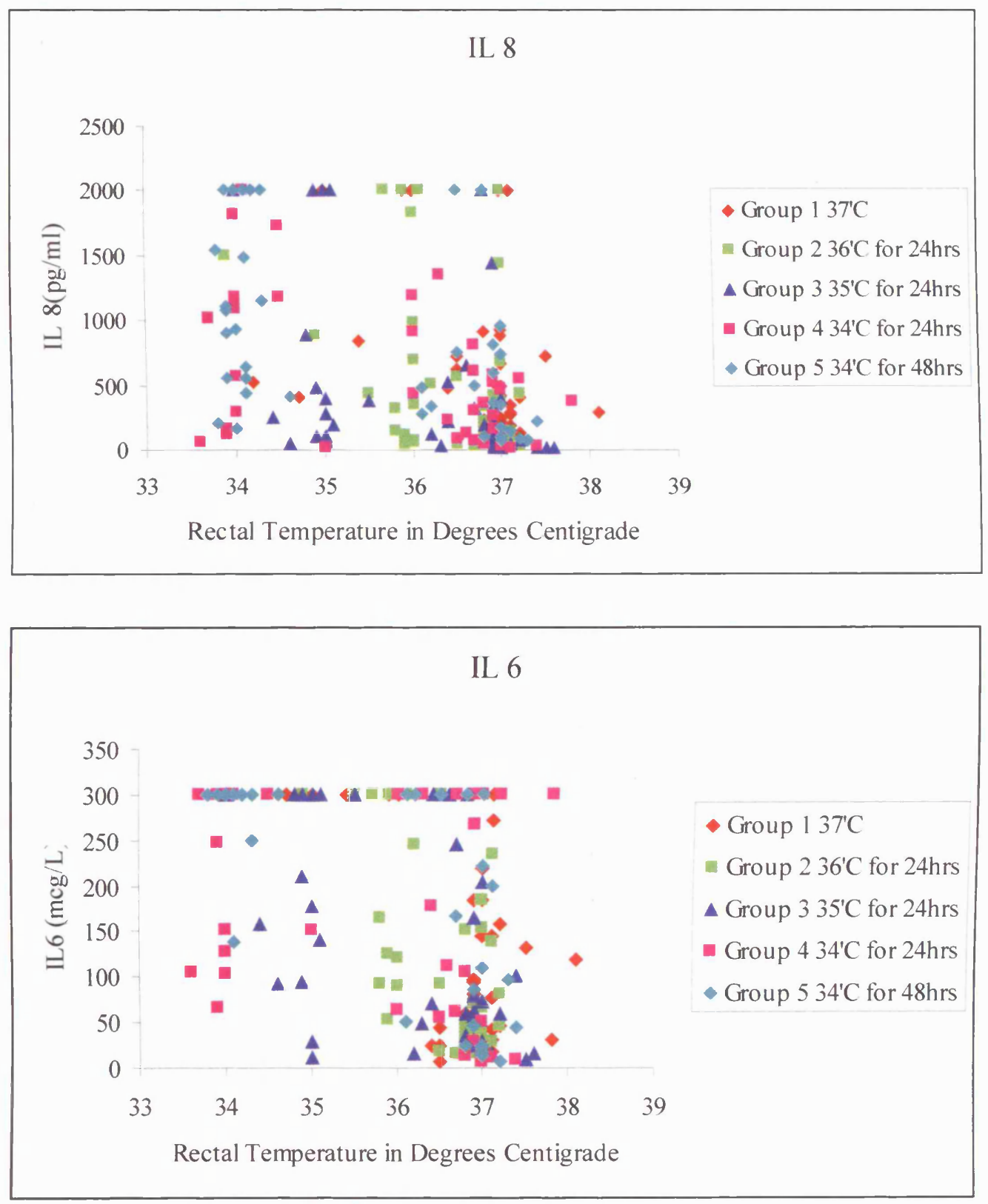

Figure 1 Scatter plot showing the cytokines IL8 and IL6 values for each individual patient in the five temperature groups. The graphs display the values for IL8 and IL6 for the control group also during the cooling period ( 24 or 48 hours) and when rewarmed to $37^{\circ} \mathrm{C}$. 
Figure 2 Complement $\mathrm{C} 3 \mathrm{a}$

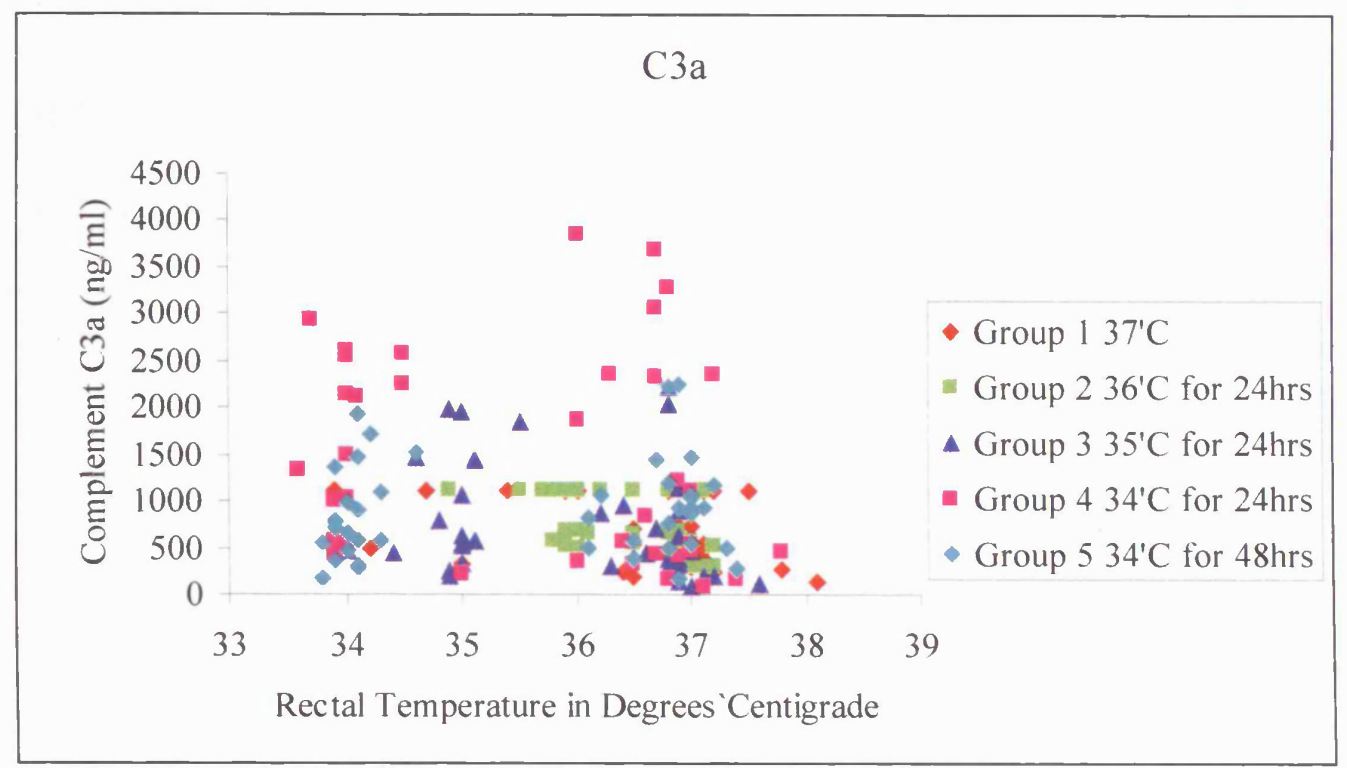

Figure 2 Scatter plot showing complement $\mathrm{C} 3$ a values for each individual patient in the five temperature groups. The graphs display the values for complement $\mathrm{C} 3 \mathrm{a}$ for the control group also during the cooling period ( 24 or 48 hours) and when rewarmed to $37^{0} \mathrm{C}$.

Figure $3 \mathrm{C}$ - Reactive Protein

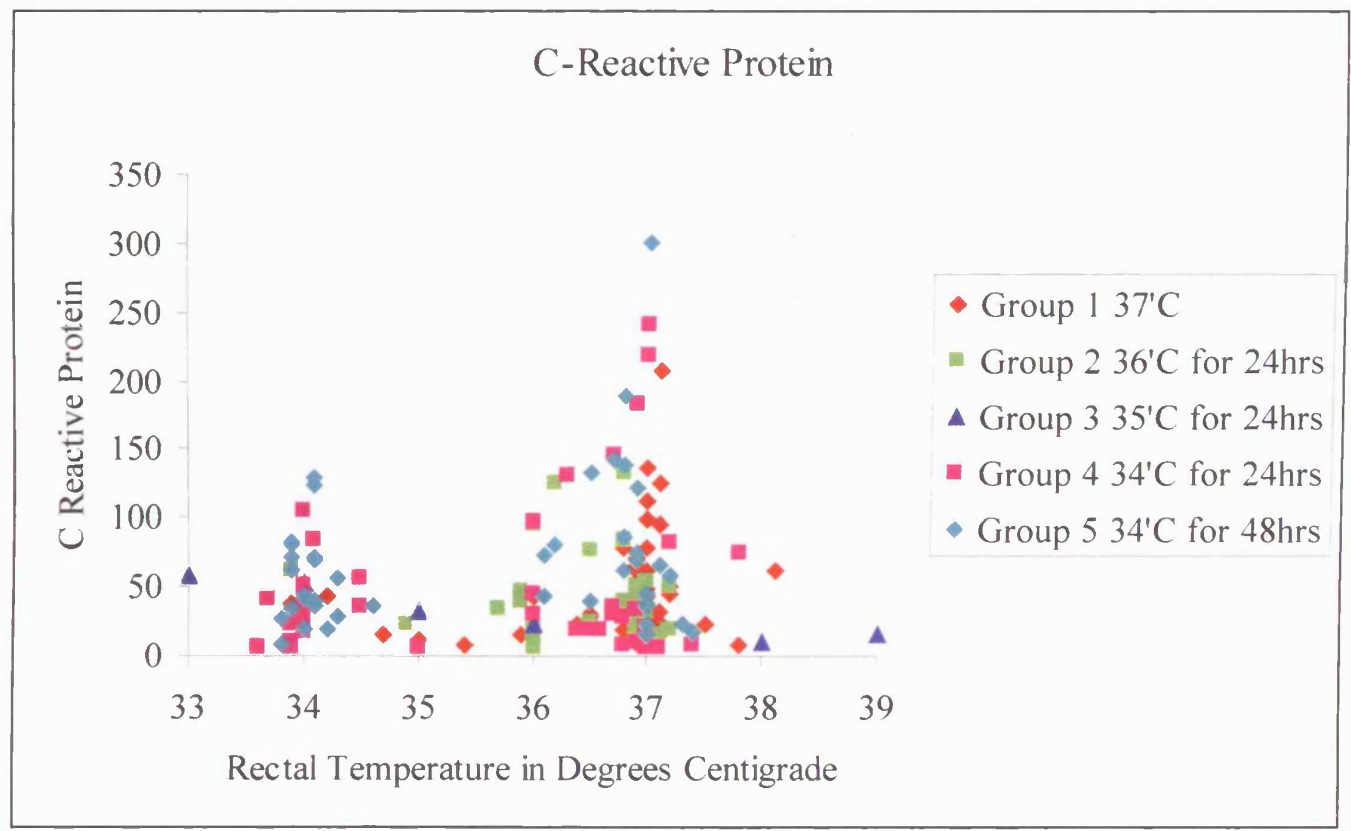

Figure 2 Scatter plot showing the C-reactive protein values for each individual patient in the five temperature groups. The graphs display the values for C-reactive protein for the control group also during the cooling period ( 24 or 48 hours) and when rewarmed to $37^{\circ} \mathrm{C}$. 


\section{CHAPTER 9}

\section{THE INFLUENCE OF TEMPERATURE ON THE AMPLITUDE}

\section{INTEGRATED ELECTROENCEPHALOGRAM DURING NEONATAL}

ECMO

\section{INTRODUCTION}

Neonates who receive extracorporeal membrane oxygenation (ECMO) are at high risk of hypoxic -ischaemic brain injury. However, clinical neurological assessment during ECMO is complicated by the severity of illness and the use of sedative drugs.

Previously, serial standard electroencephalograhpy (EEG) has been used to predict the neurological outcome for neonatal ECMO. The most significant EEG abnormalities in terms of predicting poor prognosis were two or more EEG recordings showing either burst suppression or seizures. Single standard EEG recordings were of no prognostic value. ${ }^{350}$ However, standard EEG requires considerable expertise for recording and analysis and may not be readily accessible in most general hospitals.

An alternative technique is the amplitude-integrated electroencephalogram (aEEG) recorded using a cerebral function monitor (CFM). A single-channel EEG signal is obtained from biparietal electrodes; frequencies $<2 \mathrm{~Hz}$ and $>15 \mathrm{~Hz}$ are filtered selectively, and the amplitude-integrated signal is recorded onto an integral printer. The aEEG has has been shown to be highly predictive of neurological outcome in neonatal encephalopathy. ${ }^{351}$ Also, the aEEG correlates well with the standard EEG in terms of agreement in background activity and prognostic evaluation. ${ }^{352-353}$ The use of aEEG during neonatal ECMO has not been previously reported. 
Mild hypothermia appears to be a promising means of neuroprotection. Evidence from studies in animals have shown that a reduction in the body temperature by $3^{\circ} \mathrm{C}$ or $4^{0} \mathrm{C}$ after an hypoxic ischaemic insult preserves cerebral energy metabolism, reduces cytotoxic oedema, and improves histological and behavioural outcome. ${ }^{3-6}$ In adults, mild cooling for 24-48 hours following cardiac arrest due to ventricular arrhythmia significantly improves survival and neurological outcome. ${ }^{354}$ In previous studies of mild hypothermia in neonates following hypoxic-ischaemic cerebral injury, the aEEG has been used as a tool to select infants most severely affected who might benefit from cooling. ${ }^{275.344}$ In these studies, the aEEG was assessed prior to cooling but the influence of mild hypothermia on the aEEG has not been reported. Although deep hypothermia to less than $30^{\circ} \mathrm{C}$ causes suppression of the $\mathrm{EEG}^{355-356}$ the effect of prolonged induced mild hypothermia on the EEG is uncertain.

During a pilot study of mild hypothermia in neonates receiving ECMO, I explored the use of the aEEG to address the following questions:

1) What is the effect of mild hypothermia on the aEEG?

2) What are the changes on the aEEG during neonatal ECMO?

\subsection{METHODS}

The study group included twenty two of the patients described in chapters 3 and 4 . I have also included the aEEG data of four infants who were recruited into the study but later excluded from the final data analysis. All four infants were cooled and rewarmed as per the protocol and the decision to exclude them from the study was based on problems of temperature control on day 3 to 5 of the pilot study. To examine the 
influence of temperature on the aEEG, I compared the aEEG amplitude during the last six hours of cooling with the first six hours once the infants were rewarmed. Since all four of the infants were still in the protocol at this stage, I have included their aEEG data in this analysis. The four traces also provided further data on the aEEG changes during neonatal ECMO. The entry criteria and ECMO management described in chapter 3 were identical for the study group.

All infants were sedated using infusions of morphine and midazolam. Bolus doses of ketamine were given for procedures performed during ECMO.

\section{Study Group}

Group 1: Six neonates maintained at $37^{\circ} \mathrm{C}$ core temperature throughout the first five days of ECMO (control group)

Group 2: Four neonates maintained at $36^{\circ} \mathrm{C}$ core temperature for the first 24 hours of ECMO, then rewarmed to $37^{0} \mathrm{C}$ for the next four days.

Group 3: Five neonates maintained at $35^{\circ} \mathrm{C}$ core temperature for the first 24 hours of ECMO, then rewarmed to $37^{\circ} \mathrm{C}$ for the next four days.

Group 4: Six neonates maintained at $34^{\circ} \mathrm{C}$ core temperature for the first 24 hours of ECMO, then rewarmed to $37^{\circ} \mathrm{C}$ for the next four days.

Group 5: Five neonates maintained at $34^{\circ} \mathrm{C}$ core temperature for the first 48 hours of ECMO, then rewarmed to $37^{\circ} \mathrm{C}$ for the next three days. 


\section{Hypothermic Treatment}

The infants were cooled and rewarmed by adjusting the temperature of the water bath in the extracorporeal circuit as described in chapter 3.

\section{Problems with the Amplitude-Integrated Electroencephalogram}

The amplitude -integrated electroencephalogram (aEEG) had not been used previously during neonatal ECMO at the Glenfield Hospital. I was given brief training on the aEEG by Dr Denis Azzopardi at the Hammersmith Hospital, London. One concern of using the aEEG during ECMO was the potential for bleeding from the scalp needle electrode sites in a heparinised patient. I explored the use of the alternative surface disc electrodes. I found initial difficulty in obtaining a low impedence trace using the electrode adhesive supplied by the aEEG manufacturer. I sought the advice of the EEG technicians at the Department of Electrophysiology at the Leicester Royal Infirmary. Using the technique they use to apply surface electrodes for standard EEG's I was able (in the majority of cases) to obtain a low impedence trace. In the patients where I had to resort to using needle electrodes, there were no problems of bleeding.

At the outset of the study, I had aimed to obtain a short $10-15$ min aEEG recording prior to ECMO cannulation to allow comparison of the aEEG traces pre and post commencement of ECMO. In reality this proved impossible in the majority of cases for the following reasons: the time at the referral centre was limited by the need to transfer to infant as quickly as possible for ECMO. I found that the majority of my time at the referral centre was used to speak to the parents about ECMO and the 
research study. This usually left insufficient time to set up and record the aEEG. Where I attempted to record the aEEG, it was often difficult to obtain a good quality aEEG trace as the patient was frequently disturbed or moved by the ECMO team who were stabilising the infant prior to transfer. Once the infants arrived at the Glenfield Hospital, the patients mostly required immediate cannulation for ECMO which meant that an aEEG could not obtained until the patient was stabilised on ECMO. In the final analysis I used the aEEG recordings obtained following ECMO cannulation.

\section{Amplitude-Integrated Electroencephalogram}

The aEEG was recorded in all infants with a Lectromed CFM (CFM 5330, Lectromed UK, Herts, UK) from biparietal needle or surface disc electrodes and displayed on the integral printer at $6 \mathrm{~cm} /$ hour. The aEEG was calibrated before each recording. The recording was commenced following cannulation for ECMO and recorded continuously during the first five days of ECMO. The aEEG was classified as normal, moderately or severely suppressed as previously reported (see Figs $1 \& 2$ ). ${ }^{351}$ A normal aEEG consisted of a band of activity with an upper margin $>10 \mu \mathrm{V}$ and lower margin $>5 \mu \mathrm{V}$. A moderately suppressed aEEG was defined as an upper margin $>$ $10 \mu \mathrm{V}$ and a lower margin $\leq 5 \mu \mathrm{V}$. A severely suppressed trace defined as a upper $\operatorname{margin}<10 \mu \mathrm{V}$.

Seizures were defined as a sudden rise in voltage usually accompanied by a narrowing of the aEEG amplitude. 
Measurement of Effect of Mild Hypothermia

The median and range values for the upper and lower margin voltages of the aEEG trace were measured for groups 2 - 5 for the final six hours of the cooling period and compared with the values obtained during the first six hours once the infants were rewarmed (see Figure 3).

\section{Monitoring}

Rectal and abdominal skin temperatures were monitored continuously. Arterial blood pressure, ECG, respiratory rate and pulse oxymetry were shown continuously on the monitor screen (Hewlett Packard HP Component Monitoring System Model 66S) according to our routine practice. 


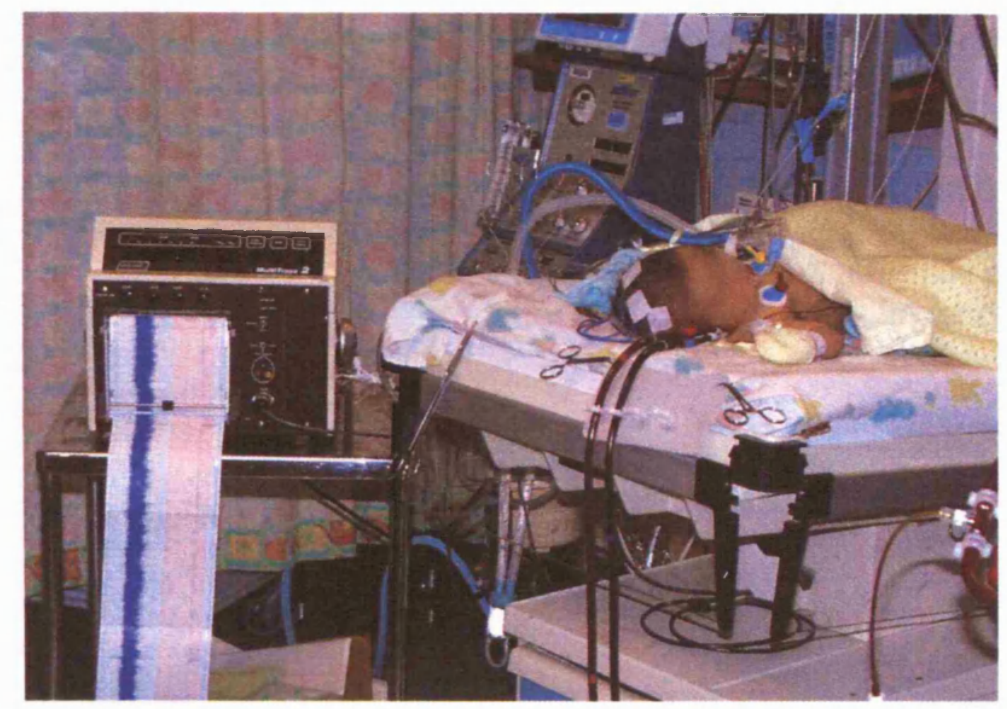

Figure 1. Measurement of aEEG

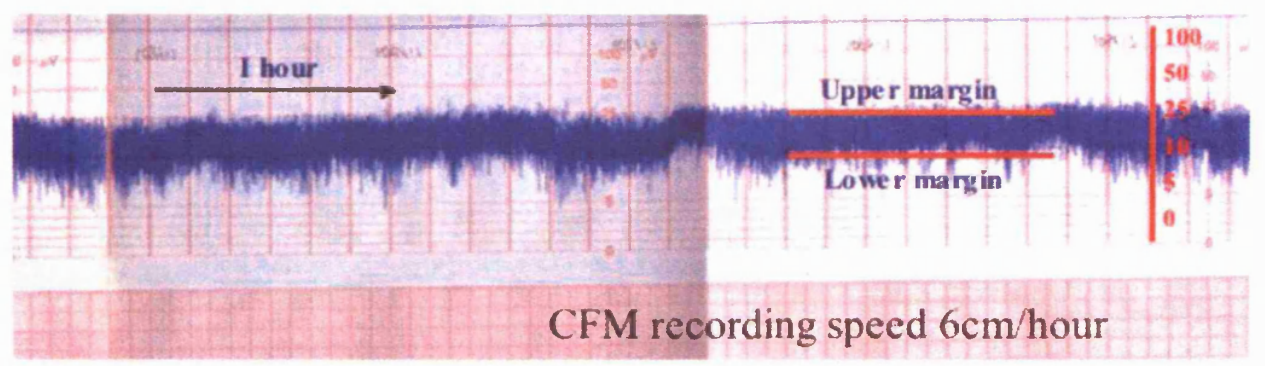


Figure 2 Classification of aEEG Traces

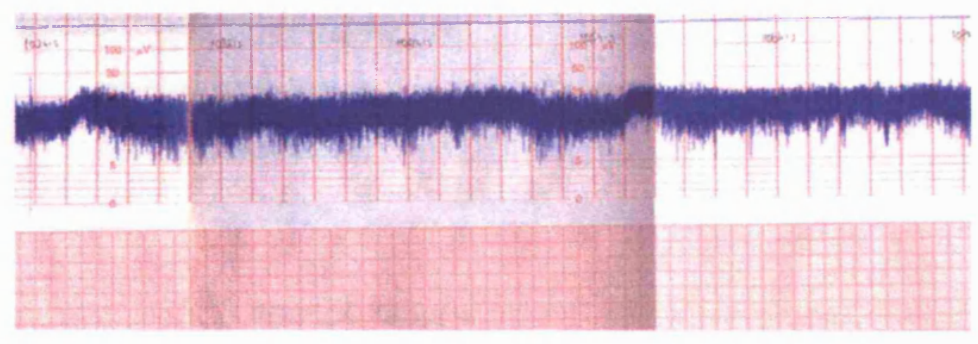

\section{Normal}

upper margin $>10 \mu$ volts

lower margin $>5 \mu$ volts

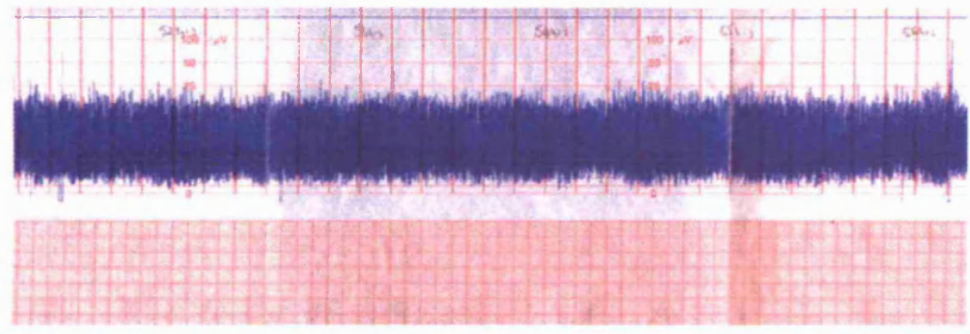

Moderately Suppressed

upper margin $>10 \mu$ volts

lower margin $\leq 5 \mu$ volts

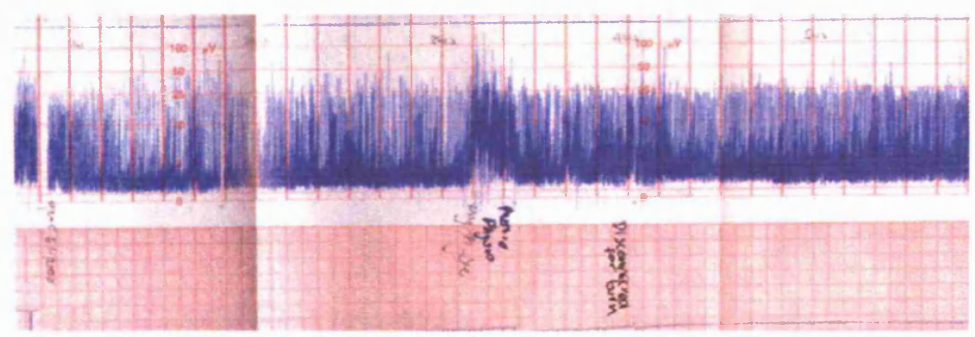

Severely Suppressed

upper margin $<10 \mu$ volts

lower margin $<5 \mu$ volts 
Figure 3 Measurement of the Effect of Mild Hypothermia

\section{Group}

$36^{\circ} \mathrm{C}$ for 24 hours

2

Cooling 33 hrs $^{*}$ Rewarmed

$35^{\circ} \mathrm{C}$ for 24 hours

3

Cooling $41 \mathrm{hrs}^{*} \quad$ Rewarmed

$34^{\circ} \mathrm{C}$ for 24 hours



*Median values

Arrowes indicate when aEEG was sampled i.e. final $6 \mathrm{hrs}$ of cooling and first $6 \mathrm{hrs}$ when rewarmed 


\subsection{STATISTICAL ANALYSIS}

Pre ECMO data was compared by the Kruskal-Wallis test. Comparisons between groups were also made using the Kruskal-Wallis test. A p value $<0.005$ was considered to be statistically significant.

\subsection{RESULTS}

\section{Patient Characteristics}

The clinical characteristics of the patients included in the study are summarised in table 1. No significant difference was found between the five study groups in terms of pre-ECMO demographic data. In those patients not surviving to discharge (table1) the causes of death were as follows: - In group 2, baby 8 had a congenital diaphragmatic hernia which was repaired on ECMO and the infant successfully extubated prior to discharge. The infant died age 3 months old from respiratory failure. Also in group 2 , intensive care was withdrawn in patient 9 due to inoperable total anomalous pulmonary venous drainage (TAPVD). In group 4, patient 16 had a inborn error of metabolism (myophosphorylase deficiency), patient 17 suffered a large posterior fossa cerebral haemorrhage on day 11 of ECMO, patient 18 had a congenital diaphragmatic hernia and intensive care was withdrawn on day 20 of life due to intractable pulmonary hypertension and patient 20 developed multiorgan failure secondary to presumed pertussis sepsis and died on day 8 of ECMO. 


\section{The Application of aEEG During ECMO}

In twenty two (85\%) patients, the CFM recording was commenced within the first six hours following ECMO cannulation. There were no problems of electrical interference from the surrounding ECMO equipment disrupting the aEEG trace. Recordings of 120 hours were obtained in $9(35 \%)$ patients, $90-114$ hours in $9(35 \%)$ patients, $66-84$ hours in $5(19 \%)$ patients and 48 hours in $1(4 \%)$ patients. In $2(7 \%)$ patients, there was limited recording of 18 hours due to poor electrode contact. In the majority of infants, it was possible to obtain a low impedance trace using surface electrodes. However, where needle electrodes were placed there were no problems of bleeding from the needle site.

Seven $(30 \%)$ of the twenty six traces were excluded from the analysis of the effect of mild hypothermia due to either no recording or poor quality trace during the selected cooling and rewarming times.

\section{The Application of Hypothermia}

Cooling was initiated immediately following cannulation for ECMO. Using the extracorporeal circuit, accurate control of the core temperature was possible as illustrated in figure 4.

Cooling was achieved in groups $2-5$ by a median of 6 hours (range $0-12$ hours) At the end of cooling the patients were rewarmed to $37^{\circ} \mathrm{C}$ at rate of $0.5^{\circ} \mathrm{C}$ per hour. The median (range) times to rewarming for groups 2 - 5 were 36(36-42), 42(42-48), 57(42-66) and 72(60-72) hours after starting ECMO. 


\section{The Effect of Mild Hypothermia on aEEG amplitude}

$19(73 \%)$ traces were suitable for analysis. There was no significant difference in the upper and lower margin voltage values during cooling and after rewarming (Tables 3 and 4). The mean rectal temperature during cooling and after rewarming at the time aEEG measurement is shown in (Table 4)

\section{aEEG Traces}

Twenty six traces were obtained of which $16(62 \%)$ were normal or mildly abnormal throughout and six (23\%) were intermittently moderately abnormal for median (range) $54(24,78)$ hours of trace. One aEEG trace was severely suppressed for 102 hours. Three (11\%) traces had prolonged period of seizure activity for 48,24 and 48 hours of the trace respectively and in two infants, these were not associated with clinical manifestation of seizures. Details of the aEEG traces are shown in table 2. In 18 traces, the aEEG amplitude increased during the first 12 hours following cannulation for ECMO. aEEG amplitude was reduced immediately following bolus doses of sedation but the amplitude returned to previous levels within one hour. Continuous infusion of morphine and midazolam did not appear to affect the aEEG amplitude, since the aEEG amplitude did not alter when the infusions were commenced or discontinued.

\section{Changes in aEEG with Acute Neurological Complications}

Figure 4, illustrates the changes in aEEG with acute neurological complications. 
Trace A demonstrates a sudden change of the aEEG trace to continuous seizures at 35 hours on ECMO. At this time the cranial ultrasound scan was normal but the infant developed a large left cerebral haemorrhage 24 hours later. Trace B shows frequent seizure activity on the aEEG starting twenty four hours before the seizures became clinically apparent.

\subsection{DISCUSSION}

In this study I found no influence of mild hypothermia to $34^{\circ} \mathrm{C}$ on the aEEG. The aEEG could be recorded for several hours in all infants and was helpful in identifying complications during treatment with ECMO.

\section{The Effect of Mild Hypothermia on aEEG Amplitude}

In previous pilot studies of mild hypothermia as a neuroprotective strategy for hypoxic ischaemic encephalopathy, the aEEG has been used as tool to select infants who might benefit from cooling. None of the previous studies have examined the effect of mild hypothermia on the aEEG amplitude. I found no significant change in the aEEG amplitude during cooling and when the infants were rewarmed to $37^{\circ} \mathrm{C}$. This study thus demonstrates that the aEEG can be reliably used during treatment with mild hypothermia to $34^{0} \mathrm{C}$. However, deeper hypothermia is likely to affect the aEEG because of the known effect of deep hypothermia, below about $30^{\circ} \mathrm{C}{ }^{336}$ on the EEG, but this could not be examined in this study as the core temperature was not allowed to fall below $34^{\circ} \mathrm{C}$. 


\section{The amplitude integrated electroencephalogram (aEEG) during ECMO}

Standard electroencephalography (EEG) has been used to predict the neurological outcome for neonatal ECMO. In a series of 119 term or near term neonates treated by venoarterial ECMO, Graziani et al ${ }^{350}$ studied the prognostic significance of EEG's recorded serially at 2 to 4 day intervals during the acute phase of ECMO treatment. Infants who had two or more recordings of burst suppression or electrographic seizures when compared to infants without such EEG abnormalities, had a significantly increased odds ratio for a poor prognosis. Single EEG recordings were of no prognostic value.

A disadvantage of the standard EEG is that it requires considerable expertise both in application and interpretation. In many institutions EEG, particularly serial or continuous EEG recordings are not readily accessible and hence I explored in this study the amplitude integrated electroencephalogram (aEEG) as an alternative method of acute neurological assessment of neonates receiving ECMO.

The aEEG recorded using a cerebral function monitor has been shown to be highly predictive of neurological outcome in neonatal encephalopathy. ${ }^{351}$ To my knowledge there have been no previous reports of the use of aEEG during neonatal ECMO. I did not observe any complications related to the aEEG. In the majority of cases, a low impedence trace was obtained using surface electrodes except in some cases of scalp oedema. Where needle electrodes were used there were no problems of bleeding from the needle site or any injury to the scalp. In all cases the aEEG recording was commenced without difficulty following cannulation for ECMO. However, attention was required for the first one to two hours of the aEEG recording to ensure that the impedance on the trace was not increasing. This change in impedance was usually due 
to the need to move or disturb the infant for essential treatment or investigations during the acute phase of the ECMO course. Once the patient was stabilised then a continuous recording was possible, with recordings of between 90 to 120 hours obtained in $70 \%$ of patients.

I observed an increase in aEEG amplitude during the first 12 hours following cannulation for ECMO in many infants, which may be due to the improvement in oxygenation following cannulation for ECMO.

The infants enrolled in the study were referred for ECMO because of severe acute respiratory and/or cardiac failure. Clearly, the risk of hypoxic-ischaemic brain injury in this situation is not entirely analogous to neonatal hypoxic ischaemic encephalopathy. However, in my study I obtained aEEG traces which could be classified as normal, moderately and severely suppressed as described by previous authors. $^{351}$

The majority of the traces $(85 \%)$ were either normal or mildly abnormal throughout the recording. I observed that two infants with a normal aEEG and two with a moderately suppressed aEEG died. Only in one of these cases death was related to a neurological cause. This infant developed a large posterior fossa haemorrhage on day 11 of ECMO, five days after completion of the study period and discontinuation of aEEG monitoring. Two infants with severely abnormal aEEGs with periods of continuous seizure activity died. In both cases, the infants were encephalopathic and one developed a left sided cerebral haemorrhage. However, due to the limitation of the design of this study it was not possible to correlate the aEEG with neurological outcome. Further investigations are required to clarify the predictive power of aEEG in neonatal ECMO. 
Neonates who receive ECMO represent a group at high risk of hypoxic-ischaemic cerebral injury. A particular concern during neonatal ECMO is the development of intracranial haemorrhage (ICH) therefore neurological assessment is important for clinical management. Unfortunately, clinical assessment is complicated by the severity of illness and the use of sedative and paralysing agents. A retrospective analysis reported by De Sanctis et $\mathrm{al}^{357}$ found that no clinical parameter helped to distinguish infants on ECMO who developed ICH from those who did not. Most ECMO centres rely on cranial ultrasound (US) scans as an adjuvant to clinical assessment during ECMO. Cranial US is used to identify ICH before ECMO, and to detect new or evolving haemorrhage in the course of ECMO, but the appearance of ICH on US may be delayed. Continuous aEEG recording enables detection of changes in aEEG amplitude that may alert the clinician to complications such as ICH. Two infants had acute changes in the aEEG before cranial US changes of ICH or clinical manifestation of seizures. Seizures may be difficult to recognise clinically if infants are heavily sedated and ventilated. Although accurate and complete detection of seizures by the aEEG is difficult, newer cerebral function monitors that also display the standard EEG facilitate diagnosis of seizures and the assessment of response to anticonvulsant therapies. Although cranial US is a sensitive tool for major ICH, it may not detect other clinically significant injuries. ${ }^{358}$ Previous studies have shown that even when the cranial US is normal, follow-up neuroimaging scans and developmental assessment are essential in predicting neurological outcome in ECMO survivors. $^{359}$ 


\subsection{CONCLUSIONS}

I have demonstrated that mild hypothermia to $34^{\circ} \mathrm{C}$ does not influence the amplitude of the aEEG. Therefore, the aEEG can be reliably used during mild systemic hypothermia. I found that continuous aEEG recording was possible during neonatal ECMO and allowed detection of acute neurological complications. Further studies are required to determine the predictive power of the aEEG for neurodevelopmental outcome following neonatal ECMO. 


\subsection{FIGURES AND TABLES}

Table 1 Summary of Demographic Data for Study Groups

\begin{tabular}{|c|c|c|c|c|c|c|c|}
\hline & $\begin{array}{l}\text { Group } 1 \\
\left(37^{0} \mathrm{c}\right)\end{array}$ & $\begin{array}{l}\text { Group } 2 \\
\left(36^{0} \mathrm{c} \text { for }\right. \\
24 \text { hours }) \\
\end{array}$ & $\begin{array}{l}\text { Group } 3 \\
\left(35^{0} \mathrm{c} \text { for }\right. \\
24 \text { hours) }\end{array}$ & $\begin{array}{l}\text { Group } 4 \\
\left(34^{0} \mathrm{c} \text { for }\right. \\
24 \text { hours }) \\
\end{array}$ & $\begin{array}{l}\text { Group } 5 \\
\left(34^{0} \mathrm{c} \text { for }\right. \\
48 \text { hours }) \\
\end{array}$ & $\begin{array}{l}\text { Chi- } \\
\text { squared* }\end{array}$ & $\mathrm{p}$ \\
\hline $\begin{array}{l}\text { Gestation in } \\
\text { Weeks }\end{array}$ & $\begin{array}{c}40 \\
(33-40)\end{array}$ & $\begin{array}{c}39 \\
(38-40)\end{array}$ & $\begin{array}{l}40 \\
(39-41)\end{array}$ & $\begin{array}{c}38 \\
(37-40)\end{array}$ & $\begin{array}{c}40 \\
(39-41)\end{array}$ & 8.08 & 0.09 \\
\hline $\begin{array}{l}\text { Age at ECMO } \\
\text { in hours }\end{array}$ & $\begin{array}{c}24 \\
(10-26)\end{array}$ & $\begin{array}{c}107 \\
(26-432)\end{array}$ & $\begin{array}{c}25 \\
(16-312)\end{array}$ & $\begin{array}{c}39 \\
(6-384)\end{array}$ & $\begin{array}{c}22 \\
(12-26)\end{array}$ & 6.93 & 0.14 \\
\hline $\begin{array}{l}\text { Birth Weight } \\
\quad(\mathrm{Kg})\end{array}$ & $\begin{array}{c}3.5 \\
(2.4-4.0)\end{array}$ & $\begin{array}{c}3.5 \\
(3.2-4.3)\end{array}$ & $\begin{array}{c}3.4 \\
(2.8-4.2)\end{array}$ & $\begin{array}{l}3.7 \\
(2.7-4.4)\end{array}$ & $\begin{array}{c}3.4 \\
(3.1-4.3)\end{array}$ & 0.99 & 0.91 \\
\hline Males & 5 & 4 & 2 & 3 & 4 & & \\
\hline $\begin{array}{l}\text { Apgar Score } \\
1 \text { minute }\end{array}$ & $\begin{array}{c}7 \\
(5-8)\end{array}$ & $\begin{array}{c}6 \\
(1-9)\end{array}$ & $\begin{array}{c}6 \\
(4-9)\end{array}$ & $\begin{array}{c}9 \\
(6-10)\end{array}$ & $\begin{array}{c}5 \\
(4-9)\end{array}$ & 7.23 & 0.12 \\
\hline $\begin{array}{l}\text { Apgar Score } \\
5 \text { minutes }\end{array}$ & $\begin{array}{c}9 \\
(7-10)\end{array}$ & $\begin{array}{c}7 \\
(5-10)\end{array}$ & $\begin{array}{c}8 \\
(6-9)\end{array}$ & $\begin{array}{l}10 \\
(7-10)\end{array}$ & $\begin{array}{l}8 \\
(6-10)\end{array}$ & 4.22 & 0.38 \\
\hline pH Pre-ECMO & $\begin{array}{c}7.2 \\
(7.0-7.5)\end{array}$ & $\begin{array}{c}7.2 \\
(7.0-7.4)\end{array}$ & $\begin{array}{c}7.3 \\
(7.0-7.7)\end{array}$ & $\begin{array}{c}7.3 \\
(7.0-7.3)\end{array}$ & $\begin{array}{c}7.2 \\
(7.0-7.4)\end{array}$ & 1.17 & 0.88 \\
\hline $\begin{array}{l}\text { Oxygenation } \\
\text { Index }\end{array}$ & $\begin{array}{c}46 \\
(35-63)\end{array}$ & $\begin{array}{c}51 \\
(27-89)\end{array}$ & $\begin{array}{c}54 \\
(28-90)\end{array}$ & $\begin{array}{c}32 \\
(20-52)\end{array}$ & $\begin{array}{c}40 \\
(31-55)\end{array}$ & 4.04 & 0.40 \\
\hline $\begin{array}{l}\text { Number of Deaths } \\
\text { Primary Diagnose }\end{array}$ & 0 & 2 & 0 & 4 & 0 & & \\
\hline $\begin{array}{l}\text { MAS/PPHN } \\
\text { Sepsis } \\
\text { RDS }\end{array}$ & $\begin{array}{l}5 \\
1\end{array}$ & 2 & 4 & $\begin{array}{l}1 \\
1 \\
1\end{array}$ & $\begin{array}{l}4 \\
1\end{array}$ & & \\
\hline $\begin{array}{l}\text { Congenital } \\
\text { Diaphragmatic } \\
\text { Hernia (CDH) } \\
\text { TAPVD } \dagger \\
\text { Inborn Error of } \\
\text { Metabolism } \ddagger\end{array}$ & & 1 & 1 & 2 & & & \\
\hline
\end{tabular}

*Comparison of five groups by Kruskal-Wallis test $(\mathrm{df}=4)$

$\uparrow$ Total anomalous pulmonary venous drainage $¥$ Myophosphorylase deficiency.

values shown are median (range) 
Figure 4 Median Core Temperature for Study Groups

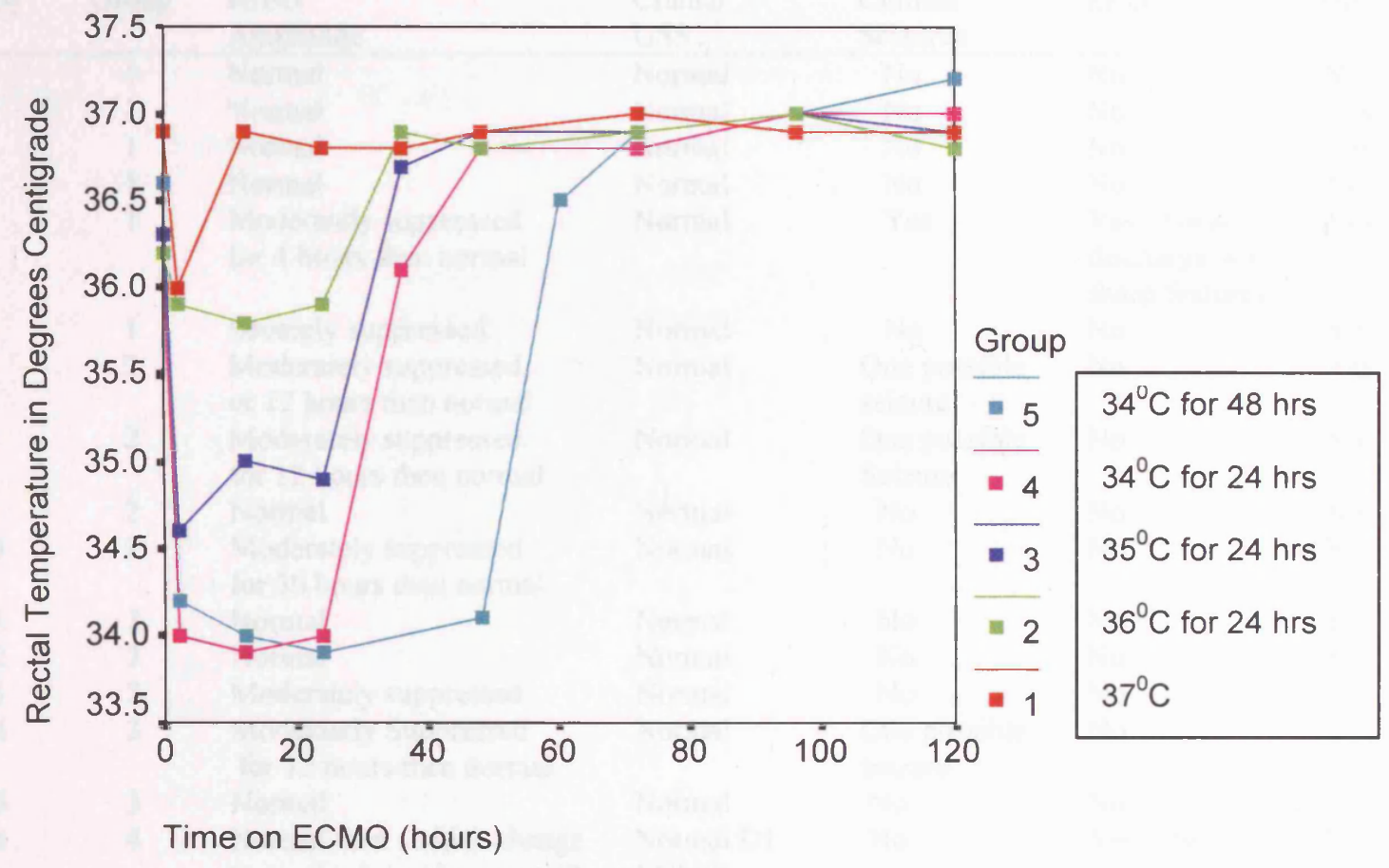


Table 2 Summary of aEEG Traces

\begin{tabular}{|c|c|c|c|c|c|c|}
\hline Baby & Group & $\begin{array}{l}\text { aEEG } \\
\text { Amplitude }\end{array}$ & $\begin{array}{l}\text { Cranial } \\
\text { USS }\end{array}$ & $\begin{array}{l}\text { Clinical } \\
\text { Seizures }\end{array}$ & EEG & Survived \\
\hline 1 & 1 & Normal & Normal & No & No & Yes \\
\hline 2 & 1 & Normal & Normal & No & No & Yes \\
\hline 3 & 1 & Normal & Normal & No & No & Yes \\
\hline 4 & 1 & Normal & Normal & No & No & Yes \\
\hline 5 & 1 & $\begin{array}{l}\text { Moderately suppressed } \\
\text { for } 4 \text { hours then normal }\end{array}$ & Normal & Yes & $\begin{array}{l}\text { Yes-Focal } \\
\text { discharge with } \\
\text { sharp features }\end{array}$ & Yes \\
\hline 6 & 1 & Severely suppressed & Normal & No & No & Yes \\
\hline 7 & 2 & $\begin{array}{l}\text { Moderately suppressed } \\
\text { or } 12 \text { hours then normal }\end{array}$ & Normal & $\begin{array}{l}\text { One possible } \\
\text { seizure }\end{array}$ & No & Yes \\
\hline 8 & 2 & $\begin{array}{l}\text { Moderately suppressed } \\
\text { for } 12 \text { hours then normal }\end{array}$ & Normal & $\begin{array}{l}\text { One possible } \\
\text { Seizure }\end{array}$ & No & No \\
\hline 9 & 2 & Normal & Normal & No & No & No \\
\hline 10 & 2 & $\begin{array}{l}\text { Moderately suppressed } \\
\text { for } 30 \text { hours then normal }\end{array}$ & Normal & No & No & Yes \\
\hline 11 & 3 & Normal & Normal & No & No & Yes \\
\hline 12 & 3 & Normal & Normal & No & No & Yes \\
\hline 13 & 3 & Moderately suppressed & Normal & No & No & Yes \\
\hline 14 & 3 & $\begin{array}{l}\text { Moderately Suppressed } \\
\text { for } 12 \text { hours then normal }\end{array}$ & Normal & $\begin{array}{l}\text { One possible } \\
\text { seizure }\end{array}$ & No & Yes \\
\hline 15 & 3 & Normal & Normal & No & No & Yes \\
\hline 16 & 4 & $\begin{array}{l}\text { Normal then sudden change } \\
\text { to continuous seizures at } 42 \\
\text { hours ECMO. Trace became } \\
\text { severely suppressed with } \\
\text { burst suppression }\end{array}$ & $\begin{array}{l}\text { Normal D1 } \\
\text { ECMO } \\
\text { D4 ECMO } \\
\text { Large left } \\
\text { cerebral } \\
\text { haemorrhage }\end{array}$ & No & $\begin{array}{l}\text { Yes - No } \\
\text { seizures }\end{array}$ & No \\
\hline 17 & 4 & Moderately Suppressed & Normal & No & No & No \\
\hline 18 & 4 & Moderately Suppressed & Normal & No & No & No \\
\hline 19 & 4 & $\begin{array}{l}\text { Moderately Suppressed } \\
\text { for } 12 \text { hours then normal }\end{array}$ & Normal & No & No & Yes \\
\hline 20 & 4 & $\begin{array}{l}\text { Moderately suppressed } \\
\text { for } 24 \text { hours then normal }\end{array}$ & Normal & No & No & Yes \\
\hline 21 & 4 & Continuous seizures & $\begin{array}{l}\text { Cerebral } \\
\text { oedema }\end{array}$ & Yes & $\begin{array}{l}\text { Yes - very } \\
\text { abnormal with } \\
\text { bursts of high } \\
\text { amplitude }\end{array}$ & No \\
\hline 22 & 5 & $\begin{array}{l}\text { Moderately Suppressed for } \\
12 \text { hours then normal with } \\
\text { seizure activity }\end{array}$ & Normal & $\begin{array}{l}\text { One Possible } \\
\text { Seizure }\end{array}$ & $\begin{array}{l}\text { Yes - mild } \\
\text { cerebral } \\
\text { dysfunction }\end{array}$ & Yes \\
\hline 23 & 5 & Normal & Normal & No & No & Yes \\
\hline 24 & 5 & $\begin{array}{l}\text { Moderately Suppressed, } \\
\text { continuous seizures } 30- \\
72 \mathrm{hrs,} \text { then moderately } \\
\text { suppressed }\end{array}$ & Normal & Yes & $\begin{array}{l}\text { Yes- periods } \\
\text { of frequent } \\
\text { relative } \\
\text { flattening }\end{array}$ & Yes \\
\hline 25 & 5 & $\begin{array}{l}\text { Moderately Suppressed for } \\
12 \text { hours then normal }\end{array}$ & Normal & $\begin{array}{l}\text { One possible } \\
\text { seizure }\end{array}$ & No & Yes \\
\hline 26 & 5 & $\begin{array}{l}\text { Moderately Suppressed from } \\
6 \text { to } 24 \text { hrs then normal }\end{array}$ & Normal & No & No & Yes \\
\hline
\end{tabular}


Table 3: Summary of aEEG voltage during the last 6 hours of cooling and the first 6 hours after rewarming

\begin{tabular}{|c|c|c|c|c|c|c|}
\hline \multirow[t]{3}{*}{ Patient } & \multicolumn{3}{|c|}{ Cooled } & \multicolumn{3}{|c|}{ Re-warmed } \\
\hline & \multirow{2}{*}{$\begin{array}{l}\text { Rectal Temp } \\
\quad\left({ }^{\circ} \mathrm{C}\right)\end{array}$} & \multicolumn{2}{|c|}{ aEEG } & \multirow{2}{*}{$\begin{array}{l}\text { Rectal Temp } \\
\quad\left({ }^{\circ} \mathrm{C}\right)\end{array}$} & \multicolumn{2}{|c|}{$\mathrm{aEEG}$} \\
\hline & & $\begin{array}{l}\text { Upper } \\
\text { margin } \\
\text { (uvolts) }\end{array}$ & $\begin{array}{l}\text { Lower } \\
\text { margin } \\
\text { (uvolts) }\end{array}$ & & $\begin{array}{l}\text { Upper } \\
\text { margin } \\
\text { (uvolts) }\end{array}$ & $\begin{array}{l}\text { Lower } \\
\text { margin } \\
\text { (uvolts) }\end{array}$ \\
\hline 1 & $36.0(0.11)$ & $19(0)$ & $7(0)$ & $37.1(0.04)$ & $19(0)$ & $7(0)$ \\
\hline 2 & $35.9(0.16)$ & $25(0)$ & $9(0)$ & $37.2(0.18)$ & $25(0)$ & $9(0)$ \\
\hline 3 & $35.9(0.12)$ & $17(0)$ & $7(0)$ & $36.9(0.15)$ & $17(0)$ & $7(0)$ \\
\hline 4 & $34.9(0.04)$ & $20(0)$ & $10(0)$ & $37.1(0.10)$ & $20(0)$ & $10(0)$ \\
\hline 5 & $35.0(0.12)$ & $20(0)$ & $9.2(0.4)$ & $37.0(0.04)$ & $20(0)$ & $9(0)$ \\
\hline 6 & $35.0(0)$ & $25(0)$ & $7(0)$ & $36.9(0.10)$ & $28.3(1.6)$ & $5(0)$ \\
\hline 7 & $35.1(0.18)$ & $22(0)$ & $8.3(0.5)$ & $36.9(0)$ & $22(0)$ & $8(0)$ \\
\hline 8 & $34.9(0.16)$ & $20(0)$ & $7(0)$ & $36.9(0.06)$ & $20(0)$ & $7(0)$ \\
\hline 9 & $33.9(0.1)$ & $20(0)$ & $6(0)$ & $36.9(0.17)$ & $20(0)$ & $6(0)$ \\
\hline 10 & $33.9(0.14)$ & $16(0)$ & $5(0)$ & $36.8(0.15)$ & $16(0)$ & $6(0)$ \\
\hline 11 & $34.1(0.08)$ & $19.8(0.4)$ & $8(0)$ & $36.9(0.05)$ & $19.8(0.4)$ & $8(0)$ \\
\hline 12 & $34.1(0.16)$ & $19(0)$ & $8(0)$ & $36.9(0.12)$ & $19(0)$ & $8(0)$ \\
\hline 13 & $34.1(0.05)$ & $22(0)$ & $9(0)$ & $36.9(0.05)$ & $22(0)$ & $9(0)$ \\
\hline 14 & $34.1(0.14)$ & $15(0)$ & $6.2(1)$ & $37.0(0.08)$ & $15(0)$ & $6.4(0.9)$ \\
\hline 15 & $33.9(0.12)$ & $22(0)$ & $8(0)$ & $36.9(0.05)$ & $22(0)$ & $8(0)$ \\
\hline 16 & $34.1(0.04)$ & $19.6(0.5)$ & $7.8(0.4)$ & $36.9(0.08)$ & $19.6(0.5)$ & $7.8(0.4)$ \\
\hline
\end{tabular}

All values are mean (standard deviation) 
Table 4: Paired Samples Test of aEEG voltage during last 6 hours of cooling and first 6 hours of rewarming

\begin{tabular}{lcccc}
\hline \multicolumn{1}{c}{ aEEG } & $\begin{array}{c}\text { Mean } \\
(\mu \text { volts })\end{array}$ & $\begin{array}{c}\text { No of } \\
\text { samples }\end{array}$ & $\begin{array}{c}\text { Std. } \\
\text { Deviation } \\
(\mu \text { volts })\end{array}$ & $\begin{array}{c}\text { Significance } \\
\text { (2-tailed) }\end{array}$ \\
$\begin{array}{l}\text { Upper margin } \\
\text { (cooled) }\end{array}$ & 19.94 & 8.60 & 0.08 \\
$\begin{array}{l}\text { Upper margin } \\
\text { (rewarmed) }\end{array}$ & 20.07 & 88 & 2.95 & \\
$\begin{array}{l}\text { Lower margin } \\
\text { (cooled) }\end{array}$ & 7.64 & 88 & 1.33 & 0.43 \\
$\begin{array}{l}\text { Lower margin } \\
\text { (rewarmed) }\end{array}$ & 7.60 & 8.29 & \\
\hline
\end{tabular}


Figure 5 Changes in aEEG with Acute Neurological Complications

Trace A

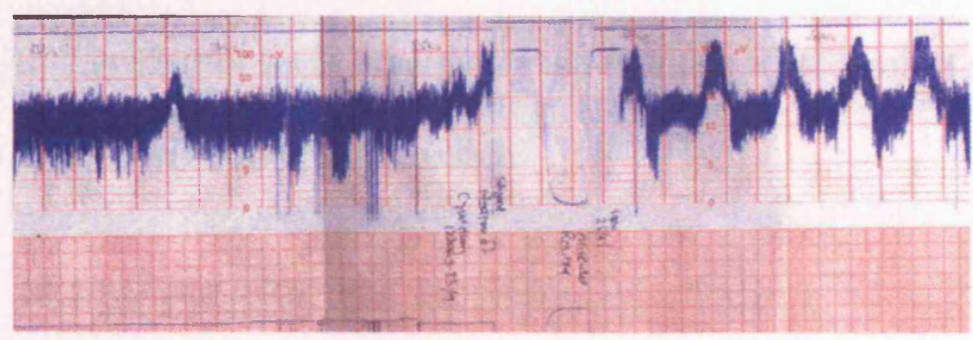

Sudden changes to continuous seizures at 35 hours ECMO associated with cerebral haemorrhage

Trace B

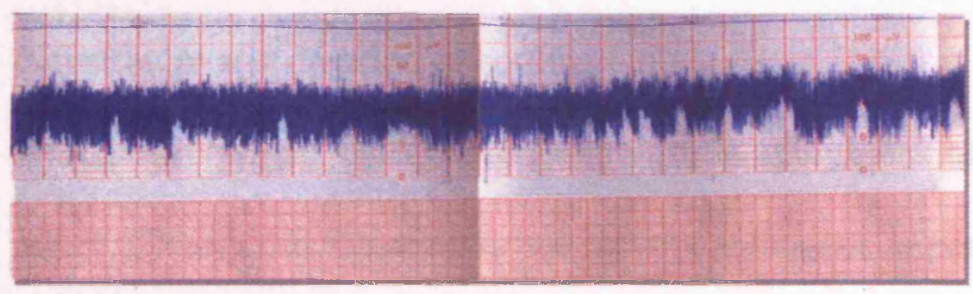

Seizures on aEEG 24 hours prior to clinical seizures 


\section{CHAPTER 10}

\section{SUMMARY AND CONCLUSIONS}

\section{INTRODUCTION}

In this chapter I will summarise the findings of the project and discuss the possible future direction of the research.

\subsection{SUMMARY}

The results of animal investigations have shown that mild hypothermia appears to be a promising means of cerebral neuroprotection following hypoxic-ischaemic injury ${ }^{3-6}$. Translating these experimental results into clinical practice still poses a number of challenges including timing of onset of mild hypothermia, the optimum cooling temperature and duration of cooling.

Neonates receiving ECMO represent a group at high risk of hypoxic ischaemic brain injury. Clearly this situation is not entirely analogous to perinatal asphyxia but it does seem plausible that protecting the brain immediately following cannulation for ECMO may prevent further cerebral injury. Data from adult patients would support the view that mild hypothermia can protect the brain following a variety of insults including cardiac arrest due to ventricular fibrillation. ${ }^{354}$ Regarding timing of applying hypothermia post hypoxic insult, data from Hanrahan et $\mathrm{al}^{86}$ using proton magnetic resonance studies, indicated persistent increase in cerebral lactate concentration for several weeks following birth asphyxia. This supports the view that the "post-insult" neurological damage is not confined to the period immediately after injury. In addition, Taylor et $\mathrm{al}^{247}$ in a study in fourteen day old rat pups, showed improved neuroprotection with hypothermia delayed by six hours 
following hypoxic-ischaemic insult. Hence neuroprotective benefit from cooling may still be possible in neonates referred for ECMO after several hours of hypoxia.

\section{Pilot Study Design}

In the absence of data concerning hypothermia during ECMO a stepwise approach to both temperature reduction and length of cooling time was applied. This allowed careful scrutiny of both the infants and function of the ECMO circuit.

Since this was a pilot study, a power calculation of sample size was not performed. The results of the study will be used to contribute to the power calculation for any subsequent randomised controlled trial.

During the study, I demonstrated that using an extracorporeal circuit to cool neonates was feasible. In addition, I was able to maintain accurate temperature control throughout the study period, which compares favourably with the control achieved using other techniques of cooling. ${ }^{275,344}$

The patients were not randomised to a particular temperature group but instead joined the groups in order. The next reduction in temperature only occurred after the successful completion of the previous stage. As previously stated, the patients in group 4 were scrutinised extremely carefully before continuing with the study. Having proceeded on to cool five infants for forty eight hours all of whom had no major adverse effects during cooling and survived, I believe that I have demonstrated that infants can be safely cooled whilst on ECMO. However, I appreciate that this study was designed to detect large and consistent side effects only and more subtle effects due to temperature may be shown in a randomised controlled trial recruiting larger numbers of patients. 


\section{Adverse Effects of Cooling on the Patient and Extracorporeal Circuit}

In this study I detected no major clinical or circuit problems during cooling or rewarming. In particular, there were no problems of excessive bleeding in patients

cooled to $34^{\circ} \mathrm{C}$. During cooling, the infants became bradycardic but there were no episodes of cardiac arrhythmias. Within the limitations of the study design, mild hypothermia did not appear to impact on the immune response. Finally, cooling to $34^{\circ} \mathrm{C}$ did not change the amplitude on the aEEG.

In terms of the extracorporeal circuit, I did not detect excessive clot formation in circuits nor was there a problem of oxygenator failure during cooling. There was no difference in the heparin requirement between the study groups. However, all patients in group $5\left(34^{\circ} \mathrm{C}\right.$ for 48 hours $)$ showed a consistent rise then fall in heparin requirement during the cooling and rewarming period ( $0-60$ hours). The significance of this observation is not clear but will need further investigation.

\subsection{CONCLUSIONS}

I believe that this pilot study has demonstrated that the use of mild hypothermia in patients receiving ECMO is both feasible and safe for up to 48 hours.

\subsection{FUTURE DIRECTION OF RESEARCH}

I envisage the research to move forward in a number of ways. Firstly, the absence of adverse effects of cooling noted in this study suggests that it is reasonable to proceed to a randomised controlled trial ( $\mathrm{RCT})$ to assess mild hypothermia as a neuroprotective strategy during neonatal ECMO. This trial will require a relatively 
large number of infants to show a neuroprotective effect of mild hypothermia which will require a multicentre trial involving all the UK ECMO centres. In addition, the infants will require standardised neurological follow up at age 2 years to detect differences between the normothermic and cooled infants. Planning for this trial is progressing but there is not yet a definite start date.

Further research is required on the effect of mild hypothermia on coagulation. I would like to further explore the measurement of coagulation parameters at "real temperature". In particular I would revisit the idea of modifying the ACT machine to measure at different temperatures. I would then aim to set up In vitro and In vivo studies to compare the ACT at different temperatures. Similarly, I would also use In vitro studies to compare the standard coagulation tests during cooling and normothermia. Finally, platelet function during cooling could be investigated by means of flow cytometry.

The use of the amplitude-integrated electroencephalogram during ECMO is another area which I would like to investigate further. Although the numbers in my study were small, continuous aEEG recording did seem to be promising as a means of detecting acute neurological changes during ECMO. Clearly, this finding would need to be confirmed in future larger studies. I would propose including aEEG monitoring in the proposed randomised control trial of mild hypothermia during neonatal ECMO. This would give further data on the aEEG changes during ECMO. The predictive power of the aEEG in neurodevelopmental outcome in neonatal ECMO is a complex question to solve due to the number of confounding variables. However, because of the stratification techniques in the study design of the proposed RCT of cooling on ECMO and the standardised neurological follow up, it may be possible to compare the aEEG trace with the neurological outcome in a more meaningful way. 


\subsection{CONTRIBUTION TO THE CURRENT CLINICAL KNOWLEDGE OF MILD HYPOTHERMIA}

Clinical trials of cooling babies who have suffered perinatal asphyxia are currently underway. As discussed in the introduction, two randomised controlled trials of mild hypothermia following neonatal hypoxic ischaemic encephalopathy have been reported in the literature. ${ }^{256,257} \mathrm{~A}$ third randomised controlled trial of whole body cooling in neonatal encephalopathy (the TOBY Trial) is currently underway. However, information from this and previous pilot studies of cooling are of value in planning present and future cooling studies.

I believe that the lack of adverse effects during mild hypothermia demonstrated in my study, particularly with regard to coagulation problems will be of reassurance to those involved in cooling studies. Also, I have shown that a digital monitor can be used to measure QTc interval in neonates however, further validation studies are required to ascertain the normal limits for QTc values. Despite widening of the QTc interval during mild hypothermia, the absence of major cardiovasclular complications including arrhythmias will again be reassuring to other researchers involved in cooling studies of neonates following hypoxic ischaemic injury. Finally I have shown that the aEEG can be used reliably during cooling to $34^{\circ} \mathrm{C}$. 


\section{ACKNOWLEDGEMENTS}

I would like to thank my colleagues, family and friends for their support and invaluable help which has made this thesis possible. I would like to thank the following people in particular who have contributed to this work in different ways.

Firstly I would like to thank my supervisor, Professor David Field for his support and encouragement throughout the project.

The ECMO team at the Glenfield Hospital, in particular Mr Richard Firmin, Hilliary Killer, Gail Faulkner and Giles Peek for their commitment to the project and generous encouragement. The ECMO Co-ordinators, Linda Evans, Guy Cadwalladar and Corrine $\mathrm{M}^{\mathrm{c}}$ Collough deserve special thanks. I am also indebted to the ECMO Specialists and PICU Nursing Staff. Finally, I thank Janice Garner for keeping me sane and helping to locate elusive sets of ECMO charts.

The "Hot Lab" Staff at the Glenfield Hospital for processing the routine blood samples often in the early hours of the morning.

Dr Salesh Kotecha and Professor Andrew Wardlaw for allowing me the use of their laboratories, and their respective staff for their assistance.

Rachel Hodge for her advice and for performing the ELISA assays.

The Department of Electrophysical Investigation at the Glenfield Hospital in particular Mrs Tiena Ablett and Mrs Hilary Rawson for their patience and invaluable assistance in the QTc analysis.

Professor David Edwards and Dr Denis Azzopardi, the Department of Paediatrics, The Hammersmith Hospital, London for their expert advice on mild hypothermia and amplitude integrated electroencephalography. Also I am indebted to both Professor Edwards and Dr Azzopardi for their support throughout the research and advice on analysing the QTc and aEEG data.

The members of the independent monitoring committee, Dr Steve Kerr, PICU Royal Liverpool Children's Hospital, Liverpool and Dr Peter Brocklehurst, National Perinatal Epidemiology Unit Institute of Health Sciences, University of Oxford.

The British Heart Foundation for provision of funding .

The parents of the infants in the study for supporting the research at such a stressful time for themselves and their families.

Last, but not least my family, in particular my sister Bernadette and friends Andrew and Hilary for their support throughout this project and during recent difficult times. 


\section{APPENDIX 1: PAPERS ARISING FROM THE THESIS}

\section{PAPERS}

Horan M, Ichiba S, Firmin R.K, Killer H.M, Edwards A.D, Azzopardi D, Hodge R, Kotecha S, Field D. A Pilot Investigation of Mild Hypothermia in Neonates Receiving Extracorporeal Membrane Oxygenation (ECMO). J Pediatr 2004 Mar; 144(3): 301-8

Horan M, Azzopardi D, Edwards A.D, Firmin R.K, Field D. Lack of Influence of Mild Hypothermia on Amplitude Integrated- Electroencephalograhpy in Neonates Receiving Extracorporeal Membrane Oxygenation. Early Human Dev 2006 Jun 28 (Epub ahead of print)

Horan M, Edwards A.D, Firmin R.K, Ablett T, Rawson H, Field D. The Effect of Temperature on the QTc Interval in the Newborn Infant Receiving Extracorporeal Membrane Oxygenation (ECMO). Early Human Dev 2006 July 10 (Epub ahead of print)

\section{ABSTRACTS}

Horan M, Azzopardi D, Edwards A.D, Firmin R.K, Field D. The Use of Amplitude Integrated Electroencephalography (aEEG) in Neonates Receiving Extracorporeal Membrane Oxygenation (ECMO). Pediatr Res 2004; 55(2): 583A

Horan M, A.D Edwards, Azzopardi D, Firmin RK, Field D. The Relationship between the QT and the QTc Interval and Temperature During Whole Body Mild Hypothermia in Neonates. Arch Dis Child 2003; 88(Suppl 1) A48 - A49.

Horan M, Ichiba S, Firmin R.K, Killer H.M, Edwards A.D, Azzopardi D, Hodge R, Kotecha S, Field D. A Pilot Investigation of Mild Hypothermia in Neonates Receiving Extracorporeal Membrane Oxygenation (ECMO). Pediatr Res 2003 April; 53(4): 23A 


\section{STATISTICAL METHODS}

Data were inspected for normality, and appropriate parametric or non-parametric tests were used. Patient data (gestational age, age at ECMO, birth weight, Apgar score at one and five minutes, $\mathrm{pH}$ before cannulation, length of time on ECMO and oxygenation index) were compared by the Kruskal-Wallis test (SPSS Version 10.1). I used the Kruskal-Wallis for comparison between groups since the data was not normally distributed and the sample size was small. The Kruskal - Wallis is a nonparametric test which is used to compare three or more samples and is particularly often used for small sample sizes. The Kruskal - Wallis is used to test the null hypothesis that all populations have identical functions against the alternative hypothesis that at least two of the samples differ only with respect to location (median), if at all. However, with very small numbers and five different groups the power of the Krusal-Wallis test to detect a real difference between the groups is limited. Therefore for cytokines, Thrombin -antithrombin III, Antithrombin III and plasmin- $\alpha 2$ plasminogen the data were expressed as median and range values. Significance was assumed when the $\mathrm{p}$ value was less than 0.005 . In chapter 5, the relation between temperature and QTc interval over the first 48 hours of the study period and during rewarming, was analysed using logistical regression (Stata V.7 Statacorps, Tx USA). The potential covariates (see chapter 5) were assessed by univariate logistic or linear regression analysis. The digital QTc interval for the first 24 hours for each group was expressed as mean $\left(95^{\text {th }}\right.$ centile) values. The mean value was used in this case because of the large amount as well as the normal distribution of the QTc data analysed for each patient. 


\section{BIBLIOGRAPHY}

1. UK Collaborative ECMO Trial group. UK collaborative randomised trial of neonatal extracorporeal membrane oxygenation. The Lancet. 1996;348:75-82

2. Bennett CC, Johnson A, Field DJ, Elbourne D, for the UK Collaborative ECMO Trial group. UK collaborative randomised trial of neonatal extracorporeal membrane oxygenation: follow-up to age 4 years. The Lancet. 2001;357:1094-1096

3. Thoresen M, Penrice J, Lorek A, Cady EB, Wylezinska M, Kirkbride V et al. . Mild hypothermia following severe transient hypoxia-ischaemia ameliorates delayed cerebral energy failure in the newborn piglet. Pediatr Res. 1995;37:667-670.

4. Gunn AJ, Gunn TR, De Haan HH et al. Dramatic neuronal rescue with prolonged selective head cooling after ischemia in fetal lambs. J Clin Invest 1997;99:248-256.

5. Sirimanne ES, Blumberg RM, Bossano D, Gunning M, Edwards AD, Gluckman PD et al. The effect of prolonged modification of cerebral temperature on outcome following hypoxic-ischaemic brain injury in the infant rat. Pediatr Res 1996;39:591597

6. Colbourne F, Corbett D. Delayed postischemic hypothermia: a six month survival study using behavioral and histological assessments of neuroprotection. $J$ Neurosci. 1995;15(11):7250-7260

7. Freysz M, Timour Q, Mazze RI, Bertrix L, Cohen S, Samii K et al. Potentiation by mild hypothermia of ventricular conduction disturbances and reentrant arrhythmias induced by bupivacaine in dogs. Anesthesiol. 1989;70(5):799-804

8. Okada M. The cardiac rhythm in accidental hypothermia. J Electrocardiol. 1984; $17(2): 123-8$ 
9. Illia R, Ovsyshcher I, Rudnik L et al. Atypical ventricular tachycardia and alternating Osborn waves induced by spontaneous mild hypothermia. Pediatr Cardiol 1998;9:63-5.

10. Sheffield CW, Sessler DI, Hunt TK. Mild hypothermia during isoflurane anesthesia decreases resistance to $E$. coli dermal infection in guinea pigs. Acta Anaesthesiol Scand 1994;38:201-5.

11. VanOss CJ, Asbolam DR, Moore LL. Effect of temperature on chemotaxis, phagocytic engulfment, digestion, and oxygen consumption of human polymorphonuclear leukocytes. J Reticuloendothel Soc 1980;27:561-5.

12. Johnson TD, Chen Y, Reed LR. Functional equivalence of hypothermia to specific clotting factor deficiences. J Trauma. 1994;37:413-7

13. Rohrer MJ, Natale AM. Effect of hypothermia on the coagulation cascade. Crit Care Med. 1992;20:1402-5

14. Valeri CR, Cassidy G, Khuri S, Feingold HM, Ragno G, Altschule MD. Hypothermia -induced reversible platelet dysfunction. Ann Surg. 1987;205:175-81 15. Oung CM, Li MS, Shum-Tim D, Chiu RC, Hinchey EJ. In vivo study of bleeding time and arterial hemorrhage in hypothermic versus normothermic animals. $J$ Trauma. $1993 ; 35(2): 251-4$

16. Richardson BW. An enquiry into the possibility of restoring the life of warm blooded animals in certain cases where respiration, the circulation and the ordinary manifestations of organic motion are exhausted or have ceased. Proc Roy Soc London 1865: $358-371$

17. $\mathrm{M}^{\mathrm{c}}$ Lean J. The discovery of heparin. Circulation 1959; 19: 75-78. 
18. Lewis FJ, Taufic M. Closure of atrial septal defects with the aid of hypothermia: experimental accomplishments and the report of one successful case. Surgery 1953; $33: 52-9$

19. Gibbon JH Jr. Artificial maintenance of circulation during experimental occlusion of the pulmonary artery. Arch Surg 1937;34:1105

20. Gibbon JH Jr. The maintenance of life during experimental occlusion of the pulmonary artery followed by survival. Surg Gynecol Obstet 1939; 69: 602 21. Gibbon JH Jr. Application of a mechanical heart and lung apparatus to cardiac surgery. Minn Med 1954;37:171-85

22. Warden HE, Cohen M, Read RC, Lillehei CW. Controlled cross circulation of open intracardiac surgery. $J$ Thorac Surg 1954;28: 331.

23. Lillehei CW, Varco RL, Cohen $\mathrm{M}$ et al. The first open heart repairs of ventricular septal defect, atrioventricular communis, and tetralogy of Fallot using extracorporeal circulation by cross circulation: a 30 year follow up. Ann Thorac Surg 1986;41:4-21

24. Lillehei CW, DeWall RA, Read RC, Warden HE, Varco RL. Direct vision intracardiac surgery in man using a simple, disposable artificial oxygenator. Dis Chest $1956 ; 29: 1-8$

25. Jones RE, Donald DE, Swan HJC, Harshbarger HG, Kirklin JW, Wood EH. Apparatus of the Gibbon type for mechanical bypass of the heart and lungs. Proceedings of the staff meetings of the Mayo Clinic 1955 March 23;30:105 26. Kirklin JW, Dushane JW, Patrick RT, et al. Intracardiac surgery with the aid of a mechanical pump oxygenator system (Gibbon Type): report of eight cases. Proceedings of the staff meetings of the Mayo Clinic 1955 May 18;30:201 27. Kammermeyer K. Silicone rubber as a selective barrier. Ind Eng Chem 1957;49: 1685 
28. Kolobow T, Bowman RL. Construction and evaluation of an alveolar membrane artificial lung. Trans ASAIO 1963;9:238

29. Kloff WJ, Effler DB. Disposable membrane oxygenator (heart lung machine) and its use in experimental and clinical surgery while the heart is arrested with potassium citrate according to the Melrose technique. Trans ASAIO 1956;2:13-21

30. Bramson ML, Osborn JJ, Main FB et al. A new disposable membrane oxygenator with integral heat exchanger. J Thorac Cardiovascular Surg 1965;50:391

31. Bartlett RH, Isherwood J, Moss RA, Olszweski WL, Polet H, Drinker PA. A toroidal flow membrane oxygenator: four day partial bypass in dogs. Surg Forum $1969 ; 20: 152-3$

32. Bartlett RH, Burns NE, Fong SW, Gazzaniga AB, Achauer BM, Fraille J. Prolonged partial venoarterial bypass: physiologic, biochemical, and hematological responses. Surg Forum 1972;23:178-80

33. Fong SW, Burns NE, Williams G, Woldanski C, Gazzaniga AB, Bartlett RH. Changes in coagulation and platelet function during prolonged extracorporeal circulation (ECC) in sheep and man. Trans ASAIO 1974;20;239-46

34. Hill JD, O’Brien TG, Murray JJ, Dontigny L, Bramson ML, Osborn JJ et al. Extracorporeal membrane oxygenation for acute post-traumatic respiratory failure (shock-lung syndrome). Use of the Bramson membrane lung. $N$ Eng $J$ Med 1972; 286: 62

35. Schulte HD. Membrane oxygenators in prolonged assisted extracorporeal membrane oxygenation. Dtsch Med Wochenschr 1973; 98:508 36. Geelhoed GW, Adkins PC, Corso PJ, Joseph WL. Clinical effects of membrane lung support for acute respiratory failure. Ann Thorac Surg 1975; 20:177-86 
37. Zapol WM, Snider MT, Hill JD, Fallat RJ, Bartlett RH, Edmunds LH et al. Extracorporeal membrane oxygenation in severe respiratory failure. JAMA 1979; 242:2193-6

38. Bartlett RH. Esperanza (ASAIO Presidential Address).

Trans ASAIO 1985;30: 723-5

39. Hill JD, de Leval MR, Fallat RJ et al. Acute respiratory insufficiency: treatment with prolonged extracorporeal oxygenation. J Thorac Cardiovasc Surg 1972;64:51162

40. Soeter JR, Mamiya RT, Sprague AY, M $^{\mathrm{C}}$ Namara JJ. Prolonged extracorporeal oxygenation for cardiorespiratory failure after tetrology correction. $J$ Thorac Cardiovasc Surg 1973; 66: 214-8

41. Bartlett RH, Andrews AF, Toomasian JM, Haidue NJ, Gazzaniga AB. Extracorporeal membrane oxygenation (ECMO) in neonatal respiratory failure: 45 cases. Surgery 1982; 92: 425-53

42. Bartlett RH, Roloff DW, Cornell RG, Andrews AF, Dillon PW, Zwischenberger JB. Extracorporeal circulation in neonatal respiratory failure: a prospective randomised study. Pediatr 1985; 76: 479-487

43. O’Rourke PP, Krone R, Vacanti J, Ware JH, Lillehei CW, Parad RB et al. Extracorporeal membrane oxygenation and conventional medical therapy in neonates with persistent pulmonary hypertension of the newborn: a prospective randomized study. Pediatr 1989; 84: 957-963

44. UK Collaborative ECMO Group. The Collaborative UK ECMO Trial: Follow -up to 1 year of age. Pediatr 1998; 101(4): 1-10

45. Gattinoni L, Pesenti A, Mascheroni D, Marcolin R, Fumagalli R, Rossi F et al. Low- frequency positive-pressure ventilation with extracorporeal $\mathrm{CO}_{2}$ removal in 
severe acute respiratory failure. $J A M A 1986 ; 256: 881-86$

46. Knoch M. Treatment of severe ARDS with extracorporeal $\mathrm{CO}_{2}$ removal. In Gille JP ed Neonatal and Adult Respiratory Failure. Paris; Elsevier, 1989

47. Bindslev L. Extracorporeal circulation using surface heparinised equipment. In Gille JP, ed Neonatal and Adult Respiratory Failure. Paris; Elsevier, 1989

48. Conrad SA, Rycus PT. The registry of the Extracorporeal Life Support Organization. In ECMO Extracorporeal cardiopulmonary support in critical care $2^{\text {nd }}$ Edition pages 295-310

49. Palmer RMJ, Rees DD, Ashton DS, Moncada S. L-arginine is the physiological precursor for the formation of nitric oxide in endothelium-dependent relaxation. Biochem Biophys Res Commun 1988; 153: 1251-1256

50. Ignarro LJ, Adams JB, Horwitz PM, Wood KS. Activation of soluble guanylate cyclase by NO-hemoproteins involves NO-heme exchange. J Biol Chem 1986; 261 : 4997-5002

51. Frostell C, Fratacci MD, Wain JC, Jones R, Zapol WM. Inhaled nitric oxide- A selective pulmonary vasodilator reversing hypoxic pulmonary vasoconstriction. Circulation 1991; 83: 2038- 2047

52. Fratacci MD, Frostell CG, Chen TY, Wain JC, Robinson DR, Zapol WM. Inhaled nitric oxide- A selective pulmonary vasodilator of heparin-protamine vasoconstriction in sheep. Anesthesiol 1991; 75: 990-999

53. Roberts JD, Chen TY, Kawai N, Wain J, Dupuy P, Shimouchi A, Bloch K, Polaner D, Zapol WM. Inhaled nitric oxide reverses pulmonary vasoconstriction in the hypoxic and acidotic newborn lamb. Circ Res 1993; 72: 246-254

54. Roberts JD, Polaner DM, Lang P, Zapol WM. Inhaled nitric oxide in persistent pulmonary hypertension of the newborn. Lancet 1992; 340: 818-819 
55. Kinsella JP, Neish SR, Shaffer E, Abman SH. Low-dose inhalation nitric oxide in persistent pulmonary hypertension of the newborn. Lancet 1992; 340: 819-820

56. The NINOS Study Group: Inhaled nitric oxide for near -term infants with respiratory failure. $N$ Eng $J$ Med 1997; 336: 602-605

57. Finer NN, Barrington KJ. Nitric oxide in respiratory failure in infants born at or near term. Cochrane Database Syst Rev 2000; (2): CD000399

58. The Neonatal Inhaled Nitric Oxide Study Group (NINOS). Inhaled nitric oxide and hypoxic respiratory failure in infants with congenital diaphragmatic hernia.

Pediatr 1997; 99: 838-45

59. The Neonatal Inhaled Nitric Oxide Study Group (NINOS). Inhaled nitric oxide in term and near term-infants: neurodevelopmental follow-up of the neonatal inhaled nitric oxide study group (NINOS). J Pediatr 2000; 136: 611-7

60. Weimann J, Ullrich R, Hromi J, Fujino Y, Clark MW, Bloch KD, Zapol WM.

Sildenafil is a pulmonary vasodilator in awake lambs with acute pulmonary hypertension. Anesthesiol 2000; 92(6): 1702-12

61. Shekerdemian LS, Ravn HB, Penny DJ. Intravenous sildenafil lowers pulmonary vascular resistance in a model of neonatal pulmonary hypertension. Am J Resp Crit Care Med 2002; 165(8): 1098-102

62. Michelakis E, Tymachak W, Lien D, Webster L, Hashimoto K, Archer S. Oral sildenafil is an effective pulmonary vasodilator in patients with pulmonary arterial hypertension: comparison with inhaled nitric oxide. Circulation 2002; 105(20): 2398 $-403$

63. Finer NN. Neonatal Selection Criteria for ECMO. ECMO Extracorporeal Cardiopulmonary Support in Critical Care $2^{\text {nd }}$ Edition; 357-362

64. Firmin RK, Killer HM. Extracorporeal membrane oxygenation. Perfusion 1999; 
65. Bulas DI, Taylor GA, Odonnell RM, Short BL, Fitz CR, Vezina G. Intracranial abnormalities in infants treated with extra corporeal membrane oxygenation: Update on sonographic and CT findings. Am J Neuroradiol 1996; 17(2): 287 - 94

66. Oritz RM, Cilley RE, Bartlett RH. Extracorporeal membrane oxygenation in pediatric respiratory failure. Pediatr Clin N Amer 1987; 34:39-46

67. Krummel BV, Greenfield LJ, Kirkpatrick BV, Meuller DG, Kerkering KW, Ormazabal M, Meyers EC, Napolitano A, Barnes RW, Salzberg AM et al. Alveolararterial oxygen gradient versus the Neonatal Pulmonary Insufficiency Index for prediction of mortality in ECMO candidates. J Pediatr Surg 1984; 19: 380 - 384 68. Beck R, Anderson KD, Pearson GD, Cronin J, Miller MK, Short BL. Criteria for extracorporeal membrane oxygenation in a population of infants with persistent pulmonary hypertension of the newborn. J Pediatr Surg 1986; 21: 297 - 302

69. Bohn D, Tamura M, Perrin D, Barker G, Rabinovitch M. Ventilatory predictors of pulmonary hypoplasia in congenital diaphragmatic hernia, confirmed by morphological assessment. J Pediatr 1987; 111: 423-431

70. Conrad S, Rycus P. Extracorporeal life support 1997. ASAIO J 1998; 44: 848-52 71. Stewart D, Mendoza J, Winston S, Cook L. Extracorporeal life support (ECLS) in infants with total anomalous pulmonary venous drainage (TAPVD): a review of the ELSO registry. CNMC ECMO Symposium 1992: 81

72. Doski J, Butler J, Louder D, Dickey L, Cheu H. Outcome of infants requiring cardiopulmonary resuscitation before extracorporeal membrane oxygenation. $J$ Pediatr Surg 1997; 32: 1318-21

73. Glass P, Wagner A, Papero P, Rajasingham S, Civitello L, Kjaer M, Coffman C, 
Getson P, Short B. Neurodevelopmental status at age five years of neonates treated with extracorporeal membrane oxygenation. $J$ Pediatr 1995; 127: 447-57

74. Adolph V, Ekelund C, Smith C, Starrett A, Falterman K, Arensman R.

Developmental outcome of neonates treated with ECMO. J Pediatr Surg 1990; 25:

$43-6$

75. Hofkosh D, Thompson A, Nozza R, Kemp S, Bowen A, Feldman H. Ten years of ECMO: neurodevelopmental outcome. Pediatr 1991; 87: 549-55

76. Schumacher R, Palmer T, Roloff D, LaClaire P, Bartlett R. Follow-up of infants treated with ECMO for newborn respiratory failure. Pediatr 1991; 87: 451-7

77. Wildin S, Landry S, Zwischenberger J. Prospective, controlled study of developmental outcome in survivors of ECMO: the first 24 months. Pediatr 1994; 93: $404-14$

78. Boggs K, Visser V. ECMO treatment: one year outcomes for North Carolina infants. NC Med J 1991; 52: 423-6

79. Cheung P, Haluschak M, Finer N, Robertson C. Sensorineural hearing loss in survivors of neonatal extracorporeal membrane oxygenation. Early Human Dev 1996; 44: $225-33$

80. Bernbaum J, Schwartz IP, Gerdes M, D’Agostino, Coburn CE, Polin RA. Survivors of extracorporeal membrane oxygenation at 1 year of age: The relationship of primary diagnosis with health and neurodevelopmental sequelae. Pediatr 1995; 96(5): $907-913$

81. Kornbauser MS, Baumgart S, Shobhana A, Stanley CW, Culhane J, Cullen JA, Wiswell TE, Graziana LJ, Spitzer AR. Adverse neurodevelopmental outcome after extracorporeal membrane oxygenation among neonates with bronchopulmonary dysplasia. J Pediatr 1998; 132(2): 307-311 
82. Vaucher YE, Dudell GG, Bejar R, Gist K. Predictors of early childhood outcome in candidates for extracorporeal membrane oxygenation. $J$ Pediatr 1996; 128: 109-

\section{7}

83. Kirino T. Delayed neuronal death in the gerbil hippocampus following ischemia. Brain Res 1997; 239: 57-69

84. Hope PL, Costello AM, Cady EB, Delpy DT, Tofts PS, Chu A, Hamilton PA, Reynolds EOR, Wilkie DR. Cerebral energy metabolism studied with phosphorus NMR spectroscopy in normal and birth- asphyxiated infants. Lancet 1984; 2: 366-370 85. Hanrahan D, Sargentoni J, Azzopardi D, Manji K, Cowan F, Rutherford MA, Cox IJ, Bell DJ, Bryant D, Edwards AD. Cerebral metabolism within 18 hours of asphyxia: a proton magnetic resonance spectroscopy study. Pediatr Res 1996; 39(4): 584-590

86. Hanrahan D, Cox J, Edwards AD, Cowan FM, Sargentoni J, Bell JD, Bryant DJ, Rutherford MA, Azzopardi D. Persistent increases in cerebral lactate concentration after birth asphyxia. Pediatr Res 1998; 44(3): 304-311

87. Martin E, Buchli R, Ritter S, Schmid R, Largo RH, Boltshauser E, Fanconi S, Duc G, Rumpel H. Diagnostic and prognostic value of cerebral 31P magnetic resonance spectroscopy in neonates with perinatal asphyxia. Pediatr Res 1996; 40: 749-758 88. Roth SC, Edwards AD, Cady EB, Delpy DT, Wyatt JS, Azzopardi D, Baudin J, Townsend J, Stewart AL, Reynolds EOR. Relation between cerebral oxidative metabolism following birth asphyxia, and neurodevelopmental outcome and brain growth at one year. Dev Med Child Neurol 1992; 34: 285-295

89. Roth SC, Baudin J, Cady EB, Johal K, Townsend JP, Wyatt JS, Reynolds EOR, 
Stewart AL. Relation of deranged neonatal cerebral oxidative metabolism with neurodevelopmental outcome and head circumference at 4 years. Dev Med Child Neurol 1997; 39: 718-725

90. Lorek A, Takei Y, Cady EB, Wyatt JS, Penrice J, Edwards AD, Peebles D, Wylezinska M, Owen-Reece H, Kirkbride V, Cooper CE, Aldridge RF, Roth SC, Brown G, Delpy DT, Reynolds EOR. Delayed (“secondary”) cerebral energy failure after acute hypoxia-ischemia in the newborn piglet: continuous 48-hour studies by phosphorus magnetic resonance spectroscopy. Pediatr Res 1994; 36(6): 699-706 91. Palmer C, Brucklacher RM, Christensen MA, Vannuci RC. Carbohydrate and energy metabolism during evolution of hypoxic-ischemic brain damage in the immature rat. J Cereb Blood Flow Metab 1990; 10: 227-235 92. Yager JY, Brucklacher RM, Vannucci RC. Cerebral energy metabolism during hypoxia-ischaemia and early recovery in immature rats. Am J Physiol Heart Circ Physiol 1992; 262: H672-H677

93. Williams CE, Gunn AJ, Mallard C, Gluckman PD. Outcome after ischemia in the developing sheep brain: An electroencephalographic and histological study. Annals Neurol 1992; 31(1): 14-20

94. Tan WK, Williams CE, During MJ, Mallard CE, Gunning MI, Gunn AJ, Gluckman PD. Accumulation of cytotoxins during the development of seizures and edema after hypoxia-ischaemic injury in late gestation fetal sheep. Pediatr Res 1996; 39(5): 791-797.

95. Taylor DL, Edwards AD, Mehmet H. Oxidative metabolism, apoptosis and perinatal brain injury. Brain Pathol. 1999; 9:93 - 117

96. Thornton JS, Ordidge RJ, Penrice J, Cady EB, Amess PN, Punwani S, Clemence 
M, Wyatt JS. Anisotropic water diffusion in white and gray matter of the neonatal piglet brain before and after transient hypoxia-ischaemia. Magnetic Resonance Imaging 1997; 15(4): 433-440.

97. Rutherford MA, Pennock JM, Schweiso J, Cowan F, Dubowitz L. Hypoxic-ischaemic enecephalopathy: early and late magnetic resonance imaging findings in relation to outcome. Arch Dis Child 1996; 75: F145-51

98. Penrice J, Cady E, Lorek A, Wylezinska M, Amess P, Aldridge R, Stewart A, Wyatt JS, Reynolds EOR. Proton magnetic resonance spectroscopy of the brain in normal preterm and term infants, and early changes after perinatal hypoxia-ischaemia. Pediatr Res 1996; 40: 6-14

99. Yue X, Mehmet H, Squier MV, Hope PL, Azzopardi D, Edwards AD. Apoptosis and necrosis in the brains of infants dying after birth asphyxia. Pediatr Res $1995 ; 37$ : $387 \mathrm{~A}$

100. Mehmet H, Yue X, Squier MV et al. Increased apoptosis in the cingulate sulcus of newborn piglets following transient hypoxia-ischaemia is related to the degree of high energy phosphate depletion during the insult. Neurosci Lett 1994; 181 : $121-5$

101. Ferrer I, Tortosa A, Macaya A, et al. Evidence of nuclear DNA fragmentation following hypoxia-ischaemia in the infant rat brain, and transient forebrain ischaemia in the adult gerbil. Brain Pathol 1994; 4: 115-22

102. Beilharz E, Williams CE, Dragunow M, Sirimanne E, Gluckman PD. Mechanisms of cell death following hypoxia-ischaemic injury in the immature rat: evidence of apoptosis during selective neuronal loss. Mol Brain Res 1995; 29: 1-14 103. Linnik MD, Zobrist RH, Hatfield MD. Evidence supporting a role for 
programmed cell death in focal cerebral ischaemia in rats. Stroke 1993; 24: 2002$387 \mathrm{~A}$

104. Wyllie AH, Duvall E. Cell injury and death. In: McGee JO'D, Isacsson PG, Wright NA, eds Oxford textbook of pathology. Oxford: Oxford University Press, 1992: $141-93$

105.Trump BF, Berezesky IK, Cowley RA. The cellular and subcellular characteristics of acute and chronic injury with emphasis on the role of calcium. In: Cowley RA, Trump BF, (Eds), 1982; Pathophysiology of shock, anoxia, and ischemia. Williams and Wilkins, Baltimore, MD, pp. 6-46

106. Wyllie AH. Cell death: a new classification separating apoptosis from necrosis. In: Bowen ID, Lockshin RA. (Eds), Cell death in biology and pathology 1981; Chapman and Hall, London, pp. 9-34)

107. Walker NI, Harmon BV, Gobe GC, Kerr JFR, 1988. Patterns of cell death. Methods Achiev. Exp. Pathol 13; 1988: 18-54.

108. Banasiak KJ, Xia Y, Haddad GG. Mechanism underlying hypoxia-induced neuronal apoptosis. Progress in Neurobiol 2000; 62: 215-249

109. Wyllie AH. Glucocorticoid -induced thymic apoptosis is associated with endogenous nuclease activation. Nature 1980; 284: 555-556

110. Gavrieli Y, Sherman Y, Ben-Sasson SA. Identification of programmed cell death in situ via specific labeling of nuclear DNA fragmentation . J Cell Biol 1992; 119:

$493-501$

111. Vermes I, Haanen C, Steffens-Nakken H, Reutelingsperger C. A novel assay for apoptosis. Flow cytometric detection of phosphatidylserine expression on early apoptotic cells using fluorescein labelled Annexin V. J Immunol Methods 1995; 184: $39-51$ 
112. Raff MC. Social controls on cell survival and cell death.

Nature 1992; 356:397-400

113. Oppenheim RW. Cell death during development of the nervous system. Ann Rev Neurosci 1991; 14: 453-501

114. Thomas CB. Apoptosis in the pathogenesis and treatment of disease. Science 1995; 267: 1456-62 114.

115.Greenberg ME, Greene LA, Ziff EB. Nerve growth factor and epidermal growth factor induce rapid transient changes in proto-oncogene transcription in PC12 cells. $J$ Biol Chem 1985; 260: 14101-14110

116. Greenberg ME, Ziff EB, Green LA. Stimulation of the neuronal acetylcholine receptors induces rapid gene transcription. Science; $234: 80-83$

117. Morgan JI, Curran T. Role of ion flux in the control of c-fos expression. Nature; 322: 552-555

118. Hughes $\mathrm{P}$, Dragunow M. Induction of immediate-early genes and the control of neurotransmitter -regulated gene expression within the nervous system. Pharmac Rev 1995; 47: 133-178

119. Levine AJ. p53, the cellular gatekeeper for growth and division.

Cell 1997; 88: 328-331

120. Giaccia AJ, Kastan MB. The complexity of p53 modulation: emerging patterns from divergent signals. Genes Dev 1998; 12: 2973-2983

121. Crook T, Parker GA, Rozycka M, Crossland S, Allday MJ. A transforming p53 mutant, which binds DNA, transactivates and induces apoptosis reveals a nuclear:cytoplasmic shutting defect. Oncogene 1998; 16:1492-1441 122. Chao DT, Korsmeyer SJ. Bcl-2 family: regulators of cell death. Ann Rev Immunol 1998; 16: 395-419 
123.Tsujimoto Y, Croce CM. Analysis of the structure, transcripts, and protein products of the bcl-2, the gene involved in human follicular lymphoma. Proc Natl. Acad. Sci USA 1986; 83: 5214-5218

124. Boise LH, Thompson CB. Bcl-xl can inhibit apoptosis in cells that have undergone Fas-induced protease activation. Proc Natl Acad Sci USA 1997; 94: 37593764

125. Schendel SL, Xie Z, Montal MO, Matsuyama S, Montal M, Reed JC. Channel formation by antiapoptotic protein bcl-2. Proc Natl Acad USA 1997; 94: 5113-5118 126. Lam M, Bhat MB, Nunez G, Ma J, Distelhorst CW. Regulation of bcl-xl channel activity by calcium. $J$ Biol Chem 1998; 273: 17307-17310

127. Kluck RM, Bossy-Wetzel E, Green DR, Newmeyer DD, Schulze-Osthoff K. The Fas/APO1 receptor and its deadly ligand. Trends Cell Biol 1994; 4: 421-7 128. Hannun YA, Obleid LM. Ceramide: an intracellular signal apoptosis. Trends Biochem Sci 1995; 20: 73-7

129. Tang DG, Li L, Zhu Z, Joshi B. Apoptosis in the absence of cytochrome c accumulation in the cytosol. Biochem Res Commun 1998; 242(2): 380-4 130. Chauhan D, Pandey P, Otaga A, Teoh G, Krett N, Halgren R, Rosen S, Kufe D, Kharbanda S, Anderson K. Cytochrome c-dependent and -independent induction of apoptosis in multiple myeloma cells. J Biol Chem 1997; 272(48): 29995-29997 131. Montal M. Mitochondria, glutamate neurotoxicity and the death cascade. Biochem Biophys Acta 1998; 1366: 113-126

132. Wang HG, Pathan N, Ethell IM, Krajewksi S, Yamaguchi Y, Shibasaki F, McKeon F, Bobo T, Franke TF, Reed JC. $\mathrm{Ca}^{2+}$ - induced apoptosis through calcineurin dephosphorylation of BAD. Science 1999a; 284: 339-343 133. Neame SJ, Rubin LL, Philpott KL. Blocking cytochrome c activity within intact 
neurons inhibits apoptosis. J Cell Biol 1998; 142: 1583-1593

134. Fonnum F. Glutamate: A neurotransmitter in mammalian brain.

J Neurochem 1984; 42: 1-11

135. Rothman SM, Olney JW. Glutamate and the pathophysiology of hypoxic-ischaemic brain damage. Ann Neurol 1986; 19: 105-11

136. Kass IS, Lipton P. Mechanisms involved in irreversible anoxic damage to the in vitro rat hippocampal slice. J Physiol 1982; 332: 459-472

137. McBean GJ, Roberts PJ. Chronic infusion of 1-glutamate causes neurotoxicity in rat striatum. Brain Res 1984; 290: 372-375

138. Steiner HX, McBean GJ, Kohler C et al. Ibotenate -induced neuronal degeneration in immature rat brain. Brain Res 1984; 307: 117-124 139. Szatkowaski M, Attwell D. Triggering and execution of neuronal death in brain ischaemia: two phases of glutamate release by different mechanisms. Trends Neurosci 1994; 17: 359-365

140. Albers G, Goldberg MP, Choi DW. N-methyl-D-aspartate antagonists: Ready for clinical trial in brain ischaemia? Ann Neurol 1989; 25: 398-403

141. McDonald JW, Silverstein FS, Johnston MV. MK -801 protects the neonatal brain from hypoxic-ischaemic damage. Eur J Pharmacol 1987; 140: 359-361

142. Ford LM, Sanberg PR, Norman AB. MK-801 prevents hippocampal neurodegeneration in neonatal hypoxic-ischemic rats. Arch Neurol 1989; 46: 10901096

143. Hattori H, Morin AM, Schwartz PH, et al. Post- hypoxic treatment with MK-801 reduces hypoxic-ischemic damage in the neonatal rat. Neurology 1989; 39: 713-718 144. Tan WK, Williams CE, Gunn AJ, Mallard CE, Gluckman PD. Suppression of 
postischemic epileptiform activity with MK-801 improves neural outcome in fetal sheep. Ann Neurol 1992; 32: 677-682

145. McAuley MA. Rodent models of focal ischaemia. Cerebrovasc Brain Metab Rev $1995 ; 7: 153-180$

146. Small DL, Buchan AM. NMDA antagonists: their role in neuroprotection. Int Rev Neurobiology 1997; 40: 137-171

147. Nakajima W, Ishida A, Takada G. Magnesium attenuates a striatal dopamine increase induced by anoxia in the neonatal rat brain: An in vivo microdialysis study. Pediatr Res 1997; 41(6): 809-814

148. McDonald JW, Silverstein FS, Johnston MV. Magnesium reduces N-methyl -Daspartate (NMDA)-mediated brain injury in perinatal rats. Neurosci Lett 1990; 109: $234-238$

149. Marret S, Gressens P, Gadisseux JF, Evrard P. Prevention by magnesium of excitotoxic neuronal death in the developing brain: an animal model for clinical intervention studies. Dev Med Child Neurol 1995; 37: 473-484 150. Penrice J, Amess P, Punwani S, Wylezinska M, Tyszczuk L, D'Souza P et al. Magnesium sulphate after transient hypoxia-ischaemia fails to prevent delayed cerebral energy failure in the newborn piglet. Pediatr Res 1997; 41: 443-449 151. Greenwood K, Cox P, Mehmet H, Penrice J, Amess PN, Cady EB, Wyatt JS, Edwards AD. Magnesium sulfate treatment after transient hypoxia-ischemia in the newborn piglet does not protect against cerebral damage. Ped Res 2000; 48(3): 346350

152. de Haan HH, Gunn AJ, Williams CE, Heymann MA, Gluckman PD. Magnesium sulfate therapy during asphyxia in near term fetal lambs does not compromise the fetus but does reduce cerebral injury. Am J Obstet Gynecol 1997; 176: 18-27 
153. Nelson KB, Grether JK. Can magnesium sulfate reduce the risk of cerebral palsy in very low birthweight infants. Pediatr 1995; 95: 263-269

154. Mittendorf R, Covert R, Boman J, Khoshnood B, Lee KS, Seigler M. Is tocolytic magnesium sulphate associated with increased total paediatric mortality? Lancet $1997 ; 350: 1517-1519$

155. Benichou J, Zupan V, Fernandez H, Marpeau L, Marret S. Tocolytic magnesium sulphate and paediatric mortality. Lancet 1997; 351:290-29

156. Levene MI, Evans DJ, Mason S, Brown J. An international network for evaluating neuroprotective therapy after severe birth asphyxia. Sem Perinatol 1999; 23: $226-233$

157. Nowicki JP, Duval D, Poignet H, Scatten B. Nitric oxide mediates neuronal death after focal cerebral ischemia in the mouse. Eur J Pharmacol 1991; 204: 339 340

158. Dawson VL, Dawson TM, London ED, Bredt DS, Snyder SH. Nitric oxide mediates glutamate neurotoxicity in primary cortical cultures. Proc Natl Acad Sci USA 1991; 88: $6368-6371$

159. Dawson VL, Dawson TM, Bartley DA, Uhl GR, Snyder SH. Mechanisms of nitric oxide-mediated neurotoxicity in primary brain cultures. $J$ Neurosci $1993 ; 13$ : $2651-2661$

160. Dawson VL, Dawson TM. Nitric oxide neurotoxicity. J Chem Neuroanat 1996; 10: $179-190$

161. Thoresen M, Satas S, Puka-Sandvall, Whitelaw A, Hallstrom A, Loberg EM, Ungerstedt U, Steen PA, Hagberg H. Post-hypoxic hypothermia reduces cerebrocortical release of NO and excitotoxins. Neuroreport 1997; 8: 3359 - 3362 162. Brown GC, Cooper CE. Nanomolar concentrations of nitric oxide reversibly 
inhibit synaptomosal respiration by competing with oxygen at cytochrome oxidase. FEBS Lett 1994; 356: 259 - 298

163. Ankarcrona M, Dypbukt JM, Bonofoco E, Zhivotovsky B, Orrenius S, Lipton SA, Nicotera P. Glutamate - induced neuronal death: a succession of necrosis or apoptosis depending on mitochondrial function. Neuron 1995; 15: 961 - 973 164. Khan S, Kayahara M, Joashi U, Mazarakis ND, Sarraf C, Edwards AD et al. Differential induction of apoptosis in Swiss $3 \mathrm{~T} 3$ cells by nitric oxide and nitrosonium cation. J Cell Sci 1997; 110: 2315 - 2322

165. Beckman JS, Koppenol WH. Nitric oxide, superoxide, and peroxynitrite: the good, the bad, and ugly. Am J Physiol 1996; 27: C1424 - C1437.

166. Samdani AF, Dawson TM, Dawson VL. Nitric oxide synthase in models of focal ischemia. Stroke 1997; 28: 1283 - 1288

167. Marks KA, Mallard CE, Roberts I, Williams CE, Gluckman PD, Edwards AD. Nitric oxide synthase inhibition attenuates delayed vasodilation and increases injury after cerebral ischemia in fetal sheep. Pediatr Res;40(2):185 - 191

168. Ferriero DM, Holtzman DM, Black SM, Sheldon RA. Neonatal mice lacking neuronal nitric oxide synthases are less vulnerable to hypoxic-ischaemic injury. Neurobiol Dis 1996; 3: 64 - 71

169. Peeters-Scholte C, Koster J, Veldhuis W, van-den Tweel E, Zhu C, Kops N, Blomgren K, Bar D, van Buul-Offers S, Hagberg H, Nicolay K, van Bel F, Groenendaal F. Neuroprotection by selective nitric oxide synthase inhibition at 24 hours after perinatal hypoxia-ischemia. Stroke 2002; 33: 2304-2310.

170. Blumberg RM, Taylor DL. Xu Y, Kripamoy A, McKenzie J, Cady EB, Weiner 
$\mathrm{CP}$, Mehmet $\mathrm{H}$, Edwards AD. Increased nitric oxide synthesis is not involved in delayed cerebral energy failure following focal hypoxic-ischemic injury to the developing brain. Pediatr Res 1999; 46(2): 224 - 231

171. Hagberg H, Andersson P, Lacarewicz J, et al. Extracellular adenosine inosine, hypoxanthine and xanthine in relation to tissue nucleotides and purines in rat striatum during transient ischemia. $J$ Neurochem 1987; 49: 227 - 231

172. Legido A. Perinatal hypoxic-ischemic encephalopathy: current advances in diagnosis and treatment. Int Pediatr 1994;9: 114 - 136

173. Tan S, Parks DA. Preserving brain function during neonatal asphyxia.

Clin Perinatol 1999; 11: $223-228$

174. Palmer C, Vanucci RC, Towfighi J. Reduction of perinatal hypoxic-ischemic brain damage with allopurinol. Ped Res 1990; 27: 332 - 336

175. Williams GD, Palmer C, Heitjan DF et al. Allopurinol preserves cerebral energy metabolism during perinatal hypoxic-ischemia: a 31P NMR study in anaesthetised immature rats. Neurosci Lett 1992; 144: 103-106

176. Palmer C, Roberts RL. Reduction of perinatal brain damage with oxypurinol treatment after hypoxic-ischemic injury. Pediatr Res 1991; 29: 362A

177. Russell GA, Cooke RW. Randomised controlled trial of allopurinol prophylaxis in very preterm infants. Arch Dis Child Fetal Neonatal Ed 1995; 73: F27 - F31 178. Van Bel F, Shadid M, Moison RM, et al. Effect of allopurinol postasphyxial free radical formation, cerebral hemodynamics, and electrical brain activity. Pediatr 1998; $101: 185-193$

179. Imaizumi S, Wollworth V, Fishman RA et al. Superoxide dismutase activities and their role in focal cerebral ischemia. J Cereb Blood Flow Metab 1989; 9: S217 180. Liu TH, Beckman JS, Freeman BA, et al. Polyethylene glycol-conjugated 
superoxide dismutase and catalase reduce ischemic brain injury. Am J Physiol 1989;256: H589 -H593

181. Takashima S, Kuruta H, Mito T et al. Immunohistochemistry of superoxide dismutase-1 in developing human brain. Brain Dev 1990; 12: 211 - 213

182. Sullivan JL: Iron metabolism and oxygen radical injury in premature infants. In Lauffer RB(ed): Iron and Human Disease. Boca Raton, FL, CRC Press, 1992, pp $447-455$

183. Palmer C, Vanucci RC. Potential new therapies for perinatal cerebral hypoxia-ischaemia. Clin Perinatol 1993; 20: 411 - 432

184. Van der Worp HB, Bar PR, Kappelle LJ. Dietary vitamin E levels affect outcome of permanent focal cerebral ischemia in rats. Stroke 1998; 29: 1002 - 1005 185. Stohrer M, Eichinger A, Schlachter M, Stangassinger M. Protective effect of vitamin $\mathrm{E}$ in a rat model of cerebral ischemia in rats. $Z$ Naturforsch 1998; 53: 273 278

186. Nakajima M, Kashiwagi K, Hayashi Y et al. Alpha - tocopherol supports the survival and neurite extension of neurones cultured from various regions of fetal rat brain. Neurosci Lett 1991; 133: 49 - 52

187. Tagami M, Yamagata K, Ikeda K et al. Vitamin E prevents apoptosis in cortical neurones during hypoxia. Lab Invest 1998; 78: 1415 - 1429

188. Hall ED, Pazara KE, Braughler JM: Effects of tirilazad mesylate on postischemic brain lipid peroxidation and recovery of extra cellular calcium in gerbils. Stroke 1991;22: 361-366

189. Boisvert DP. Effectiveness of postischemic 21-amino steroid U-74006F in preventing reperfusion brain edema. J Cereb Blood Flow Metab 1991; 11: S135 190. Bagenholm R, Andine P, Hagberg H et al. Effects of 21-aminosteroid U74006F 
on brain damage and edema following perinatal hypoxia-ischemia in the rat. $J$ Cereb Blood Flow Metab 1991; 11: S134

191. Amar AP, Levy ML. Pathogenesis and pharmacological strategies for mitigating secondary damage in acute spinal cord surgery. Neurosurg 1999; 44(5): 1027 - 39 192. Evan GI. Old cells never die, they just apoptose. Trends Cell Biol 1994;

4: $191-192$

193. Linnik MD, Zahos P, Geschwind MD, Federoff HJ. Expression of bcl-2 from a defective herpes simplex virus-1 vector limits neuronal death in focal cerebral ischemia. Stroke 1995; 26: $1670-1675$

194. Lawrence MS, Ho DY, Sun GH, Steinberg GK, Sapolsky RM. Overexpression of bcl-2 with herpes simplex virus vectors protects CNS neurons against neurological insults in vitro and in vivo. $J$ Neurosci 1996; 16: $486-496$

195. Martinou J, Dubois-Dauphin M, Staple J et al. Overexpression of bcl-2 in transgenic mice protects neurons from naturally occurring death and experimental ischemia. Neuron 1994; 13: $1017-1030$

196. Lawrence MS, McLaughlin JR, Sun GH et al. Herpes simplex viral vectors expressing bcl-2 are neuroprotective when delivered after stroke.J Cerebr Blood Flow Metab 1997; 17: 740 - 744

197. Mishra OP, Delivoria-papadopoulos M. Anti-oxidant enzymes in fetal guinea pig brain during development and the effect of maternal hypoxia. Dev Brain Res 1988; 42: $173-179$

198. Macgregor DG, Higgins MJ, Jones PA et al. Ascorbate attenuates the systemic kainate-induced neurotoxicity in the rat hippocampus. Brain Res 1996; 727: 133 - 144 199. Tan S, Zhou F, Wang ZW et al. Administration of antioxidants to the mother 
after onset of fetal bradycardia ameliorates fetal brain injury in rabbits. Pediatr Res 1997; 41: 182A

200. Hisanaga K, Sagar SM, Sharp FR. Ascorbate neurotoxicity in cortical cell culture. Ann Neurol 1992; 31: 562 - 565

201. Bass WT, Malati N, Castle MC, et al. Evidence for the safety of ascorbic acid administration to the premature infant. Am J Perinatol 1998; 15: $133-140$

202. Jeggo PA. DNA repair: PARP-another guardian angel? Curr Biol 1998;

8:R49 - R51

203. Takahashi K, Greenberg JH, Jackson P, Maclin K, Zhang J. Neuroprotective effects of inhibiting Poly(ADP-ribose) Synthetase on focal cerebral ischaemia in rats. J Cereb Blood Flow Metab 1997; 17: 1137 - 1142

204. Eliasson MJL, Sampei K, Mandir AS, Hurn PD, Traystman RJ, Bao J et al. Poly(ADP) ribose polymerase gene disruption renders mice resistant to cerebral ischaemia. Nature Med 1997; 3: 1089 - 1095

205. Holtzman DM, Sheldon RA, Jaffe W, Cheng Y, Ferriero DM. Nerve growth factor protects the neonatal brain against hypoxic -ischemic injury. Ann Neurol 1996; 39: $114-122)$

206. Cheng Y, Gidday JM, Yan Q, Shah AR, Holtzman DM. Marked age-dependent neuroprotection by brain derived neurotrophic factor against neonatal hypoxicischemic brain injury. Ann Neurol 1997; 41: 521 - 529

207. Guan J, Williams C, Gunning M, Mallard C, Gluckman P. The effects of IGF-1 treatment after hypoxic-ischemic brain injury in adult rats. J Cereb Blood Flow Metab $1993 ; 13: 609-616$

208. Gluckman PD, Klempt N, Guan J, Mallard C, Sirimanne E, Dragunow M et al. 
A role for IGF-1 in the rescue of CNS neurons following hypoxic-ischemic injury. Biochem Biophys Res Commun 1992; 182: 593 - 599

209. Johnston BM, Mallard EC, Williams CE, Gluckman PD. Insulin-like growth factor -1 is a potent neuronal rescue agent hypoxic-ischaemic injury. $J$ Clin Invest 1996; 97: $300-308$

210. Guan J, Bennet L, George S et al. Selective neuroprotective effects with insulin-like growth factor-1 in phenotypic striatal neurons following ischemic brain injury in fetal sheep. Neuroscience 2000; 95: $831-839$

211. Legido A. Pathophysiology of perinatal hypoxic-ischemic encephalopathy. Acta Neuropediatr 1994; 1: 97-110

212. Painter MJ. Animal models of perinatal asphyxia; contributions, clinical relevance. Sem Pediatr Neurol 1995; 2: 37 - 56

213. Du Plessis AJ, Johnston MV. Hypoxic-ischemic brain injury in the newborn. Cellular mechanisms and potential strategies for neuroprotection. Clin Perinatol $1997 ; 24: 627-654$

214. Silverstein FS, Buchanan K, Hudson C et al. Flunarazine limits hypoxicischemia induced morphologic injury in immature rat brain. Stroke 1986; 17: 477 482

215. Gunn AJ, Gluckman PD. Flunarazine, a calcium channel antagonist, is not neuroprotective when given after hypoxia-ischemia in the infant rat. Dev Pharmacol Ther 1991; 17: 205 - 209

216. Zapater P, Moreno J, Horga JF. Neuroprotection by the novel calcium antagonist PCA50938, nimodipine and flunarizine in gerbil global brain ischemia. Brain Res $1997 ; 772: 57-62$

217. Franke CL, Palm R, Dalby M, et al. Flunarizine in stroke treatment (FIST): 
a double blind, placebo-controlled trial in Scandinavia and the Netherlands. Acta Neurol Scand 1996; 93: 56-60

218. Roda JM, Carceller F, Diez-tejedor et al. Reduction of infarct size by intra-arterial nimodipine administered at reperfusion in a rat model of partially reversible brain focal ischemia. Stroke 195; 26: 1888 - 1892

219. Satoh S, Ikegaki I, Suzuki Y, et al. Neuroprotective properties of a protein kinase inhibitor against ischaemia - induced neuronal damage in rats and gerbils. $\mathrm{Br} J$ Pharmacol 1996; 118: 1592 - 1596

220. Raju TN, John E, Shankararao R et al. Effect of nimodipine on systemic, renal, and cerebral haemodynamics after a mild hypoxic-ischemic insult in newborn piglets. Pharmacol Res 1996; 33: 5 -12

221. Gelmers HJ, Gorer K, de Weerdt CJ, Wiezer HJ. A controlled trial of nimodipine in acute ischemic stroke. New Engl J Med 1988; 318: 203 - 7

222. Sze KH, Sim TC, Wong E, et al. Effect of nimodipine on memory after cerebral infarction. Acta Neurol Scand 1998; 97: 386 - 392

223. Levene MI, Gibson NA, Fenton AC, Papathoma E, Barnett D. The use of a calcium-channel blocker, nicardipin for severely asphyxiated newborn infants. Dev Med Child Neurol 1990; 32: 567 - 574

224. Sarco DP, Becker J, Palmer C, Sheldon RA, Ferriero DM. The neuroprotective effect of deferoxamine in the hypoxic-ischemic immature mouse brain. Neurosci Lett $2000 ; 282: 113-116$

225. Palmer C, Roberts RL, Bero C. Deferoxamine post-treatment reduces ischemic brain injury in neonatal rats. Stroke 1994; 25(5): 1039-1045

226. Vanucci RC, Perlman JM. Interventions for perinatal hypoxic-ischemic encephalopathy. Pediatr 1997; 100: $1004-1014$ 
227. Tan WKM, Williams CE, Gunn AJ et al. Pretreatment with monosialoganglioside $\mathrm{GM}_{1}$ protects the brain of fetal sheep against hypoxic-ischemic injury without causing systemic compromise. Pediatr Res 1993; 34: 18-22 228. Tan WKM, Williams CE, Mallard CE et al. Monosialoganaglioside $\mathrm{GM}_{1}$ treatment after a hypoxic -ischemic episode reduces the vulnerability of the fetal sheep brain to subsequent injuries. Am J Obstet Gynecol 1994;170: 663 - 670 229. Lenzi GL, Grigoletti F, Gent M et al. Early treatment of stroke with monosialogangliosides $\mathrm{GM}_{1}$. Efficacy and safety results of the early stroke trial. Stroke 1994; 25: 1552 - 1558

230. Phillis JW, O’Regan $\mathrm{MH} . \mathrm{GM}_{1}$ ganglioside inhibits ischemic release of amino acid neurotransmitters from rat cortex. Neuroreport 1995; 23: 2010 - 2012 231. Thoresen M, Bagenholm R, Loberg EM, Apricena F, Kjellmer I. Posthypoxic cooling of neonatal rats provides protection against brain injury. Arch Child Dis. 1996; 74: F3-F9

232. Yager J, Towfighi J, Vannucci RC. Influence of mild hypothermia on hypoxic -ischemic brain damage in the immature rat. Pediatr Res 1993; 34(4): 525 529

233. Bona E, Johansson BB, Hagberg H. Sensorimotor function and neuropathology five to six weeks after hypoxia-ischaemia in seven-day-old rats. Pediatr Res 1997;42: $678-683$

234. Williams GD, Dardzinski BJ, Buckalew AR, Smith MB. Modest hypothermia preserves cerebral energy metabolism during hypoxic-ischaemia and correlates with brain damage: A 31P nuclear magnetic resonance study in unanaesthetised neonatal rats. Pediatr Res 1997; 42: 700-8

235. Yager JY, Brucklacher RM, Mujsce DJ, Vannucci RC. Cerebral oxidative 
metabolism during hypothermia and circulatory arrest in newborn dogs. Pediatr Res $1992 ; 32: 547-52$

236. Edwards AD, Wyatt JS, Thoresen M. Treatment of hypoxic-ischaemic brain damage by moderate hypothermia. Arch Dis Child Fetal Neonatal Ed 1998; 78: F85F91

237. Busto R, Globus MY, Dietrich WD, Martinez E, Vaides I, Ginsberg MD. Effect of mild hypothermia on ischaemia induced release of neurotransmitters and free fatty acids in the rat brain. Stroke $1989 ; 20: 904-10$

238. Huang R, Shuaib A, Hertz L. Glutamate uptake and glutamate content in primary cultures of mouse astrocytes during anoxia, substrate deprivation and simulated ischemia under normothermic and hypothermic conditions. Brain Res 1993; 618: 34651 239. Tan WK, Williams CE, During MJ et al. Accumulation of cytotoxins under normothermic and hypothermic conditions. Brain Res 1993; 618: 346-51 240. Frank SM, Higgins MS, Breslow MJ et al. The catecholamine, cortisol, and haemodynamic responses to mild perioperative hypothermia. A randomised clinical trial. Anesthesiology 1995; 82: 83-93

241. Thoresen M, Bagenholm R, Loberg E, Apricena F. The stress of being restrained reduces brain damage after a hypoxic-ischaemic insult in the seven day old rat. Neuroreport 1997; 7: 481-3

242. Edwards AD, Yue X, Squier MV et al. Specific inhibition of apoptosis after cerebral hypoxic-ischaemia by moderate post- insult hypothermia. Biochem Biophys Res Commun 1995; 217: 1193-9

243. Kozma M, Ravirajan G, Edwards AD, Mehmet H. Moderate hypothermia 
reduces apoptosis in cultured neuronal cells deprived of nerve growth factor. Pediatr Res $1995 ; 38: 441$

244. Guan J, Williams CE, Skinner SJ, Mallard EC, Gluckman PD. The effects of insulin-like growth factor (IGF)-1, (IGF)-2, and des-IGF-1 on neuronal loss after hypoxic-ischemic brain injury in adult rats: evidence for a role for IGF binding proteins. Endocrinology 1996; 137: 893-8

245. Klempt ND, Sirimanne E, Gunn AJ et al. Hypoxic-ischemia induces transforming growth factor $1 \mathrm{mRNA}$ in the infant rat brain. Brain Res Mol Brain Res $1992 ; 13: 93-101$

246. Dietrich WD, Busto R, Globus MY, Ginsberg MD. Brain damage and temperature:cellular and molecular mechanisms. Adv Neurol 1996; 71: 177-94 247. Taylor DL, Mehmet H, Cady EB, Edwards AD. Improved neuroprotection with hypothermia delayed by 6 hours following cerebral hypoxia-ischemia in the 14-dayold rat pup. Pediatr Res 2002; 51(1): 13-19

248. Laptook AR, Shalak L, Corbett RJT. Differences in brain temperature and cerebral blood flow during selective head versus whole body cooling. Pediatr 2001; 108(5): $1103-1110$

249. Van Leeuwen GMJ, Hand JW, Lagendijk JJW, Azzopardi DV, Edwards AD. Numerical modeling of temperature distributions within the neonatal brain. Pediatr Res 2000; 48(3): 351-356

250. Thoresen M, Simmonds M, Satas S, Tooley J, Silver IA. Effective selective head cooling during posthypoxic hypothermia in newborn piglets. Pediatr Res 2001; 49(4):594-599

251. Corbett RJ, Laptook AR, Tollefsbol G, Kim B. Validation of noninvasive 
method to measure brain temperature in vivo using $1 \mathrm{H}$ NMR spectroscopy. $J$ Neurochem 1995; 64: 1224-30

252. Cady EB, D’Souza PC, Penrice J, Lorek A. The estimation of local brain temperature by in vivo $1 \mathrm{H}$ magnetic resonance spectroscopy. Magnetic Resonance Med 1995; 33: 862-7

253. Gunn AJ, Gunn TR. Effect of radiation heat on head temperature gradient in near term infants. Arch Dis Child 1996; 74: F200-3

254. Simbruner G, Nanz S, Fleischbacker E, Derganc M. Brain temperature discriminates between neonates with damaged, hypoperfused, and normal brains. Ann J Perinatol 1994; 11: 137-43

255. Roberts I, Azzopardi D, Hand J, Edwards AD. Discrimination of deep from surface temperature with emitted microwave radiation in a brain-specific phantom. Early Human Development (in press).

256. Gluckman PD, Wyatt JS, Azzopardi D, Ballard R, Edwards AD, Ferriero DM et al. Selective head cooling with mild systemic hypothermia to improve neurodevelopmental outcome after neonatal encephalopathy: Multicentre randomised trial. Lancet 2005;365: 663-670

257.Shankaran S, Laptook AR, Ehrenkranz RA, Tyson JE, McDonald SA et al. Whole body hypothermia for neonates with hypoxic-ischaemic encephalopathy. $\mathrm{N}$ Eng J Med 2005; 353: 1574-84

258. Patt A, $\mathrm{M}^{\mathrm{C}}$ Croskey BL, Moore EE. Hypothermia -induced coagulopathies in trauma. Surg Clin North Am 1988; 68: 775-85

259. Reed RL 2d, Johnston TD, Hudson JD, Fischer RP. The disparity between hypothermic coagulopathy and clotting studies. J Trauma 1992; 33: 465-70 260. Michelson AD, MacGregor H, Barnard MR, Kestin AS, Rohrer MJ, Valeri RC. 
Reversible inhibition of human platelet activation by hypothermia in vivo and in vitro. Semin Thromb Hemost 1994;71(5): 633 - 40

261. Cohen IJ. Room temperature ADP-induced first-stage hyperaggregation of human platelets: the cause of rewarming deaths by thrombocytopenia in neonatal cold injury. Pediatr Hematol Oncol 1991;8:61-7

262. Schmied H, Kurz A, Sessler DI, Kozek S, Reiter A. Mild intraoperative hypothermia increases blood loss and allogeneic blood requirements during total hip arthroplasty. Lancet 1996; 347: 289-92

263. Ferrara A, MacArthur JD, Wright HK, Modulin IM, M $^{\mathrm{C}}$ Millen MA. Hypothermia and acidosis worsen coagulopathy in the patient requiring massive transfusion. Am J Surg 1990;160: 515-8

264. Kettner SC, Kozek SA, Groetzner JP, Gonano C, Schellonogowski A, Kucera M, Zimpfer M. Effects of hypothermia on thromboelastography in patients undergoing cardiopulmonary bypass. Br J Anaesth 1998;80:313-7

265. Reuler JB. Hypothermia: pathophysiology, clinical settings, and management. Ann Intern Med 1978; 89: 519-27

266. Lilly RB. Inadvertent hypothermia: a real problem. ASA Refresher Course Lectures 1987;15: 93-107

267. Chen RYZ, Chien S. Haemodynamic functions and blood viscosity in surface hypothermia. Am J Phyisol 1978; 235: H136-43

268. Frank SM, Fleisher LA, Breslow MJ, Higgins MS, Olson KF, Kelly S, Beattie C. Perioperative maintenance of normothermia reduces the incidence of morbid cardiac events: A randomised clinical trial. JAMA 1997; 277:1127-34

269. Frank SM, El-Gamal N, Raja SN, Wu PK. Alpha-adrenoceptor mechanisms of thermoregulation during cold challenge in humans. Clin Sci 1996; 91:627 - 31 
270. Green PS, Cameron DE, Mohlala ML, Dinatale JM, Gardner TJ. Systolic and diastolic left ventricular dysfunction due to mild hypothermia. Circulation 1989; 80: III 44-8

271. Dudgeon DL, Randall PA, Hill RB, McAfee JG. Mild hypothermia: its effect on cardiac output and regional perfusion in the neonatal piglet. J Pediatr Surg 1980; 15: 805- 810

272. Riishede L, Nielsen - Kudks F. Myocardial effects of adrenaline, isoprenaline and dobutamine at hypothermic conditions. Pharmacol Toxicol 1990; 66: 354-60 273. Ree M J. Electrocardiographic changes in accidental hypothermia. Br Heart $J$ 1964; 26: 566

274. Thompson R, Rich J, Chmelik F, Nelson W. Evolutionary changes in the electrocardiogram of severe progressive hypothermia. J Electrocardiol 1977; 10(1): $67-70$

275. Thoresen M, Whitelaw A. Cardiovascular changes during mild therapeutic hypothermia and rewarming in infants with hypoxic-ischemic encephalopathy. Pediatr. 2000; 106(1): 92-99

276. Mackenzie M.A, Aengevaeren W.R.M, Van der Werf T, Hermus Ad R.M.M, Kloppenborg P.W.C. Effects of steady hypothermia and normothermia and the electrocardiogram in human poikilothermia. Arct Med Res 1991;50:Suppl.6:67-70287. 277. Gunn TR, Wilson NJ, Aftimos S, Gunn AJ. Brain hypothermia and QT interval. Pediatr 1999;103(5):1079

278. Boelhouwer RU, Bruning HA, Ong GL. Correlations of serum potassium fluctuations with body temperature after major surgery. Crit Care Med 1987; 15: 310 $-2$

279.Sprung J, Cheng EY, Gamulin S, Kampine JP, Bosnjak ZJ. The effect of acute 
hypothermia and serum potassium concentration on potassium cardiotoxicity in anesthetized rats. Acta Anaesthesiol Scand 1992; 36: 825-30

280. Koht A, Cane R, Cerullo LJ. Serum potassium levels during prolonged hypothermia. Intensive Care Med 1983; 9: 275-7

281. Farkas LG, Bannantyne RM, James JS, Umamaheswaran B.

Effect of two different climates on severely burned rats infected with pseudomonas aeruginosa. Eur Surg Res 1974; 6: 295-300

282. Sarian K, Nickerson DA. Enhancement of murine in vitro antibody formation by hyperthermia. Cell Immunol 1982; 74: 306-12

283. Sessler DI. Complications and Treatment of Mild Hypothermia. Anesthesiol $2001 ; 95: 531-43$

284. Sessler DI. Perianesthetic thermoregulation and heat balance in humans.

FASEB J 1993; 7: 638-644

285. Michenfelder JD, Theye RA. Hypothermia: effect on canine brain and whole body metabolism. Anesthesiology 1968; 29: 1107- 1112

286. Steen PA, Soule EH, Michenfelder JD. Detrimental effect of prolonged hypothermia in cats and monkeys with and without regional cerebral ischemia. Stroke $1979 ; 10: 522-9$

287. Steen PA, Milde JH, Michenfelder JD. The detrimental effects of prolonged hypothermia and rewarming in the dog. Anesthesiol 1980; 52: $224-30$

288. Levene M, Wright I, Griffiths G. Is informed consent in neonatal randomised trials ritual ? Lancet 1996; 347: 475

289. Modi N. Informed consent difficult for paediatric intensive care.

British Medical Journal 1993; 307: 1495

290. Firmin RK, Waggoner J, Pearson GA. Extracorporeal membrane oxygenation for 
neonates and older children. Mechanical Circulatory Support, (Lewis T, Graham TR, eds) 1995 Edward Arnold: 78 -95

291. (ELSO) ELSO, Experience from the extracorporeal life support organisation (ELSO) registry. 2000

292. Cork RC, Vaughan RW, Humphrey LS. Precision and accuracy of intraoperative temperature monitoring. Anesth Analg 1983; 62: 211-4

293. Eichna LW, Berger AR, Rader B, Buckaroo WH. Comparison of intracardiac and intravascular temperatures with rectal temperatures in man. J Clin Invest 1951;

30: $353-9$

294. Yetman RJ, Coody DK, West MS, Montgomery D, Brown M. Comparison of temperature measurement by an aural infrared thermometer with measurements by traditional rectal and axillary techniques. J Pediatr 1993; 122: 769-73

295.Giuliano KK, Scott SS, Ellot S, Giuliano AJ. Temperature measurement in critically ill orally intubated adults: A comparison of pulmonary artery core, tympanic, and oral methods. Crit Care Med 1999; 27(10) 2188-2193 296.Craig JV, Lancaster GA, Taylor S, Williamson PR, Smyth RL. Infrared ear thermometry compared with rectal thermometry in children: a systematic review. Lancet 2002; 360: 603-09

297.Loveys AA, Dutko-Fioravanti I, Eberly SW, Powell KR. Comparison of ear to rectal temperature measurements in infants and toddlers. Clin Pediatr 1999; August: 463-466

298.Cattaneo CC, Frank SM, Hesel TW, El-Rahmany HK, Kim LJ, Tran KM.

The accuracy and precision of body temperature monitoring methods during regional and general anesthesia. Anesth Analg 2000; 90: 938-45

299.Erickson RS, Kirklin SK.Comparison of ear-based, bladder, oral, and axillary 
methods for core temperature measurement. Crit Care Med 1993; 21(10): 1528-1534 300. Mravinac CM, Dracup K, Clochesy JM. Urinary bladder and rectal temperature monitoring during clinical hypothermia. Nurs Res 1989; 38(2): 73-76

301. Zerbe RL, Robertson GL. Osmotic and nonosmotic regulation of thirst and vasopressin secretion. In M.H. Maxwell, CR Kleeman, \& RG Narins (Eds), Clinical disorders of fluid and electrolyte metabolism (pp 61-78). New York: McGraw-Hill Book Co., Inc

302. Robinson JL, Seal RF, Spady DW, Joffres MR.Comparisons of oesophageal, rectal, axillary, bladder, tympanic, and pulmonary artery temperatures in children. $J$ Pediatr 1998; 133: 553-56

303.Romano MJ, Fortenberry JD, Autrey E, Harris S, Heyroth T, Parmeter P, Stein F. Infrared tympanic thermometry in the pediatric intensive care unit. Crit Care Med 1993; 21(8): $1181-5$

304. Clinical effectiveness committee for management of infants of acute fever of uncertain source in infants 60 days of age or less. Cincinatti Children's Hospital Medical Center, 1998. http://www.guidelines.gov

305. Morley CJ, Thornton AJ, Cole TJ, Hewson PH, Fowler MA. Baby check: a scoring system to grade the severity of acute systemic illness in babies under 6 months old. Arch Dis Child 1991; 66: 100- 106 306. NG D. Infrared ear thermometers versus rectal thermometers. Lancet 2002 Dec 7; 360: (9348):1881 307. Okado M, Nishimura F, Yoshino H, Kimura M, Ogino T. The $\mathrm{J}$ wave in accidental hypothermia. $J$ Electrocardiol 1983; 16(1): 23-28 308. Rankin AC, Rae AP. Cardiac arrhythmias during rewarming of patients with accidental hypothermia. Br Med $J$ 1984;289:874-877 
309. Nordrehug JE. Sustained ventricular fibrillation in deep accidental hypothermia. Br Med J 1982;284:867-8

310. Towne WD, Geiss MD, Yanes HO, Rahimtoola SH. Intractable ventricular fibrillation associated with profound accidental hypothermia - successful treatment with partial cardiopulmonary bypass. $N$ Eng $J$ Med 1972;287:1135-6

311. Schwartz PJ, Montemerlo M, Facchini M, Salice P, Rosti D, Poggio G et al. The QT interval throughout the first 6 months of life: A prospective study. Circulation $1982 ; 66(3): 496-501$

312. Rijnbeek PR, Witsenburg M, Schrama E, Hess J, Kors JA. New normal limits for the paediatric electrocardiogram. Eur Heart $J$ 2001; 22: 702 - 711

313. Davignon A, Rautaharju P, Boisselle E, Soumis F, Megelas M, Choguette A. Normal ECG standards for infants and children. Pediatr Cardiol 1979/80;1:123-31 314. Lande G, Funck - Brentano, Ghadanfar M, Escande D. Steady-state versus nonsteady-state QT-RR relationships in 24-hour Holter recordings.

$P A C E$ 2000;23:293-

315. Homs E, Marti V, Guindo J, Laguna P, Vinolas X, Caminal P et al. Automatic measurement of corrected QT interval in Holter recordings:Comparison of its dynamic behavior in patients after myocardial infarction with or without lifethreatening arrhythmias. Am Heart $J$ 1997;134:181-7

316. Crawford MH, Bernstein SJ, Deedwania PC, DiMarco JP, Ferrick KJ, Garson A et al. ACC/AHA Guidelines for ambulatory electrocardiography:executive summary and recommendations. A report of the American College of Cardiology/American Heart Association task force on practice guidelines (committee to revise the guidelines for ambulatory electrocardiography). Circulation 1999;100:886-893 
317. Stowe DF, Fujita S, An J, Paulsen RA, Varadarajan SG, Smart SC. Modulation of myocardial function and $\left[\mathrm{Ca}^{2+}\right]$ sensitivity by moderate hypothermia in guinea pig isolated hearts. Am J Physiol 1999;277(heart circ. Physiol.46):H2321-2332 318. Kiyosue T, Muramatsu H, Spindler AJ, Noble D. Ionic mechanisms of action potential prolongation at low temperature in guinea-pig ventricular myocytes. $J$ Physiol 1993;468:85-106

319. Yan G, Antzelevitch C. Cellular basis for the electrocardiographic J wave. Circulation 1996;93:372-379

320. Villalobos T.J, Adelson E, Barila TG. Hematologic changes in hypothermic dogs. Proc Soc Exp Biol Med 1955; 89:192-196

321. Chan A.K.C, Leaker M, Burrows F.A, Williams W.G, Gruenwald C.E, Whyte L Adams M, Brooker L.A, Adams H, Mitchell L, Andrew M. Coagulation and fibrinolytic profile of paediatric patients undergoing cardiopulmonary bypass. Thromb Haemost 1997;77:270-277

322. Woodman R.C, Harker LA. Bleeding complications associated with cardiopulmonary bypass. Blood 1990;76:1680-1697

323. Bick R.L. Hemostasis defects associated with cardiac surgery, prosthetic devices, and other extracorporeal circuits. Seminars in Thromb Hemost $1985 ; 11: 249-280$

324. Shimokawa M, Kitaguchi K, Kawaguchi M, Sakamoto T, Kakimoto M, Furuya $\mathrm{H}$. The influence of induced hypothermia for hemostatic function on temperature- adjusted measurements in rabbits. Anesth Analg. 2003;96:12091213

325. Shimokawa M, Kitaguchi K, Furuya H. Influence of mild hypothermia on temperature adjusted clot analysis in neurosurgical patients. Jpn J Haematology 
$2003 ; 21: 18-21$

326. Douning LK, Ramsay MA, Swygert TH et al. Temperature corrected Thromboelastography in hypothermia patients. Anesth Analg 1995; 81: $608-11$ 327. Hattersley PG. Heparin anticoagulation. In Keopke JA(editor): Laboratory Hematology. Churchill Livingstone, New York, NY, 1984:789-818

328. Boldt J, Knothe C, Welters I, Dapper FL, Hempelmann G. Normothermic versus hypothermic cardiopulmonary bypass: do changes in coagulation differ? Ann Thor Surg. 1996; 62:130-5

329. Mallet ML. Pathophysiology of accidental hypothermia. QJ Med 2002; 95:775785

330. Wong KC. Physiology and pharmacology of hypothermia. West J Med 1983;

138: $227-232$

331. Roscher R, Arlock P, Sjoberg T, Steen S. Effects of dopamine on porcine myocardial action potentials and contraction at 37 degrees $\mathrm{C}$ and 32 degrees $\mathrm{C}$. Acta Anaesthesiol Scand 2001;45:421-6

332. Morris DL, Chambers HF, Morris MG, Sande MA. Haemodynamic characteristics of patients with hypothermia due to occult infection and other causes. Ann Intern Med 1985; 102:153 - 7

333. Reuler JB, Parker R. Peritoneal dialysis in the management of hypothermia. JAMA 1978; 240: 2289-90

334. Vasallo SU, Delaney KA, Hoffman RS, Slater W, Goldfrank LR. A prospective evaluation of the electrographic manifestations of hypothermia. Acad Emerg Med 1999; 6: 1121-6

335. Kiyosue T, Arita M, Muramatsu H, Spindler AJ, Noble D. Ionic mechanisms of action potential prolongation at low temperature in guinea-pig ventricular 
monocytes. J Physiol 1993; 468:85 -106

336. Solomon A, Barish RA, Browne B, Tso E. The electrocardiographic features of hypothermia. J Emerg Med 1989; 7: 169-73

337. Bashow TT, Gualoberto A, Ryan C. Atrioventricular block in accidental hypothermia: a case report. Angiology 1989; 40:63-6

338. Talbot JH. The physiological and therapeutic effects of hypothermia. $N E n g J$ Med $1941 ; 224: 281-8$

339. Battin MR, Penrice J, Gunn TR, Gunn AJ. Treatment of term infants with head cooling and mild systemic hypothermia ( 35 degrees $\mathrm{C}$ and 34.5 degrees $\mathrm{C}$ ) after perinatal asphyxia. Pediatr 2003; 111:224-251

340. Shankaran S, Laptook A, Wright LL, Ehrenkranz RA, Donovan EF, Fanaroff AA et al. Whole body hypothermia for neonatal encephalopathy : animal observations as a basis for a randomised controlled pilot study in term infants.

Pediatr 2002; 110: $377-385$

341. Dobson GP. Organ arrest, protection and preservation: natural hibernation to cardiac surgery. Comp biochem physiol B biochem mol biol 2004;139: 469-85 342. Zheng J, Ishibashi Y, Zhang Y, Eigelshoven MH, Dunker DJ, Merkle H, Bache RJ, Uqurbil K, From AH. Myocardial biogenetics during acute hibernation. Am J Physiol 1997; 273: H1452-63

343. Gilbert M, Busund R, Skagseth A, Nilsen PA, Solbo JP. Resuscitation from accidental hypothermia of $13.7^{\circ} \mathrm{C}$ with circulatory arrest.

Lancet 2000;355:375-76

344. Azzopardi D, Robertson NJ, Cowan FM, Rutherford MA, Rampling M, Edwards AD. Pilot study of treatment with whole body hypothermia for neonatal encephalopathy. Pediatr. 2000; 106(4): 684-694. 
345. Currie AE, Kotecha S. Chronic lung disease of prematurely. Crit Care Ill. $1997 ; 12: 14-17$

346. Kotecha S. Cytokines in chronic lung disease of prematurity. Eur J Paediatr. $1996 ; 155: 514-517$

347. Fortenberry JD, Bhardwaj V, Niemer P, Cornish JD, Wright JA, Bland L. Neutrophil and cytokine activation with neonatal ECMO.

J Pediatr;1996;128:670- 678

348. Plotz FB, van Oeveren W, Bartlett RH, Wildevuur CRH. Blood activation during neonatal extracorporeal life support. J Thorac Cardiovasc Surg 1993;105:823-32 349. Menasche P, Peynet J, Haeffiner-Cavaillon N, Carreno MP, de Chaumaray T, Dillisse $\mathrm{V}$ et al. Influence of temperature on neutrophil trafficking during clinical cardiopulmonary bypass. Circulation 1995; 92:II-334-340.

350. Graziani L.J, Streletz L.J, Baumgart S, Cullen J, McKee L.M. Predictive value of neonatal electroencephalograms before and during extracorporeal membrane oxygenation. J Pediatr 1994;125:969-75

351. Naqeeb N, Edwards A.D, Cowan F.M, Azzopardi D. Assessment of neonatal encephalopathy by amplitude-integrated electroencephalography.

Pediatrics 1999; 103: 1263-1271

352. Hellstrom Westas L. Comparison between tape-recorded and amplitude integrated EEG monitoring in sick newborn neonates.

Acta Paediatr 1992;81:812-819

353. Griesen G. Tape recorded EEG and the cerebral function monitor: amplitude integrated, time compressed EEG. J Perinat Med 1994; 22: 541-546

354. The Hypothermia after Cardiac Arrest Study Group. Mild therapeutic hypothermia to improve the neurological outcome after cardiac arrest. 
N Engl J Med.2002;346:549-56

355. Stecker MM, Cheung AT, Pochettino A, Kent GP, Patterson T, Weiss, Bavaria JE. Deep hypothermia circulatory arrest I. Effects of cooling on electroencephalogram and evoked potentials. Ann Thorac Surg 2001;71:14-21 356. Reilly EL, Brunberg JA, Doty DB. The effect of deep hypothermia and total circulatory arrest on the electroencephalogram in children. Electroencephalogr Clin Neurophysiol 1974;36:611-7

357. De Sanctis JT, Bramson RT, Blickman JG. Can clinical parameters help reliably predict the onset of acute intracranial haemorrhage in infants receiving extracorporeal membrane oxygenation? Radiology 1996; 199: 429-52

358. Taylor GA, Fitz CR, Miller MK et al. Intracranial abnormalities in infants treated with extracorporeal membrane oxygenation: Imaging with US and CT. Radiology $1987 ; 165: 675-678$.

359. Lazar E.L, Abramson SJ, Weinstein S, Stolar C.J.H. Neuroimaging of brain injury in neonates treated with extracorporeal membrane oxygenation: lessons learned from serial examinations. J Pediatr Surg 1994:29;186 -191 